\title{
NUCLEAR GROUND STATE CHARGE RADII FROM ELECTROMAGNETIC INTERACTIONS
}

\author{
G. FRICKE and C. BERNHARDT \\ Institut für Kernphysik, Universität Mainz \\ J.-J.-Becher-Weg 45, D-55099 Mainz, Germany \\ K. HEILIG \\ Institut für Atom- und Molekülphysik, Universität Hannover \\ Appelstrasse 2, D-30167 Hannover, Germany \\ L. A. SCHALLER and L. SCHELLENBERG \\ Institut de Physique, Université de Fribourg \\ Pérolles, $\mathrm{CH}-1700$ Fribourg, Switzerland \\ E. B. SHERA \\ Los Alamos National Laboratory \\ Los Alamos, New Mexico 87545 \\ and \\ C. W. DE JAGER \\ National Institute for Nuclear Physics and High Energy Physics \\ P.O. Box 41882, 1009 DB Amsterdam, The Netherlands
}

The Tables summarize experimental results from muonic atom transition energies, nuclear charge parameters from elastic electron scattering, and $K \mathrm{x}$-ray isotope shifts in so far as they provide information on nuclear ground-state charge radii. Numerous experimental results for optical isotope shifts have been published elsewhere; for eight elements the relevant information is condensed ("projected") here to one optical line per element. A model-independent analysis which combines data from all three experimental methods is applied to these elements and is presented as an illustration of the improved accuracy for the rms radii and Barrett radii which result from this analysis. (1) 1995 Academic Press, Inc. 


\section{CONTENTS}

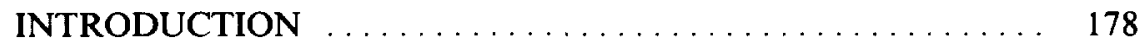

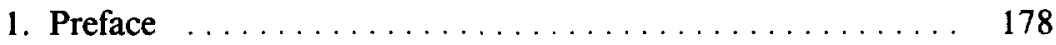

2. Optical Spectroscopy ...................... 179

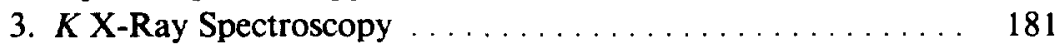

4. Muonic Atom Spectroscopy ................ 181

5. Elastic Electron Scattering Measurements f. . . . . . . . 184

6. Combined Analysis $\ldots \ldots \ldots \ldots \ldots \ldots \ldots \ldots$

7. Appendix A: King Plot ................... 190

8. Appendix B: Sign Conventions ................ 191

References for the Introduction $\ldots \ldots \ldots \ldots \ldots 2$

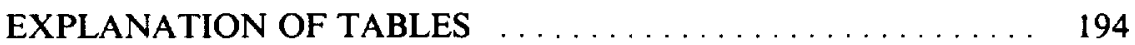

TABLES

I. Observed (Projected) Optical Isotope Shifts of Selected Elements ....................... 201

II. Observed $K \alpha \mathrm{X}$-Ray Isotope Shifts $\delta E$ and Charge Radius Variations $\lambda \ldots \ldots \ldots \ldots \ldots \ldots \ldots \ldots \ldots . \ldots \ldots$

IIIA. Muonic $2 p \rightarrow 1 s$ Transition Energies and Barrett Radii for $Z<60$ and $Z>77 \ldots \ldots \ldots \ldots \ldots \ldots \ldots$

IIIB. Muonic $2 p \rightarrow 1 s$ Transition Energies and Relative Intensities of Deformed Nuclei with $60 \leqslant Z \leqslant 77 \ldots \ldots \ldots 228$

IIIC. Barrett Radii and Related Parameters of Deformed Nuclei with $60 \leqslant Z \leqslant 77 \ldots \ldots \ldots \ldots \ldots \ldots \ldots \ldots .237$

IV. Confit Matrices for Muonic $2 p \rightarrow 1 s$ Energy Differences of Isotopes, $31 \leqslant Z \leqslant 58 \ldots \ldots \ldots \ldots \ldots \ldots \ldots \ldots 243$

V. Differences of Barrett Radii for Isotopes, $6 \leqslant Z \leqslant 82 \ldots 251$

VI. Confit Matrices for Muonic $2 p \rightarrow 1 s$ Energy Differences of Isotones, $10 \leqslant N \leqslant 82 \ldots \ldots \ldots \ldots \ldots \ldots \ldots \ldots$

VII. Differences of Barrett Radii for Isotones, $8 \leqslant N \leqslant 126 \ldots 260$

VIII. Charge Density Distribution Parameters from Elastic Electron Scattering $\ldots \ldots \ldots \ldots \ldots \ldots \ldots \ldots 26 \ldots \ldots \ldots$

IX. Fourier-Bessel Coefficients from Elastic Electron Scattering 264

X. King Plots: Optical versus Combined Muonic and Elastic Electron Scattering Data ............... 268

XI. Electronic Factor $F$ and Specific to Normal Mass Shift Ratio SMS/NMS for Projected Optical Lines . . . . . . . 277

XII. Root-Mean-Square Charge Radii from the Combined Analysis of Optical, Muonic, and Elastic Electron Scattering Data ........................ 278

REFERENCES FOR TABLES $\ldots \ldots \ldots \ldots \ldots \ldots \ldots \ldots . \ldots \ldots$

\section{INTRODUCTION}

\section{Preface}

This compilation contains nuclear ground-state charge radii from measurements probing the nucleus with the well-understood electromagnetic interaction. Methods using strong forces to determine nuclear radii are not included. Since the previous compilations of results from 
four electromagnetic methods, namely measurements of optical transitions ${ }^{1}$ and $K \mathrm{x}$ rays, ${ }^{2}$ determination of transition energies in muonic atoms, ${ }^{3}$ and elastic electron scattering experiments, ${ }^{4}$ a vast amount of new experimental information on the electromagnetic structure of the nuclear ground state of many isotopes has become available, and the accuracies have been improved. So it seems useful to give an up-to-date summary.

Muonic atom and electron scattering investigations require at least tens of milligrams of target material. So far only the optical method is capable of measuring unstable isotopes when only tiny amounts of material are available. Modern laser-spectroscopic techniques for optical isotope shift measurements have made it possible to reach even short-lived (down to $1 \mathrm{~s}$ ) unstable isotopes. ${ }^{5}$ Hence, many experimental and theoretical optical isotope shift investigations are still in progress. ${ }^{6}$ The main goal of most of the publications today is no longer the determination of radii differences but the search for more subtle effects in the electron shells of the atom. So the time has come to make a resumé of our present knowledge on nuclear charge sizes. For this purpose, the input from muonic atom and electron scattering data is vital.

The muon factories at Los Alamos (LAMPF) and at Villigen (PSI, formerly SIN) started their operation in 1974. They delivered orders of magnitude higher muonbeam fluxes than before achieved elsewhere, yielding results for muonic $\mathrm{x}$-ray transition measurements with higher precision. Therefore, systematic effects of isotope and isotone shifts could be determined. Up to now isotone shifts of high accuracy could be deduced only from muonic atoms. The precision of the nuclear charge radii deduced from muonic atoms is in most cases limited by the error in the calculation of the nuclear polarization corrections rather than by counting statistics or other uncertainties. Almost all stable isotopes have now been measured by the muonic $\mathrm{x}$-ray transition technique. This provides another reason for a new nuclear ground-state charge radii compilation.

The four electromagnetic methods are sensitive to different properties of the nuclear ground-state charge distributions. The optical transitions and the $K \mathrm{x}$ rays are sensitive to the differences of the mean-square charge radii $\delta\left\langle r^{2}\right\rangle$ between isotopes, with small contributions from higher radial moments $\delta\left\langle r^{4}\right\rangle$ and $\delta\left\langle r^{6}\right\rangle$. The radial moments are defined by

$$
\langle K(r)\rangle=\frac{1}{\mathrm{Ze}} \int \rho_{\mathrm{N}}(r) K(r) d \tau .
$$

Specifically,

$$
\left\langle r^{2}\right\rangle=\frac{1}{\mathrm{Ze}} \int \rho_{\mathrm{N}}(r) r^{2} d \tau
$$

and

$$
\left\langle r^{n}\right\rangle=\frac{1}{\mathrm{Ze}} \int \rho_{\mathrm{N}}(r) r^{n} d \tau
$$

where $\rho_{\mathrm{N}}(r)$ is the nuclear charge density distribution and $\int \rho_{\mathrm{N}}(r) d \tau=\mathrm{Ze}$.

The transition energies in muonic atoms are sensitive to the Barrett equivalent radius ${ }^{7} R_{k \alpha}$, with

$$
K(r)=r^{k} e^{-\alpha r} \text {. }
$$

(Throughout this paper $R_{k \alpha}$ always denotes the Barrett equivalent radius.) On the other hand, the charge distribution $\rho_{\mathrm{N}}(r)$ can be deduced from elastic electron scattering cross sections. A combination of the data from different experimental methods generally yields more detailed and accurate knowledge of the nuclear radii than is available from any single method. Because of the different quantities determined by each method, one has to be careful that no model-dependent bias is introduced when the data are combined. However, in the following it will be shown that with the help of a combined analysis of elastic electron scattering data, muonic transition energies, and optical and $K \mathrm{x}$-ray measurements, nearly modelindependent values for the root-mean-square (rms) charge radii of stable nuclei and at least the $\lambda$ values (see $\mathrm{Eq}$. (2.7)) for the chains of unstable isotopes can be deduced with high precision.

In addition, two important factors for optical transitions between different electronic states can be evaluated by such a combined analysis, namely the change of the electron density at the nucleus, represented by $F_{i}$, and the mass shift, described by the factor $M_{i}$ (see Section 2). The factors $F_{i}$ and $M_{i}$ can thus be determined from experimental data, making it possible to derive $\delta\left\langle r^{2}\right\rangle$ without any knowledge of the electron shell properties. Moreover, the factor $F_{i}$ is a valuable experimental input for the testing of calculations of electronic wave functions.

In the next four sections a short survey of the four different electromagnetic techniques for the determination of nuclear charge radii is given. The information contained in the measured quantities is discussed and the particular advantages of each method are emphasized. The general ideas and the procedure of a combined model-independent analysis and the results are discussed in the concluding Section 6.

\section{Optical Spectroscopy}

Outer atomic electrons may serve as a probe of the nuclear structure. The isotope shift (IS) of optical transitions is observed as a small energy shift of the centers of gravity of the spectral components of different isotopes in optical transitions. It is well known ${ }^{1,8,9}$ that the IS has two causes. These are distinct isotope-dependent nuclear properties that interact with the electrons of the shells, the nonzero nuclear size and the finite nuclear mass, giving rise, respectively, to the field (or volume) shift (FS) and the mass shift (MS). The observed shift $\delta \nu_{i}^{A A^{\prime}}$ between two 
isotopes with mass numbers $A$ and $A^{\prime}$ in an atomic spectral line $i$ of wavenumber or frequency $\nu$ is the sum of FS and MS; i.e.,

$$
\delta \nu_{i}^{A A^{\prime}}=\delta \nu_{i, \mathrm{FS}}^{A A^{\prime}}+\delta \nu_{i, \mathrm{MS}}^{A A^{\prime}} .
$$

It is customary to split the observed mass shift into two contributions, the trivial normal mass shift (NMS; reduced mass) and the very difficult to calculate specific mass shift (SMS; correlations between electrons):

$$
\delta \nu_{i, \mathrm{MS}}^{A A^{\prime}}=\delta \nu_{i, \mathrm{NMS}}^{A A^{\prime}}+\delta \nu_{i, \mathrm{SMS}}^{A A^{\prime}} .
$$

These shifts depend in the same way on the atomic masses $A$ and $A^{\prime}$,

$$
\begin{aligned}
\delta \nu_{i, \mathrm{MS}}^{A A^{\prime}} & =M_{i} \frac{A-A^{\prime}}{A A^{\prime}}, \\
\delta \nu_{i, \mathrm{NMS}}^{A A^{\prime}} & =\frac{\nu_{i}}{1822.9} \frac{A-A^{\prime}}{A A^{\prime}},
\end{aligned}
$$

where the factor $M_{i}$ is the mass shift factor containing contributions of NMS and SMS. ${ }^{8,9}$ The NMS always shifts the heavier isotopes toward larger energies (or wavenumbers). By convention this is called a positive IS in the line. Thus, the MS decreases with increasing $A$, and the relative MSs for isotopes $A, A^{*}=A+2$, and $A^{* *}=A+4$ are related through

$$
\delta \nu_{i, \mathrm{MS}}^{A A^{*}}: \delta \nu_{i, \mathrm{MS}}^{A^{*} A^{* *}}=A^{* *}: A .
$$

Good theoretical calculations of the SMS exist only for a few elements, for example, for $\mathrm{Ca}^{10}$ In all other cases rather rough estimates (for example, by comparison with results in light elements) have to be used for the SMS,${ }^{8}$ if no information from other than optical investigations is available.

The FS reflects the isotopic variation of the meansquare nuclear charge radius $\delta\left\langle r^{2}\right\rangle$. Traditionally the FS in a transition $i$ is written as

$$
\delta \nu_{i, \mathrm{FS}}^{A A^{\prime}}=F_{i} \lambda^{A A^{\prime}},
$$

where

$$
\begin{aligned}
& \lambda^{A A^{\prime}}=\delta\left\langle r^{2}\right\rangle^{A A^{\prime}}+\left(C_{2} / C_{1}\right) \delta\left\langle r^{4}\right\rangle^{A A^{\prime}} \\
& +\left(C_{3} / C_{1}\right) \delta\left\langle r^{6}\right\rangle^{A A^{\prime}}+\cdots,
\end{aligned}
$$

with the convention that $\delta\left\langle r^{2}\right\rangle^{A A^{\prime}}=\left\langle r^{2}\right\rangle^{A}-\left\langle r^{2}\right\rangle^{A^{\prime}}$. This parameterization was introduced in 1965 by Seltzer; the $C$ coefficients were tabulated in Refs. 2 and 11 , and corrections were added in Ref. 12 (for further explanations see also Refs. 1, 9, and 13). $F_{i}$ is the electronic factor, which is proportional to the change of the electronic charge density at the nucleus in the transition $i$ under investigation. The FS percentage is largest in transitions that involve $s$ electrons. In order to determine $\lambda^{A A^{\prime}}$ from the FS, the electronic factor $F_{i}$ has to be known. Different procedures have been used so far for its determination, as discussed below. (i) Semiempirical methods (Goudsmit-FermiSegrè; see, for example, Refs. 1, 8, and 9). For alkalilike $n s-n p$ transitions the following expressions hold:

$$
\begin{array}{r}
F_{i}=E_{i} f(Z), \quad E_{i}=\beta E_{n s}, \quad E_{n s}=\pi a_{0}^{3}|\psi(0)|_{n s}^{2} / Z, \\
\beta=\frac{\Delta|\psi(0)|_{n s-n p}^{2}}{|\psi(0)|_{n s}^{2}} .
\end{array}
$$

In these expressions, $f(Z)$ is the correction factor for the finite size of the nucleus and for relativistic effects, ${ }^{14-16} \beta$ is a screening factor caused by the screening of lower, closed shells, ${ }^{1} a_{0}$ is the Bohr radius, and $\Delta|\psi(0)|_{n s-n p}^{2}$ is the nonrelativistic change of the electron charge density in an $n s-n p$ transition at the nucleus. The probability density $|\psi(0)|_{n s}^{2}$ is calculated either from the fine structure ( $n s$ series) or from the hyperfine structure ( $a_{s}$ factor). Similar procedures are in principle possible with other kinds of transitions. ${ }^{\prime}$

(ii) $A b$ initio calculations made with self-consistentfield procedures. For many elements calculations with a multiconfigurational Dirac-Fock method have been done or are in progress. ${ }^{17,18}$

(iii) A rather new procedure utilized in the present paper, wherein only measured values enter into the determination of $F_{i}$ and $M_{i}$. No information is needed about $\Delta|\psi(0)|^{2}$, the purity of configurations, or mass shifts. The procedure makes use of a special King plot, in which the so-called scaled IS (see Appendix A) of a selected optimal line is plotted versus the scaled $\lambda$ values for the same isotope pairs. The $\lambda$ values are evaluated in a combined analysis of muonic atoms and elastic electron scattering data (see Section 6).

From Eq. (2.3) it follows that the relative mass shifts for a chain of isotopes are exactly the same in all optical lines. The relative field shifts-differing from the mass shift ratios-are also the same in all lines; see Eq. (2.6). The ratio of mass shift to field shift varies-often strongly - from line to line. These correlations are used to connect the measurements in different lines.

To explain the procedure, let us assume as a very simple case that three isotope pairs have been investigated in two optical lines. The evaluation will be done by a (two-dimensional) King plot (see Appendix A). The proper projection onto the regression line in this plot gives improved IS values for each of both lines. Naturally, it would be even more reliable to use many more lines for this procedure. The result is a so-called multidimensional King plot $^{19}$ (variance-covariance matrix). Any of the lines in that plot may in principle be taken as the line to be projected. For the projection, the desired characteristic in the selected line is that the observed relative total ISs differ as much as possible from the relative MSs (Eq. (2.3)) and that the accuracy of the measurements is adequate. 
Electrons with nonzero orbital angular momentum have a very small or negligible charge density at the nucleus. Nevertheless, due to screening factors similar to $\beta$, large FSs may also occur in some non $s-p$ transitions. Furthermore, in alkali-like electronic transitions the $s_{1 / 2}-p_{1 / 2} \mathrm{FS}$ is often nearly the same as the $s_{1 / 2}-p_{3 / 2} \mathrm{FS}$. The MS happens to be also the same for both $p_{1 / 2}$ and $p_{3 / 2}$ electrons. ${ }^{20}$

A number of elements were selected to illustrate the procedure of the combined analysis (Section 6). The corrected shifts for the projected lines of these elements are given in Table I. They are used together with the combined results from elastic electron scattering and muonic $\mathrm{x}$ rays to evaluate the optical factors $F_{i}$ and $M_{i}$ by a special King plot. By means of ordinary (two dimensional) King plots, the $F_{i}$ and $M_{i}$ values for all other investigated optical lines can then be determined easily. This will be explained in detail in the next sections. For optical IS measurements no nuclear polarization corrections ${ }^{21}$ have been applied. Why this might be permitted is explained in Appendix A.

\section{3. $K$ X-Ray Spectroscopy}

The isotope shift of $K \mathrm{x}$-ray transitions has the same two origins as the IS in optical transitions: field effect and mass effect. In contrast to optical shift effects from outer electrons, the calculation of the electronic factors $F_{i}$ and $M_{i}$ is much easier and more reliable for the inner-shell xray transitions (see Refs. 2 and 22).

The difficulties of $x$-ray isotope shift measurements lie in experimental restrictions: no $x$-ray interferometers and no tunable, narrowband $x$-ray lasers exist hitherto. The measurements thus have had to be performed with curved crystal spectrometers. Therefore, the relative experimental errors range from a few to about $50 \%$. In modern optical laser-spectroscopic measurements, on the other hand, the errors are in most cases smaller by at least one order of magnitude.

The nuclear parameter $\lambda^{A A^{\prime}}$ (Eq. (2.7)) can be determined analogously as in optical spectroscopy: Eqs. (2.1) to (2.7) also hold for $K \mathrm{x}$-ray isotope shifts. Most of the experimental results date back to before 1974. They are listed in Ref. 2 and are included in Table II together with the only measurement since 1974 , the shift of the $\mathrm{Pb}$ isotopes. ${ }^{23}$ This new measurement together with the data from Lee and Boehm ${ }^{24}$ yields absolute and accurate values for the radii differences of the $\mathrm{Pb}$ isotopes, which can be compared with the combined results from muonic $\mathrm{x}$ rays and elastic electron scattering (see Fig. 1). Here the $\lambda^{A A^{\prime}}$ values derived from the three experimental methods are plotted; a diagonal line at $45^{\circ}$ is added for convenience. The excellent agreement of these results shows the reliability for each of the three independent methods and suggests the reliability of the combined analysis of optical,

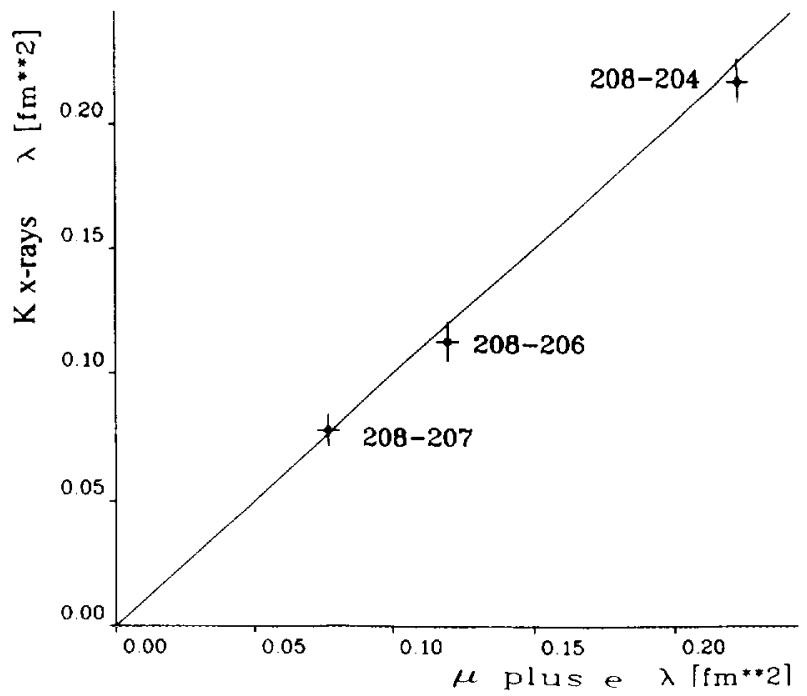

Figure 1. Values of $\lambda^{A A^{\prime}}$ (in $\mathrm{fm}^{2}$ ) from $K \mathrm{x}$ rays versus those from the combined results of muonic $x$-ray spectroscopy and elastic electron scattering for the stable $\mathrm{Pb}$ isotopes. Numbers beside the data points refer to isotope pairs. The $45^{\circ}$ line is added for convenience.

muonic $\mathrm{x}$-ray, and electron scattering data (see Section 6) for absolute calibration of optical isotope shift measurements from experimental data only, without model assumptions. Details will be given in the next sections.

\section{Muonic Atom Spectroscopy}

A compilation entitled "Charge-Distribution $\mathrm{Pa}$ rameters, Isotope Shifts, Isomer Shifts, and Magnetic $\mathrm{Hy}$ perfine Constants from Muonic Atoms," by Engfer et al., ${ }^{3}$ appeared in 1974 in ATOMIC DATA AND NUCLEAR DATA TABLES. Subsequent to that date the muon factories LAMPF and PSI came into operation and delivered muon beams with much higher intensities. As a result, a wealth of new and more precise data has been obtained. The investigation of almost all stable isotopes became possible. From the muonic $2 p-1 s$ transition energies given in Ref. 25 , nuclear charge radii are deduced in the present work. The accuracy of almost all but the lightest nuclear radii is limited no longer by the experimental error but by the uncertainty of the calculated nuclear polarization correction.

The procedure for deducing a nuclear charge radius from the measured transition energies has been described in detail in the above referenced summary. ${ }^{3}$ Hence, only a short description for such an analysis is given in the following. 
The transition energies in muonic atoms are strongly affected by the size of the nucleus. In first-order perturbation theory the energy shift of a muonic transition between levels $i$ and $f$ due to this "finite size" effect is given by ${ }^{3}$

$$
\delta E_{i f}=4 \pi \int_{0}^{\infty} \delta \rho_{\mathrm{N}}(r)\left[V_{\mu}^{i}(r)-V_{\mu}^{f}(r)\right] r^{2} d r .
$$

Here $V_{\mu}^{i}(r)$ and $V_{\mu}^{f}(r)$ are the potentials generated by the bound muon in the states $i$ and $f$, respectively, and $\delta \rho_{N}(r)$ denotes a variation of the spherical charge distribution of the nucleus.

In the region where $\rho_{\mathrm{N}}(r) r^{2}$ is large, the difference $V_{\mu}^{i}-V_{\mu}^{f}$ can be approximated well by the analytic expression $B r^{k} e^{-\alpha r}$, as has been suggested by Barrett. ${ }^{7}$ Thus, perturbation theory implies that a Barrett moment

$$
\left\langle r^{k} e^{-\alpha r}\right\rangle=\frac{4 \pi}{\mathrm{Ze}} \int_{0}^{\infty} \rho_{\mathrm{N}}(r) r^{k} e^{-\alpha r} r^{2} d r
$$

may be deduced model-independently from the experimental transition energy $E_{i j}$. With this moment an equivalent radius $R_{k \alpha}$, which has a dimension of a length, can be defined by the implicit equation

$$
3\left[R_{k \alpha}\right]^{-3} \int_{0}^{R_{k \alpha}} r^{k} e^{-\alpha r} r^{2} d r=\left\langle r^{k} e^{-\alpha r}\right\rangle
$$

$R_{k \alpha}$ is thus the radius of a sphere with constant charge density which yields the same moment $\left\langle r^{k} e^{-\alpha r}\right\rangle$ as the actual nuclear charge distribution. Throughout this compilation, $R_{k \alpha}$ always denotes the Barrett equivalent radius and $R_{k \alpha}^{\mu}$ the one deduced from the muonic atom $2 p-1 s$ transitions.

For an exact analysis, the eigenvalues of the Dirac equation with an analytic parametrization of $\rho_{\mathrm{N}}(r)$ are determined numerically by fitting the free parameters of the charge distribution used to the experimental transition energies. As usual, for spherical nuclei the two-parameter Fermi charge distribution (Fermi II) was used,

$$
\rho_{\mathrm{N}}(r)=\rho_{0}(1+\exp [(r-c) / a])^{-1},
$$

where $c$ is the half-density radius. The surface thickness $t$ of the distribution is

$$
t=4 a \ln 3=4.394 a .
$$

For deformed nuclei the half-density radius is written as

$$
c=R_{0}\left[1+\beta_{2} Y_{20}(\theta, \phi)\right] .
$$

Here, $\beta_{2} Y_{20}$ gives the angular variation of $c, \beta_{2}$ is the quadrupole deformation parameter, and $R_{0}$ is the monopole radius. From the fitted charge distributions, a meansquare charge radius is calculated using Eq. (1.2).

In the next step the parameters $B, k$, and $\alpha$ of the Barrett moment were fitted to reproduce the muon po- tential difference $V_{\mu}^{i}(r)-V_{\mu}^{f}(r)$ (see Eq. (4.1)). To optimize the model independence, this was done separately for every transition to every numerically calculated muon potential difference. Then the Barrett moments $\left\langle r^{k} e^{-\alpha r}\right\rangle$ and the corresponding equivalent radii $R_{k \alpha}^{\mu}$ were calculated from $\rho_{N}(r)$.

The degree of model independence of the $R_{k \alpha}^{\mu} \mathrm{com}$ pared to the rms radii is illustrated with ${ }^{40} \mathrm{Ca}$ and ${ }^{208} \mathrm{~Pb}$ in Fig. 2 using a two-parameter Fermi distribution for $\rho_{\mathrm{N}}(r)$. By adjusting the half-density radius $c$ to the measured transition energy with a given surface parameter, a change in the assumed surface thickness from $t=1.8 \mathrm{fm}$ to $t=2.8 \mathrm{fm}$ results in a change in the rms radius of 20 am $\left(1 \mathrm{am}=10^{-18} \mathrm{~m}\right)$ for ${ }^{40} \mathrm{Ca}$ and $50 \mathrm{am}$ for ${ }^{208} \mathrm{~Pb}$, whereas the corresponding change in the Barrett radius $R_{k \alpha}^{\mu}$ amounts to an impressively small value ${ }^{26}$ of just $0.2 \mathrm{am}$ for ${ }^{208} \mathrm{~Pb}$. This shows the model independence of $R_{k \alpha}^{\mu}$, since the nuclear polarization uncertainty or the experimental error is always larger than 0.2 am, as can easily be verified with the help of the factor $C_{z}$ (see Tables IIIA and IIIC): $C_{z}$ converts a small change in the transition energy into a small change of the $R_{k \alpha}^{\mu}$ radius.

Table IIIA presents results for $Z<60$ and $Z>77$, namely the experimental $2 p-1 s$ energies, the fitted energies, the nuclear polarization correction, the adjusted halfdensity radius $c$, the model-dependent rms radius, the Barrett parameters $\alpha$ and $k$, the factor $C_{z}$, and finally $R_{k \alpha}^{\mu}$. The error given for the $2 p-1 s$ energies is statistical

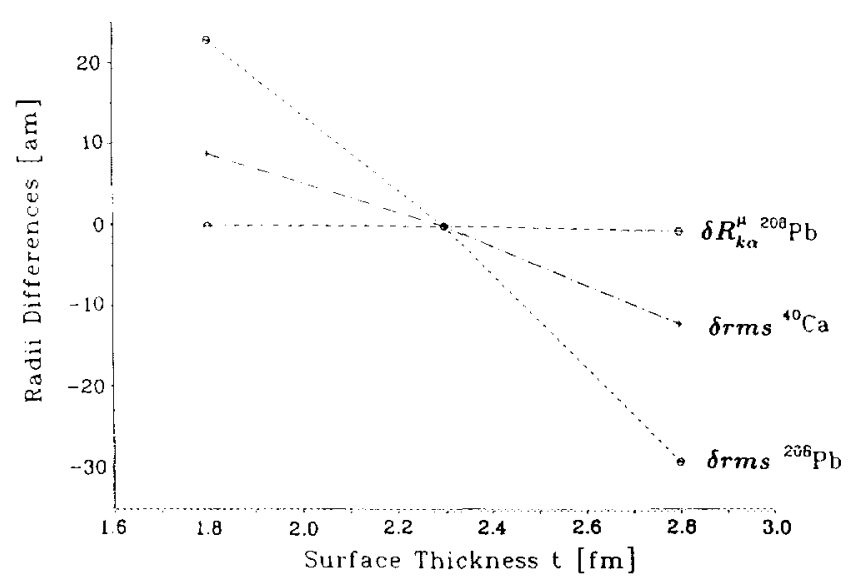

Figure 2. Illustration of the model independence of the Barrett radii $R_{k a}^{u}$ compared to the model-dependent $\mathrm{rms}$ radii. Plotted is $\delta R_{k_{\alpha}}^{u}(t)=R_{k_{\alpha}}^{\mu}(t=2.3 \mathrm{fm})-R_{k_{\alpha}}^{u}(t)$ for ${ }^{208} \mathrm{~Pb}$ and $\delta \mathrm{rms}(t)=\operatorname{rms}(t=$ $2.3 \mathrm{fm})-\operatorname{rms}(t)$ for ${ }^{40} \mathrm{Ca}$ and ${ }^{208} \mathrm{~Pb}$, where $t$ is the surface thickness of a two-parameter Fermi charge distribution (Eq. (4.4)). The variation of $R_{k \alpha}^{\mu}$ between $t=1.8 \mathrm{fm}$ and $t=2.8 \mathrm{fm}$ for $\mathrm{Pb}$ is less than $0.2 \mathrm{am}\left(1 \mathrm{am}=10^{-18} \mathrm{~m}\right)$, whereas the model-dependent $\mathrm{rms}$ radius decreases by $50 \mathrm{am}$. 
only and is computed using a weighted least-squares adjustment procedure which includes all elements from oxygen to samarium. ${ }^{25}$ The $\chi^{2}$ value per degree of freedom of this adjustment was 1.93. No systematic error like the nonlinearity in the electronics is included. The first error on the $R_{k \alpha}^{\mu}$ is obtained by multiplying the statistical error of the $2 p-1 s$ energies by the sensitivity factors $C_{z}=$ $d R_{k \alpha}^{\mu} / d E$. The second error is due to the uncertainty in the calculation of the nuclear polarization corrections. This error was conservatively estimated by Rinker $^{27}$ to be $30 \%$ of the total nuclear polarization value. The nuclear data for the calculation of the nuclear polarization corrections (excitation energies and $B(E 2)$ values) are taken from the latest Nuclear Data Sheets. The rms radii $\left\langle r^{2}\right\rangle_{\text {model }}^{1 / 2}$ (no error assigned) given in Tables IIIA and IIIC have the suffix "model" to point out that this value is based on the Fermi two-parameter charge distribution and is model-dependent as can be seen from Fig. 2. Nevertheless, a reasonable systematic error for the $\left\langle r^{2}\right\rangle_{\text {model }}^{1 / 2}$ values, which are calculated with the skin thickness $t=2.3 \mathrm{fm}$, may be obtained from this figure by varying the skin thickness parameter by $\pm 10 \%$. This is supported by a direct comparison of the rms values in Tables III and XII. Note that the relative statistical error of the $\left\langle r^{2}\right\rangle_{\text {model }}^{1 / 2}$ values is the same as that of the $R_{k \alpha}^{\mu}$ values from Tables III.

For deformed nuclei with $60 \leqslant Z \leqslant 77$ (see Table IIIB), in addition to the static quadrupole interaction, there is a strong dynamic $E 2$ interaction between nuclear and muonic levels also for even-even nuclei. ${ }^{3,28}$ For odd nuclei, the observed spectra can become rather complex. As an example, the observed splitting in the ${ }^{181} \mathrm{Ta} 2 p-1 s$ transition into more than 30 components $^{29}$ is shown in Fig. 3. The literature contains further fitted parameters like quadrupole deformation and so forth. All components could be fitted remarkably well with a $\chi^{2}$ per degree of freedom of 1.2. In Table IIIB, up to 10 (20) of the strongest components for the $2 p_{3 / 2}-1 s_{1 / 2}$ and $2 p_{1 / 2}-1 s_{1 / 2}$ complex are listed for even (odd) deformed nuclei. Table IIIC shows, besides the nuclear polarization values for the center of gravity of the hyperfine components, the model parameters of the deformed Fermi-type charge density distribution and the fitted Barrett parameters. More details are found in the references.

Table IV contains in matrix form the energy differences between isotopes for the two $2 p-1 s$ transitions together with the experimental errors. For the small energy shifts between neighboring isotopes $(\approx 20 \mathrm{keV})$ or isotones $(\approx 200 \mathrm{keV})$, no energy calibration error is expected; so, the experimental error is determined mainly by statistics and to a minor part by the subtraction of the background of the spectrum. The related differences for the Barrett radii $\Delta R_{k \alpha}^{\mu}$ are given in Table $V$. There, the second error is the nuclear polarization uncertainty. As an upper limit, this error was estimated to be $10 \%$ of the larger of the

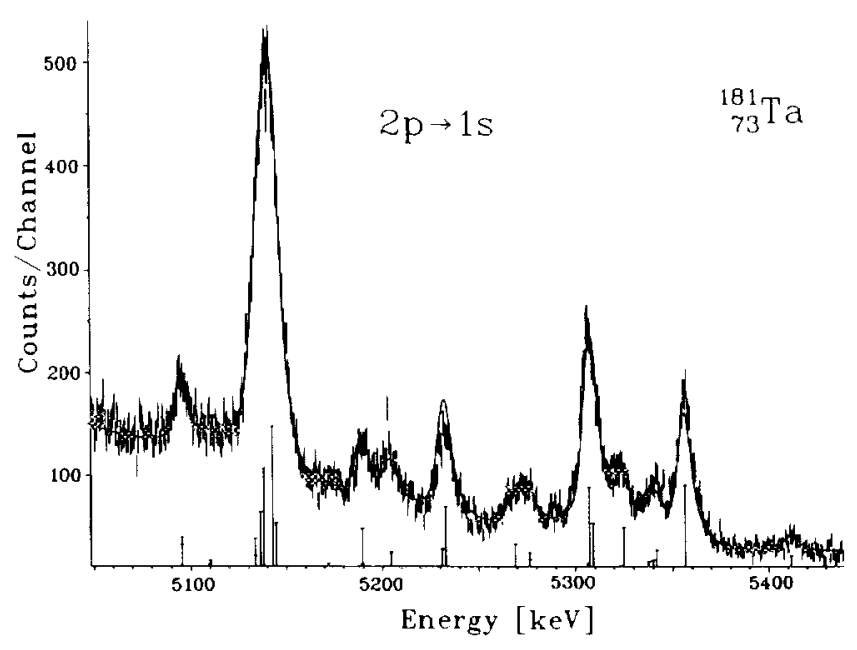

Figure 3. Energy spectrum in the region of the $2 p-1 s$ muonic atom transition complex for ${ }^{181} \mathrm{Ta}$ shown with the calculated components of the fitted curve (solid line). The fit gives $x^{2}=1.2$ per degree of freedom. The dynamic hyperfine interaction results in more than 30 components.

nuclear polarization values for the two isotopes. For deformed nuclei, $\frac{1}{3}$ of the larger error of the two isotopesas assigned to the absolute values of $R_{k \alpha}^{\mu}$-is given. Tables VI and VII give the corresponding data for isotones.

All $\Delta R_{k \alpha}^{\mu}$ values for even-even isotope pairs with $\Delta N=2$ are displayed in Figs. $4 \mathrm{a}-4 \mathrm{c}$. The $\Delta R_{k \alpha}^{\mu}$ values for even-even isotone pairs with $\Delta Z=2$ are shown in Figs. $5 \mathrm{a}$ and $5 \mathrm{~b}$. So far only muonic atoms can measure shifts accurately enough to show that the systematic behavior is similar for isotopes and isotones. There are strong shell effects: the differences in radii are largest at the beginning of a neutron or proton shell and decrease almost linearly from there. Toward the end of a shell the values may become constant (see, for example, Fig. $4 \mathrm{c}$ for isotopes and Fig. $5 \mathrm{~b}$ for isotones). Exceptions to the linear behavior are the $s-d$ shell and the europium region, where the behavior is modified by strong deformation changes of the nuclei. ${ }^{29,30}$ A more detailed discussion of the systematics of the radii differences can be found in Refs. 30-32. Since the Barrett parameters $k$ and $\alpha$ are fitted for each nucleus separately, different radial moments are compared. The model error introduced hereby has been evaluated by Bernhardt. ${ }^{29} \mathrm{It}$ is less than $0.2 \mathrm{am}$ for isotopes $(\Delta N=2)$ and less than $1.0 \mathrm{am}$ for isotones $(\Delta Z=2)$. For a detailed error discussion, see the relevant literature.

In spite of all these corrections, the total error in the Barrett radius $R_{k \alpha}^{u}$ deduced from muonic atoms is much smaller than the error of the same quantity calculated from the charge distribution resulting from an elec- 
tron scattering experiment. At present the finite size shift of muonic atom transitions is the most precise method for determining absolute nuclear radial moments.

\section{Elastic Electron Scattering Measurements}

In elastic electron scattering experiments the measured quantity is the differential cross section $d \sigma(E, \theta) /$ $d \Omega$ for the elastic scattering of an electron of energy $E$ through an angle $\theta$.

Although a careful analysis of the experimental cross sections must be performed with a phase shift code, ${ }^{33}$ the information contained in the cross section data can be discussed in the framework of the first Born approximation. ${ }^{34}$ In the case of a nucleus with spin $I=0$, where only spherical charge scattering contributes to the elastic cross section, $d \sigma / d \Omega$ can be factorized into $(d \sigma / d \Omega)_{\text {Mot }}$ for a point-like nucleus and a structure function, the form factor $F(q)$, which depends only on the momentum transfer $q=(2 E / \hbar c) \cdot \sin (\theta / 2)$, under the condition $m_{\mathrm{e}} c^{2} \ll E$ :

$$
\begin{array}{r}
\left.\frac{d \sigma}{d \Omega}\right|_{\text {exp }}=\left.\frac{d \sigma}{d \Omega}\right|_{\text {Mott }}|F(q)|^{2}, \\
\text { with }\left.\frac{d \sigma}{d \Omega}\right|_{\text {Mott }}=\frac{Z^{2} e^{4}}{4 E^{2}} \frac{\cos ^{2} \theta / 2}{\sin ^{4} \theta / 2} .
\end{array}
$$

The form factor $F(q)$ is related to the nuclear charge distribution $\rho_{\mathrm{N}}(r)$ through a Fourier-Bessel transformation:

$$
F(q)=\int \rho_{\mathrm{N}}(r) j_{0}(q r) d \tau, \quad 0<q<\infty
$$

The model-independent Fourier-Bessel analysis, introduced by Dreher et al., ${ }^{35}$ uses an expansion of the nuclear charge density distribution with the limitation $\rho_{\mathrm{N}}(r)=0$ for $r>R_{\text {cut }}$,

$$
\rho_{\mathrm{N}}(r)=\sum_{\nu=1}^{\infty} a_{\nu} j_{0}\left(q_{v} r\right)
$$

where $j_{0}(q r)=(\sin q r) /(q r)$ denotes the spherical Bessel function of order zero. The coefficients $a_{\nu}$ for $\nu=1 \cdots N$ are related directly to the form factor value at $q_{\nu}=\nu \pi /$ $R_{\text {cut }}$ by

$$
a_{\nu}=\frac{q_{\nu}^{2}}{2 \pi R_{\text {cut }}} F_{\text {exp }}\left(q_{\nu}\right)
$$

with $R_{\text {cut }}$ being the cutoff radius. The maximum value of the momentum transfer $q_{\max }$ is given by the experimental conditions. It determines the number of measured
Fourier-Bessel coefficients

$$
N=\frac{R_{\mathrm{cut}} q_{\mathrm{max}}}{\pi} .
$$

Beyond the cutoff radius, the nuclear charge distribution $\rho_{N}(r)$ is assumed to be zero. The behavior of the form factor $F(q)$ in the region $q>q_{\max }$ where no data were measured is assumed to be bounded by a $q^{-4} \exp \left(-q^{2}\left\langle r^{2}\right\rangle_{\mathrm{p}}\right)$ dependence, where $\left\langle r^{2}\right\rangle_{\mathrm{p}}^{1 / 2}$ is the rms radius of the proton. These assumptions originate from expectations for the distribution of the nucleons inside the nucleus and for the finite size of the nucleons, respectively. They yield an upper limit for the contributions from the higher Fourier-Bessel components $a_{\nu}$ with $\nu>$ $N$, which are not determined directly. This method ${ }^{35}$ has the advantage that uncertainties in the charge distribution originating from the experimental errors and from the lack of knowledge about the form factor for $q>q_{\max }$ can be determined separately. For the normalization we have adopted the convention that the integral over the nuclear charge density distribution equals the nuclear charge $\mathrm{Ze}$. By using the Fourier-Bessel expansion technique, realistic charge distribution error bands can be derived which reflect both the statistical error and the error due to the limited range of the experimentally accessible momentum transfer. ${ }^{35}$

Another parametrization, which is often used and was first introduced by Sick, ${ }^{36}$ is the "sum of Gaussians." More about this procedure can be found in the original literature or in the compilation by de Vries et al. ${ }^{4}$

Tables VIII and IX show elastic electron scattering results published in the time period from that last compilation $^{4}$ up to 1993 . The same notation as in the 1987 compilation is used. In Table VIII we present charge density distribution parameters and in Table IX the Fourier-Bessel coefficients $a_{\nu}$ according to Eq. (5.4) and the value of the cutoff radius used. The coefficients' errors are not given in the Table. Because these errors are strongly correlated, the uncertainties in the nuclear charge density distribution can be determined only from the full errorcorrelation matrices, which are not published in the literature. In the following the Fourier-Bessel coefficients are used without errors. A justification will be given in Section 6 and illustrated with the example of samarium. In addition, the values for $\left\langle r^{n}\right\rangle_{e}^{1 / n}, n=2,4,6$, and $R_{k \alpha}^{e}$ are given. This allows the computation of $V_{n}^{e}$ factors, with $n=2,4,6$, which will be used in the combined analysis in Section 6.

Figure 4. Isotope shifts of Barrett radii for stable nuclei deduced from muonic atoms. The differences of the model-independent equivalent charge radii $R_{k \alpha}^{\mu}$ between even-even neighboring isotopes (that is, nuclides with $N$ and $N+2$ ) are plotted at the neutron numbers $N+1$. Each symbol is used several times, but in the vicinity of a certain $N$ it always represents one particular element. The lines are drawn to guide the eye. Radii differences are taken from Table $\mathrm{V}$. 

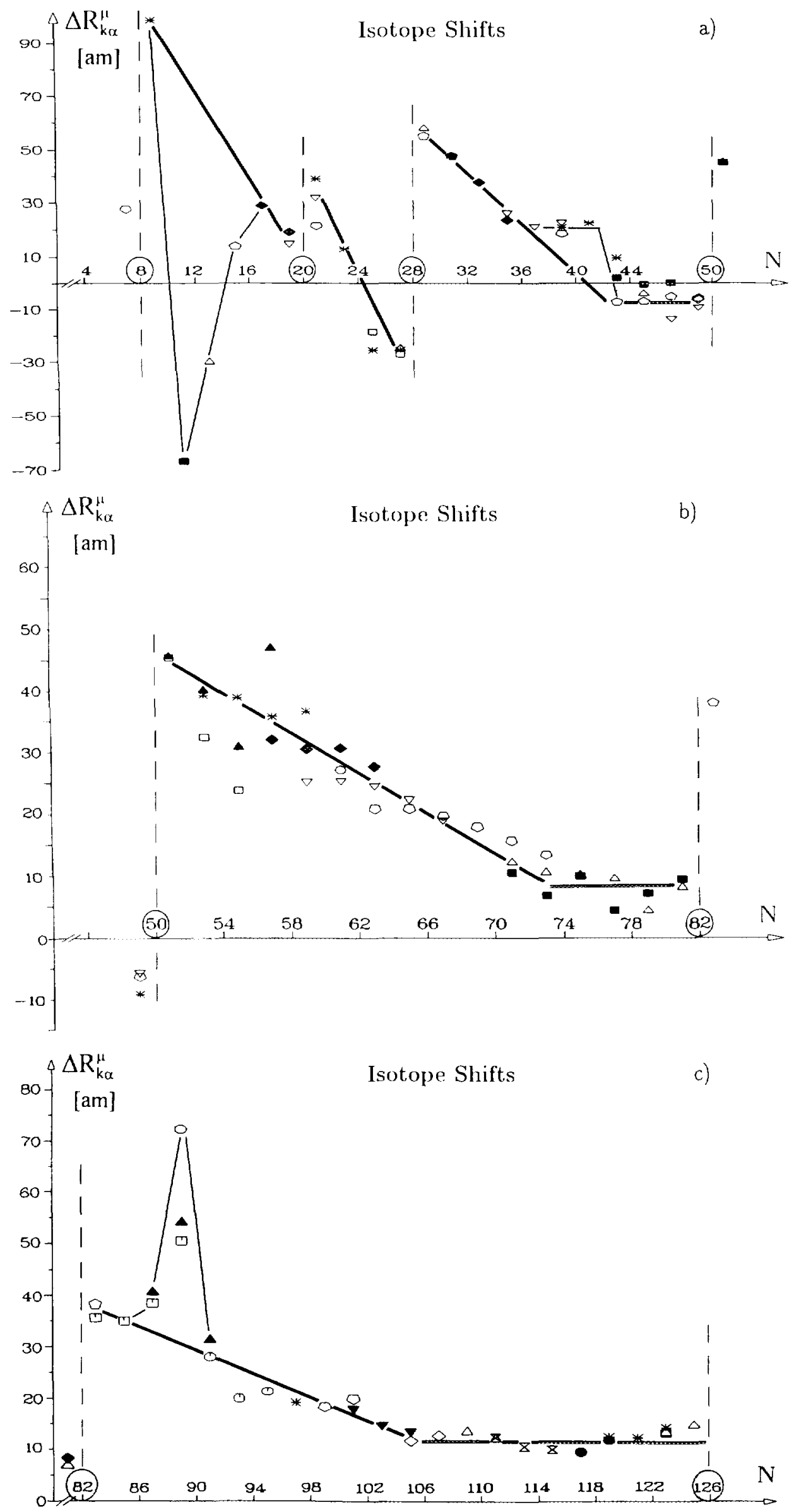

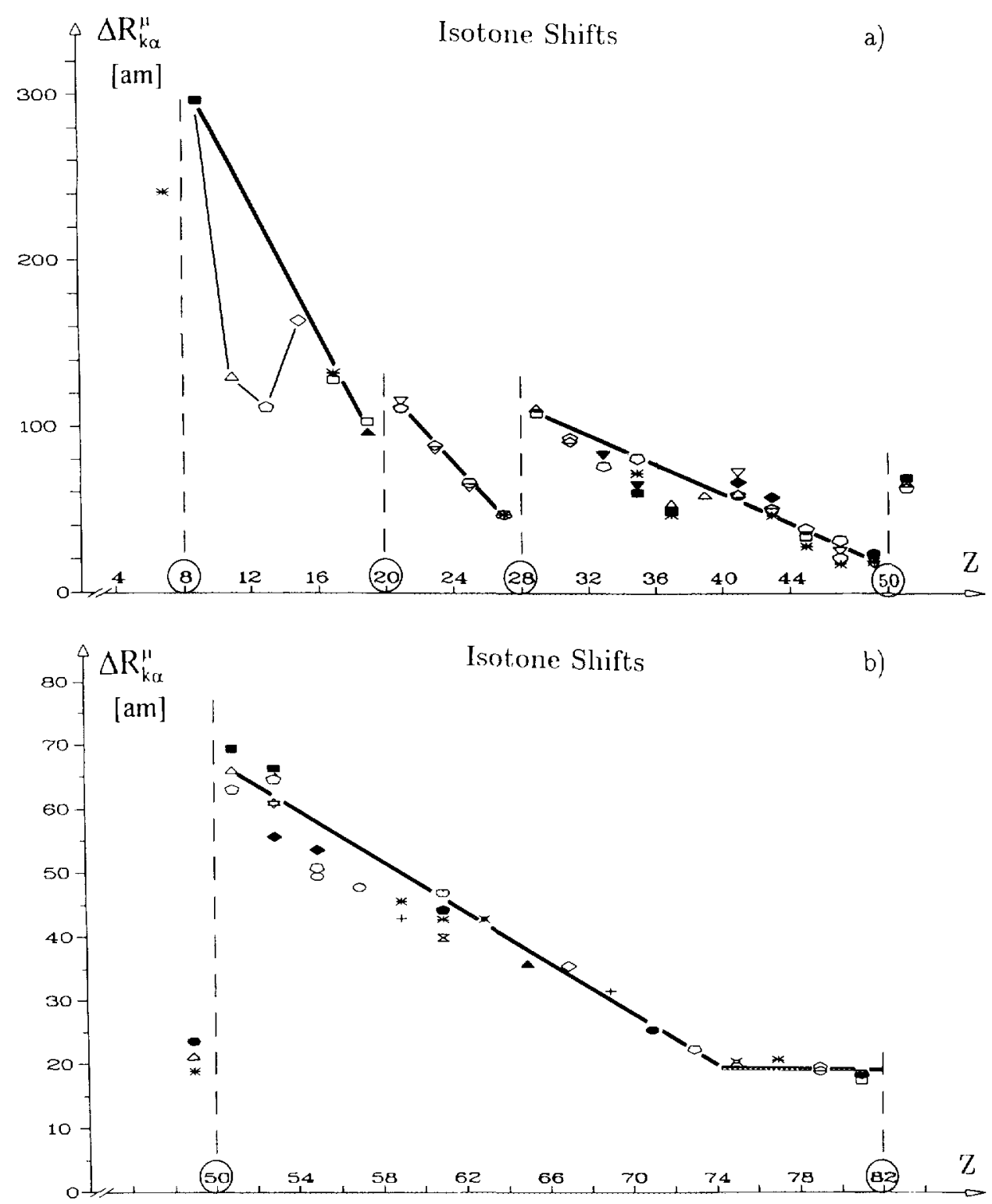

Figure 5. Isotone shifts of Barrett radii deduced from muonic atoms for stable nuclei. The differences of the model-independent equivalent charge radii $R_{k a}^{\mu}$ between even-even neighboring isotones (that is, nuclides with $Z$ and $Z+2$ ) are plotted at the proton numbers $Z+1$. Each shift in the vicinity of a certain $Z$ is marked by a different symbol. The lines are drawn to guide the eye. Radii differences are taken from Table VII.

The accuracy of elastic electron scattering experiments is limited for several reasons:

1. The influence of systematic error sources in elastic electron scattering experiments is relatively large compared to the precision of the other methods as discussed in the following chapter. An error of less than $1 \%$ in the overall normalization of the measured cross sections is difficult to obtain experimentally.
2. An additional error is introduced by the restricted $q$ range accessible to the experiment. The resulting "completeness error" can be estimated by an asymptotic expansion of $F(q)$ for high- $q$ values.

3. The charge distributions might be affected by dispersion corrections which take into account virtual excitations of the nucleus during the scattering process. ${ }^{37}$ By investigating a possible energy dependence of the form factor, the dispersion corrections were studied recently by 
Offermann et al. ${ }^{38}$ at NIKHEF and at MIT. They found that at present the corrections cannot be calculated reliably (see also Friar and Rosén ${ }^{37}$ ). In addition, the assumption made for the value of the cutoff radius $R_{\text {cut }}$ also contributes to the error in the charge distribution $\rho_{\mathrm{N}}(r) .^{35}$

Because of these errors, especially the relatively large systematic uncertainties, the typical accuracy of an rms radius determined by an elastic electron scattering experiment is limited to approximately $0.2 \%$. The electron scattering measurements-as well as the muonic atom investigations-can be applied only for those nuclei for which a sufficient amount of target material is available (at least tens of milligrams). Thus, elastic electron scattering experiments are essentially feasible only for stable isotopes.

On the other hand, electron scattering offers the unique possibility of determining the radial dependence of a charge distribution $\rho_{N}(r)$, whereas all other electromagnetic techniques determine only integral quantities of $\rho_{N}(r)$. Therefore, the charge distribution can be used to connect the different characteristic radial moments determined by different methods. This will be achieved via the ratio of the different radial moments

$$
V_{n}^{\mathrm{e}}=\frac{R_{k \alpha}^{\mathrm{e}}}{\left\langle r^{n}\right\rangle_{\mathrm{e}}^{1 / n}},
$$

which can be calculated approximately one order of magnitude more precisely than the absolute radial moments. As an example, the $V_{n}^{\mathrm{e}}$ factors for the stable lead isotopes have been recently calculated by Mazanek, ${ }^{39}$ which results in a relative error for $V_{2}^{\mathrm{e}}$ of $\delta V_{2}^{\mathrm{e}} / V_{2}^{\mathrm{e}}=1 \times 10^{-4}$. This makes it possible to convert $R_{k \alpha}^{\mu}\left(\delta R_{k \alpha}^{\mu} / R_{k \alpha}^{\mu}=2 \times 10^{-4}\right)$ to the rms radius with almost no loss of accuracy. Thus, elastic electron scattering experiments provide a valuable tool to correlate the precise information obtained by muonic, optical, and $K \mathrm{x}$-ray isotope shift measurements (see Section 6).

\section{Combined Analysis}

The aim of the common analysis of the four methods described above is the determination of the rms radius $\left\langle r^{2}\right\rangle^{1 / 2}$ and the optical factors $F_{i}$ and $M_{i}$. This is possible in a model-independent way as will be shown below. The four methods are sensitive to different radial moments: optical (abbreviated o) and $K \mathrm{x}$-ray $(K-\mathrm{x})$ isotope shifts to $\lambda^{A A^{\prime}}$ (Eq. (2.7)), transitions in muonic atoms $(\mu)$ to the Barrett radius $R_{k \alpha}^{\mu}$ (Eq. (4.3)), and elastic electron scattering (e) to the charge distribution $\rho_{\mathrm{N}}(r)$.

It has been demonstrated in Section 4 that the most precise radius--the Barrett radius $R_{k \alpha}^{\mu}-$ can be deduced from muonic atoms. The Barrett radius can be related to the different radial moments $\left\langle r^{n}\right\rangle^{1 / n}$, with $n=2,4,6$, via the ratio $V_{n}^{\mathrm{e}}=R_{k \alpha}^{\mathrm{e}} /\left\langle r^{n}\right\rangle_{\mathrm{e}}^{1 / n}$ calculated from elastic electron scattering data. The integrand $\rho_{\mathrm{N}}(r) r^{k+2} e^{-\alpha r}$ for the Barrett radius and the integrands $\rho_{\mathrm{N}}(r) r^{n+2}$ for the different radial moments are shown for calcium in Fig. 6. The integrand with $n=2$ and the integrand for the Barrett moment show a very similar radial dependence. Therefore it is not astonishing that the values for the Barrett equivalent radius $R_{k \alpha}$ and the rms equivalent radius $(5 / 3)^{1 / 2}\left\langle r^{2}\right\rangle^{1 / 2}$ are nearly the same. While for lighter nuclei, where $k \approx 2$ and $\alpha \approx 0$, such a behavior is expected, even for lead the deviation for the different radii is only $1 \%$ (see Fig. 7). Specifically, for ${ }^{208} \mathrm{~Pb}, \quad(5 / 3)^{1 / 2}\left\langle r^{2}\right\rangle_{\text {model }}^{1 / 2}$ $=7.106$ and $R_{k \alpha}^{\mu}=7.031$. Evidently most of the systematic errors of an elastic electron scattering experiment will cancel in the ratios $V_{n}^{\mathrm{e}}$. As a consequence, the accuracy of the $\left\langle r^{n}\right\rangle_{\mu \mathrm{e}}^{1 / n}=R_{k \alpha}^{\mu} / V_{n}^{e}$ values is much better than the precision of the corresponding radial moments deduced from elastic electron scattering data alone. The relative accuracy of both $R_{k \alpha}^{\mu}$ and $V_{2}^{e}$ is ${ }^{26}$ about $10^{-4}$. Thus, combining the muonic $R_{k a}^{\mu}$ value with the $V_{2}^{\text {e }}$ ratio yields an rms radius with an error less than twice the error of the Barrett moment. For a detailed error discussion see Refs. 29 and 40.

Once the radial moments of the stable isotopes are determined by such a combined analysis of $\mu$ and $\mathrm{e}$ data, the calculation of the parameters $\lambda_{\mu \mathrm{e}}^{A A^{\prime}}=\delta\left\langle r^{2}\right\rangle^{A A^{\prime}}$ $+\left(C_{2} / C_{1}\right) \delta\left\langle r^{4}\right\rangle^{A A^{\prime}}+\left(C_{3} / C_{1}\right) \delta\left\langle r^{6}\right\rangle^{A A^{\prime}}+\cdots$ for these nuclei is straightforward. The resulting $\lambda_{\mu \mathrm{e}}^{A A^{\prime}}$ values can be compared with the $\lambda_{K-\mathrm{x}}^{A A^{\prime}}$ values derived from $K \mathrm{x}$-ray experiments. The impressive consistency of the two meth-

I(r) Calcium

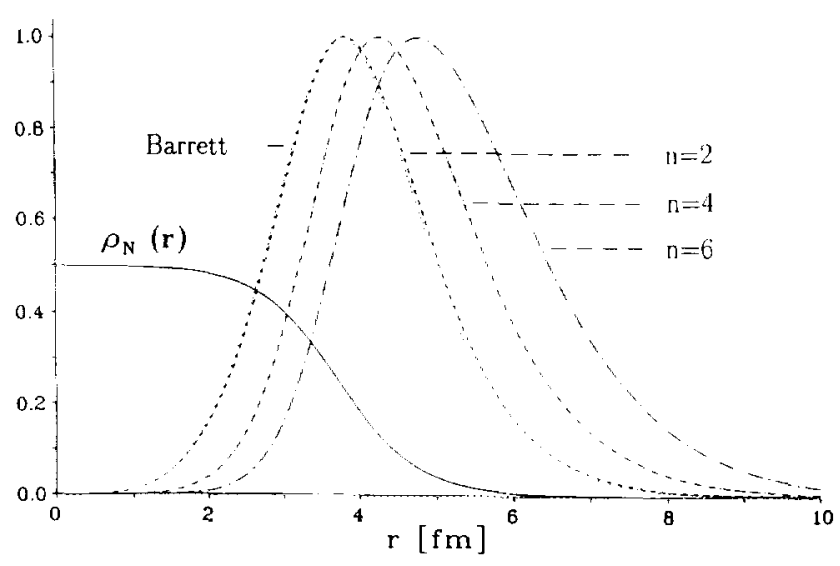

Figure 6. Integrands of the different radial moments $\rho_{\mathrm{N}}(r) r^{n+2}$ and $\rho_{\mathrm{N}}(r) r^{k+2} e^{-a r}$ for calcium. The integrands are normalized to one at the maximum. For comparison the shape of the nuclear charge distribution (solid line) is also shown. 
I(r)

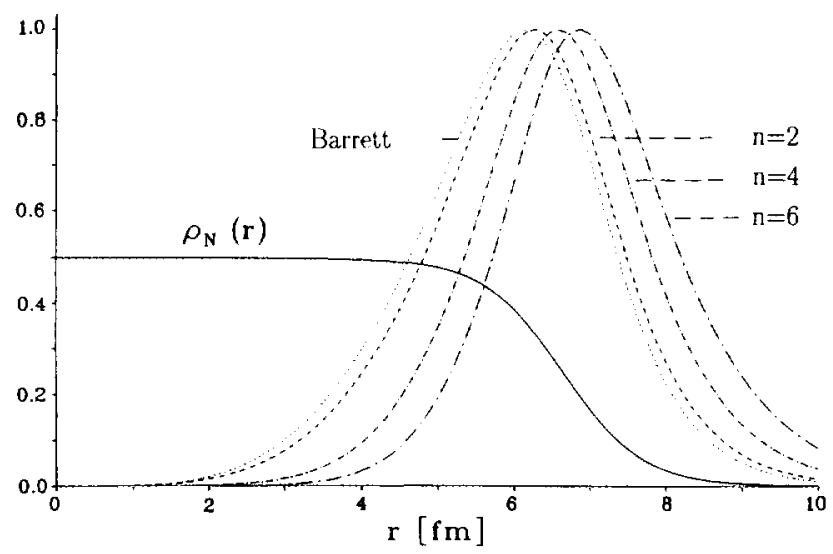

Figure 7. Same as Fig. 6, but for lead.

ods, i.e., $K$-x and $\mu$ plus e, is shown for the lead isotopes in Fig. 1. This supports the concept of calculating $\lambda_{\mu \mathrm{e}}$ from measured data only without any model assumptions. If $\lambda^{A A^{\prime}}$ parameters for at least two isotope pairs are available, either from $\mu$ plus e or from $K$-x experiments, the optical factors $F_{i}$ and $M_{i}$ can be determined by a King plot, using the experimental optical frequency shifts $\delta \nu_{i}^{A A^{\prime}}$ and applying the equation (see Eqs. $(2.1-2.7)$ )

$$
\delta \nu_{i}^{A A^{\prime}}=F_{i} \lambda^{A A^{\prime}}+M_{i} \frac{A-A^{\prime}}{A A^{\prime}} .
$$

In the King plot diagrams in Table $\mathbf{X}$ the reduced IS (see Eq. (7.3)) is plotted on the $y$ axis versus the reduced $\lambda_{\mu \mathrm{e}}^{A A^{\prime}}$ values (see Eq. (7.4)) on the $x$ axis. The reduced IS follows from multiplying Eq. (6.1) by two mass-dependent factors and is given by

$$
\begin{array}{r}
\frac{A_{\text {std }}-A_{\text {std }}^{\prime}}{A_{\text {std }} A_{\text {std }}^{\prime}} \frac{A A^{\prime}}{A-A^{\prime}} \delta \nu_{\text {exp }}^{A A^{\prime}}=\frac{A_{\text {std }}-A_{\text {std }}^{\prime}}{A_{\text {std }} A_{\text {std }}^{\prime}} \frac{A A^{\prime}}{A-A^{\prime}} F_{i} \lambda^{A A^{\prime}} \\
+\frac{A_{\text {std }}-A_{\text {std }}^{\prime}}{A_{\text {std }} A_{\text {std }}^{\prime}} M_{i} .
\end{array}
$$

The multiplication by the factor containing $A_{\text {std }}$ facilitates the reading of the King plot: the intersection of the regression line with the $y$ axis gives the mass shift of the standard pair in the line $i$.

After the factors $F_{i}$ and $M_{i}$ have been determined, the optical experimental frequency shifts of other stable and unstable isotopes can be converted to precise $\lambda^{A A^{\prime}}$ values for the whole isotopic chain. The errors of $F_{i}$ and $M_{i}$ are correlated, as Eq. (6.1) shows. Therefore the $\lambda^{A A^{\prime}}$ values are determined more precisely if the correlation is large. This is the case in the examples given. In order to obtain $\delta\left\langle r^{2}\right\rangle^{A A^{\prime}}$, the $\lambda^{A A^{\prime}}$ parameters must be corrected for the contribution of the differences in the higher radial moments $\left(\delta\left\langle r^{4}\right\rangle, \delta\left\langle r^{6}\right\rangle\right)$. For isotopes where no experimental data on $V_{n}$ factors exist, one can either extrapolate the unknown $V_{n}$ factors from the values of the stable isotopes, or one can use theoretical model calculations ${ }^{41-43}$ or a parametrized charge distribution (Fermi II, Eq. (4.4)). If the $2 p-1 s$ and the $3 d-2 p$ transitions in muonic atoms have been measured, the two parameters of the Fermi II charge distribution, $c$ and $t$, can be determined experimentally (see, for example, Ref. 44). This allows the calculation of $V_{n}^{\mu}$ factors from muonic data alone. The method still uses the Fermi II charge distribution but with both parameters $c$ and $t$ measured. The averaged contribution of the higher radial moments to $\lambda^{A A^{\prime}}$ is given in Fig. 8 (see also Table $X$ ). Finally, with the absolute rms values of the stable isotopes deduced from $\mu$ and e measurements in connection with the $\delta\left\langle r^{2}\right\rangle_{\text {oue }}^{A A^{\prime}}$ values derived from a combined analysis in a King plot, the rms radii of the whole isotope chain can be calculated.

Before giving an example it is useful to recall the conditions for an effective combined analysis:

(i) Precise experimental results for optical isotope shift measurements (with a high percentage of FS) and Barrett radii $R_{k \alpha}^{\mu}$ from muonic atom $2 p-1 s$ transition energies. These data are now available for most isotopes.

(ii) Nuclear charge distributions from elastic electron scattering experiments to calculate the $V_{n}^{\text {e factors, }}$

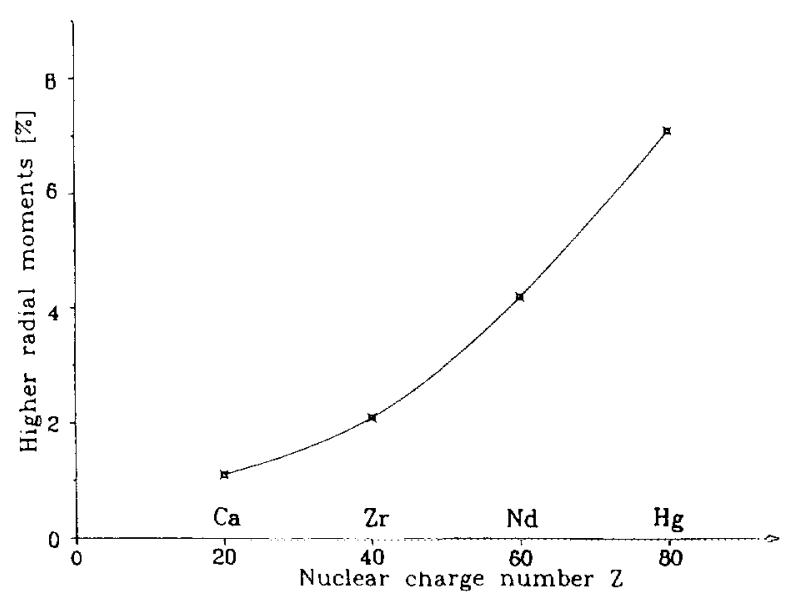

Figure 8. Averaged contribution (in percent) of higher radial moments $\delta\left\langle r^{4}\right\rangle$ and $\delta\left\langle r^{6}\right\rangle$ to the $\lambda$ factor (see Eq. (2.7)). The contribution is always negative, that is, $\lambda<\delta\left\langle r^{2}\right\rangle$. 
which correlate the different radial moments. About half of the stable even isotopes have been measured by the elastic electron scattering method. For odd isotopes, however, only a few data are available because of difficulties caused by contributions from magnetic and/or electric moments and the experimental difficulties of resolving the elastically scattered from the inelastically scattered electrons. This makes it necessary to interpolate the unknown $V_{n}^{\mathrm{e}}$ from values in neighboring isotopes and sometimes between neighboring elements. ${ }^{29} \mathrm{~A}$ justification for this procedure is given below. If the $V_{n}^{e}$ factor has been measured for one isotope only, that same factor may be used for all isotopes. In the cases of the even molybdenum and zirconium isotopes, electron scattering results have shown that the variation of the $V_{2}^{e}$ factors within a chain of isotopes is less than their limits of error (about $10^{-4}$ ). ${ }^{26}$

The input values for $\delta v_{\text {proj }}$ and the $R_{k \alpha}^{\mu}$, the $\Delta R_{k \alpha}^{\mu}$, and the $V_{n}$ values are listed in Tables I, III, V, and X, respectively ( $\delta \nu_{\text {proj }}$ is introduced in Section 2$)$. The procedure and additional assumptions (for $V_{n}$ factors, for example) used for the King plots and the results (Table $\mathrm{X}$ ) are demonstrated below for samarium.

In samarium, elastic electron scattering data are available only for the isotopes $A=148,152$, and 154 (Refs. 45, 46). For these isotopes, the $V_{n}^{\mathrm{e}}$ factors and their errors were calculated by Mazanek, ${ }^{39}$ using the modelindependent Fourier-Bessel analysis. For the other stable isotopes, 144, 147, 149, and 150 , where no electron scattering data exist, the $V_{n}^{\mathrm{e}}$ values have been interpolated. This seems to be adequate, because the $V_{n}^{e}$ factors show only a small variation within the isotopes of one element ${ }^{26}$ and because the consistency of the data is excellent (see the samarium King plot in Fig. 9). All points lie almost perfectly on a straight line, resulting in a $\chi^{2}$ per degree of freedom of 0.08 . This small value indicates that the reported error for the combined $\mu$ plus e data is too large. The error of the $V_{n}^{e}$ factors depends mostly on systematic uncertainties in the elastic scattering data, like normalization, scattering energies, or angles, which are not easy to determine. Also, no correlation between the different isotopes has been taken into account when determining the error of the $V_{n}^{e}$ factors. On the other hand, there is no error matrix available for most of the reported $a_{\nu}$ coefficients. The $V_{n}^{\mathrm{e}}$ can be calculated from the Fourier-Bessel coefficients $a_{\nu}$ (see Table IX) but without the proper error. Therefore it is customary and reasonable to use the King plot parameters $\lambda_{\mu \mathrm{e}}$ deduced assuming no error for the $V_{n}^{e}$ factors. The result for samarium is given in Fig. 10, which still demonstrates an excellent agreement between the $o$ and $\mu$ plus e data, with a $\chi^{2}$ value per degree of freedom close to one. This result also indicates that the error assigned to the $\Delta R_{k \alpha}^{\mu}$ differences due to uncertainties in the nuclear polarization corrections (nuclear polarization; see Section 4) might be regarded as an upper limit

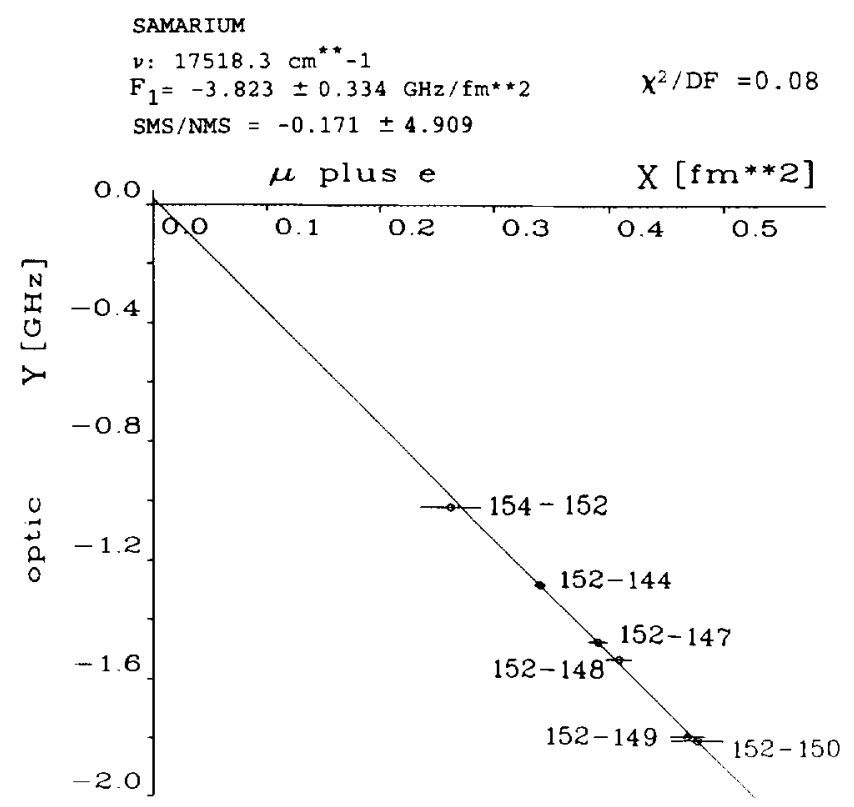

Figure 9. King plot of optical isotope shift measurements against muonic $\mathrm{x}$-ray and elastic electron scattering data for $\mathrm{Sm}$. The error of the $V_{n}^{\mathrm{e}}$ factors (Eq. (5.6)) is included. This results in a too small $\chi^{2}$ of 0.08 per degree of freedom. For definitions of $X$ and $Y$, see Explanation of Table $X$.

and that, as mentioned, the interpolation for the unknown $V_{n}^{\mathrm{e}}$ values seems to be justified. The results of this combined analysis are the optical factors $F_{i}$ and $M_{i}$, which can be compared to values from theoretical calculations (Table XI), and the rms radii (Table XII).

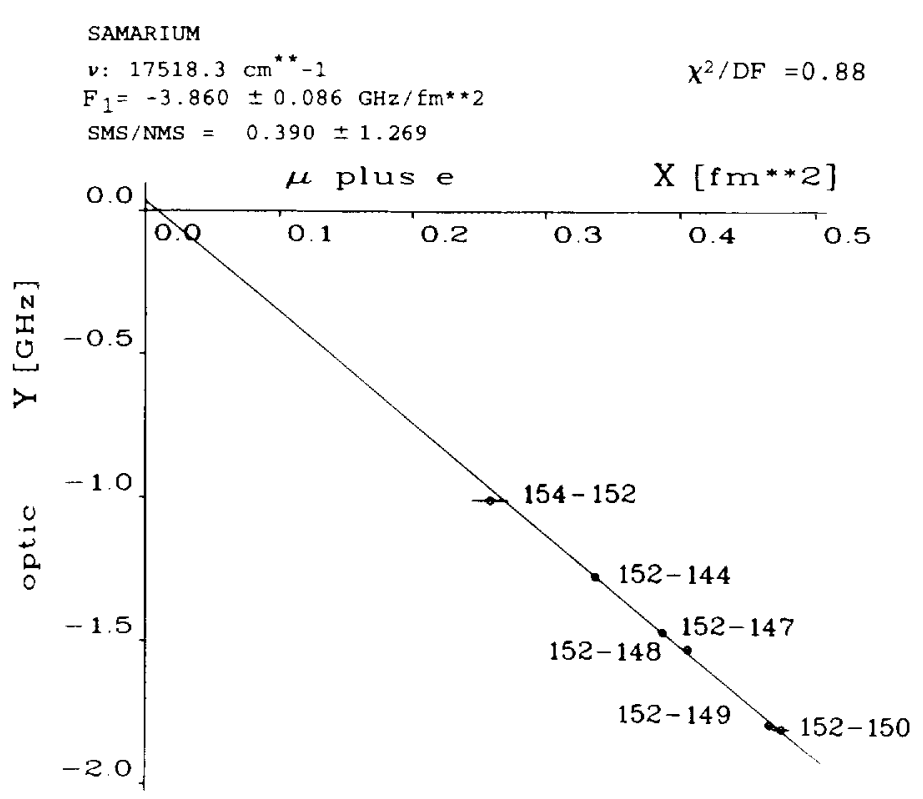

Figure 10. Same as Fig. 9, but without including any error for the $V_{n}^{e}$ factors. The result is a more reasonable $\chi^{2}$ of 0.88 per degree of freedom. 
The final results of the combined analysis are shown in King plots (Table $\mathrm{X}$ ) which illustrate the consistency between the input data from the different experimental methods. Supplementary remarks are given for each element-for example, on the origin of the $V_{n}$ 's-in order to reveal the method of our calculations and to facilitate checks. Many radioactive isotopes have been investigated by optical spectroscopy. As an example, we give in Table $\mathrm{X}$ mean-square radii differences of the unstable lead isotopes as deduced from the regression line established by the stable isotopes. The corresponding mean-square radii are listed in Table XII in order to give an impression for the errors of the radii of the isotopes far from stability. The examples given in this paper should stimulate further experimental and theoretical investigations on the subject. Work on a combined analysis of other elements than those given in Table $\mathrm{X}$ is in progress.

\section{Appendix A: King Plot}

Terminology used in this paper is summarized as follows:

\begin{tabular}{|c|c|}
\hline observed IS: & $\delta \nu_{i}^{A A^{\prime}}=\nu_{i}^{A}-\nu_{i}^{A^{\prime}}$ \\
\hline projected IS: & $\begin{array}{l}\text { the IS in a selected line, } \\
\text { evaluated from the IS } \\
\text { shifts in all relevant lines } \\
\text { by means of a variance- } \\
\text { covariance matrix }\end{array}$ \\
\hline
\end{tabular}

$$
\begin{array}{ll}
\text { scaled ( } \equiv \text { modified) IS: } & \delta \nu_{i}^{A A^{\prime}} \frac{A A^{\prime}}{A-A^{\prime}}=\delta \nu_{i}^{A A^{\prime}} S \\
\text { reduced IS: } & \delta \nu^{A A^{\prime}} S \frac{A_{\text {std }}-A_{\text {std }}^{\prime}}{A_{\text {std }} A_{\text {std }}^{\prime}} \\
\text { reduced } \lambda: & \lambda^{A A^{\prime}} S \frac{A_{\text {std }}-A_{\text {std }}^{\prime}}{A_{\text {std }} A_{\text {std }}^{\prime}},
\end{array}
$$

where $A_{\text {std }}, A_{\text {std }}^{\prime}$ is an arbitrarily chosen standard isotope pair. Projected IS is IS in a selected line, evaluated from IS shifts in all relevant lines by means of a variancecovariance matrix. For sign conventions, see also Appen$\operatorname{dix} B$.

A (two-dimensional) King plot is a diagram where the scaled IS of a line $i$ is plotted on the $y$ axis versus the scaled IS of another line $j$ on the $x$ axis. From Eqs. (2.1), (2.3), and (2.4) follows Eq. (6.1):

$$
\delta \nu_{i}^{A A^{\prime}}=F_{i} \lambda^{A A^{\prime}}+M_{i} \frac{A-A^{\prime}}{A A^{\prime}} .
$$

As a consequence,

(i) the plotted points (= scaled $I^{A A^{\prime}}$ ) must lie on a straight line,

(ii) the slope of the regression line is

$$
F_{i} / F_{j},
$$

(iii) the intersection of the regression line with the $y$ axis is

$$
y=M_{i}-M_{j} F_{i} / F_{j} .
$$

These equations-and consequently the following discussion-imply that the IS is caused by FS and MS only and that any additional isotope-dependent influence on the measured IS has been accounted for. For example, care has to be taken to include second-order hyperfine perturbations.$^{47}$ Another effect is a possible nuclear polarization by the electron. In samarium, the following changes for $\lambda^{A A^{\prime}}$ have been calculated: ${ }^{21}{ }^{150} \mathrm{Sm}-{ }^{148} \mathrm{Sm}, 2 \%$; ${ }^{152} \mathrm{Sm}-{ }^{150} \mathrm{Sm}, 6 \% ;{ }^{154} \mathrm{Sm}-{ }^{152} \mathrm{Sm}, 6 \%$. The excellent agreement between the optical IS measurements (not corrected for nuclear polarization) versus $\mu$ plus e data in the samarium King plot (see Fig. 10) indicates, however, that the nuclear polarization correction for optical transitions may not be in accordance with the calculated values. For other nuclides that are not so heavily deformed as those in the Sm region, a much smaller influence on the IS is expected from theoretical considerations. ${ }^{21}$

Which reference isotope should be used for the plot? As an example, we discuss strontium, an element where MS and FS roughly have about the same size (the nuclear radius decreases with increasing mass number for the stable isotopes). Strontium has four stable isotopes (with $A=84,86,87$, and 88 ) and therefore three independent isotope pairs; the reduced IS of line $\lambda=689.3 \mathrm{~nm}$ is plotted ( $x$ axis) versus the reduced IS of line $\lambda=716.7$ $\mathrm{nm}$ ( $y$ axis) in Fig. 11. The observed shifts in $\mathrm{MHz}$ relative to $A=88$ are, for the $5 s^{2}{ }^{1} S_{0} \rightarrow 5 s 5 p^{3} P_{1} 689.3-\mathrm{nm}$ line, $351.2 \pm 1.8\left(A^{\prime}=84\right), 163.7 \pm 1.0\left(A^{\prime}=86\right), 62.3 \pm 1.4$ $\left(A^{\prime}=87\right)^{48}$ and, for the $5 s 4 d^{1} D_{2} \rightarrow 5 s 6 p^{1} P_{1} 716.7-\mathrm{nm}$ line, $-463.2 \pm 1.0\left(A^{\prime}=84\right),-220.6 \pm 1.0\left(A^{\prime}=86\right)$, $-99.5 \pm 1.0\left(A^{\gamma}=87\right){ }^{49}$

The standard pair, introduced to facilitate the reading of the King-plot diagrams, is arbitrarily chosen to be 88-86, giving a constant factor of $\left(A_{\text {std }}-A_{\text {std }}^{\prime}\right) /$ $\left(A_{\text {std }} A_{\text {std }}^{\prime}\right)=0.000264$ for all plotted strontium values. Therefore the choice of the standard pair is not relevant to the subsequent discussion.

Figure 11 shows five King plots for strontium, where the reference isotope only has been changed from plot to plot. The numerical values of the slopes and $y$ intercepts of the five regression lines are given in Table A.

At first glance it seems surprising that different reference isotopes should yield somewhat different slopes and intersections. The main reason is that the measured values are multiplied by mass-number-dependent factors (see Eq. (7.2)). Therefore different reference isotopes give quite different ranges in the plot (see Fig. 11). The range is introduced as (largest plotted value minus smallest value)/(largest value). In most cases, a large range gives results with the smallest errors. A detailed discussion will be given in a forthcoming paper. ${ }^{50}$ 


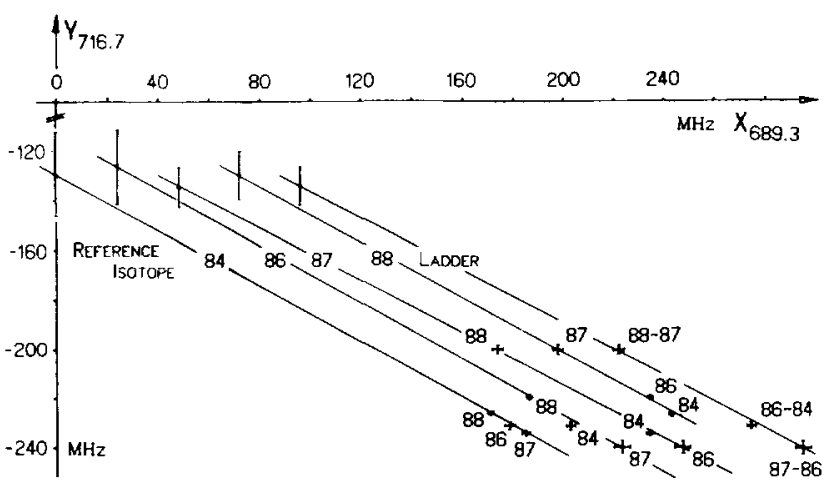

Figure 11. Two-dimensional King plots for two optical strontium lines for five different choices of the reference isotope. The $\mathrm{x}$ axis has been shifted from plot to plot for convenience; the respective $y$ intercepts are indicated by the series of vertical error bars. The corresponding data are given in the text and in Table A. Plotted are the reduced IS in $\mathrm{MHz}$, namely

$$
\begin{aligned}
& X^{A A^{\prime}}=\delta \nu_{889.3 \mathrm{~nm}}^{A^{\prime}} \frac{A A^{\prime}}{A-A^{\prime}} \frac{88-86}{88 \times 86}, \\
& Y^{A A^{\prime}}=\delta \nu_{16.7}^{A A^{\prime}} \mathrm{nm} \frac{A A^{\prime}}{A-A^{\prime}} \frac{88-86}{88 \times 86} .
\end{aligned}
$$

$A, A^{\prime}, 88$, or 86 stands for the more accurate nuclear mass numbers deduced from Ref. 51 .

Nevertheless, an improvement can be achieved, if for each line $i$ the ISs of all possible isotope pairs have been measured (i.e., in strontium, six permutations) and the values for each line are equalized by a matrix. Unfortunately this thorough procedure-often executed in the prelaser days-no longer is en vogue, so most elements had to be treated individually. In this paper a line with a slope (and a corresponding intersection; Eq. (7.6)) with rather small error bars was chosen to serve as a projection line by careful inspection of King plots of all relevant optical lines. When calculating $F_{i}$ and $M_{i}$ factors for other optical lines by means of two-dimensional King plots (see Eqs. (7.5) and (7.6)), at least the same reference isotope (or the ladder) should be used for all lines.

It is not possible to extract $M_{i}$ and $F_{i}$ solely from optical measurements, no matter how many lines and how many isotope pairs have been investigated. However, the $F_{i}$ and $M_{i}$ factors can be found by a special King plot, in which the $\lambda_{\mu \mathrm{e}}^{A A^{\prime}}$ values deduced from muonic and electron scattering data are plotted versus the experimental $\delta \nu_{\text {proj }}^{A A^{\prime}}$ values for one line. If one uses the reduced is (see Eqs. (6.2) and (7.3)) and the reduced $\lambda_{\mu \mathrm{e}}^{A A^{\prime}}$ (see Eq. (7.4)) King plot, the slope gives $F_{i}$ in $\mathrm{GHz} / \mathrm{fm}^{2}$ and the intersection with the $y$ axis (where the optical values are plotted) determines the mass shift for the standard pair in $\mathrm{GHz}$. That procedure is used in this paper.

\section{Appendix B: Sign Convention}

We define $\delta \nu^{A A^{\prime}}$ and $\lambda^{A A^{\prime}}$ in a straightforward way which is in agreement with Otten: ${ }^{5}$

$$
\begin{gathered}
\delta \nu^{A A^{\prime}}=\nu^{A}-\nu^{A^{\prime}} \\
\delta E_{\text {coul }}^{A A^{\prime}}=E_{\text {Coul }}^{A}-E_{\text {Coul }}^{A^{\prime}} \\
\lambda^{A A^{\prime}} \approx \delta\left\langle r^{2}\right\rangle^{A A^{\prime}}=\left\langle r^{2}\right\rangle^{A}-\left\langle r^{2}\right\rangle^{A^{\prime}} \\
\delta \nu_{i, \mathrm{NMS}}^{A A^{\prime}}=\frac{\nu_{i}}{1822.9} \frac{A-A^{\prime}}{A A^{\prime}} .
\end{gathered}
$$

The NMS always shifts the line for the heavier isotope toward larger wavenumbers; this is called a positive shift.

The SMS may be positive or negative, depending on the type of transition and the element.

In many cases the rms radius of the isotopes of an element increases with increasing neutron number. Then $\lambda^{A A^{\prime}}$ is positive, if $A>A^{\prime}$, and the FS in an $s \rightarrow p$ transition (with $s$ the lower and $p$ the upper energy level) is negative:

TABLE A

Numerical Values of the Slopes and Intersections (with the $y$ Axis) of the Five King Plots Drawn in Fig. 11

\begin{tabular}{ccccr}
\hline $\begin{array}{c}\text { Reference } \\
\text { isotope }\end{array}$ & Isotope pairs & Slope $=\frac{F_{716.7}}{F_{689.3}}$ & $\begin{array}{c}\text { Intersection }=\mathrm{MS}^{88-86} \\
{[\mathrm{MHz}]}\end{array}$ & $\begin{array}{c}\text { Range } \\
(\%)\end{array}$ \\
\hline $\mathbf{8 4}$ & $86-84,87-84,88-84$ & $-0.562(96)$ & $-130(17)$ & 7.7 \\
86 & $86-84,87-86,88-86$ & $-0.583(87)$ & $-126(15)$ & 18.2 \\
87 & $87-84,87-86,88-87$ & $-0.533(44)$ & $-135(8)$ & 36.9 \\
$\mathbf{8 8}$ & $88-84,88-86,88-87$ & $-0.559(57)$ & $-130(10)$ & 26.4 \\
Ladder & $86-84,87-86,88-87$ & $-0.583(50)$ & $-134(8)$ & 36.9 \\
\hline
\end{tabular}


the line for the heavier isotope is shifted toward smaller wavenumbers. Therefore $F_{i}$ for an $s \rightarrow p$ transition (with $s$ the lower, and $p$ the upper energy level) is negative. For a $p \rightarrow s$ transition, $F_{i}$ is positive.

\section{Acknowledgments}

This work was supported by the Bundesministerium für Forschung und Technologie, Germany, and by the Swiss National Foundation. We thank B. Fricke, Universität Kassel, for MCDF calculations of optical factors. One of us (C.B.) is indebted to the Institut de Physique de l'Université de Fribourg for its friendly hospitality.

\section{References for the Introduction}

1. P. Aufmuth, K. Heilig, and A. Steudel, Atomic DATA AND NUClEAR DATA TABles 37, 455 (1987)

2. F. Boehm and P. L. Lee, ATOMIC Data AND NuClear data TABLES 14, 605 (1974)

3. R. Engfer, H. Schneuwly, J. L. Vuilleumier, H. K. Walter, and A. Zehnder, ATOMIC DATA AND NUCLEAR DATA TABLES 14, 509 (1974)

4. H. de Vries, C. W. de Jager, and C. de Vries, AtomiC Data and Nuclear Data Tables 36, 495 (1987)

5. E. W. Otten, in Treatise on Heavy Ion Physics, edited by D. A. Bromley (Plenum, New York, 1987), Vol. 8 , p. 517

6. K. Heilig, Spectrochim. Acta B 32, 1 (1977); 37, 417 (1982); 42, 1237 (1987); 47, 303 (1991)

7. R. C. Barrett, Phys. Lett. B 33, 388 (1970)

8. K. Heilig and A. Steudel, Atomic Data AND NuClear Data TABLES 14, 613 (1974)

9. W. H. King, Isotope Shifts in Atomic Spectra, Physics of Atoms and Molecules Series, edited by P. G. Burke and H. Kleinpoppen (Plenum, New York/London, 1984)

10. E. Lindroth, A. M. Mårtensson-Pendrill, and S. Salomonson, Phys. Rev. A 31, 58 (1985)

11. E. C. Seltzer, Phys. Rev. 188, 1916 (1969)

12. S. A. Blundell, P. E. G. Baird, C. W. P. Palmer, D. N. Stacey, and G. K. Woodgate, J. Phys. B 20, 3663 (1987)

13. K. Heilig, Hyperfine Interact. 38, 803 (1987)

14. F. A. Babushkin, Sov. Phys. JETP 15, 1113 (1962); JETP 17, 1118 (1963)

15. D. Zimmermann, Z. Phys. A 315, 123 (1984); 321, $23(1985)$
16. Z. Fang, O. Redi, and H. H. Stroke, J. Phys. II France 2, 877 (1992)

17. G. Torbohm, B. Fricke, and A. Rosén, Phys. Rev. A 31, 2038 (1985)

18. B. Fricke, private communication, Universität Kassel, 1993

19. C. W. P. Palmer, P. E. G. Baird, S. A. Blundell, J. R. Brandenberger, C. J. Foot, D. N. Stacey, and G. K. Woodgate, J. Phys. B 17, 2197 (1984)

20. K. Wendt, S. A. Ahmad, F. Buchinger, A. C. Mueller, R. Neugart, and E. W. Otten, Z. Phys. A 318, 125 (1984)

21. B. Hoffmann, G. Baur, and J. Speth, Z. Phys. A 315, 57 (1984)

22. R. B. Chesler and F. Boehm, Phys. Rev. 166, 1202 (1968)

23. G. L. Borchert, O. W. B. Schult, J. Speth, P. G. Hansen, B. Jonson, H. L. Raven, and J. B. McGrory, Nuovo Cimento A 73, 273 (1983)

24. P. L. Lee and F. Boehm, Phys. Rev. 8, 819 (1973)

25. G. Fricke, C. Bernhardt, G. Mallot, Th. Hennemann, J. Herberz, L. A. Schaller, L. Schellenberg, and E. B. Shera, Confit, Institute Report, Institut für Kernphysik, KPH 16/93, Universität Mainz, 1993

26. P. Mazanek, Diplomarbeit, Institut für Kernphysik, KPH 11/89, Universität Mainz, 1989

27. G. A. Rinker, private communication, LANL, Los Alamos, NM, 1976

28. C. S. Wu and L. Wilets, Annu. Rev. Nucl. Sci. 19, 527 (1969)

29. C. Bernhardt, Ph.D. thesis, Institut für Kernphysik, KPH 6/92, Universität Mainz, 1992

30. G. Fricke, J. Herberz, Th. Hennemann, G. Mallot, L. A. Schaller, L. Schellenberg, C. Piller, and R. JacotGuillarmod, Phys. Rev. C 45, 80 (1992)

31. H. J. Emrich, G. Fricke, M. V. Hoehn, K. Kaeser, G. Mallot, H. Miska, B. Robert-Tissot, D. Rychel, L. A. Schaller, L. Schellenberg, H. Schneuwly, E. B. Shera, H. G. Sieberling, R. M. Steffen, H. D. Wohlfahrt, and Y. Yamazaki, Proceedings, 4th International Conference on Nuclei far from Stability, $\mathrm{Hel}$ singor, Denmark, 1981, edited by L. O. Skolen, p. 33

32. L. A. Schaller, Z. Phys. C 56, 48 (1992)

33. K. Merle, Ph.D. thesis, Institut für Kernphysik, KPH 20/76, Universität Mainz, 1976 
34. L. R. B. Elton, Nuclear Sizes (Oxford Univ. Press, London, 1961)

35. B. Dreher, J. Friedrich, K. Merle, H. Rothaas, and G. Lührs, Nucl. Phys. A 235, 219 (1974)

36. I. Sick, Nucl. Phys. A 218, 509 (1974)

37. J. L. Friar and M. Rosén, Ann. Phys. (N.Y.) 87, 289 (1974)

38. E. A. J. M. Offermann, L. S. Cardman, C. W. de Jager, H. Miska, C. de Vries, and H. de Vries, Phys. Rev. C 44, 1096 (1991)

39. P. Mazanek, Ph.D. thesis, Institut für Kernphysik, KPH 5/92, Universität Mainz, 1992

40. J. Herberz, Ph.D. thesis, Institut für Kernphysik, KPH 6/89, Universität Mainz, 1989

41. M. M. Sharma, G. A. Laladisis, and P. Ring, Phys. Lett. B 317, 9 (1993)

42. J. Friedrich and P.-G. Reinhard, Phys. Rev. C 33, 335 (1986)

43. B. Nerlo-Pomorska, K. Pomorski, and B. SkorupskaMach, Nucl. Phys. A 562, 180 (1993)
44. E. B. Shera, H. D. Wohlfahrt, M. V. Hoehn, and Y. Tanaka, Phys. Lett. B 112, 124 (1982)

45. R. Hofmann, Ph.D. thesis, Institut für Kernphysik, KPH 80, Universität Mainz, 1980

46. X. H. Phan, H. G. Andresen, L. S. Cardman, J.-M. Cavedon, J.-C. Clemens, B. Frois, M. Girod, D. Gogny, D. Goutte, B. Grammaticos, R. Hofmann, M. Huet, P. Leconte, S. K. Platchkov, I. Sick, and S. E. Williamson, Phys. Rev. C 38, 1173 (1988)

47. H. Kopfermann, Kernmomente, Akad. Verlagsges, Frankfurt/Main, 1956; Nuclear Moments (Academic Press, New York, 1958)

48. D. Bender, H. Brand, and V. Pfeufer, Z. Phys. A 318, 291 (1984)

49. P. Grundevik, M. Gustavsson, I. Lindgren, G. Olsson, T. Olsson, and A. Rosén, Z. Phys. A 311, 143 (1983)

50. K. Heilig, to be published

51. A. H. Wapstra and G. Audi, Nucl. Phys. A 432, 1 (1985) 


\section{EXPLANATION OF TABLES}

\section{TABLE I. Observed (Projected) Optical Isotope Shifts of Selected Elements}

Only optical isotope shifts for those elements and isotopes for which a combined analysis has been made (see Table $\mathrm{X}$ ) are listed.

$Z$ element

The transition is listed below the atomic number and element designation. Wavelengths given are in air, and wavenumbers in vacuum. The frequency graph

A

$\delta \nu_{\text {proj }}$

references shows isotope shifts relative to the reference isotope.

Atomic weight, shorthand notation; for all calculations the more accurate values for the nuclear masses from Wapstra and Audi ${ }^{51}$ were used.

Observed optical isotope shift (IS) relative to the indicated reference isotope, projected onto one transition per element (see Section 2 and Appendix A). Errors are given in parentheses. When used in the combined analysis, IS is taken as positive when the transition of the heavier isotope is shifted toward higher frequencies (see Appendix B).

Source of experimental data, keyed to the list of References for Tables.

TABLE II. Observed $K \alpha$ X-Ray Isotope Shifts $\delta E$ and Charge Radius Variations $\lambda$

Isotope Pair

$\delta E_{C o u}^{A A^{\prime}}$

$-\lambda^{A A^{\prime}}$

Ref.
Element symbol and isotope pair $A-A^{\prime}, A$ being the lighter isotope.

Observed energy shift of the $2 p_{3 / 2} \rightarrow 1 s_{1 / 2} K \alpha_{1}$ x-ray transition, in meV, corrected for the mass shift (see Refs. 2 and 22). The errors are standard deviations.

$$
\begin{aligned}
\delta E_{\text {Coul }}^{A A^{\prime}} & =E_{\text {Coul }}(A)-E_{\text {Coul }}\left(A^{\prime}\right) \\
-\delta E_{\text {Coul }}^{A A^{\prime}} & =C_{1} \delta\left\langle r^{2}\right\rangle^{A A^{\prime}}+C_{2} \delta\left\langle r^{4}\right\rangle^{A A^{\prime}}+C_{3} \delta\left\langle r^{6}\right\rangle^{A A^{\prime}}+\cdots
\end{aligned}
$$

The $C_{i}$ are Seltzer coefficients (Refs. 2, 11, and 12). $\delta\left\langle r^{2}\right\rangle^{A A^{\prime}}=\left\langle r^{2}\right\rangle^{A}-\left\langle r^{2}\right\rangle^{A^{\prime}}$.

$\lambda^{A A^{\prime}}=-\delta E_{\text {Coul }}^{A A^{\prime}} / C_{1}$ (see Eq. 2.7). In this Table and the equations above, the lighter isotope always appears first. Hence $\lambda$ has an opposite sign from $\delta E$.

Source of experimental data, keyed to the list of References for Tables.

TABLE IIIA. Muonic $2 p \rightarrow 1 s$ Transition Energies and Barrett Radii for $Z<60$ and $Z>77$

$E_{\text {exp. }}$

Experimental energy taken from a comprehensive matrix error analysis procedure [Fr93a], if not stated otherwise. If two energies are listed, the first (second) value given corresponds to the $2 p_{1 / 2} \rightarrow 1 s_{1 / 2}$ $\left(2 p_{3 / 2} \rightarrow 1 s_{1 / 2}\right)$ transition. Otherwise the given value corresponds to the center of gravity of the $2 p \rightarrow 1 s$ transition, or, for some elements, to the $\left(2 p_{3 / 2} \rightarrow\right.$ $1 s_{1 / 2}$ ) transition as indicated. The statistical error in the last quoted digits is listed below each energy. 


\section{EXPLANATION OF TABLES continued}

$E_{\text {theo. }}$

NPol

c

$\left\langle r^{2}\right\rangle_{\text {model }}^{1 / 2}$
$\alpha, \mathrm{k}$
$C_{z}$
$R_{k \alpha}^{\mu}$

Ref.
Energy of the transition calculated with a two-parameter Fermi distribution (Eq. (4.4)),

$$
\rho(r)=\rho_{0}\left(1+\exp \left[\frac{r-c}{a}\right]\right)^{-1},
$$

including QED and nuclear polarization corrections. The skin thickness $t=4 a \ln 3$ is fixed at $2.30 \mathrm{fm}$, whereas the half-density radius $c$ is fitted to reproduce the experimental transition energy.

Calculated nuclear polarization correction ([Ri76], [Ri78]). The nuclear data for these calculations are taken from Nuclear Data Sheets up to 1992.

Half-density parameter $c$ of the two-parameter Fermi distribution. The error in the last digits listed below each value is the fit error only and does not include model-dependent effects.

Rms value of the charge radius (Eq. (1.2)) calculated using the two-parameter Fermi distribution with $t=2.30 \mathrm{fm}$.

Parameters of the Barrett moment fitted to reproduce the differences of the muon potentials in the initial and final state.

Sensitivity factor $C_{z}=d R_{k \alpha}^{\mu} / d E$ in am $/ \mathrm{eV}$ or am $/ \mathrm{keV}$ as indicated. $1 \mathrm{am}=10^{-18} \mathrm{~m}$.

Model-independent Barrett equivalent radius calculated from Eq. (4.3) with the parameters $k, \alpha$ fitted to the corresponding transition energy. The first error corresponds to statistical errors but not to systematical errors. The second error is due to uncertainties in calculating the nuclear polarization correction. This error was conservatively estimated to be $30 \%$ of the total nuclear polarization value ([Ri76], [Ri78]).

Source of experimental data, keyed to the list of References for Tables.

TABLE IIIB. Muonic $2 p \rightarrow 1 s$ Transition Energies and Relative Intensities of Deformed Nuclei with $60 \leqslant Z \leqslant 77$

\section{Transition}

Initial state

Final state

$E_{\text {exp. }}$
The coupled muon/nucleus states which form the major component of the wave functions of the initial and final state involved in each transition. Listed are the muon orbital followed by the spin parity of the nuclear state, $I^{\pi}$, and the hyperfine level.

Experimental energy of the listed hyperfine component. The given error includes statistical errors and the fit error. Since an entire hyperfine structure complex was fitted and not just a single component, every component of such a complex has the same experimental error independent of the intensity of the component. 


\section{EXPLANATION OF TABLES continued}

$E_{\text {theo. }}$

Relative Intensity
Energy of the listed hyperfine component calculated with a deformed Fermi distribution including QED and nuclear polarization corrections. The charge density distribution parameters and the nuclear polarization corrections are listed in Table IIIC.

Intensity relative to the strongest component, which is listed as 1.000 .

This table includes static and dynamic hyperfine components measured in nuclei between ${ }^{150} \mathrm{Nd}$ and ${ }^{193} \mathrm{Ir}$, which in general have not been published before. For nuclei with an even (odd) number of nucleons, the $10(20)$ strongest components are listed. All values in this table are taken from the references quoted in Table IIIC.

TABLE IIIC. Barrett Radii and Related Parameters of Deformed Nuclei with $60 \leqslant Z \leqslant 77$

NPol

$R_{0}, \mathrm{a}, \beta_{2}$

$\left\langle r^{2}\right\rangle_{\text {model }}^{1 / 2}$

$\alpha, \mathbf{k}$

$C_{z}$

$R_{k \boldsymbol{\alpha}}^{\mu}$

Calculated nuclear polarization correction ([Ri76], [Ri78]) for the center of gravity of the $2 p_{1 / 2} \Rightarrow$ $1 s_{1 / 2}$ (upper value) and the $2 p_{3 / 2} \rightarrow 1 s_{1 / 2}$ (lower value) hyperfine components found in Table IIIB and/or in the quoted references.

Parameters of the deformed Fermi distribution,

$$
\rho(r)=\rho_{\text {Norm }}\left(1+\exp \left[\frac{r-R_{0}\left[1+\beta_{2} Y_{20}(\theta, \phi)\right]}{a}\right]\right)^{-1} .
$$

$\rho_{\text {Norm }}$ is determined by normalizing the total charge to $\mathrm{Ze}$.

Rms value of the charge radius calculated from $R_{0}, a$, and $\beta_{2}$.

Same as in Table IIIA.

Same as in Table IIIA.

Model-independent Barrett equivalent radius (Eq. (4.3)). Errors in the last quoted digits given in parentheses below each value are obtained with the help of the sensitivity factors $C_{z}$. The first error listed corresponds to the statistical and fit error of $E_{\text {exp }}$ for each complex. The second error, corresponding to uncertainties in calculating the nuclear polarization corrections, was estimated as follows: A $28 \%$ error was assigned to the calculation of the contribution of the high-lying nuclear states to the total nuclear polarization correction. The error for the calculation of the contribution of the low-lying states was estimated to be $2 \%$, resulting essentially from the errors of the transition probabilities $B(E 2)$ [Ri76].

Ref. Source of tabulated data, keyed to the list of References for Tables. 


\section{EXPLANATION OF TABLES continued}

TABLE IV. Confit Matrices for Muonic $2 p \rightarrow 1 s$ Energy Differences of Isotopes, $31 \leqslant Z \leqslant 58$

In this table the energy differences (in $\mathrm{keV}$ ) of isotopes resulting from a comprehensive matrix error analysis procedure are given [Fr93a]. The upper right half shows the energy difference for the $2 p_{1 / 2} \rightarrow 1 s_{1 / 2}$ transition between the lighter and the heavier nucleus, and in the lower left half is the difference for the $2 p_{3 / 2} \rightarrow 1 s_{1 / 2}$ transition between the heavier and the lighter nucleus. The quoted errors include statistical but no systematic errors.

TABLE V. Differences of Barrett Radii for Isotopes, $6 \leqslant Z \leqslant 82$

$\Delta R_{k \alpha}^{\mu}$

Difference of the model-independent equivalent radius (in am $=10^{-18} \mathrm{~m}$ ) based on the energy differences given in Table IV and the parameters $\alpha$ and $k$ in Table IIIA or Table IIIC.

In this table the value for the heavier minus the lighter nucleus is given. The first error is derived from the error of the experimental energy, whereas the second one results from uncertainties in calculating the nuclear polarization corrections. As an upper limit, this second error was estimated assuming a $10 \%$ error for the larger of the nuclear polarization corrections of the two isotopes (see Table IIIA). For deformed nuclei (see Table IIIC), $\frac{1}{3}$ of the larger error of the two isotopes-as assigned to the absolute values of $R_{k \alpha}^{\mu}$-is given [Ri76].

TABLE VI. Confit Matrices for Muonic $2 p \rightarrow 1 s$ Energy Differences of Isotones, $10 \leqslant N \leqslant 82$

See explanation for Table IV.

TABLE VII. Differences of Barrett Radii for Isotones, $8 \leqslant N \leqslant 126$

Same explanation as for Table $\mathrm{V}$, but based on the energy differences given in Table VI and the parameters $\alpha$ and $k$ in Tables IIIA or IIIC. Shifts between isotones which have not been measured simultaneously are marked by an asterisk. In these cases the given experimental error is just the quadratic sum of the errors assigned to the absolute values of the $R_{k \alpha}^{\mu}$ listed in Table IIIA or Table IIIC. Not all measured isotone shifts have been listed. Those values can be deduced in combination with Table $\mathrm{V}$.

\section{TABLE VIII. Charge Density Distribution Parameters from Elastic Electron Scattering}

Data published subsequent to Ref. 4, from 1987 until 1993, are given in this Table. The normalization of the charge distribution is such that $4 \pi \int \rho(r) r^{2} d r=\mathrm{Ze}$. model

Model-independent analysis by means of a Fourier-Bessel expansion for the charge distribution.

SOG Model-independent analysis by means of an expansion for the charge distribution as a sum of Gaussians. 


\section{EXPLANATION OF TABLES continued}

$2 \mathrm{pF}$

$3 \mathrm{pF}$

$2 \mathrm{pG}$

$3 p G$

$\left\langle r^{2}\right\rangle^{1 / 2}$

$\mathrm{c}, \mathrm{a}, \mathrm{w}$

q-range

Ref.
Two-parameter Fermi model:

$$
\rho(r)=\rho_{0} /(1+\exp ((r-c) / a)) .
$$

Three-parameter Fermi model:

$$
\rho(r)=\rho_{0}\left(1+w r^{2} / c^{2}\right) /(1+\exp ((r-c) / a)) .
$$

Two-parameter Gaussian model:

$$
\rho(r)=\rho_{0} /\left(1+\exp \left(\left(r^{2}-c^{2}\right) / a^{2}\right)\right) .
$$

Three-parameter Gaussian model:

$$
\rho(r)=\rho_{0}\left(1+w r^{2} / c^{2}\right) /\left(1+\exp \left(\left(r^{2}-c^{2}\right) / a^{2}\right)\right) .
$$

Rms radius of the charge distribution, Eq. (1.2).

Parameters of the given charge distribution.

The momentum-transfer range covered by the data used in the analysis.

Source of the tabulated data, keyed to the list of references following the Tables.

\section{TABLE IX. Fourier-Bessel Coefficients from Elastic Electron Scattering}

Data published subsequent to Ref. 4, from 1987 until 1993, are given in this Table.

$\left\langle r^{n}\right\rangle^{1 / n}, n=2,4,6$ Radial moments of the charge distribution, Eq. (1.3).

$R_{k \alpha}^{e}$

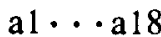

$\boldsymbol{R}_{c u}$

Ref.
Barrett equivalent radius calculated from the given charge distribution with $\alpha$ and $k$ taken from Table IIIA.

List of the Fourier-Bessel coefficients $a_{\nu}$, with $\nu=1$ up to 18 . The coefficients are defined by

$$
\begin{array}{ll}
\rho(r)=\sum_{\nu} a_{\nu} j_{0}\left(\nu \pi r / R_{\text {cut }}\right) & \text { for } r \leqslant R_{\text {cut }} \\
\rho(r)=0 & \text { for } r>R_{\text {cut }}
\end{array}
$$

The normalization is chosen such that

$$
4 \pi \int \rho(r) r^{2} d r=\mathrm{Ze}
$$

The momentum-transfer range ( $q$ range) covered by the data used in the analysis is given in Table VIII. Value of the cutoff radius, beyond which the charge density is assumed to be zero.

Source of the tabulated data, keyed to the list of references following the Tables.

TABLE X. King Plots: Optical versus Combined Muonic and Elastic Electron Scattering Data

The plots in this Table refer to the same element and transition combinations as in Table I.

$\mathrm{R}_{\mathrm{k} \alpha}^{\mu}\left(A_{\mathrm{ref}}\right)$ $\mathrm{C}_{\mathrm{i}} / \mathrm{C}_{1}$
Barrett radius of the reference isotope.

Ratios of Seltzer coefficients (see Eq. (2.7)). 


\section{EXPLANATION OF TABLES continued}

$\lambda_{\text {proj }}$

$\mathrm{F}_{\boldsymbol{i}}$

$\mathrm{FS}^{A-A^{\prime}}$

$\mathrm{MS}^{A-A^{\prime}}$

$\mathrm{NMS}^{A-A^{\prime}}$

$\mathrm{SMS}^{A-A^{\prime}}$

$\chi^{2} /$ D.F.

$\mathrm{V}_{n}, n=2,4,6$

$\delta\left\langle\mathbf{r}^{2}\right\rangle_{\mathrm{o} \mu \mathrm{e}}$

HM

$x$ axis: $X_{\mu \mathrm{e}}$

$y$ axis: $Y_{\text {optic }}$
Wavelength of the selected optical line against which all other optical lines have been projected (see Section 2 and Table I).

Electronic factor for the transition $i$ (see Eqs. (2.6) and (6.1)).

Field shift for the standard isotope pair, that is, between isotopes $A=A_{\text {std }}$ and $A^{\prime}=A_{\text {std. }}^{\prime}$.

Total mass shift for the standard isotope pair.

Normal mass shift for the standard isotope pair.

Specific mass shift for the standard isotope pair.

Chi squared per degree of freedom for the combined analysis.

$V_{n}$ values (see Eq. (5.6) for definition and see Note at the end of each plot). The $V_{n}$ factors are used without error (see Section 6). Superscript e on the mass number of the isotope indicates $V_{n}$ values derived from experiment.

Differences of the mean-square radii $\left(=\left\langle r^{2}\right\rangle^{A}-\left\langle r^{2}\right\rangle^{A^{\prime}}\right)$ resulting from the combined analysis for the isotope pair $A-A^{\prime}$.

Contribution of the higher radial moments $\left\langle r^{4}\right\rangle$ and $\left\langle r^{6}\right\rangle$ to the nuclear $\lambda$ parameter (see Eq. (2.7)). HM is always negative; therefore $\lambda\left\langle\delta\left\langle r^{2}\right\rangle\right.$.

Plotted is the reduced $\lambda$ (Eq. (7.4))

$$
\lambda_{\mu \mathrm{e}}^{A A^{\prime}} \frac{A_{\text {std }}-A_{\text {std }}^{\prime}}{A_{\text {std }} A_{\text {std }}^{\prime}} \frac{A A^{\prime}}{A-A^{\prime}},
$$

with $\lambda_{\mu \mathrm{e}}^{A A^{\prime}}$ taken from combined muonic and electron scattering data.

Plotted is the reduced IS (Eq. (7.3))

$$
\delta \nu_{i}^{A A^{\prime}} \frac{A_{\mathrm{std}}-A_{\mathrm{std}}^{\prime}}{A_{\mathrm{std}} A_{\mathrm{std}}^{\prime}} \frac{A A^{\prime}}{A-A^{\prime}},
$$

with $\delta \nu_{i}^{A A^{\prime}}$ the measured, projected optical isotope shift between isotopes $A$ and $A^{\prime}$. The slope of the regression line gives the electronic factor $F_{i}$. The intersection of the line with the $y$ axis gives the MS for the standard pair: $\delta \nu_{i, \mathrm{MS}}^{A_{\text {sdd }} A^{\prime} \text { 'std }}$ (see Eq. (2.3)). Subtracting the calculated NMS from the MS determines the SMS.

TABLE XI. Electronic Factor $F$ and Specific to Normal Mass Shift Ratio SMS/NMS for Projected Optical Lines

$\lambda$

type of transition

SMS/NMS
Wavelength of the optical reference line (see Tables I and $\mathrm{X})$ for the indicated element.

Orbital designations for lower $\rightarrow$ upper state.

Ratio of specific to normal mass shift for the optical reference line. 


\section{EXPLANATION OF TABLES continued}

$F_{i}$

calc. (MCDF)

Electronic factor $F_{i}$ (see Eqs. (2.6) and (6.1)) for the given optical line.

Electronic factor based on multiconfiguration Dirac-Fock calculations. The errors, when quoted, result from an optical King plot (see Appendix) where the line used in the combined analysis is projected on the line for which MCDF calculations are made. For the above three sets of data, errors in the last quoted digits are given in parentheses following the respective values.

Ref. Source of MCDF calculation, keyed to the list of References for Tables.

Semiempirical values for the electronic factor can be found in [Au87] and references therein.

TABLE XII. Root-Mean-Square Charge Radii from the Combined Analysis of Optical, Muonic, and Elastic Electron Scattering Data

$\left\langle r^{2}\right\rangle_{\text {oue }}^{1 / 2}$

$\Delta\left\langle r^{2}\right\rangle^{1 / 2}$
$\mathrm{Rms}$ radius from a combined analysis of optical (o), muonic $(\mu)$, and electron (e) scattering data. The error for the last digits, given in parentheses, does not include uncertainties from the $V_{n}$ factors.

Difference between the rms radius resulting from electron scattering and muonic data and the rms radius from the combined analysis:

$$
\Delta\left\langle r^{2}\right\rangle^{1 / 2}=\left\langle r^{2}\right\rangle_{\mu \mathrm{e}}^{1 / 2}-\left\langle r^{2}\right\rangle_{\mathrm{o \mu e}}^{1 / 2}
$$

The $\left\langle r^{2}\right\rangle_{\mathrm{o} \mu \mathrm{e}}^{1 / 2}$ are the results of a least-squares adjustment of the $\delta\left\langle r^{2}\right\rangle_{\text {oue }}$ taken from Table X. The $\left\langle r^{2}\right\rangle_{\mu \mathrm{e}}^{1 / 2}$ are obtained directly from the $R_{k \alpha}^{\mu}$ listed in Tables IIIA and IIIC with the help of the $V_{2}$ factors from Table X (see Eq. (5.6)). Radii for radioactive isotopes of lead are from a separate analysis for the nonprojected isotope shifts given in Table I. 
TABLE I. Observed (Projected) Optical Isotope Shifts of Selected Elements See page 194 for Explanation of Tables

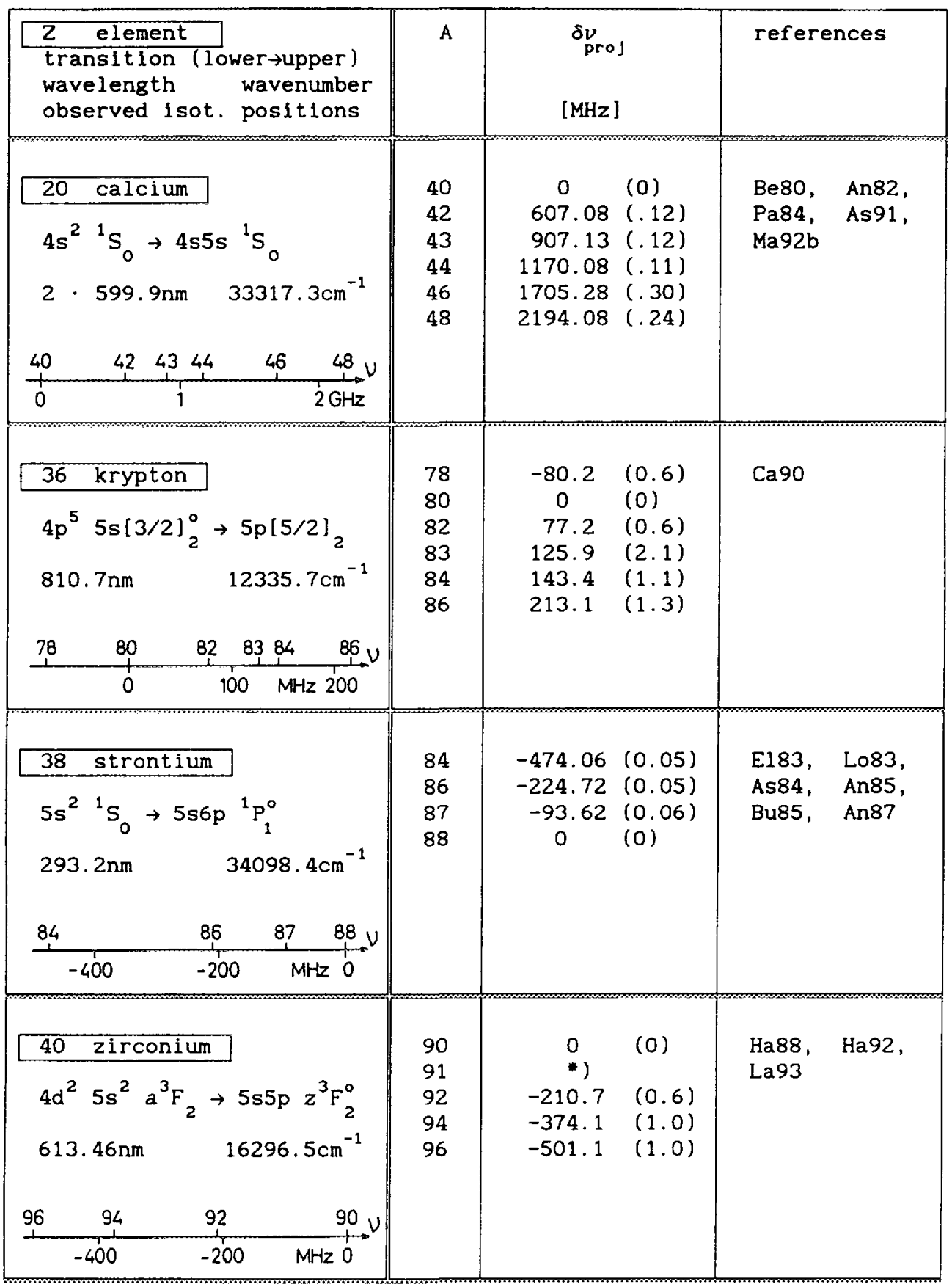

-) The optical measurements for this isotope heavily disagree 
TABLE I. Observed (Projected) Optical Isotope Shifts of Selected Elements See page 194 for Explanation of Tables

\begin{tabular}{|c|c|c|c|c|c|}
\hline 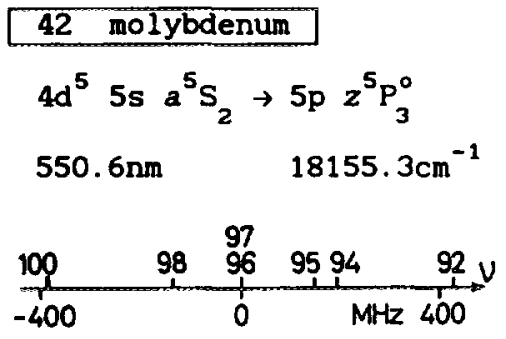 & $\begin{array}{l}92 \\
94 \\
95 \\
96 \\
97 \\
98 \\
100\end{array}$ & $\begin{array}{c}419.7 \\
188.9 \\
146.9 \\
0 \\
0.0 \\
-134.9 \\
-374.7\end{array}$ & $\begin{array}{l}(6.0) \\
(3.0) \\
(3.0) \\
(0) \\
(3.0) \\
(3.0) \\
(6.0)\end{array}$ & $\begin{array}{l}\text { Au78, } \\
0186\end{array}$ & Br84, \\
\hline 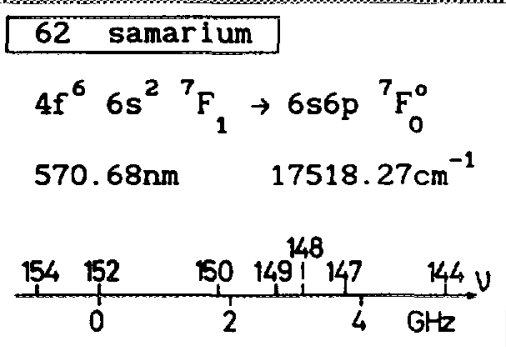 & $\begin{array}{l}144 \\
147 \\
148 \\
149 \\
150 \\
152 \\
154\end{array}$ & $\begin{array}{c}5322.6 \\
3749.4 \\
3097.2 \\
2697.0 \\
1801.1 \\
0 \\
-990.6\end{array}$ & $\begin{array}{l}(1.3) \\
(0.8) \\
(0.8) \\
(0.8) \\
(0.8) \\
(0) \\
(1.1)\end{array}$ & $\begin{array}{l}\text { Ha } 67, \\
\text { Br } 80, \\
\text { Wa } 90\end{array}$ & $\begin{array}{l}\text { Br79, } \\
\text { En90, }\end{array}$ \\
\hline 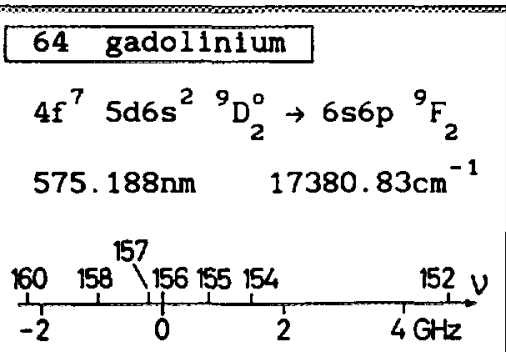 & $\begin{array}{l}154 \\
155 \\
156 \\
157 \\
158 \\
160\end{array}$ & $\begin{array}{r}1442.8 \\
737.4 \\
0 \\
-205.3 \\
-1079.7 \\
-2200.0\end{array}$ & $\begin{array}{l}(0.6) \\
(0.4) \\
(0) \\
(0.4) \\
(0.4) \\
(0.3)\end{array}$ & $\begin{array}{l}\text { Kr85, } \\
\text { Ji90, }\end{array}$ & $\begin{array}{l}\text { Du90, } \\
\text { Kr90 }\end{array}$ \\
\hline $\begin{array}{l}\frac{82 \text { lead }}{6 \mathrm{p}^{2}{ }^{3} \mathrm{P}_{\mathrm{o}} \rightarrow 6 \mathrm{p} 7 \mathrm{~s}^{3} \mathrm{P}_{1}^{0}} \\
283.3 \mathrm{~nm} \\
35287.2 \mathrm{~cm}^{-1}\end{array}$ & $\begin{array}{l}196 \\
197 \\
198 \\
199 \\
200 \\
201 \\
202 \\
203 \\
204^{\star} \\
205 \\
206^{*} \\
207^{\star} \\
208^{\star} \\
209 \\
210 \\
211 \\
212 \\
214\end{array}$ & $\begin{array}{c}-11441 \\
-11402 \\
-9848 \\
-9748 \\
-8094.1 \\
-7727.6 \\
-6193.7 \\
-5749.0 \\
-4211.8 \\
-3712.6 \\
-2227.1 \\
-1390.9 \\
0 \\
1767 \\
3973.7 \\
5648.2 \\
7815 \\
11503\end{array}$ & $\begin{array}{l}(30) \\
(19) \\
(3.5) \\
(9) \\
(3.5) \\
(5.0) \\
(3.5) \\
(5.0) \\
(2.5) \\
(3.0) \\
(2.5) \\
(2.5) \\
(0) \\
(9) \\
(3.5) \\
(5.6) \\
(30) \\
(20)\end{array}$ & $\begin{array}{l}\text { St52, } \\
\text { Th83, } \\
\text { Bu88 }\end{array}$ & $\begin{array}{l}\text { B158, } \\
\text { An86, }\end{array}$ \\
\hline
\end{tabular}

*) stable isotopes, projected values. From the investigated lighter radioactive isotopes (190 through 203) only those are given here, where the line 283.3nm was investigated. The shifts for the radioactive isotopes were not projected, but their values for the stable isotopes agree well with the projected values. 
TABLE II. Observed $K \alpha$ X-Ray Isotope Shifts $\delta E$ and Charge Radius Variations $\lambda$ See page 194 for Explanation of Tables

\begin{tabular}{|c|c|c|c|}
\hline Isotope Pair & $\begin{array}{c}\delta E_{C o u l .}^{A A^{\prime}} \\
{[\mathrm{meV}]}\end{array}$ & $\begin{array}{c}-\lambda^{A A^{\prime}} \\
{\left[\mathrm{fm}^{2}\right]}\end{array}$ & Ref. \\
\hline${ }^{92-100} \mathrm{Mo}$ & $35.0 \pm 5.0$ & $1.03 \pm 0.15$ & [Se69] \\
\hline${ }^{94-100} \mathrm{Mo}$ & $31.0 \pm 8.0$ & $0.91 \pm 0.23$ & [Se69] \\
\hline${ }^{116-124} S n$ & $35.0 \pm 1.3$ & $0.414 \pm 0.016$ & [Ch68] \\
\hline${ }^{121-123} S b$ & $1.8 \pm 1.0$ & $0.02 \pm 0.01$ & $\{R y 72]$ \\
\hline${ }^{124-128} \mathrm{Te}$ & $8.4 \pm 2.4$ & $0.081 \pm 0.023$ & [Su69] \\
\hline${ }^{128-128} \mathrm{Te}$ & $5.7 \pm 2.4$ & $0.055 \pm 0.023$ & [Su69] \\
\hline${ }^{128-130} \mathrm{Te}$ & $4.9 \pm 2.4$ & $0.047 \pm 0.023$ & [Su69] \\
\hline${ }^{134-135} B a$ & $-6.3 \pm 2.0$ & $-0.040 \pm 0.013$ & {$[\mathrm{Ha} 67]$} \\
\hline${ }^{134-136} \mathrm{Ba}$ & $-3.4 \pm 2.0$ & $-0.022 \pm 0.013$ & [Ha67] \\
\hline${ }^{136-137} B a$ & $-0.2 \pm 2.0$ & $-0.001 \pm 0.013$ & [Ha67] \\
\hline${ }^{138-138} B a$ & $9.0 \pm 2.0$ & $0.057 \pm 0.013$ & {$[\mathrm{Ha} 67]$} \\
\hline${ }^{140-142} \mathrm{Ce}$ & $51.5 \pm 1.9$ & $0.274 \pm 0.010$ & [Ei70a] \\
\hline${ }^{142-143} N d$ & $27.7 \pm 5.1$ & $0.116 \pm 0.021$ & [Le73] \\
\hline${ }^{142-144} N d$ & $68.7 \pm 2.6$ & $0.289 \pm 0.011$ & [Le73] \\
\hline${ }^{143-144} N d$ & $41.0 \pm 3.6$ & $0.172 \pm 0.015$ & [Le73] \\
\hline${ }^{144-145} N d$ & $29.8 \pm 4.2$ & $0.126 \pm 0.018$ & [Le73] \\
\hline \multirow[t]{2}{*}{${ }^{144-146} N d$} & $55.4 \pm 7.7$ & $0.233 \pm 0.033$ & [Bh69],[Le73] \\
\hline & $64.0 \pm 11.0$ & $0.269 \pm 0.046$ & [Su67] \\
\hline${ }^{145-146} N d$ & $25.5 \pm 8.8$ & $0.107 \pm 0.037$ & [Bh69],[Le73] \\
\hline \multirow[t]{2}{*}{${ }^{146-148} N d$} & $65.4 \pm 8.0$ & $0.275 \pm 0.034$ & {$[$ Bh69],[Le73] } \\
\hline & $66.0 \pm 10.0$ & $0.277 \pm 0.042$ & [Su67] \\
\hline \multirow[t]{2}{*}{${ }^{148-150} N d$} & $95.8 \pm 5.0$ & $0.403 \pm 0.021$ & [Bh69],[Le73] \\
\hline & $110.0 \pm 13.0$ & $0.466 \pm 0.055$ & [Su67] \\
\hline${ }^{144-148} \mathrm{Sm}$ & $139.0 \pm 24.0$ & $0.478 \pm 0.083$ & [Su67] \\
\hline${ }^{147-148} \mathrm{Sm}$ & $49.8 \pm 2.9$ & $0.171 \pm 0.010$ & [Le73] \\
\hline${ }^{148-149} \mathrm{Sm}$ & $23.2 \pm 2.3$ & $0.080 \pm 0.008$ & [Le73] \\
\hline \multirow[t]{2}{*}{${ }^{148-150} \mathrm{Sm}$} & $88.3 \pm 3.0$ & $0.303 \pm 0.010$ & [Le73] \\
\hline & $102.0 \pm 15.0$ & $0.350 \pm 0.051$ & [Su67] \\
\hline${ }^{149-150} \mathrm{Sm}$ & $65.1 \pm 3.0$ & $0.224 \pm 0.010$ & [Le73] \\
\hline \multirow[t]{2}{*}{${ }^{150-152} \mathrm{Sm}$} & $119.5 \pm 3.5$ & $0.411 \pm 0.012$ & [Le73] \\
\hline & $110.0 \pm 16.0$ & $0.378 \pm 0.055$ & [Su67] \\
\hline${ }^{152-154} \mathrm{Sm}$ & $64.3 \pm 3.8$ & $0.221 \pm 0.013$ & [Le73] \\
\hline${ }^{151-153} E u$ & $186.0 \pm 10.0$ & $0.581 \pm 0.031$ & {$[R y 72]$} \\
\hline${ }^{154-150} G d$ & $39.2 \pm 8.1$ & $0.112 \pm 0.024$ & [Bh69] \\
\hline${ }^{154-156} G d$ & $71.4 \pm 8.1$ & $0.203 \pm 0.023$ & [Bh69] \\
\hline${ }^{155-156} \mathrm{Gd}$ & $32.2 \pm 3.6$ & $0.092 \pm 0.013$ & [Bh69] \\
\hline${ }^{156-157} G d$ & $10.4 \pm 4.4$ & $0.030 \pm 0.013$ & [Bh69] \\
\hline
\end{tabular}


G. FRICKE et al. Nuclear Charge Radii

TABLE Il. Observed $K \alpha$ X-Ray Isotope Shifts $\delta E$ and Charge Radius Variations $\lambda$ See page 194 for Explanation of Tables

\begin{tabular}{|c|c|c|c|}
\hline Isotope Pair & $\begin{array}{c}\delta E_{\text {Coull. }}^{A^{\prime}} \\
{[\mathrm{meV}]}\end{array}$ & $\begin{array}{c}-\lambda^{A A^{\prime}} \\
{\left[\mathrm{fm}^{2}\right]}\end{array}$ & Ref. \\
\hline${ }^{156-158} \mathrm{Gd}$ & $50.6 \pm 3.6$ & $0.144 \pm 0.010$ & [Bh69] \\
\hline${ }^{157-158} G d$ & $40.6 \pm 3.7$ & $0.116 \pm 0.011$ & [Bh69] \\
\hline${ }^{188-180} G d$ & $54.0 \pm 3.4$ & $0.154 \pm 0.010$ & [Bh69] \\
\hline${ }^{161-162} D y$ & $39.4 \pm 5.1$ & $0.092 \pm 0.012$ & [Le73] \\
\hline${ }^{162-163} D y$ & $4.2 \pm 3.3$ & $0.010 \pm 0.008$ & [Le73] \\
\hline \multirow[t]{2}{*}{${ }^{162-164} D y$} & $55.6 \pm 3.7$ & $0.130 \pm 0.009$ & [Le73] \\
\hline & $58.1 \pm 3.3$ & $0.136 \pm 0.008$ & [Ei70b] \\
\hline${ }^{163-164} D y$ & $51.4 \pm 4.7$ & $0.120 \pm 0.011$ & [Le73] \\
\hline${ }^{166-168} \mathrm{Er}$ & $69.5 \pm 4.5$ & $0.135 \pm 0.009$ & [Bh69] \\
\hline${ }^{168-170} \mathrm{Er}$ & $80.0 \pm 6.1$ & $0.155 \pm 0.012$ & [Bh69] \\
\hline${ }^{170-171} Y b$ & $48.0 \pm 20.2$ & $0.077 \pm 0.032$ & [Le73] \\
\hline${ }^{170-172} Y b$ & $101.4 \pm 11.6$ & $0.163 \pm 0.019$ & [Le73] \\
\hline $171-172 Y b$ & $53.4 \pm 8.6$ & $0.086 \pm 0.014$ & [Le73] \\
\hline${ }^{172-173} Y b$ & $31.2 \pm 16.9$ & $0.050 \pm 0.027$ & [Le73] \\
\hline${ }^{172-174} Y b$ & $88.0 \pm 8.4$ & $0.141 \pm 0.013$ & [Le73] \\
\hline $173-174 Y b$ & $56.8 \pm 20.6$ & $0.091 \pm 0.033$ & [Le73] \\
\hline${ }^{174-17 \theta} Y b$ & $65.5 \pm 7.4$ & $0.103 \pm 0.012$ & [Le73] \\
\hline${ }^{178-180} \mathrm{Hf}$ & $77.4 \pm 5.3$ & $0.103 \pm 0.007$ & {$[\mathrm{Bh} 69]$} \\
\hline${ }^{182-184} W$ & $92.3 \pm 10.5$ & $0.102 \pm 0.012$ & [Ch68] \\
\hline${ }^{184-186} W$ & $60.0 \pm 8.0$ & $0.066 \pm 0.009$ & [Ch68] \\
\hline${ }^{200-204} \mathrm{Hg}$ & $254.0 \pm 37.0$ & $0.162 \pm 0.024$ & [Ch68] \\
\hline \multirow[t]{2}{*}{${ }^{204-206} \mathrm{~Pb}$} & $200.0 \pm 38.0$ & $0.106 \pm 0.020$ & [Le73] \\
\hline & $186.0 \pm 20.0$ & $0.099 \pm 0.011$ & [Bo83] \\
\hline \multirow[t]{3}{*}{${ }^{204-208} \mathrm{~Pb}$} & $386.0 \pm 42.0$ & $0.205 \pm 0.022$ & [Le73] \\
\hline & $414.0 \pm 17.0$ & $0.220 \pm 0.009$ & [Bo83] \\
\hline & & $0.2175 \pm 0.0085^{\star}$ & [Bo83] \\
\hline \multirow[t]{2}{*}{${ }^{206-207} \mathrm{~Pb}$} & $50.0 \pm 20.0$ & $0.027 \pm 0.011$ & [Le73] \\
\hline & $78.0 \pm 14.0$ & $0.041 \pm 0.010$ & [Bo83] \\
\hline \multirow[t]{3}{*}{${ }^{200-208} P b$} & $186.0 \pm 18.0$ & $0.099 \pm 0.010$ & [Ch68],[Le73] \\
\hline & $228.0 \pm 14.0$ & $0.121 \pm 0.007$ & [Bo83] \\
\hline & & $0.113 \pm 0.008^{\star}$ & [Bo83] \\
\hline \multirow[t]{3}{*}{${ }^{207-208} P b$} & $136.0 \pm 25.0$ & $0.072 \pm 0.013$ & [Le73] \\
\hline & $150.0 \pm 14.0$ & $0.080 \pm 0.007$ & [Bo83] \\
\hline & & $0.0780 \pm 0.0065^{\star}$ & {$[\mathrm{Bo} 83]$} \\
\hline${ }^{235}-238 \mathrm{U}$ & $1800 \pm 200$ & $0.383 \pm 0.044$ & {$[\mathrm{Br} 65]$} \\
\hline
\end{tabular}

* Weighted average of the data-sets from [Le73] and [Bo83] 
TABLE IIIA. Muonic $2 p \rightarrow 1 s$ Transition Energies and Barrett Radii for $Z<60$ and $Z>77$ See page 194 for Explanation of Tables

\begin{tabular}{|c|c|c|c|c|c|c|c|c|c|c|}
\hline Isotope & $\begin{array}{l}E_{\text {exp. }} \\
{[\mathrm{keV}]}\end{array}$ & $\begin{array}{l}E_{\text {theo. }} \\
{[\mathrm{keV}]}\end{array}$ & $\begin{array}{l}\text { NPol } \\
{[\mathrm{keV}]}\end{array}$ & $\begin{array}{c}\mathbf{c} \\
{[\mathbf{f m}]}\end{array}$ & $\begin{array}{c}\left\langle r^{2}\right\rangle_{\text {model }}^{\mathbf{1 / 2}} \\
{[\mathbf{f m}]}\end{array}$ & $\begin{array}{c}\alpha \\
{[1 / \text { fm }]}\end{array}$ & $\mathbf{k}$ & $\begin{array}{c}C_{\mathrm{x}} \\
{[\mathrm{am} / \mathrm{eV}]}\end{array}$ & $\begin{array}{l}R_{h \alpha}^{\mu} \\
{[\mathbf{f m}]}\end{array}$ & Ref. \\
\hline${ }^{9} B e^{\dagger}$ & $\begin{array}{r}33.402 \\
10\end{array}$ & 33.402 & 0.001 & $\begin{array}{r}1.7890 \\
3700\end{array}$ & 2.390 & 0.0420 & 2.1160 & -20.80 & $\begin{array}{c}3.0725 \\
(2080 ; 60)\end{array}$ & [Sc80a] \\
\hline${ }_{\text {nat }} B^{\dagger}$ & $\begin{array}{r}52.257 \\
7\end{array}$ & 52.262 & 0.001 & $\begin{array}{r}1.9280 \\
900\end{array}$ & 2.452 & 0.0440 & 2.1190 & -8.600 & $\begin{array}{c}3.1549 \\
(602 ; 30)\end{array}$ & [Sc80a] \\
\hline${ }^{12} C$ & $\begin{array}{r}75.2582 \\
5\end{array}$ & 75.2582 & 0.0025 & $\begin{array}{r}2.0005 \\
23\end{array}$ & 2.468 & 0.0208 & 2.0231 & -4.141 & $\begin{array}{l}3.1996 \\
(21 ; 33)\end{array}$ & $\begin{array}{c}{[\mathrm{Ru} 84 \mathrm{a}]} \\
{[\mathrm{Sc} 82]}\end{array}$ \\
\hline${ }^{13} C^{\ddagger}$ & $\begin{array}{r}75.3127 \\
40\end{array}$ & 75.3127 & 0.0025 & $\begin{array}{r}1.9958 \\
187\end{array}$ & 2.466 & 0.0208 & 2.0231 & -4.135 & $\begin{array}{c}3.1967 \\
(165 ; 31)\end{array}$ & $\begin{array}{c}{[\mathrm{Sc8} 8]} \\
{[\mathrm{Ru} 84 \mathrm{a}]}\end{array}$ \\
\hline${ }^{14} C^{\ddagger}$ & $\begin{array}{r}75.3514 \\
30\end{array}$ & 75.3514 & 0.0025 & $\begin{array}{r}2.0445 \\
137\end{array}$ & 2.492 & 0.0208 & 2.0234 & -4.095 & $\begin{array}{c}3.2273 \\
(123 ; 29)\end{array}$ & $\begin{array}{c}\text { [Sc82] } \\
{[\mathrm{Ru} 84 \mathrm{a}]}\end{array}$ \\
\hline${ }_{\text {nat }} N^{\dagger}$ & $\begin{array}{r}102.403 \\
5\end{array}$ & 102.404 & 0.003 & $\begin{array}{r}2.1510 \\
230\end{array}$ & 2.560 & 0.0470 & 2.1120 & -2.200 & $\begin{array}{c}3.2921 \\
(110 ; 20)\end{array}$ & [Sc80a] \\
\hline${ }^{16} \mathrm{O}$ & $\begin{array}{r}133.535 \\
2\end{array}$ & 133.534 & 0.005 & $\begin{array}{r}2.4130 \\
26\end{array}$ & 2.693 & 0.0272 & 2.0330 & -1.287 & $\begin{array}{l}3.4694 \\
(26 ; 22)\end{array}$ & [Fr92] \\
\hline${ }^{18} \mathrm{O}$ & $\begin{array}{r}133.572 \\
9\end{array}$ & 133.572 & 0.005 & $\begin{array}{r}2.5540 \\
130\end{array}$ & 3.586 & 0.0258 & 2.0287 & -1.258 & $\begin{array}{c}3.5680 \\
(113 ; 21)\end{array}$ & [Fr92] \\
\hline${ }^{19} \mathrm{~F}$ & $\begin{array}{r}168.515 \\
2\end{array}$ & 168.515 & 0.009 & $\begin{array}{r}2.7759 \\
15\end{array}$ & 2.898 & 0.0300 & 2.0392 & -0.782 & $\begin{array}{l}3.7291 \\
(16 ; 24)\end{array}$ & [Fr92] \\
\hline${ }^{20} \mathrm{Ne}$ & $\begin{array}{r}207.282 \\
5\end{array}$ & 207.282 & 0.019 & $\begin{array}{r}2.9589 \\
24\end{array}$ & 3.006 & 0.0329 & 2.0445 & -0.516 & $\begin{array}{l}3.8656 \\
(26 ; 33)\end{array}$ & [Fr92] \\
\hline${ }^{21} \mathrm{Ne}$ & $\begin{array}{r}207.429 \\
4\end{array}$ & 207.430 & 0.018 & $\begin{array}{r}2.8941 \\
20\end{array}$ & 2.967 & 0.0330 & 2.0441 & -0.521 & $\begin{array}{l}3.8163 \\
(21 ; 31)\end{array}$ & [Fr92] \\
\hline${ }^{22} \mathrm{Ne}$ & $\begin{array}{r}207.512 \\
4\end{array}$ & 207.512 & 0.018 & $\begin{array}{r}2.8706 \\
11\end{array}$ & 2.954 & 0.0330 & 2.0439 & -0.522 & $\begin{array}{l}3.7986 \\
(21 ; 31)\end{array}$ & [Fr92] \\
\hline
\end{tabular}

$\dagger$ Here, all data are taken from the quoted reference

$\ddagger$ Given energy based on $\mathrm{E}\left({ }^{12} \mathrm{C}\right)$ [Ru84a] and [Sc82] 
TABLE IIIA. Muonic $2 p \rightarrow 1 s$ Transition Energies and Barrett Radii for $Z<60$ and $Z>77$ See page 194 for Explanation of Tables

\begin{tabular}{|c|c|c|c|c|c|c|c|c|c|c|}
\hline Isotope & $\begin{array}{c}E_{\text {exp. }} \\
{[\mathrm{keV}]}\end{array}$ & $\begin{array}{l}E_{\text {theo. }} \\
\text { [keV] }\end{array}$ & $\begin{array}{l}\text { NPol } \\
{[\mathrm{keV}]}\end{array}$ & $\begin{array}{c}c \\
{[\mathrm{fm}]}\end{array}$ & $\begin{array}{c}\left\langle r^{2}\right\rangle_{\text {model }}^{1 / 2} \\
{[\mathrm{fm}]}\end{array}$ & $\begin{array}{c}\alpha \\
{[1 / f \mathbf{m}]}\end{array}$ & $\mathbf{k}$ & $\begin{array}{c}C_{x} \\
{[\mathrm{am} / \mathrm{eV}]}\end{array}$ & $\begin{array}{l}R_{k a}^{\mu} \\
{[\mathrm{fm}]}\end{array}$ & Ref. \\
\hline${ }^{23} \mathrm{Na}$ & $\begin{array}{r}250.229 \\
2\end{array}$ & 250.229 & 0.025 & $\begin{array}{r}2.9393 \\
7\end{array}$ & 2.994 & 0.0360 & 2.0484 & -0.364 & $\begin{array}{l}3.8492 \\
(7 ; 30)\end{array}$ & [Fr 92$]$ \\
\hline${ }^{24} M g$ & $\begin{array}{r}296.534 \\
2\end{array}$ & 296.533 & 0.038 & $\begin{array}{r}3.0453 \\
5\end{array}$ & 3.057 & 0.0389 & 2.0533 & -0.262 & $\begin{array}{c}3.9291 \\
(5 ; 30)\end{array}$ & [F!92] \\
\hline${ }^{25} \mathrm{Mg}$ & $\begin{array}{r}296.721 \\
3\end{array}$ & 296.721 & 0.031 & $\begin{array}{r}2.9978 \\
7\end{array}$ & 3.029 & 0.0390 & 2.0529 & -0.264 & $\begin{array}{c}3.8924 \\
(8 ; 25)\end{array}$ & {$\left[F_{I} 92\right]$} \\
\hline${ }^{26} \mathrm{Mg}$ & $\begin{array}{r}296.745 \\
3\end{array}$ & 296.745 & 0.033 & $\begin{array}{r}3.0066 \\
7\end{array}$ & 3.034 & 0.0390 & 2.0530 & -0.263 & $\begin{array}{c}3.8992 \\
(8 ; 26)\end{array}$ & [Fr92] \\
\hline${ }^{27} \mathrm{Al}$ & $\begin{array}{r}346.828 \\
2\end{array}$ & 346.827 & 0.040 & $\begin{array}{r}3.0554 \\
4\end{array}$ & 3.063 & 0.0419 & 2.0573 & -0.196 & $\begin{array}{c}3.9354 \\
(4 ; 24)\end{array}$ & [Fr92] \\
\hline${ }^{28} S i$ & $\begin{array}{r}400.173 \\
5\end{array}$ & 400.173 & 0.055 & $\begin{array}{r}3.1544 \\
7\end{array}$ & 3.123 & 0.0446 & 2.0621 & -0.149 & $\begin{array}{c}4.0112 \\
(7 ; 25)\end{array}$ & {$[\mathrm{Fr} 92]$} \\
\hline${ }^{29} \mathrm{Si}^{\dagger}$ & $\begin{array}{r}400.375 \\
45\end{array}$ & 400.375 & 0.053 & $\begin{array}{r}3.1482 \\
86\end{array}$ & 3.120 & 0.0446 & 2.0620 & -0.149 & $\begin{array}{r}4.0060 \\
(67 ; 26)\end{array}$ & {$[\mathrm{Fr} 92]$} \\
\hline${ }^{30} \mathrm{Si}^{\dagger}$ & $\begin{array}{r}400.295 \\
44\end{array}$ & 400.295 & 0.051 & $\begin{array}{r}3.1720 \\
84\end{array}$ & 3.134 & 0.0446 & 2.0622 & -0.149 & $\begin{array}{c}4.0250 \\
(66 ; 26)\end{array}$ & [Fr92] \\
\hline${ }^{31} P$ & $\begin{array}{r}456.800 \\
11\end{array}$ & 456.803 & 0.061 & $\begin{array}{r}3.2646 \\
11\end{array}$ & 3.190 & 0.0473 & 2.0671 & -0.116 & $\begin{array}{l}4.0969 \\
(13 ; 21)\end{array}$ & [Sc85] \\
\hline${ }^{32} S$ & $\begin{array}{r}516.330 \\
12\end{array}$ & 516.329 & 0.083 & $\begin{array}{r}3.3816 \\
6\end{array}$ & 3.263 & 0.0498 & 2.0722 & -0.092 & $\begin{array}{l}4.1892 \\
(11 ; 23)\end{array}$ & [Sc85] \\
\hline${ }^{34} S$ & $\begin{array}{r}516.106 \\
14\end{array}$ & 516.109 & 0.079 & $\begin{array}{r}3.4175 \\
7\end{array}$ & 3.285 & 0.0497 & 2.0726 & -0.091 & $\begin{array}{c}4.2181 \\
(13 ; 22)\end{array}$ & [Sc85] \\
\hline${ }^{36} S$ & $\begin{array}{r}515.981 \\
13\end{array}$ & 515.981 & 0.055 & $\begin{array}{r}3.4411 \\
10\end{array}$ & 3.300 & 0.0497 & 2.0728 & -0.091 & $\begin{array}{l}4.2371 \\
(12 ; 15)\end{array}$ & [Sc85] \\
\hline
\end{tabular}

$\dagger$ Here, all data are taken from the quoted reference 
TABLE IIIA. Muonic $2 p \rightarrow 1 s$ Transition Energies and Barrett Radii for $Z<60$ and $Z>77$ See page 194 for Explanation of Tables

\begin{tabular}{|c|c|c|c|c|c|c|c|c|c|c|}
\hline Isotope & $\begin{array}{c}E_{\text {exp. }} \\
{[\mathrm{keV}]}\end{array}$ & $\begin{array}{l}E_{\text {theo. }} \\
\text { [keV] }\end{array}$ & $\begin{array}{l}\text { NPol } \\
{[\mathrm{keV}]}\end{array}$ & $\begin{array}{c}c \\
{[\mathrm{fm}]}\end{array}$ & $\begin{array}{c}\left\langle r^{2}\right\rangle_{\text {model }}^{1 / 2} \\
{[\mathrm{fm}]}\end{array}$ & $\begin{array}{c}\boldsymbol{\alpha} \\
{[1 / \mathrm{fm}]}\end{array}$ & $\mathbf{k}$ & $\begin{array}{c}C_{z} \\
{[\mathrm{am} / \mathrm{eV}]}\end{array}$ & $\begin{array}{l}R_{k \alpha \alpha}^{\mu} \\
{[\mathrm{fm}]}\end{array}$ & Ref. \\
\hline${ }^{36} \mathrm{Ar}$ & $\begin{array}{r}644.597 \\
24\end{array}$ & 644.597 & 0.118 & $\begin{array}{r}3.5845 \\
18\end{array}$ & 3.390 & 0.0548 & 2.0821 & -0.060 & $\begin{array}{l}4.3515 \\
(14 ; 21)\end{array}$ & [Fr82] \\
\hline${ }^{38} \mathrm{Ar}$ & $\begin{array}{r}644.434 \\
24\end{array}$ & 644.432 & 0.107 & $\begin{array}{r}3.6025 \\
17\end{array}$ & 3.402 & 0.0547 & 2.0822 & -0.060 & $\begin{array}{l}4.3664 \\
(14 ; 19)\end{array}$ & [Fr82] \\
\hline${ }^{40} \mathrm{Ar}$ & $\begin{array}{r}644.004 \\
25\end{array}$ & 644.000 & 0.126 & $\begin{array}{r}3.6416 \\
18\end{array}$ & 3.427 & 0.0546 & 2.0827 & -0.060 & $\begin{array}{l}4.3986 \\
(15 ; 23)\end{array}$ & [Fr82] \\
\hline${ }^{39} K^{\star}$ & $\begin{array}{r}713.118 \\
32\end{array}$ & 713.118 & 0.119 & $\begin{array}{r}3.6542 \\
19\end{array}$ & 3.435 & 0.0572 & 2.0866 & -0.050 & $\begin{array}{l}4.4077 \\
(16 ; 18)\end{array}$ & [Wo81] \\
\hline${ }^{41} K^{\star}$ & $\begin{array}{r}712.769 \\
28\end{array}$ & 712.769 & 0.132 & $\begin{array}{r}3.6815 \\
17\end{array}$ & 3.452 & 0.0571 & 2.0869 & -0.050 & $\begin{array}{l}4.4303 \\
(14 ; 20)\end{array}$ & {$[$ Wo81] } \\
\hline${ }^{40} \mathrm{Ca}^{\star}$ & $\begin{array}{r}784.180 \\
25\end{array}$ & 784.180 & 0.142 & $\begin{array}{r}3.7221 \\
13\end{array}$ & 3.478 & 0.0596 & 2.0911 & -0.042 & $\begin{array}{l}4.4628 \\
(11 ; 18)\end{array}$ & [Wos1] \\
\hline${ }^{42} \mathrm{Ca}^{\star}$ & $\begin{array}{r}783.369 \\
29\end{array}$ & 783.369 & 0.166 & $\begin{array}{r}3.7690 \\
15\end{array}$ & 3.508 & 0.0595 & 2.0917 & -0.042 & $\begin{array}{l}4.5018 \\
(12 ; 21)\end{array}$ & [Wo $8 \mathrm{l}]$ \\
\hline${ }^{43} \mathrm{Ca}^{\star}$ & $\begin{array}{r}78.3 .811 \\
27\end{array}$ & 783.811 & 0.145 & $\begin{array}{r}3.7477 \\
14\end{array}$ & 3.495 & 0.0595 & 2.0914 & -0.042 & $\begin{array}{l}4.4840 \\
(11 ; 18)\end{array}$ & [Wo81] \\
\hline${ }^{44} \mathrm{Ca}^{\star}$ & $\begin{array}{r}783.156 \\
26\end{array}$ & 783.156 & 0.175 & $\begin{array}{r}3.7843 \\
13\end{array}$ & 3.518 & 0.0594 & 2.0919 & -0.042 & $\begin{array}{l}4.5146 \\
(11 ; 22)\end{array}$ & [Wo81] \\
\hline${ }^{40} \mathrm{Ca}^{\star}$ & $\begin{array}{r}783.817 \\
107\end{array}$ & 783.817 & 0.156 & $\begin{array}{r}3.7537 \\
54\end{array}$ & 3.498 & 0.0595 & 2.0915 & -0.042 & $\begin{array}{l}4.4891 \\
(45 ; 20)\end{array}$ & [Wo81] \\
\hline${ }^{48} \mathrm{Ca}^{\star}$ & $\begin{array}{r}784.487 \\
26\end{array}$ & 784.487 & 0.153 & $\begin{array}{r}3.7231 \\
13\end{array}$ & 3.479 & 0.0596 & 2.0912 & -0.042 & $\begin{array}{l}4.4636 \\
(11 ; 19)\end{array}$ & [Wo81] \\
\hline${ }^{45} S c^{\star \star}$ & $\begin{array}{r}855.185 \\
41 \\
857.005 \\
41\end{array}$ & $\begin{array}{l}855.184 \\
857.005\end{array}$ & $\begin{array}{l}0.182 \\
0.203\end{array}$ & $\begin{array}{r}3.8279 \\
12\end{array}$ & 3.546 & $\begin{array}{l}0.0619 \\
0.0618\end{array}$ & $\begin{array}{l}2.0962 \\
2.0961\end{array}$ & $\begin{array}{l}-0.036 \\
-0.0: 36\end{array}$ & $\begin{array}{c}4.5499 \\
(15 ; 20) \\
4.5499 \\
(15 ; 22)\end{array}$ & [He86] \\
\hline
\end{tabular}

* The energy corresponds to the $2 p_{3 / 2} \rightarrow 1 s_{1 / 2}$ transition

** The first (second) energy value corresponds to the $2 p_{1 / 2} \rightarrow 1 s_{1 / 2}$

$\left(2 p_{3 / 2} \rightarrow 1 s_{1 / 2}\right)$ transition 
TABLE IIIA. Muonic $2 p \rightarrow 1 s$ Transition Energies and Barrett Radii for $Z<60$ and $Z>77$ See page 194 for Explanation of Tables

\begin{tabular}{|c|c|c|c|c|c|c|c|c|c|c|}
\hline Isotope & $\begin{array}{c}E_{\text {exp. }} \\
{[\mathrm{keV}]}\end{array}$ & $\begin{array}{l}E_{\text {theo. }} \\
{[\mathrm{keV}]}\end{array}$ & $\begin{array}{l}\text { NPol } \\
\text { [keV] }\end{array}$ & $\begin{array}{c}\mathrm{c} \\
{[\mathrm{fm}]}\end{array}$ & $\begin{array}{c}\left\langle r^{2}\right\rangle_{\text {model }}^{1 / 2} \\
{[\mathrm{fm}]}\end{array}$ & $\begin{array}{c}\alpha \\
{[1 / \mathrm{fm}]}\end{array}$ & $\mathbf{k}$ & $\begin{array}{c}C_{z} \\
{[\mathrm{am} / \mathrm{eV}]}\end{array}$ & $\begin{array}{l}R_{k \alpha}^{\mu} \\
{[\mathrm{fm}]}\end{array}$ & Ref. \\
\hline${ }^{48} \mathrm{Ti}^{\star}$ & $\begin{array}{r}931.944 \\
26\end{array}$ & 931.994 & 0.257 & $\begin{array}{r}3.9201 \\
9\end{array}$ & 3.606 & 0.0640 & 2.1009 & -0.031 & $\begin{array}{l}4.6263 \\
(8 ; 24)\end{array}$ & [Wo81] \\
\hline${ }^{47} T i^{*}$ & $\begin{array}{r}932.474 \\
25\end{array}$ & 932.474 & 0.252 & $\begin{array}{r}3.9039 \\
9\end{array}$ & 3.596 & 0.0641 & 2.1007 & -0.031 & $\begin{array}{l}4.6126 \\
(8 ; 23)\end{array}$ & [Wo81] \\
\hline${ }^{48} \mathrm{~T} i{ }^{\star}$ & $\begin{array}{r}932.652 \\
26\end{array}$ & 932.652 & 0.241 & $\begin{array}{r}3.8984 \\
9\end{array}$ & 3.592 & 0.0641 & 2.1007 & -0.031 & $\begin{array}{l}4.6079 \\
(8 ; 22)\end{array}$ & [Wo81] \\
\hline${ }^{40} \mathrm{Ti}^{\star}$ & $\begin{array}{r}933.426 \\
33\end{array}$ & 933.426 & 0.215 & $\begin{array}{r}3.8705 \\
12\end{array}$ & 3.574 & 0.0642 & 2.1003 & -0.031 & $\begin{array}{l}4.5845 \\
(10 ; 20)\end{array}$ & [Wo81] \\
\hline${ }^{50} T_{i}{ }^{*}$ & $\begin{array}{r}933.588 \\
26\end{array}$ & 933.588 & 0.216 & $\begin{array}{r}3.8659 \\
10\end{array}$ & 3.571 & 0.0642 & 2.1003 & -0.031 & $\begin{array}{l}4.5806 \\
(8 ; 20)\end{array}$ & [Wo81] \\
\hline${ }^{51} V^{\star}$ & $\begin{array}{r}1012.201 \\
26\end{array}$ & 1012.201 & 0.245 & $\begin{array}{r}3.9101 \\
8\end{array}$ & 3.600 & 0.0665 & 2.1044 & -0.027 & $\begin{array}{l}4.6166 \\
(7 ; 20)\end{array}$ & [Wo81] \\
\hline${ }^{50} \mathrm{Cr}^{\star}$ & $\begin{array}{r}1091.178 \\
27\end{array}$ & 1091.178 & 0.333 & $\begin{array}{r}4.0035 \\
7\end{array}$ & 3.661 & 0.0686 & 2.1092 & -0.023 & $\begin{array}{l}4.6946 \\
(6 ; 23)\end{array}$ & [Wo81] \\
\hline${ }^{52} \mathrm{Cr}^{\star}$ & $\begin{array}{r}1092.286 \\
21\end{array}$ & 1092.286 & 0.299 & $\begin{array}{r}3.9742 \\
6\end{array}$ & 3.645 & 0.0687 & 2.1088 & -0.023 & $\begin{array}{l}4.6697 \\
(5 ; 21)\end{array}$ & [Wo81] \\
\hline${ }^{53} \mathrm{Cr}^{\star}$ & $\begin{array}{r}1091.381 \\
25\end{array}$ & 1091.381 & 0.302 & $\begin{array}{r}4.0001 \\
7\end{array}$ & 3.659 & 0.0686 & 2.1092 & -0.023 & $\begin{array}{l}4.6917 \\
(6 ; 21)\end{array}$ & [Wo81] \\
\hline${ }^{54} \mathrm{Cr}^{\star}$ & $\begin{array}{r}1089.888 \\
31\end{array}$ & 1089.888 & 0.318 & $\begin{array}{r}4.0424 \\
9\end{array}$ & 3.686 & 0.0684 & 2.1097 & -0.023 & $\begin{array}{l}4.7277 \\
(7 ; 22)\end{array}$ & [Wo81] \\
\hline${ }^{\mathrm{BS}} M n^{\star}$ & $\begin{array}{r}1172.854 \\
34\end{array}$ & 1172.854 & 0.364 & $\begin{array}{r}4.0728 \\
8\end{array}$ & 3.706 & 0.0707 & 2.1136 & -0.021 & $\begin{array}{l}4.7525 \\
(7 ; 23)\end{array}$ & [Wo81] \\
\hline
\end{tabular}

* The energy corresponds to the $2 p_{3 / 2} \rightarrow 1 s_{1 / 2}$ transition. 
TABLE IIIA. Muonic $2 p \rightarrow 1 s$ Transition Energies and Barrett Radii for $Z<60$ and $Z>77$ See page 194 for Explanation of Tables

\begin{tabular}{|c|c|c|c|c|c|c|c|c|c|c|}
\hline Isotope & $\begin{array}{c}E_{\text {exp. }} \\
{[\mathrm{keV}]}\end{array}$ & $\begin{array}{l}E_{\text {theo }} \\
{[\mathrm{keV}]}\end{array}$ & $\begin{array}{l}\text { NPol } \\
{[\mathrm{keV}]}\end{array}$ & $\begin{array}{c}c \\
{[\mathrm{fm}]}\end{array}$ & $\begin{array}{c}\left\langle r^{2}\right\rangle_{\text {model }}^{1 / 2} \\
{[\mathrm{fm}]}\end{array}$ & $\begin{array}{c}\alpha \\
{[1 / \mathrm{fm}]}\end{array}$ & k & $\begin{array}{c}C_{z} \\
{[\mathrm{am} / \mathrm{keV}]}\end{array}$ & $\begin{array}{l}R_{k a}^{\mu} \\
{[\mathbf{f m}]}\end{array}$ & Ref. \\
\hline${ }^{54} \mathrm{Fe}$ & $\begin{array}{r}1255.849 \\
63 \\
1260.011 \\
48\end{array}$ & $\begin{array}{l}1255.849 \\
1260.011\end{array}$ & $\begin{array}{l}0.362 \\
0.362\end{array}$ & $\begin{array}{r}4.0546 \\
8\end{array}$ & 3.694 & $\begin{array}{l}0.0732 \\
0.0731\end{array}$ & $\begin{array}{l}2.1170 \\
2.1168\end{array}$ & $\begin{array}{l}-18.180 \\
-18.150\end{array}$ & $\begin{array}{c}4.7357 \\
(11 ; 20) \\
4.7358 \\
(9 ; 20)\end{array}$ & [Sh76] \\
\hline${ }^{86} \mathrm{Fe}$ & $\begin{array}{r}1252.919 \\
58 \\
1257.047 \\
44\end{array}$ & $\begin{array}{l}1252.901 \\
1257.057\end{array}$ & $\begin{array}{l}0.403 \\
0.403\end{array}$ & $\begin{array}{r}4.1198 \\
3\end{array}$ & 3.738 & $\begin{array}{l}0.0729 \\
0.0728\end{array}$ & $\begin{array}{l}2.1179 \\
2.1177\end{array}$ & $\begin{array}{l}-18.170 \\
-18.140\end{array}$ & $\begin{array}{l}4.7915 \\
(11 ; 22) \\
4.7915 \\
(8 ; 22)\end{array}$ & [Sh76] \\
\hline${ }^{87} \mathrm{Fe}$ & $\begin{array}{r}1251.823 \\
73 \\
1255.896 \\
56\end{array}$ & $\begin{array}{l}1251.771 \\
1255.926\end{array}$ & $\begin{array}{l}0.390 \\
0.391\end{array}$ & $\begin{array}{r}4.1442 \\
1\end{array}$ & 3.754 & $\begin{array}{l}0.0728 \\
0.0727\end{array}$ & $\begin{array}{l}2.1183 \\
2.1181\end{array}$ & $\begin{array}{l}-18.170 \\
-18.140\end{array}$ & $\begin{array}{l}4.8124 \\
(13 ; 21) \\
4.8125 \\
(10 ; 21)\end{array}$ & [Sh76] \\
\hline${ }^{58} \mathrm{Fe}$ & $\begin{array}{r}1250.381 \\
67 \\
1254.460 \\
54\end{array}$ & $\begin{array}{l}1250.336 \\
1254.489\end{array}$ & $\begin{array}{l}0.400 \\
0.401\end{array}$ & $\begin{array}{r}4.1755 \\
9\end{array}$ & 3.774 & $\begin{array}{l}0.0727 \\
0.0726\end{array}$ & $\begin{array}{l}2.1187 \\
2.1185\end{array}$ & $\begin{array}{l}-18.170 \\
-18.140\end{array}$ & $\begin{array}{l}4.8393 \\
(12 ; 22) \\
4.8393 \\
(10 ; 22)\end{array}$ & [Sh76] \\
\hline${ }^{59} \mathrm{Co}$ & $\begin{array}{r}1330.553 \\
65 \\
1341.500 \\
50\end{array}$ & $\begin{array}{l}1336.565 \\
1341.493\end{array}$ & $\begin{array}{l}0.320 \\
0.438\end{array}$ & $\begin{array}{r}4.1958 \\
8\end{array}$ & 3.788 & $\begin{array}{l}0.0749 \\
0.0748\end{array}$ & $\begin{array}{l}2.1224 \\
2.1222\end{array}$ & $\begin{array}{l}-16.210 \\
-16.170\end{array}$ & $\begin{array}{l}4.8556 \\
(11 ; 16) \\
4.8557 \\
(8 ; 21)\end{array}$ & {$[\operatorname{Sh} 76]$} \\
\hline${ }^{50} \mathrm{Ni}$ & $\begin{array}{r}1427.112 \\
60 \\
1432.534 \\
46\end{array}$ & $\begin{array}{l}1427.033 \\
1432.580\end{array}$ & $\begin{array}{l}0.436 \\
0.437\end{array}$ & $\begin{array}{r}4.1772 \\
6\end{array}$ & 3.776 & $\begin{array}{l}0.0772 \\
0.0771\end{array}$ & $\begin{array}{l}2.1250 \\
2.1253\end{array}$ & $\begin{array}{l}-14.520 \\
-14.490\end{array}$ & $\begin{array}{l}4.8386 \\
(9 ; 19) \\
4.8386 \\
(7 ; 19)\end{array}$ & [Sh76] \\
\hline${ }^{60} \mathrm{Ni}$ & $\begin{array}{r}1423.860 \\
58 \\
1429.360 \\
45\end{array}$ & $\begin{array}{r}1423.835 \\
1429.375\end{array}$ & $\begin{array}{l}0.461 \\
0.461\end{array}$ & $\begin{array}{r}4.2328 \\
6\end{array}$ & 3.813 & $\begin{array}{l}0.0770 \\
0.0769\end{array}$ & $\begin{array}{l}2.1264 \\
2.1261\end{array}$ & $\begin{array}{l}-14.530 \\
-14.500\end{array}$ & $\begin{array}{l}4.8865 \\
(8 ; 20) \\
4.8865 \\
(7 ; 20)\end{array}$ & {$[\operatorname{Sh} 76]$} \\
\hline${ }^{01} \mathrm{Ni}$ & $\begin{array}{r}1422.849 \\
69 \\
1428.397 \\
54\end{array}$ & $\begin{array}{l}1422.832 \\
1428.408\end{array}$ & $\begin{array}{l}0.387 \\
0.426\end{array}$ & $\begin{array}{r}4.2490 \\
7\end{array}$ & 3.823 & $\begin{array}{l}0.0769 \\
0.0768\end{array}$ & $\begin{array}{l}2.1266 \\
2.1264\end{array}$ & $\begin{array}{l}-14.530 \\
-14.500\end{array}$ & $\begin{array}{l}4.9005 \\
(10 ; 17) \\
4.9005 \\
(8 ; 19)\end{array}$ & [Sh76] \\
\hline
\end{tabular}




\section{G. FRICKE et al. Nuclear Charge Radii}

TABLE IIIA. Muonic $2 p \rightarrow 1 s$ Transition Energies and Barrett Radii for $Z<60$ and $Z>77$ See page 194 for Explanation of Tables

\begin{tabular}{|c|c|c|c|c|c|c|c|c|c|c|}
\hline Isotope & $\begin{array}{l}E_{\text {exp. }} \\
{[\mathrm{keV}]}\end{array}$ & $\begin{array}{l}E_{\text {theo. }} \\
\text { [keV] }\end{array}$ & $\begin{array}{l}\text { NPol } \\
{[\mathrm{keV}]}\end{array}$ & $\begin{array}{c}c \\
{[\mathrm{fm}]}\end{array}$ & $\begin{array}{c}\left\langle r^{2}\right\rangle_{\text {model }}^{1 / 2} \\
{[\mathrm{fm}]}\end{array}$ & $\begin{array}{c}\alpha \\
{[1 / f \mathrm{fm}]}\end{array}$ & $k$ & $\begin{array}{c}C_{\mathrm{z}} \\
{[\mathrm{am} / \mathrm{keV}]}\end{array}$ & $\begin{array}{l}R_{k \alpha}^{\mu} \\
{[\mathrm{fm}]}\end{array}$ & Ref. \\
\hline${ }^{82} \mathrm{Ni}$ & $\begin{array}{r}1421.342 \\
59 \\
1426.814 \\
45\end{array}$ & $\begin{array}{l}1421.303 \\
1426.837\end{array}$ & $\begin{array}{l}0.457 \\
0.458\end{array}$ & $\begin{array}{r}4.2765 \\
6\end{array}$ & 3.842 & $\begin{array}{l}0.0768 \\
0.0767\end{array}$ & $\begin{array}{l}2.1270 \\
2.1268\end{array}$ & $\begin{array}{l}-14.540 \\
-14.510\end{array}$ & $\begin{array}{l}4.9242 \\
(9 ; 20) \\
4.9242 \\
(7 ; 20)\end{array}$ & [Sh76] \\
\hline${ }^{84} \mathrm{Ni}$ & $\begin{array}{r}1419.708 \\
63 \\
1425.226 \\
49\end{array}$ & $\begin{array}{l}1419.699 \\
1425.231\end{array}$ & $\begin{array}{l}0.436 \\
0.438\end{array}$ & $\begin{array}{r}4.3041 \\
7\end{array}$ & 3.860 & $\begin{array}{l}0.0767 \\
0.0766\end{array}$ & $\begin{array}{l}2.1274 \\
2.1271\end{array}$ & $\begin{array}{l}-14.540 \\
-14.510\end{array}$ & $\begin{array}{l}4.9481 \\
(9 ; 19) \\
4.9481 \\
(7 ; 19)\end{array}$ & [Sh76] \\
\hline${ }^{83} \mathrm{Cu}$ & $\begin{array}{r}1508.052 \\
60 \\
1514.452 \\
47\end{array}$ & $\begin{array}{l}1508.049 \\
1514.454\end{array}$ & $\begin{array}{l}0.467 \\
0.538\end{array}$ & $\begin{array}{r}4.3376 \\
6\end{array}$ & 3.883 & $\begin{array}{l}0.0788 \\
0.0786\end{array}$ & $\begin{array}{l}2.1312 \\
2.1310\end{array}$ & $\begin{array}{l}-13.120 \\
-13.080\end{array}$ & $\begin{array}{l}4.9761 \\
(8 ; 18) \\
4.9762 \\
(6 ; 21)\end{array}$ & [Sh76] \\
\hline${ }^{65} \mathrm{Cu}$ & $\begin{array}{r}1506.147 \\
62 \\
1512.534 \\
49\end{array}$ & $\begin{array}{l}1506.146 \\
1512.535\end{array}$ & $\begin{array}{l}0.428 \\
0.489\end{array}$ & $\begin{array}{r}4.3667 \\
6\end{array}$ & 3.902 & $\begin{array}{l}0.0786 \\
0.0785\end{array}$ & $\begin{array}{l}2.1317 \\
2.1314\end{array}$ & $\begin{array}{l}-13.120 \\
-13.090\end{array}$ & $\begin{array}{l}5.0014 \\
(8 ; 17) \\
5.0015 \\
(6 ; 19)\end{array}$ & [Sh76] \\
\hline${ }^{64} Z_{n}$ & $\begin{array}{r}1595.528 \\
59 \\
1602.709 \\
47\end{array}$ & $\begin{array}{l}1595.510 \\
1602.721\end{array}$ & $\begin{array}{l}0.608 \\
0.609\end{array}$ & $\begin{array}{r}4.4046 \\
5\end{array}$ & 3.928 & $\begin{array}{l}0.0806 \\
0.0805\end{array}$ & $\begin{array}{l}2.1355 \\
2.1352\end{array}$ & $\begin{array}{l}-11.890 \\
-11.860\end{array}$ & $\begin{array}{l}5.0333 \\
(7 ; 22) \\
5.0334 \\
(6 ; 22)\end{array}$ & [Sh76] \\
\hline${ }^{\circ} \mathrm{Zn}$ & $\begin{array}{r}1593.313 \\
61 \\
1600.553 \\
45\end{array}$ & $\begin{array}{l}1593.336 \\
1600.541\end{array}$ & $\begin{array}{l}0.594 \\
0.595\end{array}$ & $\begin{array}{r}4.4349 \\
5\end{array}$ & 3.948 & $\begin{array}{l}0.0805 \\
0.0803\end{array}$ & $\begin{array}{l}2.1360 \\
2.1357\end{array}$ & $\begin{array}{l}-11.900 \\
-11.860\end{array}$ & $\begin{array}{l}5.0598 \\
(7 ; 21) \\
5.0599 \\
(5 ; 21)\end{array}$ & [Sh76] \\
\hline${ }^{88} \mathrm{Zn}$ & $\begin{array}{r}1591.521 \\
38 \\
1598.800 \\
32\end{array}$ & $\begin{array}{l}1591.568 \\
1598.767\end{array}$ & $\begin{array}{l}0.580 \\
0.581\end{array}$ & $\begin{array}{r}4.4597 \\
3\end{array}$ & 3.965 & $\begin{array}{l}0.0804 \\
0.0802\end{array}$ & $\begin{array}{l}2.1364 \\
2.1361\end{array}$ & $\begin{array}{l}-11.910 \\
-11.870\end{array}$ & $\begin{array}{c}5.0814 \\
(5 ; 21) \\
5.0815 \\
(4 ; 21)\end{array}$ & [Sh76] \\
\hline${ }^{70} \mathrm{Zn}$ & $\begin{array}{r}1589.863 \\
180 \\
1596.817 \\
131\end{array}$ & $\begin{array}{l}1589.706 \\
1596.900\end{array}$ & $\begin{array}{l}0.614 \\
0.615\end{array}$ & $\begin{array}{r}4.4862 \\
14\end{array}$ & 3.983 & $\begin{array}{l}0.0803 \\
0.0801\end{array}$ & $\begin{array}{l}2.1368 \\
2.1364\end{array}$ & $\begin{array}{l}-11.920 \\
-11.880\end{array}$ & $\begin{array}{l}5.1046 \\
(21 ; 22) \\
5.1047 \\
(15 ; 22)\end{array}$ & [Sh76] \\
\hline
\end{tabular}


G. FRICKE et al. Nuclear Charge Radii

TABLE IIIA. Muonic $2 p \rightarrow 1 s$ Transition Energies and Barrett Radii for $Z<60$ and $Z>77$ See page 194 for Explanation of Tables

\begin{tabular}{|c|c|c|c|c|c|c|c|c|c|c|}
\hline Isotope & $\begin{array}{c}E_{\text {exp. }} \\
{[\mathrm{keV}]}\end{array}$ & $\begin{array}{l}E_{\text {theo }} \\
\text { [keV] }\end{array}$ & $\begin{array}{l}\text { NPol } \\
\text { [keV] }\end{array}$ & $\begin{array}{c}c \\
{[\mathrm{fm}]}\end{array}$ & $\begin{array}{c}\left\langle r^{2}\right\rangle_{\text {model }}^{1 / 2} \\
{[\mathrm{fm}]}\end{array}$ & $\begin{array}{c}\alpha \\
{[1 / \mathrm{fm}]}\end{array}$ & $\mathbf{k}$ & $\begin{array}{c}C_{x} \\
{[\mathrm{am} / \mathrm{keV}]}\end{array}$ & $\begin{array}{l}R_{k \alpha}^{\mu} \\
{[\mathrm{fm}]}\end{array}$ & Ref. \\
\hline${ }^{89} \mathrm{Ga}$ & $\begin{array}{r}1680.775 \\
14 \\
1689.036 \\
12\end{array}$ & $\begin{array}{l}1680.802 \\
1689.016\end{array}$ & $\begin{array}{l}0.512 \\
0.567\end{array}$ & $\begin{array}{r}4.5066 \\
1\end{array}$ & 3.996 & $\begin{array}{l}0.0823 \\
0.0821\end{array}$ & $\begin{array}{l}2.1404 \\
2.1400\end{array}$ & $\begin{array}{l}-10.840 \\
-10.810\end{array}$ & $\begin{array}{l}5.1214 \\
(2 ; 17) \\
5.1215 \\
(1 ; 18)\end{array}$ & [Ma83] \\
\hline${ }^{71} \mathrm{Ga}$ & $\begin{array}{r}1679.141 \\
14 \\
1687.331 \\
12\end{array}$ & $\begin{array}{l}1679.147 \\
1687.327\end{array}$ & $\begin{array}{l}0.526 \\
0.551\end{array}$ & $\begin{array}{r}4.5279 \\
1\end{array}$ & 4.011 & $\begin{array}{l}0.0822 \\
0.0820\end{array}$ & $\begin{array}{l}2.1407 \\
2.1403\end{array}$ & $\begin{array}{l}-10.850 \\
-10.820\end{array}$ & $\begin{array}{c}5.1401 \\
(2 ; 17) \\
5.1402 \\
(1 ; 18)\end{array}$ & [Ma83] \\
\hline${ }^{70} \mathrm{Ge}$ & $\begin{array}{r}1769.859 \\
23 \\
1779.070 \\
16\end{array}$ & $\begin{array}{l}1769.865 \\
1779.073\end{array}$ & $\begin{array}{l}0.704 \\
0.706\end{array}$ & $\begin{array}{r}4.5687 \\
2\end{array}$ & 4.039 & $\begin{array}{l}0.0841 \\
0.0839\end{array}$ & $\begin{array}{l}2.1446 \\
2.1442\end{array}$ & $\begin{array}{l}-9.925 \\
-9.891\end{array}$ & $\begin{array}{l}\mathbf{5 . 1 7 4 9} \\
(2 ; 21) \\
\mathbf{5 . 1 7 5 0} \\
(2 ; 21)\end{array}$ & [Si82a] \\
\hline${ }^{72} \mathrm{Ge}$ & $\begin{array}{r}1767.835 \\
17 \\
1777.043 \\
12\end{array}$ & $\begin{array}{l}1767.841 \\
1777.040\end{array}$ & $\begin{array}{l}0.735 \\
0.738\end{array}$ & $\begin{array}{r}4.5926 \\
2\end{array}$ & 4.055 & $\begin{array}{l}0.0840 \\
0.0838\end{array}$ & $\begin{array}{l}2.1449 \\
2.1445\end{array}$ & $\begin{array}{l}-9.936 \\
-9.902\end{array}$ & $\begin{array}{l}5.1959 \\
(2 ; 22) \\
5.1960 \\
(1 ; 22)\end{array}$ & [Si82a] \\
\hline${ }^{73} \mathrm{Ge}$ & $\begin{array}{r}1766.998 \\
34 \\
1776.244 \\
24\end{array}$ & $\begin{array}{l}1767.022 \\
1776.232\end{array}$ & $\begin{array}{l}0.682 \\
0.700\end{array}$ & $\begin{array}{r}4.6015 \\
2\end{array}$ & 4.061 & $\begin{array}{l}0.0840 \\
0.0837\end{array}$ & $\begin{array}{l}2.1451 \\
2.1447\end{array}$ & $\begin{array}{l}-9.940 \\
-9.899\end{array}$ & $\begin{array}{l}5.2038 \\
(3 ; 20) \\
5.2039 \\
(2 ; 21)\end{array}$ & [Si82a] \\
\hline${ }^{74} \mathrm{Ge}$ & $\begin{array}{r}1765.729 \\
26 \\
1774.881 \\
17\end{array}$ & $\begin{array}{l}1765.702 \\
1774.892\end{array}$ & $\begin{array}{l}0.836 \\
0.839\end{array}$ & $\begin{array}{r}4.6185 \\
2\end{array}$ & 4.072 & $\begin{array}{l}0.0839 \\
0.0837\end{array}$ & $\begin{array}{l}2.1453 \\
2.1449\end{array}$ & $\begin{array}{l}-9.947 \\
-9.912\end{array}$ & $\begin{array}{l}5.2187 \\
(3 ; 25) \\
5.2188 \\
(2 ; 25)\end{array}$ & [Si82a] \\
\hline${ }^{70} \mathrm{Ge}$ & $\begin{array}{r}1764.778 \\
23 \\
1773.946 \\
15\end{array}$ & $\begin{array}{l}1764.765 \\
1773.951\end{array}$ & $\begin{array}{l}0.817 \\
0.819\end{array}$ & $\begin{array}{r}4.6294 \\
1\end{array}$ & 4.080 & $\begin{array}{l}0.0838 \\
0.0836\end{array}$ & $\begin{array}{l}2.1455 \\
2.1451\end{array}$ & $\begin{array}{l}-9.946 \\
-9.912\end{array}$ & $\begin{array}{l}5.2283 \\
(2 ; 24) \\
5.2284 \\
(2 ; 24)\end{array}$ & [Si82a] \\
\hline${ }^{75} \mathrm{As}$ & $\begin{array}{r}1857.124 \\
12 \\
1867.664 \\
10\end{array}$ & $\begin{array}{l}1857.130 \\
1867.660\end{array}$ & $\begin{array}{l}0.557 \\
0.761\end{array}$ & $\begin{array}{r}4.6527 \\
1\end{array}$ & 4.096 & $\begin{array}{l}0.0858 \\
0.0855\end{array}$ & $\begin{array}{l}2.1491 \\
2.1486\end{array}$ & $\begin{array}{l}-9.132 \\
-9.094\end{array}$ & $\begin{array}{l}5.2479 \\
(1 ; 15) \\
5.2480 \\
(1 ; 21)\end{array}$ & [Ma83] \\
\hline
\end{tabular}


TABLE IIIA. Muonic $2 p \rightarrow 1 s$ Transition Energies and Barrett Radii for $Z<60$ and $Z>77$ See page 194 for Explanation of Tables

\begin{tabular}{|c|c|c|c|c|c|c|c|c|c|c|}
\hline Isotope & $\begin{array}{l}E_{\text {exp. }} \\
{[\mathrm{keV}]}\end{array}$ & $\begin{array}{l}E_{\text {theo. }} \\
{[\mathrm{keV}]}\end{array}$ & $\begin{array}{l}\text { NPol } \\
{[\mathrm{keV}]}\end{array}$ & $\begin{array}{c}\mathrm{c} \\
{[\mathrm{fm}]}\end{array}$ & $\begin{array}{c}\left\langle r^{2}\right\rangle_{\text {model }}^{1 / 2} \\
{[\mathrm{fm}]}\end{array}$ & $\begin{array}{c}\alpha \\
{[1 / f \mathrm{~m}]}\end{array}$ & $\mathbf{k}$ & $\begin{array}{c}C_{k} \\
{[\mathrm{am} / \mathrm{keV}]}\end{array}$ & $\begin{array}{l}R_{k a}^{\mu} \\
{[\mathrm{fm}]}\end{array}$ & Ref. \\
\hline${ }^{76} \mathrm{Se}$ & $\begin{array}{r}1947.121 \\
21 \\
1958.620 \\
16\end{array}$ & $\begin{array}{l}1947.086 \\
1958.641\end{array}$ & $\begin{array}{l}1.028 \\
1.036\end{array}$ & $\begin{array}{r}4.7162 \\
1\end{array}$ & 4.139 & $\begin{array}{l}0.0875 \\
0.0872\end{array}$ & $\begin{array}{l}2.1532 \\
2.1528\end{array}$ & $\begin{array}{l}-8.428 \\
-8.388\end{array}$ & $\begin{array}{c}5.3031 \\
(2 ; 26) \\
5.3032 \\
(1 ; 26)\end{array}$ & [Si82b] \\
\hline${ }^{77} \mathrm{Se}$ & $\begin{array}{r}1946.854 \\
20 \\
1958.417 \\
16\end{array}$ & $\begin{array}{l}1946.860 \\
1958.413\end{array}$ & $\begin{array}{l}0.784 \\
0.790\end{array}$ & $\begin{array}{r}4.7163 \\
1\end{array}$ & 4.139 & $\begin{array}{l}0.0875 \\
0.0872\end{array}$ & $\begin{array}{l}2.1532 \\
2.1528\end{array}$ & $\begin{array}{l}-8.428 \\
-8.388\end{array}$ & $\begin{array}{c}5.3031 \\
(2 ; 20) \\
5.3033 \\
(1 ; 20)\end{array}$ & [Si82b] \\
\hline${ }^{70} \mathrm{Se}$ & $\begin{array}{r}1946.852 \\
17 \\
1958.366 \\
13\end{array}$ & $\begin{array}{l}1946.826 \\
1958.381\end{array}$ & $\begin{array}{l}0.941 \\
0.949\end{array}$ & $\begin{array}{r}4.7184 \\
1\end{array}$ & 4.140 & $\begin{array}{l}0.0875 \\
0.0872\end{array}$ & $\begin{array}{l}2.1533 \\
2.1528\end{array}$ & $\begin{array}{l}-8.428 \\
-8.390\end{array}$ & $\begin{array}{l}5.3049 \\
(1 ; 24) \\
5.3051 \\
(1 ; 24)\end{array}$ & [Si82b] \\
\hline${ }^{80} \mathrm{Se}$ & $\begin{array}{r}1946.859 \\
14 \\
1958.404 \\
12\end{array}$ & $\begin{array}{l}1946.854 \\
1958.407\end{array}$ & $\begin{array}{l}0.860 \\
0.872\end{array}$ & $\begin{array}{r}4.7178 \\
1\end{array}$ & 4.140 & $\begin{array}{l}0.0875 \\
0.0872\end{array}$ & $\begin{array}{l}2.1533 \\
2.1528\end{array}$ & $\begin{array}{l}-8.427 \\
-8.389\end{array}$ & $\begin{array}{c}5.3045 \\
(1 ; 22) \\
5.3046 \\
(1 ; 22)\end{array}$ & [Si82b] \\
\hline${ }^{82} \mathrm{Se}$ & $\begin{array}{r}1946.854 \\
23 \\
1958.366 \\
19\end{array}$ & $\begin{array}{l}1946.829 \\
1958.383\end{array}$ & $\begin{array}{l}0.808 \\
0.814\end{array}$ & $\begin{array}{r}4.7179 \\
1\end{array}$ & 4.140 & $\begin{array}{l}0.0875 \\
0.0872\end{array}$ & $\begin{array}{l}2.1533 \\
2.1528\end{array}$ & $\begin{array}{l}-8.426 \\
-8.388\end{array}$ & $\begin{array}{c}5.3045 \\
(2 ; 21) \\
5.3047 \\
(2 ; 21)\end{array}$ & [Si82b] \\
\hline${ }^{70} \mathrm{Br}$ & $\begin{array}{r}2039.746 \\
20 \\
2052.798 \\
17\end{array}$ & $\begin{array}{l}2039.729 \\
2052.810\end{array}$ & $\begin{array}{l}0.728 \\
0.933\end{array}$ & $\begin{array}{r}4.7519 \\
1\end{array}$ & 4.163 & $\begin{array}{l}0.0893 \\
0.0890\end{array}$ & $\begin{array}{l}2.1570 \\
2.1564\end{array}$ & $\begin{array}{l}-7.792 \\
-7.756\end{array}$ & $\begin{array}{c}5.3337 \\
(2 ; 17) \\
5.3338 \\
(1 ; 22)\end{array}$ & [Ma83] \\
\hline${ }^{81} \mathrm{Br}$ & $\begin{array}{r}2040.214 \\
24 \\
2053.261 \\
20\end{array}$ & $\begin{array}{l}2040.209 \\
2053.265\end{array}$ & $\begin{array}{l}0.651 \\
0.827\end{array}$ & $\begin{array}{r}4.7474 \\
1\end{array}$ & 4.160 & $\begin{array}{l}0.0893 \\
0.0890\end{array}$ & $\begin{array}{l}2.1569 \\
2.1563\end{array}$ & $\begin{array}{l}-7.789 \\
-7.753\end{array}$ & $\begin{array}{c}5.3297 \\
(2 ; 15) \\
5.3299 \\
(2 ; 19)\end{array}$ & [Mas3] \\
\hline${ }^{78} \mathrm{Kr}$ & $\begin{array}{r}2130.917 \\
43 \\
2145.180 \\
40\end{array}$ & $\begin{array}{l}2130.896 \\
2145.198\end{array}$ & $\begin{array}{l}1.174 \\
1.183\end{array}$ & $\begin{array}{r}4.8099 \\
2\end{array}$ & 4.203 & $\begin{array}{l}0.0910 \\
0.0906\end{array}$ & $\begin{array}{l}2.1610 \\
2.1604\end{array}$ & $\begin{array}{l}-7.242 \\
-7.201\end{array}$ & $\begin{array}{c}5.3842 \\
(3 ; 26) \\
5.3844 \\
(3 ; 26)\end{array}$ & [Ma85] \\
\hline
\end{tabular}


TABLE IIIA. Muonic $2 p \rightarrow 1 s$ Transition Energies and Barrett Radii for $Z<60$ and $Z>77$ See page 194 for Explanation of Tables

\begin{tabular}{|c|c|c|c|c|c|c|c|c|c|c|}
\hline Isotope & $\begin{array}{l}E_{\text {exp. }} \\
{[\mathrm{keV}]}\end{array}$ & $\begin{array}{l}E_{\text {theo. }} \\
{[\mathrm{keV}]}\end{array}$ & $\begin{array}{l}\text { NPol } \\
{[\mathrm{keV}]}\end{array}$ & $\begin{array}{c}c \\
{[\mathrm{fm}]}\end{array}$ & $\underset{[\mathrm{fm}]}{\left\langle r^{2}\right\rangle_{\text {model }}^{1 / 2}}$ & $\begin{array}{c}\alpha \\
{[1 / f \mathrm{~m}]}\end{array}$ & k & $\begin{array}{c}C_{z} \\
{[\mathrm{am} / \mathrm{keV}]}\end{array}$ & $\begin{array}{l}R_{k a}^{\mu} \\
{[\mathrm{fm}]}\end{array}$ & Ref. \\
\hline${ }^{80} K r$ & $\begin{array}{r}2131.839 \\
44 \\
2146.091 \\
40\end{array}$ & $\begin{array}{l}2131.810 \\
2146.115\end{array}$ & $\begin{array}{l}1.064 \\
1.071\end{array}$ & $\begin{array}{r}4.8019 \\
3\end{array}$ & 4.198 & $\begin{array}{l}0.0910 \\
0.0907\end{array}$ & $\begin{array}{l}2.1609 \\
2.1603\end{array}$ & $\begin{array}{l}-7.235 \\
-7.199\end{array}$ & $\begin{array}{c}5.3771 \\
(3 ; 23) \\
5.3773 \\
(3 ; 23)\end{array}$ & [Ma85] \\
\hline${ }^{82} \mathrm{Kr}$ & $\begin{array}{r}2132.696 \\
43 \\
2146.976 \\
40\end{array}$ & $\begin{array}{l}3132.679 \\
2146.990\end{array}$ & $\begin{array}{l}0.931 \\
0.938\end{array}$ & $\begin{array}{r}4.7941 \\
2\end{array}$ & 4.192 & $\begin{array}{l}0.0911 \\
0.0907\end{array}$ & $\begin{array}{l}2.1607 \\
2.1601\end{array}$ & $\begin{array}{l}-7.235 \\
-7.195\end{array}$ & $\begin{array}{c}5.3701 \\
(3 ; 20) \\
5.3703 \\
(3 ; 20)\end{array}$ & {$[\mathrm{Ma} 85]$} \\
\hline${ }^{83} \mathrm{Kr}$ & $\begin{array}{r}2133.601 \\
52 \\
2148.108 \\
47\end{array}$ & $\begin{array}{l}2133.570 \\
2148.133\end{array}$ & $\begin{array}{l}0.683 \\
0.936\end{array}$ & $\begin{array}{r}4.7850 \\
3\end{array}$ & 4.186 & $\begin{array}{l}0.0911 \\
0.0908\end{array}$ & $\begin{array}{l}2.1606 \\
2.1600\end{array}$ & $\begin{array}{l}-7.228 \\
-7.193\end{array}$ & $\begin{array}{c}5.3621 \\
(4 ; 15) \\
5.3622 \\
(3 ; 20)\end{array}$ & [Ma85] \\
\hline${ }^{84} \mathrm{Kr}$ & $\begin{array}{r}2133.406 \\
41 \\
2147.574 \\
39\end{array}$ & $\begin{array}{l}2133.330 \\
2147.643\end{array}$ & $\begin{array}{l}0.833 \\
0.838\end{array}$ & $\begin{array}{r}4.7883 \\
2\end{array}$ & 4.188 & $\begin{array}{l}0.0911 \\
0.0908\end{array}$ & $\begin{array}{l}2.1607 \\
2.1601\end{array}$ & $\begin{array}{l}-7.230 \\
-7.194\end{array}$ & $\begin{array}{c}5.3651 \\
(3 ; 18) \\
5.3652 \\
(3 ; 18)\end{array}$ & [Ma85] \\
\hline${ }^{86} \mathrm{Kr}$ & $\begin{array}{r}2134.191 \\
35 \\
2148.498 \\
34\end{array}$ & $\begin{array}{l}2134.186 \\
2148.503\end{array}$ & $\begin{array}{l}0.861 \\
0.866\end{array}$ & $\begin{array}{r}4.7819 \\
2\end{array}$ & 4.184 & $\begin{array}{l}0.0911 \\
0.0908\end{array}$ & $\begin{array}{l}2.1606 \\
2.1600\end{array}$ & $\begin{array}{l}-7.225 \\
-7.189\end{array}$ & $\begin{array}{c}5.3594 \\
(3 ; 19) \\
5.3595 \\
(2 ; 19)\end{array}$ & [Ma85] \\
\hline${ }^{85} \mathrm{Rb}$ & $\begin{array}{r}2229.014 \\
12 \\
2244.964 \\
10\end{array}$ & $\begin{array}{l}2229.022 \\
2244.959\end{array}$ & $\begin{array}{l}0.770 \\
0.853\end{array}$ & $\begin{array}{r}4.8107 \\
1\end{array}$ & 4.204 & $\begin{array}{l}0.0929 \\
0.0926\end{array}$ & $\begin{array}{l}2.1641 \\
2.1634\end{array}$ & $\begin{array}{l}-6.722 \\
-6.688\end{array}$ & $\begin{array}{l}5.3840 \\
(1 ; 16) \\
5.3841 \\
(1 ; 17)\end{array}$ & $\begin{array}{l}{[\mathrm{He} 86]} \\
\text { [Ma83] }\end{array}$ \\
\hline${ }^{87} R b$ & $\begin{array}{r}2230.030 \\
20 \\
2245.904 \\
14\end{array}$ & $\begin{array}{l}2230.007 \\
2245.915\end{array}$ & $\begin{array}{l}0.758 \\
0.807\end{array}$ & $\begin{array}{r}4.8035 \\
1\end{array}$ & 4.199 & $\begin{array}{l}0.0930 \\
0.0926\end{array}$ & $\begin{array}{l}2.1640 \\
2.1633\end{array}$ & $\begin{array}{l}-6.721 \\
-6.682\end{array}$ & $\begin{array}{l}5.3775 \\
(1 ; 15) \\
5.3777 \\
(1 ; 16)\end{array}$ & $\begin{array}{l}{[\mathrm{He} 86]} \\
{[\mathrm{Ma} 83]}\end{array}$ \\
\hline${ }^{84} S r$ & $\begin{array}{r}2321.161 \\
33 \\
2338.677 \\
24\end{array}$ & $\begin{array}{l}2321.176 \\
2338.669\end{array}$ & $\begin{array}{l}1.125 \\
1.136\end{array}$ & $\begin{array}{r}4.8654 \\
2\end{array}$ & 4.241 & $\begin{array}{l}0.0946 \\
0.0942\end{array}$ & $\begin{array}{l}2.1680 \\
2.1673\end{array}$ & $\begin{array}{l}-6.285 \\
-6.247\end{array}$ & $\begin{array}{l}5.4317 \\
(2 ; 21) \\
5.4319 \\
(2 ; 21)\end{array}$ & $\begin{array}{l}{[\mathrm{He} 86]} \\
{[\mathrm{Ma} 83]}\end{array}$ \\
\hline
\end{tabular}


TABLE IIIA. Muonic $2 p \rightarrow 1 s$ Transition Energies and Barrett Radii for $Z<60$ and $Z>77$ See page 194 for Explanation of Tables

\begin{tabular}{|c|c|c|c|c|c|c|c|c|c|c|}
\hline Isotope & $\begin{array}{c}E_{\text {exp. }} \\
{[\mathrm{keV}]}\end{array}$ & $\begin{array}{l}E_{\text {theo }} \\
{[\mathrm{keV}]}\end{array}$ & $\begin{array}{l}\text { NPol } \\
\text { [keV] }\end{array}$ & $\begin{array}{c}\mathbf{c} \\
{[\mathrm{fm}]}\end{array}$ & $\begin{array}{c}\left\langle r^{2}\right\rangle_{\text {model }}^{1 / 2} \\
{[\mathrm{fm}]}\end{array}$ & $\begin{array}{c}\alpha \\
{[1 / \mathrm{fm}]}\end{array}$ & k & $\begin{array}{c}C_{3} \\
{[\mathrm{am} / \mathrm{keV}]}\end{array}$ & $\begin{array}{l}R_{k \alpha}^{\mu} \\
{[\mathrm{fm}]}\end{array}$ & Ref. \\
\hline${ }^{86} \mathrm{Sr}$ & $\begin{array}{r}2323.151 \\
14 \\
2340.660 \\
11\end{array}$ & $\begin{array}{l}2323.154 \\
2340.658\end{array}$ & $\begin{array}{l}0.920 \\
0.929\end{array}$ & $\begin{array}{r}4.8503 \\
1\end{array}$ & 4.231 & $\begin{array}{l}0.0946 \\
0.0943\end{array}$ & $\begin{array}{l}2.1678 \\
2.1671\end{array}$ & $\begin{array}{l}-6.274 \\
-6.240\end{array}$ & $\begin{array}{c}5.4183 \\
(1 ; 17) \\
5.4184 \\
(1 ; 17)\end{array}$ & $\begin{array}{l}\text { [He86] } \\
{[\mathrm{Ma83}]}\end{array}$ \\
\hline${ }^{87} \mathrm{Sr}$ & $\begin{array}{r}2324.412 \\
62 \\
2342.009 \\
49\end{array}$ & $\begin{array}{l}2324.396 \\
2342.019\end{array}$ & $\begin{array}{l}0.724 \\
0.843\end{array}$ & $\begin{array}{r}4.8403 \\
3\end{array}$ & 4.224 & $\begin{array}{l}0.0947 \\
0.0943\end{array}$ & $\begin{array}{l}2.1676 \\
2.1669\end{array}$ & $\begin{array}{l}-6.272 \\
-6.234\end{array}$ & $\begin{array}{l}5.4093 \\
(4 ; 14) \\
5.4095 \\
(3 ; 16)\end{array}$ & $\begin{array}{l}{[\mathrm{He} 86]} \\
{[\mathrm{Ma83}]}\end{array}$ \\
\hline${ }^{88} \mathrm{Sr}$ & $\begin{array}{r}2324.673 \\
10 \\
2342.192 \\
08\end{array}$ & $\begin{array}{l}2324.677 \\
2342.190\end{array}$ & $\begin{array}{l}0.929 \\
0.937\end{array}$ & $\begin{array}{r}4.8399 \\
1\end{array}$ & 4.224 & $\begin{array}{l}0.0947 \\
0.0943\end{array}$ & $\begin{array}{l}2.1676 \\
2.1669\end{array}$ & $\begin{array}{l}-6.272 \\
-6.233\end{array}$ & $\begin{array}{l}5.4090 \\
(1 ; 17) \\
5.4092 \\
(1 ; 18)\end{array}$ & $\begin{array}{l}{[\mathrm{He} 86]} \\
{[\mathrm{Ma83}]}\end{array}$ \\
\hline${ }^{80} Y$ & $\begin{array}{r}2420.793 \\
10 \\
2440.062 \\
9\end{array}$ & $\begin{array}{l}2420.789 \\
2440.065\end{array}$ & $\begin{array}{l}0.861 \\
0.867\end{array}$ & $\begin{array}{r}4.8672 \\
1\end{array}$ & 4.243 & $\begin{array}{l}0.0965 \\
0.0960\end{array}$ & $\begin{array}{l}2.1711 \\
2.1703\end{array}$ & $\begin{array}{l}-5.867 \\
-5.826\end{array}$ & $\begin{array}{l}5.4324 \\
(1 ; 15) \\
5.4326 \\
(1 ; 15)\end{array}$ & $\begin{array}{l}{[\mathrm{He} 86]} \\
{[\mathrm{Ma} 83]}\end{array}$ \\
\hline${ }^{80} \mathrm{Zr}$ & $\begin{array}{r}2515.368 \\
11 \\
2536.500 \\
10\end{array}$ & $\begin{array}{l}2515.362 \\
2536.505\end{array}$ & $\begin{array}{l}0.968 \\
0.975\end{array}$ & $\begin{array}{r}4.9075 \\
1\end{array}$ & 4.270 & $\begin{array}{l}0.0981 \\
0.0977\end{array}$ & $\begin{array}{l}2.1747 \\
2.1738\end{array}$ & $\begin{array}{l}-5.504 \\
-5.469\end{array}$ & $\begin{array}{l}5.4675 \\
(1 ; 16) \\
5.4676 \\
(1 ; 16)\end{array}$ & $\begin{array}{l}{[\mathrm{He} 86]} \\
{[\mathrm{Ma83}]}\end{array}$ \\
\hline${ }^{90} Z_{r} \dagger$ & $\begin{array}{r}2515.122 \\
23 \\
2536.237 \\
22\end{array}$ & $\begin{array}{l}2515.118 \\
2536.239\end{array}$ & $\begin{array}{l}1.083 \\
0.964\end{array}$ & $\begin{array}{r}4.9011 \\
2\end{array}$ & 4.272 & $\begin{array}{l}0.1029 \\
0.1029\end{array}$ & $\begin{array}{l}2.1950 \\
2.1970\end{array}$ & $\begin{array}{l}-5.510 \\
-5.470\end{array}$ & $\begin{array}{c}5.4684 \\
(1 ; 16) \\
5.4683 \\
(1 ; 16)\end{array}$ & [Ph85] \\
\hline${ }^{01} \mathrm{Zr}$ & $\begin{array}{r}2511.861 \\
43 \\
2533.047 \\
33\end{array}$ & $\begin{array}{l}2511.860 \\
2533.048\end{array}$ & $\begin{array}{l}0.880 \\
0.957\end{array}$ & $\begin{array}{r}4.9288 \\
2\end{array}$ & 4.285 & $\begin{array}{l}0.0980 \\
0.0975\end{array}$ & $\begin{array}{l}2.1751 \\
2.1742\end{array}$ & $\begin{array}{l}-5.513 \\
-5.474\end{array}$ & $\begin{array}{c}5.4864 \\
(2 ; 15) \\
5.4867 \\
(2 ; 16)\end{array}$ & $\begin{array}{l}{[\mathrm{He} 86]} \\
{[\mathrm{Ma} 83]}\end{array}$ \\
\hline${ }^{02} \mathrm{Zr}$ & $\begin{array}{r}2507.227 \\
18 \\
2528.268 \\
13\end{array}$ & $\begin{array}{l}2507.198 \\
2528.283\end{array}$ & $\begin{array}{l}0.976 \\
0.984\end{array}$ & $\begin{array}{r}4.9583 \\
1\end{array}$ & 4.305 & $\begin{array}{l}0.0978 \\
0.0974\end{array}$ & $\begin{array}{l}2.1755 \\
2.1747\end{array}$ & $\begin{array}{l}-5.525 \\
-5.488\end{array}$ & $\begin{array}{c}5.5129 \\
(1 ; 16) \\
5.5131 \\
(1 ; 16)\end{array}$ & $\begin{array}{l}{[\mathrm{He} 86]} \\
{[\mathrm{Ma} 83]}\end{array}$ \\
\hline
\end{tabular}

$\dagger$ Here, all data are taken from the quoted reference 
TABLE IIIA. Muonic $2 p \rightarrow 1 s$ Transition Energies and Barrett Radii for $Z<60$ and $Z>77$ See page 194 for Explanation of Tables

\begin{tabular}{|c|c|c|c|c|c|c|c|c|c|c|}
\hline Isotope & $\begin{array}{c}E_{\text {exp. }} \\
{[\mathrm{keV}]}\end{array}$ & $\begin{array}{l}E_{\text {theo }} \\
\text { [keV] }\end{array}$ & $\begin{array}{l}\text { NPol } \\
{[\mathrm{keV}]}\end{array}$ & $\underset{[\mathrm{fm}]}{\mathrm{c}}$ & $\begin{array}{c}\left\langle r^{2}\right\rangle_{\text {model }}^{1 / 2} \\
{[\mathrm{fm}]}\end{array}$ & $\begin{array}{c}\alpha \\
{[1 / \mathrm{fm}]}\end{array}$ & k & $\begin{array}{c}C_{z} \\
{[\mathrm{am} / \mathrm{keV}]}\end{array}$ & $\begin{array}{l}R_{k \alpha}^{\mu} \\
{[\mathrm{fm}]}\end{array}$ & Ref. \\
\hline${ }^{04} \mathrm{Zr}$ & $\begin{array}{r}2501.373 \\
21 \\
2522.386 \\
15\end{array}$ & $\begin{array}{l}2501.363 \\
2522.391\end{array}$ & $\begin{array}{l}0.953 \\
0.946\end{array}$ & $\begin{array}{r}4.9945 \\
1\end{array}$ & 4.330 & $\begin{array}{l}0.0976 \\
0.0972\end{array}$ & $\begin{array}{l}2.1761 \\
2.1753\end{array}$ & $\begin{array}{l}-5.541 \\
-5.503\end{array}$ & $\begin{array}{c}5.5453 \\
(1 ; 16) \\
5.5455 \\
(1 ; 16)\end{array}$ & $\begin{array}{l}\text { [He86] } \\
\text { [Ma83] }\end{array}$ \\
\hline${ }^{90} \mathrm{Zr}$ & $\begin{array}{r}2497.167 \\
49 \\
2518.081 \\
36\end{array}$ & $\begin{array}{l}2497.104 \\
2518.115\end{array}$ & $\begin{array}{l}0.959 \\
0.966\end{array}$ & $\begin{array}{r}5.0212 \\
2\end{array}$ & 4.349 & $\begin{array}{l}0.0975 \\
0.0970\end{array}$ & $\begin{array}{l}2.1766 \\
2.1757\end{array}$ & $\begin{array}{l}-5.553 \\
-5.512\end{array}$ & $\begin{array}{c}5.5692 \\
(3 ; 16) \\
5.5694 \\
(2 ; 16)\end{array}$ & $\begin{array}{l}{[\mathrm{He} 86]} \\
{[\mathrm{Ma83}]}\end{array}$ \\
\hline${ }^{93} \mathrm{Nb}$ & $\begin{array}{r}2603.418 \\
20 \\
2626.680 \\
16\end{array}$ & $\begin{array}{l}2603.461 \\
2626.653\end{array}$ & $\begin{array}{l}0.991 \\
1.060\end{array}$ & $\begin{array}{r}4.9853 \\
1\end{array}$ & 4.324 & $\begin{array}{l}0.0995 \\
0.0990\end{array}$ & $\begin{array}{l}2.1789 \\
2.1780\end{array}$ & $\begin{array}{l}-5.194 \\
-5.154\end{array}$ & $\begin{array}{c}5.5361 \\
(1 ; 15) \\
5.5363 \\
(1 ; 16)\end{array}$ & $\begin{array}{l}{[\mathrm{He} 86]} \\
{[\mathrm{Ma} 83]}\end{array}$ \\
\hline${ }^{02} \mathrm{Mo}$ & $\begin{array}{r}2706.804 \\
33 \\
2732.050 \\
23\end{array}$ & $\begin{array}{l}2706.806 \\
2732.049\end{array}$ & $\begin{array}{l}1.116 \\
1.127\end{array}$ & $\begin{array}{r}4.9754 \\
1\end{array}$ & 4.317 & $\begin{array}{l}0.1014 \\
0.1009\end{array}$ & $\begin{array}{l}2.1817 \\
2.1807\end{array}$ & $\begin{array}{l}-4.878 \\
-4.840\end{array}$ & $\begin{array}{c}5.5264 \\
(2 ; 16) \\
5.5266 \\
(1 ; 16)\end{array}$ & [Sc80b] \\
\hline${ }^{84} \mathrm{Mo}$ & $\begin{array}{r}2697.664 \\
23 \\
2722.739 \\
17\end{array}$ & $\begin{array}{l}2697.605 \\
2722.771\end{array}$ & $\begin{array}{l}1.191 \\
1.203\end{array}$ & $\begin{array}{r}5.0264 \\
1\end{array}$ & 4.353 & $\begin{array}{l}0.1011 \\
0.1006\end{array}$ & $\begin{array}{l}2.1825 \\
2.1815\end{array}$ & $\begin{array}{l}-4.899 \\
-4.861\end{array}$ & $\begin{array}{l}5.5720 \\
(1 ; 18) \\
5.5723 \\
(1 ; 18)\end{array}$ & {$[\mathrm{Sc} 80 \mathrm{~b}]$} \\
\hline${ }^{95} \mathrm{Mo}$ & $\begin{array}{r}2694.931 \\
17 \\
2720.072 \\
14\end{array}$ & $\begin{array}{l}2694.929 \\
2720.073\end{array}$ & $\begin{array}{l}1.105 \\
1.116\end{array}$ & $\begin{array}{r}5.0407 \\
1\end{array}$ & 4.362 & $\begin{array}{l}0.1011 \\
0.1005\end{array}$ & $\begin{array}{l}2.1828 \\
2.1818\end{array}$ & $\begin{array}{l}-4.908 \\
-4.865\end{array}$ & $\begin{array}{l}5.5848 \\
(1 ; 16) \\
5.5851 \\
(1 ; 16)\end{array}$ & [Sc80b] \\
\hline${ }^{96} \mathrm{Mo}$ & $\begin{array}{r}2689.546 \\
11 \\
2714.638 \\
11\end{array}$ & $\begin{array}{l}2689.542 \\
2714.642\end{array}$ & $\begin{array}{l}1.258 \\
1.272\end{array}$ & $\begin{array}{r}5.0711 \\
1\end{array}$ & 4.384 & $\begin{array}{l}0.1009 \\
0.1003\end{array}$ & $\begin{array}{l}2.1833 \\
2.1823\end{array}$ & $\begin{array}{l}-4.919 \\
-4.877\end{array}$ & $\begin{array}{l}5.6122 \\
(1 ; 19) \\
5.6125 \\
(1 ; 19)\end{array}$ & {$[\mathrm{Sc} 80 \mathrm{~b}]$} \\
\hline${ }^{97} \mathrm{Mo}$ & $\begin{array}{r}2688.468 \\
20 \\
2713.671 \\
16\end{array}$ & $\begin{array}{l}2688.456 \\
2713.679\end{array}$ & $\begin{array}{l}0.995 \\
1.139\end{array}$ & $\begin{array}{r}5.0757 \\
1\end{array}$ & 4.387 & $\begin{array}{l}0.1009 \\
0.1003\end{array}$ & $\begin{array}{l}2.1834 \\
2.1824\end{array}$ & $\begin{array}{l}-4.922 \\
-4.879\end{array}$ & $\begin{array}{c}5.6163 \\
(1 ; 15) \\
5.6166 \\
(1 ; 17)\end{array}$ & {$[\mathrm{Sc} 80 \mathrm{~b}]$} \\
\hline
\end{tabular}


TABLE IIIA. Muonic $2 p \rightarrow 1 s$ Transition Energies and Barrett Radii for $Z<60$ and $Z>77$ See page 194 for Explanation of Tables

\begin{tabular}{|c|c|c|c|c|c|c|c|c|c|c|}
\hline Isotope & $\begin{array}{l}E_{\text {exp. }} \\
{[\mathrm{keV}]}\end{array}$ & $\begin{array}{l}E_{\text {theo. }} \\
\text { [keV] }\end{array}$ & $\begin{array}{l}\text { NPol } \\
{[\mathrm{keV}]}\end{array}$ & $\begin{array}{c}c \\
{[\mathrm{fm}]}\end{array}$ & $\begin{array}{c}\left\langle r^{2}\right\rangle_{\text {model }}^{1 / 2} \\
{[\mathbf{f m}]}\end{array}$ & $\underset{[1 / f m]}{\alpha}$ & $\mathbf{k}$ & $\begin{array}{c}C_{z} \\
{[\mathrm{am} / \mathrm{keV}]}\end{array}$ & $\begin{array}{l}R_{k a}^{\mu} \\
{[\mathrm{fm}]}\end{array}$ & Ref. \\
\hline${ }^{98} \mathrm{Mo}$ & $\begin{array}{r}2683.347 \\
26 \\
2708.373 \\
19\end{array}$ & $\begin{array}{l}2683.335 \\
2708.379\end{array}$ & $\begin{array}{l}1.259 \\
1.272\end{array}$ & $\begin{array}{r}5.1054 \\
1\end{array}$ & 4.407 & $\begin{array}{l}0.1007 \\
0.1001\end{array}$ & $\begin{array}{l}2.1839 \\
2.1829\end{array}$ & $\begin{array}{l}-4.933 \\
-4.890\end{array}$ & $\begin{array}{l}5.6430 \\
(1 ; 19) \\
5.6433 \\
(1 ; 19)\end{array}$ & [Sc80b] \\
\hline${ }^{100} \mathrm{Mo}$ & $\begin{array}{r}2674.022 \\
26 \\
2698.955 \\
18\end{array}$ & $\begin{array}{l}2673.999 \\
2698.966\end{array}$ & $\begin{array}{l}1.398 \\
1.411\end{array}$ & $\begin{array}{r}5.1564 \\
1\end{array}$ & 4.444 & $\begin{array}{l}0.1004 \\
0.0998\end{array}$ & $\begin{array}{l}2.1848 \\
2.1838\end{array}$ & $\begin{array}{l}-4.954 \\
-4.911\end{array}$ & $\begin{array}{l}5.6901 \\
(1 ; 21) \\
5.6904 \\
(1 ; 21)\end{array}$ & [Sc80b] \\
\hline${ }^{88} R u$ & $\begin{array}{r}2889.893 \\
228 \\
2919.669 \\
29\end{array}$ & $\begin{array}{l}2889.925 \\
2919.668\end{array}$ & $\begin{array}{l}1.316 \\
1.332\end{array}$ & $\begin{array}{r}5.0845 \\
1\end{array}$ & 4.393 & $\begin{array}{l}0.1044 \\
0.1038\end{array}$ & $\begin{array}{l}2.1893 \\
2.1881\end{array}$ & $\begin{array}{l}-4.379 \\
-4.339\end{array}$ & $\begin{array}{l}5.6224 \\
(10 ; 17) \\
5.6226 \\
(1 ; 17)\end{array}$ & [Ha89] \\
\hline${ }^{28} R u$ & $\begin{array}{r}2880.842 \\
530 \\
2910.801 \\
230\end{array}$ & $\begin{array}{l}2881.096 \\
2910.754\end{array}$ & $\begin{array}{l}1.389 \\
1.405\end{array}$ & $\begin{array}{r}5.1281 \\
10\end{array}$ & 4.423 & $\begin{array}{l}0.1042 \\
0.1035\end{array}$ & $\begin{array}{l}2.1900 \\
2.1889\end{array}$ & $\begin{array}{l}-4.399 \\
-4.354\end{array}$ & $\begin{array}{l}5.6616 \\
(23 ; 18) \\
5.6620 \\
(10 ; 18)\end{array}$ & [Ha89] \\
\hline${ }^{\theta \theta} R u$ & $\begin{array}{r}2877.569 \\
78 \\
2907.233 \\
50\end{array}$ & $\begin{array}{l}2877.585 \\
2907.227\end{array}$ & $\begin{array}{l}1.271 \\
1.238\end{array}$ & $\begin{array}{r}5.1448 \\
2\end{array}$ & 4.435 & $\begin{array}{l}0.1041 \\
0.1034\end{array}$ & $\begin{array}{l}2.1903 \\
2.1892\end{array}$ & $\begin{array}{l}-4.405 \\
-4.360\end{array}$ & $\begin{array}{l}5.6767 \\
(3 ; 17) \\
5.6770 \\
(2 ; 16)\end{array}$ & [Ha89] \\
\hline${ }^{100} R u$ & $\begin{array}{r}2872.289 \\
79 \\
2901.977 \\
62\end{array}$ & $\begin{array}{l}2872.362 \\
2901.932\end{array}$ & $\begin{array}{l}1.458 \\
1.472\end{array}$ & $\begin{array}{r}5.1714 \\
2\end{array}$ & 4.453 & $\begin{array}{l}0.1039 \\
0.1033\end{array}$ & $\begin{array}{l}2.1908 \\
2.1896\end{array}$ & $\begin{array}{l}-4.414 \\
-4.373\end{array}$ & $\begin{array}{l}5.7007 \\
(4 ; 19) \\
5.7010 \\
(3 ; 19)\end{array}$ & [Ha89] \\
\hline${ }^{101} R u$ & $\begin{array}{r}2869.600 \\
63 \\
2899.563 \\
102\end{array}$ & $\begin{array}{l}2869.606 \\
2899.548\end{array}$ & $\begin{array}{l}1.034 \\
1.443\end{array}$ & $\begin{array}{r}5.1829 \\
3\end{array}$ & 4.461 & $\begin{array}{l}0.1038 \\
0.1032\end{array}$ & $\begin{array}{l}2.1910 \\
2.1898\end{array}$ & $\begin{array}{l}-4.418 \\
-4.377\end{array}$ & $\begin{array}{l}5.7111 \\
(3 ; 14) \\
5.7114 \\
(5 ; 19)\end{array}$ & [Ha89] \\
\hline${ }^{102} R u$ & $\begin{array}{r}2864.404 \\
35 \\
2893.906 \\
29\end{array}$ & $\begin{array}{l}2864.412 \\
2893.900\end{array}$ & $\begin{array}{l}1.547 \\
1.557\end{array}$ & $\begin{array}{r}5.2110 \\
1\end{array}$ & 4.481 & $\begin{array}{l}0.1037 \\
0.1030\end{array}$ & $\begin{array}{l}2.1915 \\
2.1903\end{array}$ & $\begin{array}{l}-4.431 \\
-4.386\end{array}$ & $\begin{array}{l}5.7365 \\
(2 ; 21) \\
5.7368 \\
(1 ; 20)\end{array}$ & [Ha89] \\
\hline
\end{tabular}


TABLE IIIA. Muonic $2 p \rightarrow 1 s$ Transition Energies and Barrett Radii for $Z<60$ and $Z>77$ See page 194 for Explanation of Tables

\begin{tabular}{|c|c|c|c|c|c|c|c|c|c|c|}
\hline Isotope & $\begin{array}{c}E_{\text {exp. }} \\
{[\mathrm{keV}]}\end{array}$ & $\begin{array}{l}E_{\text {theo. }} \\
{[\mathrm{keV}]}\end{array}$ & $\begin{array}{l}\text { NPol } \\
{[\mathrm{keV}]}\end{array}$ & $\begin{array}{c}\mathrm{c} \\
{[\mathrm{fm}]}\end{array}$ & $\begin{array}{c}\left\langle r^{2}\right\rangle_{\text {model }}^{1 / 2} \\
{[\mathrm{fm}]}\end{array}$ & $\begin{array}{c}\alpha \\
{[1 / f m]}\end{array}$ & $\mathbf{k}$ & $\begin{array}{c}C_{z} \\
{[\mathrm{am} / \mathrm{keV}]}\end{array}$ & $\begin{array}{l}R_{k \alpha}^{\mu} \\
{[\mathrm{fm}]}\end{array}$ & Ref. \\
\hline${ }^{104} R u$ & $\begin{array}{r}2856.267 \\
45 \\
2885.657 \\
37\end{array}$ & $\begin{array}{l}2856.265 \\
2885.659\end{array}$ & $\begin{array}{l}1.618 \\
1.614\end{array}$ & $\begin{array}{r}5.2516 \\
2\end{array}$ & 4.509 & $\begin{array}{l}0.1034 \\
0.1028\end{array}$ & $\begin{array}{l}2.1922 \\
2.1910\end{array}$ & $\begin{array}{l}-4.445 \\
-4.404\end{array}$ & $\begin{array}{l}5.7732 \\
(2 ; 22) \\
5.7735 \\
(2 ; 22)\end{array}$ & [Ha89] \\
\hline${ }^{109} R h$ & $\begin{array}{r}2961.294 \\
30 \\
2993.146 \\
22\end{array}$ & $\begin{array}{l}2961.240 \\
2993.175\end{array}$ & $\begin{array}{l}1.439 \\
1.431\end{array}$ & $\begin{array}{r}5.2293 \\
1\end{array}$ & 4.494 & $\begin{array}{l}0.1053 \\
0.1046\end{array}$ & $\begin{array}{l}2.1946 \\
2.1934\end{array}$ & $\begin{array}{l}-4.198 \\
-4.153\end{array}$ & $\begin{array}{l}5.7521 \\
(1 ; 18) \\
5.7525 \\
(1 ; 18)\end{array}$ & [Ha89] \\
\hline${ }^{102} P d$ & $\begin{array}{r}3065.579 \\
109 \\
3100.193 \\
80\end{array}$ & $\begin{array}{l}3065.568 \\
3100.199\end{array}$ & $\begin{array}{l}1.517 \\
1.532\end{array}$ & $\begin{array}{r}5.2156 \\
3\end{array}$ & 4.484 & $\begin{array}{l}0.1072 \\
0.1064\end{array}$ & $\begin{array}{l}2.1972 \\
2.1959\end{array}$ & $\begin{array}{l}-3.974 \\
-3.928\end{array}$ & $\begin{array}{l}5.7388 \\
(4 ; 19) \\
5.7392 \\
(3 ; 19)\end{array}$ & [Ha89] \\
\hline${ }^{104} P d$ & $\begin{array}{r}3057.604 \\
139 \\
3092.152 \\
120\end{array}$ & $\begin{array}{l}3057.606 \\
3092.150\end{array}$ & $\begin{array}{l}1.537 \\
1.553\end{array}$ & $\begin{array}{r}5.2509 \\
4\end{array}$ & 4.509 & $\begin{array}{l}0.1069 \\
0.1062\end{array}$ & $\begin{array}{l}2.1978 \\
2.1965\end{array}$ & $\begin{array}{l}-3.985 \\
-3.942\end{array}$ & $\begin{array}{l}5.7708 \\
(6 ; 18) \\
5.7712 \\
(5 ; 18)\end{array}$ & [Ha89] \\
\hline${ }^{105} P d$ & $\begin{array}{r}3054.538 \\
104 \\
3090.021 \\
149\end{array}$ & $\begin{array}{l}3054.551 \\
3089.995\end{array}$ & $\begin{array}{l}0.821 \\
1.764\end{array}$ & $\begin{array}{r}5.2613 \\
4\end{array}$ & 4.516 & $\begin{array}{l}0.1069 \\
0.1062\end{array}$ & $\begin{array}{l}2.1980 \\
2.1967\end{array}$ & $\begin{array}{l}-3.991 \\
-3.948\end{array}$ & $\begin{array}{l}5.7802 \\
(4 ; 10) \\
5.7806 \\
(6 ; 21)\end{array}$ & [Ha89] \\
\hline${ }^{108} P d$ & $\begin{array}{r}3050.087 \\
76 \\
3084.545 \\
53\end{array}$ & $\begin{array}{l}3050.088 \\
3084.544\end{array}$ & $\begin{array}{l}1.619 \\
1.632\end{array}$ & $\begin{array}{r}5.2847 \\
2\end{array}$ & 4.532 & $\begin{array}{l}0.1067 \\
0.1060\end{array}$ & $\begin{array}{l}2.1984 \\
2.1971\end{array}$ & $\begin{array}{l}-3.999 \\
-3.955\end{array}$ & $\begin{array}{l}5.8014 \\
(3 ; 19) \\
5.8017 \\
(2 ; 19)\end{array}$ & [Ha89] \\
\hline${ }^{108} P d$ & $\begin{array}{r}3042.551 \\
72 \\
3076.847 \\
47\end{array}$ & $\begin{array}{l}3042.504 \\
3076.867\end{array}$ & $\begin{array}{l}1.632 \\
1.637\end{array}$ & $\begin{array}{r}5.3184 \\
2\end{array}$ & 4.556 & $\begin{array}{l}0.1065 \\
0.1058\end{array}$ & $\begin{array}{l}2.1990 \\
2.1976\end{array}$ & $\begin{array}{l}-4.012 \\
-3.969\end{array}$ & $\begin{array}{l}5.8320 \\
(3 ; 20) \\
5.8324 \\
(2 ; 20)\end{array}$ & [Ha89] \\
\hline${ }^{110} P d$ & $\begin{array}{r}3035.646 \\
138 \\
3069.941 \\
88\end{array}$ & $\begin{array}{l}3035.663 \\
3069.934\end{array}$ & $\begin{array}{l}1.654 \\
1.643\end{array}$ & $\begin{array}{r}5.3490 \\
3\end{array}$ & 4.577 & $\begin{array}{l}0.1064 \\
0.1056\end{array}$ & $\begin{array}{l}2.1995 \\
2.1982\end{array}$ & $\begin{array}{l}-4.027 \\
-3.980\end{array}$ & $\begin{array}{l}5.8597 \\
(6 ; 20) \\
5.8601 \\
(4 ; 20)\end{array}$ & [Ha89] \\
\hline
\end{tabular}


TABLE IIIA. Muonic $2 p \rightarrow 1 s$ Transition Energies and Barrett Radii for $Z<60$ and $Z>77$ See page 194 for Explanation of Tables

\begin{tabular}{|c|c|c|c|c|c|c|c|c|c|c|}
\hline Isotope & $\begin{array}{l}E_{\text {exp. }} \\
{[\mathrm{keV}]}\end{array}$ & $\begin{array}{l}E_{\text {theo. }} \\
{[\mathrm{keV}]}\end{array}$ & $\begin{array}{l}\text { NPol } \\
{[\mathrm{keV}]}\end{array}$ & $\begin{array}{c}c \\
{[\mathrm{fm}]}\end{array}$ & $\begin{array}{c}\left\langle r^{2}\right\rangle_{\text {model }}^{1 / 2} \\
{[\mathrm{fm}]}\end{array}$ & $\begin{array}{c}\alpha \\
{[1 / \mathrm{fm}]}\end{array}$ & k & $\begin{array}{c}C_{z} \\
{[\mathrm{am} / \mathrm{keV}]}\end{array}$ & $\begin{array}{l}R_{k a}^{\mu} \\
{[\mathrm{fm}]}\end{array}$ & Ref. \\
\hline${ }^{107} \mathrm{Ag}$ & $\begin{array}{r}3147.135 \\
28 \\
3184.302 \\
21\end{array}$ & $\begin{array}{l}3147.140 \\
3184.299\end{array}$ & $\begin{array}{l}1.487 \\
1.485\end{array}$ & $\begin{array}{r}5.3006 \\
1\end{array}$ & 4.544 & $\begin{array}{l}0.1084 \\
0.1076\end{array}$ & $\begin{array}{l}2.2015 \\
2.2000\end{array}$ & $\begin{array}{l}-3.804 \\
-3.759\end{array}$ & $\begin{array}{c}5.8149 \\
(1 ; 17) \\
5.8153 \\
(1 ; 17)\end{array}$ & [Ha89] \\
\hline${ }^{100} \mathrm{Ag}$ & $\begin{array}{r}3140.061 \\
31 \\
3177.124 \\
23\end{array}$ & $\begin{array}{l}3140.048 \\
3177.131\end{array}$ & $\begin{array}{l}1.483 \\
1.478\end{array}$ & $\begin{array}{r}5.3306 \\
1\end{array}$ & 4.565 & $\begin{array}{l}0.1082 \\
0.1074\end{array}$ & $\begin{array}{l}2.2020 \\
2.2006\end{array}$ & $\begin{array}{l}-3.815 \\
-3.769\end{array}$ & $\begin{array}{l}5.8421 \\
(1 ; 17) \\
5.8425 \\
(1 ; 17)\end{array}$ & [Ha89] \\
\hline${ }^{106} \mathrm{Cd}$ & $\begin{array}{r}3251.740 \\
109 \\
3291.755 \\
66\end{array}$ & $\begin{array}{l}3251.659 \\
3291.784\end{array}$ & $\begin{array}{l}1.546 \\
1.567\end{array}$ & $\begin{array}{r}5.2875 \\
2\end{array}$ & 4.534 & $\begin{array}{l}0.1102 \\
0.1094\end{array}$ & $\begin{array}{l}2.2040 \\
2.2025\end{array}$ & $\begin{array}{l}-3.611 \\
-3.567\end{array}$ & $\begin{array}{l}5.8022 \\
(4 ; 17) \\
5.8026 \\
(2 ; 17)\end{array}$ & [Fr87] \\
\hline${ }^{108} \mathrm{Cd}$ & $\begin{array}{r}3244.736 \\
74 \\
3284.706 \\
61\end{array}$ & $\begin{array}{l}3244.699 \\
3284.731\end{array}$ & $\begin{array}{l}1.529 \\
1.547\end{array}$ & $\begin{array}{r}5.3154 \\
2\end{array}$ & 4.554 & $\begin{array}{l}0.1101 \\
0.1093\end{array}$ & $\begin{array}{l}2.2046 \\
2.2031\end{array}$ & $\begin{array}{l}-3.622 \\
-3.577\end{array}$ & $\begin{array}{l}5.8275 \\
(3 ; 17) \\
5.8279 \\
(2 ; 17)\end{array}$ & [Fr87] \\
\hline${ }^{110} C d$ & $\begin{array}{r}3237.749 \\
47 \\
3277.678 \\
40\end{array}$ & $\begin{array}{l}3237.741 \\
3277.684\end{array}$ & $\begin{array}{l}1.556 \\
1.573\end{array}$ & $\begin{array}{r}5.3435 \\
1\end{array}$ & 4.574 & $\begin{array}{l}0.1099 \\
0.1091\end{array}$ & $\begin{array}{l}2.2051 \\
2.2036\end{array}$ & $\begin{array}{l}-3.632 \\
-3.587\end{array}$ & $\begin{array}{l}5.8530 \\
(2 ; 17) \\
5.8534 \\
(1 ; 17)\end{array}$ & [Fr87] \\
\hline${ }^{111} C d$ & $\begin{array}{r}3235.848 \\
59 \\
3275.805 \\
43\end{array}$ & $\begin{array}{l}3235.873 \\
3275.792\end{array}$ & $\begin{array}{l}1.434 \\
1.444\end{array}$ & $\begin{array}{r}5.3506 \\
1\end{array}$ & 4.579 & $\begin{array}{l}0.1098 \\
0.1089\end{array}$ & $\begin{array}{l}2.2051 \\
2.2035\end{array}$ & $\begin{array}{l}-3.636 \\
-3.589\end{array}$ & $\begin{array}{l}5.8594 \\
(2 ; 16) \\
5.8598 \\
(2 ; 16)\end{array}$ & [Fr87] \\
\hline${ }^{112} \mathrm{Cd}$ & $\begin{array}{r}3230.988 \\
39 \\
3270.895 \\
30\end{array}$ & $\begin{array}{l}3231.015 \\
3270.879\end{array}$ & $\begin{array}{l}1.578 \\
1.596\end{array}$ & $\begin{array}{r}5.3707 \\
1\end{array}$ & 4.593 & $\begin{array}{l}0.1096 \\
0.1088\end{array}$ & $\begin{array}{l}2.2054 \\
2.2039\end{array}$ & $\begin{array}{l}-3.643 \\
-3.598\end{array}$ & $\begin{array}{l}5.8777 \\
(1 ; 17) \\
5.8781 \\
(1 ; 17)\end{array}$ & [Fr87] \\
\hline${ }^{113} \mathrm{Cd}$ & $\begin{array}{r}3229.189 \\
53 \\
3268.959 \\
40\end{array}$ & $\begin{array}{l}3229.151 \\
3268.980\end{array}$ & $\begin{array}{l}1.393 \\
1.400\end{array}$ & $\begin{array}{r}5.3775 \\
1\end{array}$ & 4.597 & $\begin{array}{l}0.1096 \\
0.1088\end{array}$ & $\begin{array}{l}2.2056 \\
2.2041\end{array}$ & $\begin{array}{l}-3.644 \\
-3.599\end{array}$ & $\begin{array}{c}5.8839 \\
(2 ; 15) \\
5.8843 \\
(1 ; 15)\end{array}$ & [Fr87] \\
\hline
\end{tabular}




\section{G. FRICKE et al. Nuclear Charge Radii}

TABLE IIIA. Muonic $2 p \rightarrow 1 s$ Transition Energies and Barrett Radii for $Z<60$ and $Z>77$ See page 194 for Explanation of Tables

\begin{tabular}{|c|c|c|c|c|c|c|c|c|c|c|}
\hline Isotope & $\begin{array}{c}E_{\text {exp. }} \\
{[\mathrm{keV}]}\end{array}$ & $\begin{array}{l}E_{\text {theo. }} \\
{[\mathrm{keV}]}\end{array}$ & $\begin{array}{l}\text { NPol } \\
{[\mathrm{keV}]}\end{array}$ & $\begin{array}{c}\mathrm{c} \\
{[\mathrm{fm}]}\end{array}$ & $\begin{array}{c}\left\langle r^{2}\right\rangle_{\text {model }}^{1 / 2} \\
{[\mathrm{fm}]}\end{array}$ & $\begin{array}{c}\alpha \\
{[1 / f \mathbf{f m}]}\end{array}$ & $\mathbf{k}$ & $\begin{array}{c}C_{k} \\
{[\mathrm{am} / \mathrm{keV}]}\end{array}$ & $\begin{array}{l}R_{k a}^{\mu} \\
{[\mathrm{fm}]}\end{array}$ & Ref. \\
\hline${ }^{114} \mathrm{Cd}$ & $\begin{array}{r}3224.852 \\
31 \\
3264.699 \\
25\end{array}$ & $\begin{array}{l}3224.887 \\
3264.677\end{array}$ & $\begin{array}{l}1.576 \\
1.593\end{array}$ & $\begin{array}{r}5.3954 \\
1\end{array}$ & 4.610 & $\begin{array}{l}0.1093 \\
0.1085\end{array}$ & $\begin{array}{l}2.2056 \\
2.2041\end{array}$ & $\begin{array}{l}-3.653 \\
-3.607\end{array}$ & $\begin{array}{c}5.9002 \\
(1 ; 17) \\
5.9006 \\
(1 ; 17)\end{array}$ & [Fr87] \\
\hline${ }^{116} \mathrm{Cd}$ & $\begin{array}{r}3219.689 \\
37 \\
3259.454 \\
31\end{array}$ & $\begin{array}{l}3219.718 \\
3259.434\end{array}$ & $\begin{array}{l}1.593 \\
1.606\end{array}$ & $\begin{array}{r}5.4164 \\
1\end{array}$ & 4.625 & $\begin{array}{l}0.1093 \\
0.1085\end{array}$ & $\begin{array}{l}2.2061 \\
2.2046\end{array}$ & $\begin{array}{l}-3.661 \\
-3.616\end{array}$ & $\begin{array}{l}5.9193 \\
(1 ; 17) \\
5.9197 \\
(1 ; 17)\end{array}$ & [Fr87] \\
\hline${ }^{113} I n$ & $\begin{array}{r}3329.129 \\
397 \\
3372.659 \\
338\end{array}$ & $\begin{array}{l}3328.947 \\
3372.789\end{array}$ & $\begin{array}{l}0.928 \\
1.935\end{array}$ & $\begin{array}{r}5.3789 \\
10\end{array}$ & 4.598 & $\begin{array}{l}0.1113 \\
0.1105\end{array}$ & $\begin{array}{l}2.2084 \\
2.2067\end{array}$ & $\begin{array}{l}-3.471 \\
-3.428\end{array}$ & $\begin{array}{c}5.8843 \\
(14 ; 10) \\
5.8846 \\
(12 ; 20)\end{array}$ & [Ja89] \\
\hline${ }^{115} \mathrm{In}$ & $\begin{array}{r}3322.991 \\
32 \\
3366.759 \\
21\end{array}$ & $\begin{array}{l}3322.987 \\
3366.761\end{array}$ & $\begin{array}{l}0.915 \\
1.933\end{array}$ & $\begin{array}{r}5.4018 \\
1\end{array}$ & 4.614 & $\begin{array}{l}0.1112 \\
0.1103\end{array}$ & $\begin{array}{l}2.2088 \\
2.2072\end{array}$ & $\begin{array}{l}-3.481 \\
-3.434\end{array}$ & $\begin{array}{l}5.9051 \\
(1 ; 10) \\
5.9055 \\
(1 ; 20)\end{array}$ & [Ja89] \\
\hline${ }^{112} S n$ & $\begin{array}{r}3432.564 \\
60 \\
3478.531 \\
56\end{array}$ & $\begin{array}{l}3432.516 \\
3478.572\end{array}$ & $\begin{array}{l}1.537 \\
1.565\end{array}$ & $\begin{array}{r}5.3714 \\
2\end{array}$ & 4.593 & $\begin{array}{l}0.1131 \\
0.1121\end{array}$ & $\begin{array}{l}2.2109 \\
2.2092\end{array}$ & $\begin{array}{l}-3.310 \\
-3.261\end{array}$ & $\begin{array}{l}5.8765 \\
(2 ; 15) \\
5.8770 \\
(2 ; 15)\end{array}$ & [Pi90] \\
\hline${ }^{114} S n$ & $\begin{array}{r}3426.613 \\
71 \\
3471.921 \\
68\end{array}$ & $\begin{array}{l}3426.266 \\
3472.235\end{array}$ & $\begin{array}{l}1.539 \\
1.568\end{array}$ & $\begin{array}{r}5.3943 \\
2\end{array}$ & 4.609 & $\begin{array}{l}0.1129 \\
0.1120\end{array}$ & $\begin{array}{l}2.2113 \\
2.2096\end{array}$ & $\begin{array}{l}-3.317 \\
-3.271\end{array}$ & $\begin{array}{l}5.8974 \\
(2 ; 15) \\
5.8979 \\
(2 ; 15)\end{array}$ & {$[\mathrm{Pi} 90]$} \\
\hline${ }^{110} S n$ & $\begin{array}{r}3420.091 \\
62 \\
3465.757 \\
58\end{array}$ & $\begin{array}{l}3419.977 \\
3465.856\end{array}$ & $\begin{array}{l}1.510 \\
1.539\end{array}$ & $\begin{array}{r}5.4173 \\
2\end{array}$ & 4.625 & $\begin{array}{l}0.1128 \\
0.1119\end{array}$ & $\begin{array}{l}2.2118 \\
2.2101\end{array}$ & $\begin{array}{l}-3.326 \\
-3.280\end{array}$ & $\begin{array}{c}5.9183 \\
(2 ; 15) \\
5.9188 \\
(2 ; 15)\end{array}$ & (Pi90) \\
\hline${ }^{117} S n$ & $\begin{array}{r}3418.140 \\
63 \\
3463.810 \\
60\end{array}$ & $\begin{array}{l}3418.024 \\
3463.914\end{array}$ & $\begin{array}{l}1.407 \\
1.473\end{array}$ & $\begin{array}{r}5.4241 \\
2\end{array}$ & 4.630 & $\begin{array}{l}0.1127 \\
0.1118\end{array}$ & $\begin{array}{l}2.2119 \\
2.2102\end{array}$ & $\begin{array}{l}-3.327 \\
-3.281\end{array}$ & $\begin{array}{c}5.9246 \\
(2 ; 14) \\
5.9250 \\
(2 ; 14)\end{array}$ & [Pi90] \\
\hline
\end{tabular}


TABLE IIIA. Muonic $2 p \rightarrow 1 s$ Transition Energies and Barrett Radii for $Z<60$ and $Z>77$ See page 194 for Explanation of Tables

\begin{tabular}{|c|c|c|c|c|c|c|c|c|c|c|}
\hline Isotope & $\begin{array}{l}E_{\text {exp. }} \\
{[\mathrm{keV}]}\end{array}$ & $\begin{array}{l}E_{\text {theo. }} \\
{[\mathrm{keV}]}\end{array}$ & $\begin{array}{l}\text { NPol } \\
{[\mathrm{keV}]}\end{array}$ & $\begin{array}{c}\mathrm{c} \\
{[\mathrm{fm}]}\end{array}$ & $\begin{array}{c}\left\langle r^{2}\right\rangle_{\text {model }}^{1 / 2} \\
{[\mathrm{fm}]}\end{array}$ & $\begin{array}{c}\alpha \\
{[1 / \mathrm{fm}]}\end{array}$ & $\mathbf{k}$ & $\begin{array}{c}C_{z} \\
{[\mathrm{am} / \mathrm{keV}]}\end{array}$ & $\begin{array}{l}R_{k \alpha}^{\mu} \\
{[\mathrm{fm}]}\end{array}$ & Ref. \\
\hline${ }^{118} S n$ & $\begin{array}{r}3414.352 \\
112 \\
3459.748 \\
95\end{array}$ & $\begin{array}{l}3414.118 \\
3459.914\end{array}$ & $\begin{array}{l}1.554 \\
1.584\end{array}$ & $\begin{array}{r}5.4391 \\
3\end{array}$ & 4.641 & $\begin{array}{l}0.1126 \\
0.1117\end{array}$ & $\begin{array}{l}2.2121 \\
2.2104\end{array}$ & $\begin{array}{l}-3.333 \\
-3.286\end{array}$ & $\begin{array}{l}5.9381 \\
(4 ; 15) \\
5.9386 \\
(3 ; 15)\end{array}$ & [Be93] \\
\hline${ }^{110} S n$ & $\begin{array}{r}3412.848 \\
61 \\
3458.780 \\
60\end{array}$ & $\begin{array}{l}3412.928 \\
3458.703\end{array}$ & $\begin{array}{l}1.452 \\
1.477\end{array}$ & $\begin{array}{r}5.4431 \\
2\end{array}$ & 4.643 & $\begin{array}{l}0.1126 \\
0.1117\end{array}$ & $\begin{array}{l}2.2122 \\
2.2105\end{array}$ & $\begin{array}{l}-3.335 \\
-3.288\end{array}$ & $\begin{array}{l}5.9418 \\
(2 ; 14) \\
5.9423 \\
(2 ; 14)\end{array}$ & [Pi90] \\
\hline${ }^{120} S n$ & $\begin{array}{r}3408.975 \\
52 \\
3454.453 \\
50\end{array}$ & $\begin{array}{l}3408.849 \\
3454.568\end{array}$ & $\begin{array}{l}1.637 \\
1.667\end{array}$ & $\begin{array}{r}5.4588 \\
1\end{array}$ & 4.655 & $\begin{array}{l}0.1125 \\
0.1116\end{array}$ & $\begin{array}{l}2.2125 \\
2.2108\end{array}$ & $\begin{array}{l}-3.340 \\
-3.294\end{array}$ & $\begin{array}{l}5.9561 \\
(2 ; 16) \\
5.9566 \\
(2 ; 16)\end{array}$ & [Pi90] \\
\hline${ }^{122} S n$ & $\begin{array}{r}3404.231 \\
124 \\
3449.638 \\
107\end{array}$ & $\begin{array}{l}3404.091 \\
3449.741\end{array}$ & $\begin{array}{l}1.548 \\
1.576\end{array}$ & $\begin{array}{r}5.4761 \\
3\end{array}$ & 4.667 & $\begin{array}{l}0.1124 \\
0.1115\end{array}$ & $\begin{array}{l}2.2128 \\
2.2111\end{array}$ & $\begin{array}{l}-3.347 \\
-3.300\end{array}$ & $\begin{array}{l}5.9718 \\
(4 ; 15) \\
5.9723 \\
(4 ; 15)\end{array}$ & [Be93] \\
\hline${ }^{124} S n$ & $\begin{array}{r}3400.182 \\
61 \\
3445.482 \\
57\end{array}$ & $\begin{array}{l}3400.025 \\
3445.617\end{array}$ & $\begin{array}{l}1.454 \\
1.481\end{array}$ & $\begin{array}{r}5.4907 \\
2\end{array}$ & 4.677 & $\begin{array}{l}0.1123 \\
0.1114\end{array}$ & $\begin{array}{l}2.2131 \\
2.2114\end{array}$ & $\begin{array}{l}-3.352 \\
-3.305\end{array}$ & $\begin{array}{l}5.9853 \\
(2 ; 15) \\
5.9857 \\
(2 ; 15)\end{array}$ & {$[\mathrm{Pi} 90]$} \\
\hline${ }^{121} S b$ & $\begin{array}{r}3497.991 \\
58 \\
3547.434 \\
56\end{array}$ & $\begin{array}{l}3498.091 \\
3547.342\end{array}$ & $\begin{array}{l}1.354 \\
1.800\end{array}$ & $\begin{array}{r}5.4963 \\
2\end{array}$ & 4.681 & $\begin{array}{l}0.1139 \\
0.1130\end{array}$ & $\begin{array}{l}2.2159 \\
2.2141\end{array}$ & $\begin{array}{l}-3.203 \\
-3.157\end{array}$ & $\begin{array}{l}5.9895 \\
(2 ; 13) \\
5.9899 \\
(2 ; 17)\end{array}$ & [K188] \\
\hline${ }^{123} \mathrm{Sb}$ & $\begin{array}{r}3494.562 \\
78 \\
3544.247 \\
56\end{array}$ & $\begin{array}{l}3494.553 \\
3544.252\end{array}$ & $\begin{array}{l}1.063 \\
2.006\end{array}$ & $\begin{array}{r}5.5078 \\
2\end{array}$ & 4.689 & $\begin{array}{l}0.1139 \\
0.1129\end{array}$ & $\begin{array}{l}2.2161 \\
2.2143\end{array}$ & $\begin{array}{l}-3.209 \\
-3.161\end{array}$ & $\begin{array}{l}5.9999 \\
(3 ; 10) \\
6.0004 \\
(2 ; 19)\end{array}$ & [K188] \\
\hline${ }^{122} \mathrm{Te}$ & $\begin{array}{r}3586.480 \\
355 \\
3638.459 \\
355\end{array}$ & $\begin{array}{l}3586.453 \\
3638.486\end{array}$ & $\begin{array}{l}1.814 \\
1.827\end{array}$ & $\begin{array}{r}5.5368 \\
11\end{array}$ & 4.710 & $\begin{array}{l}0.1153 \\
0.1143\end{array}$ & $\begin{array}{l}2.2193 \\
2.2174\end{array}$ & $\begin{array}{l}-3.078 \\
-3.030\end{array}$ & $\begin{array}{l}6.0257 \\
(9 ; 17) \\
6.0262 \\
(11 ; 17)\end{array}$ & [Sh89] \\
\hline
\end{tabular}


G. FRICKE et al. Nuclear Charge Radii

TABLE IIIA. Muonic $2 p \rightarrow 1 s$ Transition Energies and Barrett Radii for $Z<60$ and $Z>77$ See page 194 for Explanation of Tables

\begin{tabular}{|c|c|c|c|c|c|c|c|c|c|c|}
\hline Isotope & $\begin{array}{c}E_{\text {exp. }} \\
{[\mathrm{keV}]}\end{array}$ & $\begin{array}{l}E_{\text {theo. }} \\
{[\mathrm{keV}]}\end{array}$ & $\begin{array}{l}\text { NPol } \\
\text { [keV] }\end{array}$ & $\begin{array}{c}c \\
{[\mathrm{fm}]}\end{array}$ & $\begin{array}{c}\left\langle r^{2}\right\rangle_{\text {model }}^{1 / 2} \\
{[\mathrm{fm}]}\end{array}$ & $\begin{array}{c}\alpha \\
{[1 / \mathrm{fm}]}\end{array}$ & $\mathbf{k}$ & $\begin{array}{c}C_{z} \\
{[\mathrm{am} / \mathrm{keV}]}\end{array}$ & $\begin{array}{l}R_{k \alpha}^{\mu} \\
{[\mathrm{fm}]}\end{array}$ & Ref. \\
\hline${ }^{123} \mathrm{Te}$ & $\begin{array}{r}3585.238 \\
38 \\
3637.191 \\
30\end{array}$ & $\begin{array}{l}3585.195 \\
3637.218\end{array}$ & $\begin{array}{l}1.635 \\
1.614\end{array}$ & $\begin{array}{r}5.5405 \\
1\end{array}$ & 4.712 & $\begin{array}{l}0.1153 \\
0.1143\end{array}$ & $\begin{array}{l}2.2193 \\
2.2174\end{array}$ & $\begin{array}{l}-3.081 \\
-3.033\end{array}$ & $\begin{array}{l}6.0290 \\
(1 ; 15) \\
6.0295 \\
(1 ; 15)\end{array}$ & [Sh89] \\
\hline${ }^{124} \mathrm{Te}$ & $\begin{array}{r}3582.489 \\
27 \\
3634.444 \\
24\end{array}$ & $\begin{array}{l}3582.481 \\
3634.451\end{array}$ & $\begin{array}{l}1.791 \\
1.777\end{array}$ & $\begin{array}{r}5.5503 \\
1\end{array}$ & 4.719 & $\begin{array}{l}0.1152 \\
0.1142\end{array}$ & $\begin{array}{l}2.2195 \\
2.2176\end{array}$ & $\begin{array}{l}-3.083 \\
-3.035\end{array}$ & $\begin{array}{l}6.0379 \\
(1 ; 16) \\
6.0384 \\
(1 ; 16)\end{array}$ & [Sh89] \\
\hline${ }^{125} \mathrm{Te}$ & $\begin{array}{r}3581.583 \\
34 \\
3633.531 \\
27\end{array}$ & $\begin{array}{l}3581.581 \\
3633.532\end{array}$ & $\begin{array}{l}1.580 \\
1.586\end{array}$ & $\begin{array}{r}5.5526 \\
1\end{array}$ & 4.721 & $\begin{array}{l}0.1152 \\
0.1142\end{array}$ & $\begin{array}{l}2.2196 \\
2.2177\end{array}$ & $\begin{array}{l}-3.084 \\
-3.036\end{array}$ & $\begin{array}{c}6.0401 \\
(1 ; 15) \\
6.0406 \\
(1 ; 15)\end{array}$ & [Sh89] \\
\hline${ }^{120} \mathrm{Te}$ & $\begin{array}{r}3578.997 \\
26 \\
3630.878 \\
23\end{array}$ & $\begin{array}{l}3578.967 \\
3630.901\end{array}$ & $\begin{array}{l}1.642 \\
1.664\end{array}$ & $\begin{array}{r}5.5617 \\
1\end{array}$ & 4.727 & $\begin{array}{l}0.1152 \\
0.1142\end{array}$ & $\begin{array}{l}2.2197 \\
2.2178\end{array}$ & $\begin{array}{l}-3.089 \\
-3.041\end{array}$ & $\begin{array}{l}6.0484 \\
(1 ; 15) \\
6.0489 \\
(1 ; 15)\end{array}$ & [Sh89] \\
\hline${ }^{128} \mathrm{Te}$ & $\begin{array}{r}3575.702 \\
30 \\
3627.576 \\
25\end{array}$ & $\begin{array}{l}3575.702 \\
3627.575\end{array}$ & $\begin{array}{l}1.617 \\
1.644\end{array}$ & $\begin{array}{r}5.5728 \\
1\end{array}$ & 4.735 & $\begin{array}{l}0.1151 \\
0.1141\end{array}$ & $\begin{array}{l}2.2199 \\
2.2180\end{array}$ & $\begin{array}{l}-3.092 \\
-3.044\end{array}$ & $\begin{array}{c}6.0585 \\
(1 ; 15) \\
6.0590 \\
(1 ; 15)\end{array}$ & [Sh89] \\
\hline${ }^{130} \mathrm{Te}$ & $\begin{array}{r}3572.642 \\
21 \\
3624.472 \\
18\end{array}$ & $\begin{array}{l}3572.644 \\
3624.471\end{array}$ & $\begin{array}{l}1.601 \\
1.628\end{array}$ & $\begin{array}{r}5.5832 \\
1\end{array}$ & 4.742 & $\begin{array}{l}0.1150 \\
0.1140\end{array}$ & $\begin{array}{l}2.2201 \\
2.2182\end{array}$ & $\begin{array}{l}-3.095 \\
-3.047\end{array}$ & $\begin{array}{l}6.0680 \\
(1 ; 15) \\
6.0685 \\
(1 ; 15)\end{array}$ & [Sh89] \\
\hline${ }^{127} J$ & $\begin{array}{r}3667.361 \\
35 \\
3723.742 \\
33\end{array}$ & $\begin{array}{l}3667.466 \\
3723.650\end{array}$ & $\begin{array}{l}0.532 \\
1.454\end{array}$ & $\begin{array}{r}5.5931 \\
1\end{array}$ & 4.749 & $\begin{array}{l}0.1166 \\
0.1155\end{array}$ & $\begin{array}{l}2.2229 \\
2.2209\end{array}$ & $\begin{array}{l}-2.969 \\
-2.919\end{array}$ & $\begin{array}{c}6.0762 \\
(1 ; 5) \\
6.0768 \\
(1 ; 13)\end{array}$ & [KI88] \\
\hline${ }^{124} X e$ & $\begin{array}{r}3761.655 \\
36 \\
3820.200 \\
27\end{array}$ & $\begin{array}{l}3761.572 \\
3820.251\end{array}$ & $\begin{array}{l}1.554 \\
1.423\end{array}$ & $\begin{array}{r}5.6114 \\
1\end{array}$ & 4.762 & $\begin{array}{l}0.1181 \\
0.1170\end{array}$ & $\begin{array}{l}2.2259 \\
2.2238\end{array}$ & $\begin{array}{l}-2.853 \\
-2.804\end{array}$ & $\begin{array}{l}6.0921 \\
(1 ; 13) \\
6.0927 \\
(1 ; 12)\end{array}$ & $\begin{array}{l}{[\mathrm{He} 85]} \\
{[\mathrm{He} 84]}\end{array}$ \\
\hline
\end{tabular}


TABLE IIIA. Muonic $2 p \rightarrow 1 s$ Transition Energies and Barrett Radii for $Z<60$ and $Z>77$ See page 194 for Explanation of Tables

\begin{tabular}{|c|c|c|c|c|c|c|c|c|c|c|}
\hline Isotope & $\begin{array}{c}E_{\text {exp. }} \\
{[\mathrm{keV}]}\end{array}$ & $\begin{array}{l}E_{\text {theo. }} \\
{[\mathrm{keV}]}\end{array}$ & $\begin{array}{l}\text { NPol } \\
{[\mathrm{keV}]}\end{array}$ & $\begin{array}{c}\mathrm{c} \\
{[\mathrm{fm}]}\end{array}$ & $\begin{array}{c}\left\langle r^{2}\right\rangle_{\text {model }}^{1 / 2} \\
{[\mathrm{fm}]}\end{array}$ & $\begin{array}{c}\alpha \\
{[1 / \mathrm{fm}]}\end{array}$ & k & $\begin{array}{c}C_{\mathrm{z}} \\
{[\mathrm{am} / \mathrm{keV}]}\end{array}$ & $\begin{array}{l}R_{k a}^{\mu} \\
{[\mathrm{fm}]}\end{array}$ & Ref. \\
\hline${ }^{138} X e$ & $\begin{array}{r}3757.900 \\
100 \\
3816.690 \\
100\end{array}$ & $\begin{array}{l}3757.961 \\
3816.630\end{array}$ & $\begin{array}{l}1.592 \\
1.517\end{array}$ & $\begin{array}{r}5.6229 \\
2\end{array}$ & 4.770 & $\begin{array}{l}0.1180 \\
0.1169\end{array}$ & $\begin{array}{l}2.2261 \\
2.2240\end{array}$ & $\begin{array}{l}-2.857 \\
-2.807\end{array}$ & $\begin{array}{c}6.1026 \\
(3 ; 14) \\
6.1032 \\
(3 ; 13)\end{array}$ & $\begin{array}{l}{[\mathrm{He} 85]} \\
{[\mathrm{He} 84]}\end{array}$ \\
\hline${ }^{128} X e$ & $\begin{array}{r}3755.586 \\
103 \\
3814.334 \\
103\end{array}$ & $\begin{array}{l}3755.626 \\
3814.295\end{array}$ & $\begin{array}{l}1.636 \\
1.603\end{array}$ & $\begin{array}{r}5.6304 \\
2\end{array}$ & 4.776 & $\begin{array}{l}0.1180 \\
0.1169\end{array}$ & $\begin{array}{l}2.2262 \\
2.2241\end{array}$ & $\begin{array}{l}-2.860 \\
-2.811\end{array}$ & $\begin{array}{c}6.1095 \\
(3 ; 14) \\
6.1100 \\
(3 ; 14)\end{array}$ & $\begin{array}{l}{[\mathrm{He} 85]} \\
{[\mathrm{He} 84]}\end{array}$ \\
\hline${ }^{129} \mathrm{Xe}$ & $\begin{array}{r}3755.135 \\
30 \\
3813.976 \\
27\end{array}$ & $\begin{array}{l}3755.165 \\
3813.952\end{array}$ & $\begin{array}{l}1.507 \\
1.598\end{array}$ & $\begin{array}{r}5.6315 \\
1\end{array}$ & 4.776 & $\begin{array}{l}0.1180 \\
0.1169\end{array}$ & $\begin{array}{l}2.2263 \\
2.2242\end{array}$ & $\begin{array}{l}-2.860 \\
-2.811\end{array}$ & $\begin{array}{l}6.1105 \\
(1 ; 13) \\
6.1110 \\
(1 ; 13)\end{array}$ & $\begin{array}{l}{[\mathrm{He} 85]} \\
{[\mathrm{He} 84]}\end{array}$ \\
\hline${ }^{130} X e$ & $\begin{array}{r}3752.245 \\
197 \\
3810.929 \\
197\end{array}$ & $\begin{array}{l}3752.272 \\
3810.903\end{array}$ & $\begin{array}{l}1.720 \\
1.711\end{array}$ & $\begin{array}{r}5.6412 \\
4\end{array}$ & 4.783 & $\begin{array}{l}0.1179 \\
0.1168\end{array}$ & $\begin{array}{l}2.2264 \\
2.2243\end{array}$ & $\begin{array}{l}-2.864 \\
-2.814\end{array}$ & $\begin{array}{c}6.1194 \\
(6 ; 15) \\
6.1200 \\
(6 ; 15)\end{array}$ & $\begin{array}{l}{[\mathrm{He} 85]} \\
{[\mathrm{He} 84]}\end{array}$ \\
\hline${ }^{131} \mathrm{Xe}$ & $\begin{array}{r}3753.060 \\
27 \\
3811.768 \\
24\end{array}$ & $\begin{array}{l}3753.078 \\
3811.754\end{array}$ & $\begin{array}{l}1.610 \\
1.629\end{array}$ & $\begin{array}{r}5.6384 \\
1\end{array}$ & 4.781 & $\begin{array}{l}0.1179 \\
0.1168\end{array}$ & $\begin{array}{l}2.2264 \\
2.2243\end{array}$ & $\begin{array}{l}-2.862 \\
-2.812\end{array}$ & $\begin{array}{l}6.1168 \\
(1 ; 14) \\
6.1174 \\
(1 ; 14)\end{array}$ & $\begin{array}{l}{[\mathrm{He} 85]} \\
{[\mathrm{He} 84]}\end{array}$ \\
\hline${ }^{132} X e$ & $\begin{array}{r}3750.737 \\
62 \\
3809.286 \\
42\end{array}$ & $\begin{array}{l}3750.677 \\
3809.313\end{array}$ & $\begin{array}{l}1.696 \\
1.719\end{array}$ & $\begin{array}{r}5.6460 \\
1\end{array}$ & 4.787 & $\begin{array}{l}0.1179 \\
0.1168\end{array}$ & $\begin{array}{l}2.2265 \\
2.2244\end{array}$ & $\begin{array}{l}-2.866 \\
-2.816\end{array}$ & $\begin{array}{l}6.1238 \\
(2 ; 15) \\
6.1244 \\
(1 ; 15)\end{array}$ & $\begin{array}{l}{[\mathrm{He} 85]} \\
{[\mathrm{He} 84]}\end{array}$ \\
\hline${ }^{134} X e$ & $\begin{array}{r}3748.217 \\
36 \\
3806.850 \\
27\end{array}$ & $\begin{array}{l}3748.238 \\
3806.838\end{array}$ & $\begin{array}{l}1.690 \\
1.722\end{array}$ & $\begin{array}{r}5.6539 \\
1\end{array}$ & 4.792 & $\begin{array}{l}0.1178 \\
0.1167\end{array}$ & $\begin{array}{l}2.2267 \\
2.2246\end{array}$ & $\begin{array}{l}-2.867 \\
-2.817\end{array}$ & $\begin{array}{l}6.1311 \\
(1 ; 15) \\
6.1316 \\
(1 ; 15)\end{array}$ & $\begin{array}{l}{[\mathrm{He} 85]} \\
{[\mathrm{He} 84]}\end{array}$ \\
\hline${ }^{136} \mathrm{Xe}$ & $\begin{array}{r}3744.956 \\
20 \\
3803.508 \\
18\end{array}$ & $\begin{array}{l}3744.960 \\
3803.505\end{array}$ & $\begin{array}{l}1.646 \\
1.681\end{array}$ & $\begin{array}{r}5.6641 \\
1\end{array}$ & 4.799 & $\begin{array}{l}0.1178 \\
0.1166\end{array}$ & $\begin{array}{l}2.2269 \\
2.2248\end{array}$ & $\begin{array}{l}-2.872 \\
-2.820\end{array}$ & $\begin{array}{l}6.1404 \\
(1 ; 14) \\
6.1410 \\
(1 ; 14)\end{array}$ & $\begin{array}{l}{[\mathrm{He} 85]} \\
{[\mathrm{He} 84]}\end{array}$ \\
\hline
\end{tabular}


G. FRICKE et al. Nuclear Charge Radii

TABLE IIIA. Muonic $2 p \rightarrow 1 s$ Transition Energies and Barrett Radii for $Z<60$ and $Z>77$ See page 194 for Explanation of Tables

\begin{tabular}{|c|c|c|c|c|c|c|c|c|c|c|}
\hline Isotope & $\begin{array}{c}E_{\text {exp }} \\
{[\mathrm{keV}]}\end{array}$ & $\begin{array}{l}E_{\text {theo. }} \\
{[\mathrm{keV}]}\end{array}$ & $\begin{array}{l}\text { NPol } \\
{[\mathrm{keV}]}\end{array}$ & $\begin{array}{c}c \\
{[\mathrm{fm}]}\end{array}$ & $\begin{array}{c}\left\langle r^{2}\right\rangle_{\text {model }}^{1 / 2} \\
{[\mathrm{fm}]}\end{array}$ & $\begin{array}{c}\alpha \\
{[1 / \mathrm{fm}]}\end{array}$ & k & $\begin{array}{c}C_{x} \\
{[\mathrm{am} / \mathrm{keV}]}\end{array}$ & $\begin{array}{l}R_{k \alpha}^{\mu} \\
{[\mathbf{f m}]}\end{array}$ & Ref. \\
\hline${ }^{133} \mathrm{Cs}$ & $\begin{array}{r}3840.702 \\
39 \\
3902.636 \\
31\end{array}$ & $\begin{array}{l}3840.670 \\
3902.656\end{array}$ & $\begin{array}{l}1.531 \\
1.289\end{array}$ & $\begin{array}{r}5.6710 \\
1\end{array}$ & 4.804 & $\begin{array}{l}0.1193 \\
0.1182\end{array}$ & $\begin{array}{l}2.2296 \\
2.2274\end{array}$ & $\begin{array}{l}-2.759 \\
-2.710\end{array}$ & $\begin{array}{c}6.1459 \\
(1 ; 13) \\
6.1464 \\
(1 ; 11)\end{array}$ & [K|88] \\
\hline${ }^{134} \mathrm{Ba}$ & $\begin{array}{r}3926.785 \\
31 \\
3992.683 \\
24\end{array}$ & $\begin{array}{l}3926.792 \\
3992.679\end{array}$ & $\begin{array}{l}1.757 \\
1.755\end{array}$ & $\begin{array}{r}5.7065 \\
1\end{array}$ & 4.829 & $\begin{array}{l}0.1207 \\
0.1195\end{array}$ & $\begin{array}{l}2.2328 \\
2.2305\end{array}$ & $\begin{array}{l}-2.665 \\
-2.614\end{array}$ & $\begin{array}{c}6.1776 \\
(1 ; 14) \\
6.1782 \\
(1 ; 14)\end{array}$ & $\begin{array}{l}\text { [Ku83] } \\
\text { [Sh82] }\end{array}$ \\
\hline${ }^{135} \mathrm{Ba}$ & $\begin{array}{r}3927.721 \\
75 \\
3993.742 \\
47\end{array}$ & $\begin{array}{l}3927.687 \\
3993.755\end{array}$ & $\begin{array}{l}1.548 \\
1.705\end{array}$ & $\begin{array}{r}5.7033 \\
1\end{array}$ & 4.827 & $\begin{array}{l}0.1207 \\
0.1195\end{array}$ & $\begin{array}{l}2.2327 \\
2.2305\end{array}$ & $\begin{array}{l}-2.663 \\
-2.612\end{array}$ & $\begin{array}{c}6.1747 \\
(2 ; 12) \\
6.1753 \\
(1 ; 13)\end{array}$ & [Sh82] \\
\hline${ }^{136} B a$ & $\begin{array}{r}3925.232 \\
25 \\
3991.093 \\
19\end{array}$ & $\begin{array}{l}3925.214 \\
3991.103\end{array}$ & $\begin{array}{l}1.756 \\
1.788\end{array}$ & $\begin{array}{r}5.7111 \\
1\end{array}$ & 4.833 & $\begin{array}{l}0.1207 \\
0.1194\end{array}$ & $\begin{array}{l}2.2329 \\
2.2306\end{array}$ & $\begin{array}{l}-2.667 \\
-2.614\end{array}$ & $\begin{array}{l}6.1819 \\
(1 ; 14) \\
6.1825 \\
(1 ; 14)\end{array}$ & $\begin{array}{l}\text { [Ku83] } \\
\text { [Sh82] }\end{array}$ \\
\hline${ }^{137} B a^{\star}$ & $\begin{array}{r}3991.390 \\
60\end{array}$ & 3991.390 & $\begin{array}{r}1.522 \\
1\end{array}$ & 5.7103 & 4.832 & 0.1194 & 2.2306 & -2.613 & $\begin{array}{l}6.1818 \\
(2 ; 12)\end{array}$ & [Sh82] \\
\hline${ }^{138} \mathrm{Ba}$ & $\begin{array}{r}3922.189 \\
15 \\
3987.988 \\
14\end{array}$ & $\begin{array}{l}3922.166 \\
3988.007\end{array}$ & $\begin{array}{l}1.737 \\
1.781\end{array}$ & $\begin{array}{r}5.7200 \\
1\end{array}$ & 4.839 & $\begin{array}{l}0.1206 \\
0.1194\end{array}$ & $\begin{array}{l}2.2331 \\
2.2308\end{array}$ & $\begin{array}{l}-2.669 \\
-2.618\end{array}$ & $\begin{array}{c}6.1900 \\
(1 ; 14) \\
6.1906 \\
(1 ; 14)\end{array}$ & $\begin{array}{l}{[\mathrm{Fr} 88 \mathrm{a}]} \\
{[\mathrm{Ku} 83]} \\
{[\mathrm{Sh} 82]}\end{array}$ \\
\hline${ }^{139} L a$ & $\begin{array}{r}4011.557 \\
40 \\
4081.408 \\
35\end{array}$ & $\begin{array}{l}4011.595 \\
4081.379\end{array}$ & $\begin{array}{l}1.626 \\
1.759\end{array}$ & $\begin{array}{r}5.7423 \\
1\end{array}$ & 4.855 & $\begin{array}{l}0.1220 \\
0.1208\end{array}$ & $\begin{array}{l}2.2360 \\
2.2336\end{array}$ & $\begin{array}{l}-2.575 \\
-2.524\end{array}$ & $\begin{array}{c}6.2097 \\
(1 ; 13) \\
6.2103 \\
(1 ; 13)\end{array}$ & {$[\operatorname{Re} 87]$} \\
\hline${ }^{140} \mathrm{Ce}$ & $\begin{array}{r}4097.389 \\
36 \\
4170.934 \\
34\end{array}$ & $\begin{array}{l}4097.361 \\
4170.959\end{array}$ & $\begin{array}{l}1.834 \\
1.885\end{array}$ & $\begin{array}{r}5.7739 \\
1\end{array}$ & 4.877 & $\begin{array}{l}0.1234 \\
0.1221\end{array}$ & $\begin{array}{l}2.2391 \\
2.2366\end{array}$ & $\begin{array}{l}-2.492 \\
-2.439\end{array}$ & $\begin{array}{c}6.2379 \\
(1 ; 14) \\
6.2385 \\
(1 ; 14)\end{array}$ & [Re87] \\
\hline
\end{tabular}

* The quoted experimental energy corresponds to the $2 p_{3 / 2} \rightarrow 1 s_{1 / 2}$ transition 
TABLE IIIA. Muonic $2 p \rightarrow 1 s$ Transition Energies and Barrett Radii for $Z<60$ and $Z>77$ See page 194 for Explanation of Tables

\begin{tabular}{|c|c|c|c|c|c|c|c|c|c|c|}
\hline Isotope & $\begin{array}{l}E_{\text {exp. }} \\
\text { [keV] }\end{array}$ & $\begin{array}{l}E_{\text {theo. }} \\
{[\mathrm{keV}]}\end{array}$ & $\begin{array}{l}\text { NPol } \\
{[\mathrm{keV}]}\end{array}$ & $\begin{array}{c}c \\
{[\mathrm{fm}]}\end{array}$ & $\begin{array}{c}\left\langle r^{2}\right)_{\text {model }}^{1 / 2} \\
{[\mathrm{fm}]}\end{array}$ & $\begin{array}{c}\alpha \\
{[1 / f m]}\end{array}$ & k & $\begin{array}{c}C_{x} \\
{[\mathrm{am} / \mathrm{keV}]}\end{array}$ & $\begin{array}{l}R_{k \alpha}^{\mu} \\
{[\mathrm{fm}]}\end{array}$ & Ref. \\
\hline${ }^{142} \mathrm{Ce}$ & $\begin{array}{r}4082.091 \\
127 \\
4155.245 \\
89\end{array}$ & $\begin{array}{l}4082.045 \\
4155.267\end{array}$ & $\begin{array}{l}1.739 \\
1.749\end{array}$ & $\begin{array}{r}5.815 .3 \\
2\end{array}$ & 4.907 & $\begin{array}{l}0.1231 \\
0.1218\end{array}$ & $\begin{array}{l}2.2399 \\
2.2374\end{array}$ & $\begin{array}{l}-2.505 \\
-2.452\end{array}$ & $\begin{array}{c}6.2760 \\
(3 ; 13) \\
6.2767 \\
(2 ; 13)\end{array}$ & [Re87] \\
\hline${ }^{141} \mathrm{Pr}$ & $\begin{array}{r}4185.908 \\
27 \\
4263.595 \\
27\end{array}$ & $\begin{array}{l}4185.905 \\
4263.598\end{array}$ & $\begin{array}{l}1.726 \\
1.784\end{array}$ & $\begin{array}{r}5.7950 \\
1\end{array}$ & 4.892 & $\begin{array}{l}0.1248 \\
0.1234\end{array}$ & $\begin{array}{l}2.2420 \\
2.2394\end{array}$ & $\begin{array}{l}-2.409 \\
-2.355\end{array}$ & $\begin{array}{c}6.2565 \\
(1 ; 12) \\
6.2572 \\
(1 ; 13)\end{array}$ & [Re87] \\
\hline${ }^{142} N d^{\dagger}$ & $\begin{array}{r}4352.354 \\
51\end{array}$ & & 1.957 & 5.8249 & 4.914 & 0.1244 & 2.2433 & -2.280 & $\begin{array}{c}6.2842 \\
(1 ; 13)\end{array}$ & {$[\operatorname{Re} 87]$} \\
\hline${ }^{143} N d^{\dagger}$ & $\begin{array}{r}4346.261 \\
148\end{array}$ & & 1.962 & 5.8400 & 4.924 & 0.1244 & 2.2433 & -2.280 & $\begin{array}{l}6.2981 \\
(3 ; 13)\end{array}$ & [Re87] \\
\hline${ }^{144} N d^{\dagger}$ & $\begin{array}{r}4336.628 \\
58\end{array}$ & & 1.799 & 5.86336 & 4.941 & 0.1244 & 2.24333 & -2.290 & $\begin{array}{l}6.3197 \\
(1 ; 12)\end{array}$ & [Re87] \\
\hline${ }^{145} N d^{\dagger}$ & $\begin{array}{r}43330.555 \\
152\end{array}$ & & 2.459 & 5.8805 & 4.953 & 0.1244 & 2.2433 & -2.300 & $\begin{array}{c}6.3351 \\
(3 ; 17)\end{array}$ & [Re87] \\
\hline${ }^{146} \mathrm{Nd}{ }^{\dagger}$ & $\begin{array}{r}4321.108 \\
61\end{array}$ & & 1.492 & 5.9017 & 4.968 & 0.1244 & 2.2433 & -2.310 & $\begin{array}{c}6.3546 \\
(1 ; 10)\end{array}$ & [Re87] \\
\hline${ }^{148} N d^{\dagger}$ & $\begin{array}{r}4303.519 \\
73\end{array}$ & & 0.566 & $5.94: 36$ & 4.998 & 0.1244 & 2.2433 & -2.320 & $\begin{array}{c}6.3930 \\
(2 ; 4)\end{array}$ & {$[\operatorname{Re} 87]$} \\
\hline
\end{tabular}

$\dagger$ The quoted energy corresponds to the $2 p_{3 / 2} \rightarrow 1 s_{1 / 2}$ transition. All data are taken from the quoted reference. 
G. FRICKE et al. Nuclear Charge Radii

TABLE IIIA. Muonic $2 p \rightarrow 1 s$ Transition Energies and Barrett Radii for $Z<60$ and $Z>77$ See page 194 for Explanation of Tables

\begin{tabular}{|c|c|c|c|c|c|c|c|c|c|c|}
\hline Isotope & $\begin{array}{c}E_{\text {exp. }} \\
{[\mathrm{keV}]}\end{array}$ & $\begin{array}{l}E_{\text {theo. }} \\
\text { [keV] }\end{array}$ & $\begin{array}{l}\text { NPol } \\
\text { [keV] }\end{array}$ & $\begin{array}{c}\mathrm{c} \\
{[\mathrm{fm}]}\end{array}$ & $\begin{array}{c}\left\langle r^{2}\right\rangle_{\text {model }}^{1 / 2} \\
{[\mathrm{fm}]}\end{array}$ & $\begin{array}{c}\alpha \\
{[1 / \mathrm{fm}]}\end{array}$ & $\mathbf{k}$ & $\begin{array}{c}C_{x} \\
{[\mathrm{am} / \mathrm{keV}]}\end{array}$ & $\begin{array}{l}R_{k \alpha}^{\mu} \\
{[\mathrm{fm}]}\end{array}$ & Ref. \\
\hline${ }^{194} P t$ & $\begin{array}{r}5520.321 \\
153 \\
5680.145 \\
163\end{array}$ & $\begin{array}{l}5520.342 \\
5680.123\end{array}$ & $\begin{array}{l}-0.700 \\
-5.151\end{array}$ & $\begin{array}{r}6.5370 \\
2\end{array}$ & 5.425 & $\begin{array}{l}0.1465 \\
0.1443\end{array}$ & $\begin{array}{l}2.2981 \\
2.2943\end{array}$ & $\begin{array}{l}-1.576 \\
-1.506\end{array}$ & $\begin{array}{l}6.9305 \\
(2 ; 23) \\
6.9317 \\
(2 ; 23)\end{array}$ & [Be90] \\
\hline${ }^{105} \mathrm{Pt}$ & $\begin{array}{r}5517.956 \\
214 \\
5677.304 \\
222\end{array}$ & $\begin{array}{l}5518.092 \\
5677.164\end{array}$ & $\begin{array}{l}-0.778 \\
-5.839\end{array}$ & $\begin{array}{r}6.5407 \\
2\end{array}$ & 5.427 & $\begin{array}{l}0.1465 \\
0.1443\end{array}$ & $\begin{array}{l}2.2982 \\
2.2944\end{array}$ & $\begin{array}{l}-1.577 \\
-1.508\end{array}$ & $\begin{array}{l}6.9339 \\
(3 ; 23) \\
6.9352 \\
(3 ; 23)\end{array}$ & [Be90] \\
\hline${ }^{190} P t$ & $\begin{array}{r}5514.916 \\
198 \\
5675.898 \\
206\end{array}$ & $\begin{array}{l}5514.918 \\
5675.896\end{array}$ & $\begin{array}{l}-0.125 \\
-3.105\end{array}$ & $\begin{array}{r}6.5472 \\
1\end{array}$ & 5.432 & $\begin{array}{l}0.1464 \\
0.1443\end{array}$ & $\begin{array}{l}2.2983 \\
2.2945\end{array}$ & $\begin{array}{l}-1.578 \\
-1.510\end{array}$ & $\begin{array}{c}6.9400 \\
(3 ; 18) \\
6.9412 \\
(3 ; 18)\end{array}$ & [Be90] \\
\hline${ }^{108} \mathrm{Pt}$ & $\begin{array}{r}5507.857 \\
246 \\
5670.357 \\
252\end{array}$ & $\begin{array}{l}5508.135 \\
5670.078\end{array}$ & $\begin{array}{r}0.506 \\
-1.170\end{array}$ & $\begin{array}{r}6.5597 \\
1\end{array}$ & 5.441 & $\begin{array}{l}0.1463 \\
0.1442\end{array}$ & $\begin{array}{l}2.2986 \\
2.2947\end{array}$ & $\begin{array}{l}-1.581 \\
-1.513\end{array}$ & $\begin{array}{c}6.9518 \\
(4 ; 5) \\
6.9530 \\
(4 ; 5)\end{array}$ & [Be90] \\
\hline${ }^{107} \mathrm{Au}$ & $\begin{array}{r}5591.710 \\
146 \\
5760.792 \\
153\end{array}$ & $\begin{array}{l}5591.790 \\
5760.708\end{array}$ & $\begin{array}{l}-0.538 \\
-1.305\end{array}$ & $\begin{array}{r}6.5541 \\
1\end{array}$ & 5.437 & $\begin{array}{l}0.1477 \\
0.1455\end{array}$ & $\begin{array}{l}2.3004 \\
2.2965\end{array}$ & $\begin{array}{l}-1.546 \\
-1.477\end{array}$ & $\begin{array}{c}6.9457 \\
(2 ; 6) \\
6.9469 \\
(2 ; 6)\end{array}$ & {$[\mathrm{Be} 90]$} \\
\hline${ }^{108} \mathrm{Hg}^{\star}$ & $\begin{array}{r}5664.540 \\
255 \\
5838.270 \\
256\end{array}$ & $\begin{array}{l}5664.667 \\
5838.148\end{array}$ & $\begin{array}{r}0.593 \\
-1.174\end{array}$ & $\begin{array}{r}6.5691 \\
1\end{array}$ & 5.448 & $\begin{array}{l}0.1488 \\
0.1466\end{array}$ & $\begin{array}{l}2.3025 \\
2.2986\end{array}$ & $\begin{array}{l}-1.516 \\
-1.447\end{array}$ & $\begin{array}{c}6.9590 \\
(4 ; 6) \\
6.9603 \\
(4 ; 6)\end{array}$ & $\begin{array}{l}\text { [Bu89] } \\
\text { [Ha79] }\end{array}$ \\
\hline${ }^{100} \mathrm{Hg}^{\star}$ & $\begin{array}{r}5663.150 \\
540 \\
5839.480 \\
460\end{array}$ & $\begin{array}{l}5662.815 \\
5840.405\end{array}$ & $\begin{array}{r}-1.254 \\
2.137\end{array}$ & $\begin{array}{r}6.5708 \\
4\end{array}$ & 5.449 & $\begin{array}{l}0.1488 \\
0.1466\end{array}$ & $\begin{array}{l}2.3026 \\
2.2986\end{array}$ & $\begin{array}{l}-1.517 \\
-1.448\end{array}$ & $\begin{array}{c}6.9598 \\
(8 ; 9) \\
6.9612 \\
(7 ; 9)\end{array}$ & $\begin{array}{l}\text { [Gu83] } \\
{[\mathrm{Ha} 79]}\end{array}$ \\
\hline${ }^{200} \mathrm{Hg}^{\star}$ & $\begin{array}{r}5656.360 \\
272 \\
5829.690 \\
255\end{array}$ & $\begin{array}{l}5656.618 \\
5829.474\end{array}$ & $\begin{array}{r}0.694 \\
-1.305\end{array}$ & $\begin{array}{r}6.5824 \\
3\end{array}$ & 5.457 & $\begin{array}{l}0.1487 \\
0.1465\end{array}$ & $\begin{array}{l}2.3028 \\
2.2989\end{array}$ & $\begin{array}{l}-1.520 \\
-1.450\end{array}$ & $\begin{array}{c}6.9714 \\
(4 ; 6) \\
6.9727 \\
(4 ; 6)\end{array}$ & $\begin{array}{l}{[\mathrm{Bu} 89]} \\
{[\mathrm{Gu} 83]} \\
{[\mathrm{Ha} 79]}\end{array}$ \\
\hline
\end{tabular}

* Energies are taken from the first quoted reference 
TABLE IIIA. Muonic $2 p \rightarrow 1 s$ Transition Energies and Barrett Radii for $Z<60$ and $Z>77$ See page 194 for Explanation of Tables

\begin{tabular}{|c|c|c|c|c|c|c|c|c|c|c|}
\hline Isotope & $\begin{array}{l}E_{\text {exp. }} \\
{[\mathrm{keV}]}\end{array}$ & $\begin{array}{l}E_{\text {theo. }} \\
{[\mathrm{keV}]}\end{array}$ & $\begin{array}{l}\text { NPol } \\
{[\mathrm{keV}]}\end{array}$ & $\begin{array}{c}c \\
{[\mathrm{fm}]}\end{array}$ & $\begin{array}{c}\left\langle r^{2}\right\rangle_{\text {model }}^{1 / 2} \\
{[\mathrm{fm}]}\end{array}$ & $\begin{array}{c}\alpha \\
{[1 / f m]}\end{array}$ & $\mathbf{k}$ & $\begin{array}{c}C_{\mathrm{z}} \\
{[\mathrm{am} / \mathrm{keV}]}\end{array}$ & $\begin{array}{l}R_{k \alpha}^{\mu} \\
{[\mathrm{fm}]}\end{array}$ & Ref. \\
\hline${ }^{202} H g^{\star}$ & $\begin{array}{r}5649.350 \\
327 \\
5822.550 \\
255\end{array}$ & $\begin{array}{l}5649.103 \\
5822.693\end{array}$ & $\begin{array}{l}1.240 \\
0.364\end{array}$ & $\begin{array}{r}6.5955 \\
3\end{array}$ & 5.467 & $\begin{array}{l}0.1486 \\
0.1464\end{array}$ & $\begin{array}{l}2.3030 \\
2.2991\end{array}$ & $\begin{array}{l}-1.523 \\
-1.454\end{array}$ & $\begin{array}{c}6.9838 \\
(5 ; 6) \\
6.9850 \\
(4 ; 6)\end{array}$ & $\begin{array}{l}\text { [Bu89] } \\
{[\mathrm{Ha} 79]}\end{array}$ \\
\hline${ }^{204} \mathrm{Hg}^{\star}$ & $\begin{array}{r}5641.600 \\
825 \\
5814.420 \\
380\end{array}$ & $\begin{array}{l}5640.627 \\
5814.617\end{array}$ & $\begin{array}{l}2.069 \\
2.040\end{array}$ & $\begin{array}{r}6.6107 \\
6\end{array}$ & 5.478 & $\begin{array}{l}0.1485 \\
0.1463\end{array}$ & $\begin{array}{l}2.3033 \\
2.2994\end{array}$ & $\begin{array}{l}-1.528 \\
-1.458\end{array}$ & $\begin{array}{c}6.9980 \\
(13 ; 10) \\
6.9993 \\
(6 ; 9)\end{array}$ & $\begin{array}{l}{[\text { Bu89] }} \\
{[\mathrm{Ha79}]}\end{array}$ \\
\hline${ }^{203} \mathrm{Tl}^{\dagger}$ & $\begin{array}{r}5726.140 \\
670 \\
5906.380 \\
670\end{array}$ & $\begin{array}{l}5727.210 \\
5907.500\end{array}$ & $\begin{array}{l}3.737 \\
3.737\end{array}$ & $\begin{array}{r}6.6023 \\
6\end{array}$ & 5.472 & $\begin{array}{l}0.1520 \\
0.1520\end{array}$ & $\begin{array}{l}2.3170 \\
2.3270\end{array}$ & $\begin{array}{l}-1.496 \\
-1.426\end{array}$ & $\begin{array}{l}6.9903 \\
(10 ; 19) \\
6.9911 \\
(10 ; 19)\end{array}$ & $\begin{array}{l}{[\text { En74] }} \\
{[\mathrm{Ba72}]}\end{array}$ \\
\hline${ }^{205} \mathrm{Tl}^{\dagger}$ & $\begin{array}{r}5717.210 \\
650 \\
5897.290 \\
670\end{array}$ & $\begin{array}{l}5717.870 \\
5897.690\end{array}$ & $\begin{array}{l}3.737 \\
3.737\end{array}$ & $\begin{array}{r}6.6173 \\
6\end{array}$ & $5.48: 3$ & $\begin{array}{l}0.1520 \\
0.1520\end{array}$ & $\begin{array}{l}2.3180 \\
2.3280\end{array}$ & $\begin{array}{l}-1.500 \\
-1.429\end{array}$ & $\begin{array}{l}7.0037 \\
(10 ; 19) \\
7.0042 \\
(10 ; 19)\end{array}$ & $\begin{array}{l}{[\text { En74] }} \\
{[\text { Ba72] }}\end{array}$ \\
\hline${ }^{204} \mathrm{~Pb}^{\ddagger}$ & $\begin{array}{r}5796.318 \\
180 \\
5982.124 \\
158\end{array}$ & $\begin{array}{l}5796.205 \\
5982.207\end{array}$ & $\begin{array}{l}2.086 \\
2.136\end{array}$ & $\begin{array}{r}6.6169 \\
2\end{array}$ & 5.482 & $\begin{array}{l}0.1510 \\
0.1487\end{array}$ & $\begin{array}{l}2.3071 \\
2.3031\end{array}$ & $\begin{array}{l}-1.467 \\
-1.396\end{array}$ & $\begin{array}{c}7.0022 \\
(3 ; 9) \\
7.00 ; 36 \\
(3 ; 9)\end{array}$ & $\begin{array}{l}{[\mathrm{Ke} 75]} \\
{[\mathrm{Be} 88]}\end{array}$ \\
\hline${ }^{200} \mathrm{~Pb}^{\ddagger}$ & $\begin{array}{r}5787.208 \\
172 \\
5972.794 \\
158\end{array}$ & $\begin{array}{l}5787.219 \\
5972.785\end{array}$ & $\begin{array}{l}2.129 \\
2.201\end{array}$ & $\begin{array}{r}6.6311 \\
2\end{array}$ & 5.493 & $\begin{array}{l}0.1509 \\
0.1486\end{array}$ & $\begin{array}{l}2.3073 \\
2.3034\end{array}$ & $\begin{array}{l}-1.471 \\
-1.400\end{array}$ & $\begin{array}{c}7.0155 \\
(3 ; 9) \\
7.0169 \\
(2 ; 9)\end{array}$ & $\begin{array}{l}{[\mathrm{Ke} 75]} \\
{[\mathrm{Be} 88]}\end{array}$ \\
\hline${ }^{207} \mathrm{~Pb} \ddagger$ & $\begin{array}{r}5783.988 \\
215 \\
5968.864 \\
210\end{array}$ & $\begin{array}{r}5783.709 \\
5969.117\end{array}$ & $\begin{array}{l}2.209 \\
2.304\end{array}$ & $\begin{array}{r}6.6367 \\
2\end{array}$ & 5.497 & $\begin{array}{l}0.1508 \\
0.1486\end{array}$ & $\begin{array}{l}2.3074 \\
2.3035\end{array}$ & $\begin{array}{l}-1.472 \\
-1.402\end{array}$ & $\begin{array}{c}7.0209 \\
(3 ; 10) \\
7.0222 \\
(3 ; 10)\end{array}$ & $\begin{array}{l}\text { [Ke75] } \\
\text { [Be88] }\end{array}$ \\
\hline${ }^{208} \mathrm{~Pb}$ & $\begin{array}{r}5778.058 \\
100 \\
5962.854 \\
90\end{array}$ & $\begin{array}{l}5778.076 \\
5962.840\end{array}$ & $\begin{array}{l}2.945 \\
2.718\end{array}$ & $\begin{array}{r}6.6468 \\
1\end{array}$ & 5.504 & $\begin{array}{l}0.1507 \\
0.1485\end{array}$ & $\begin{array}{l}2.3076 \\
2.3037\end{array}$ & $\begin{array}{l}-1.474 \\
-1.404\end{array}$ & $\begin{array}{c}7.0303 \\
(2 ; 13) \\
7.0316 \\
(1 ; 11)\end{array}$ & [Be88] \\
\hline
\end{tabular}

* Energies are taken from the first quoted reference

$\dagger$ Here, all data are taken from the quoted reference

$\ddagger$ Energies are based on $E\left({ }^{208} \mathrm{~Pb}\right)[\mathrm{Be} 88]$ and $\Delta E[\mathrm{Ke} 75]$ 
TABLE IIIA. Muonic $2 p \rightarrow 1 s$ Transition Energies and Barrett Radii for $Z<60$ and $Z>77$ See page 194 for Explanation of Tables

\begin{tabular}{|c|c|c|c|c|c|c|c|c|c|c|}
\hline Isotope & $\begin{array}{l}E_{\text {exp. }} \\
{[\text { keV] }}\end{array}$ & $\begin{array}{l}E_{\text {theo. }} \\
\text { [keV] }\end{array}$ & $\begin{array}{l}\text { NPol } \\
{[\mathrm{keV}]}\end{array}$ & $\begin{array}{c}c \\
{[\mathrm{fm}]}\end{array}$ & $\begin{array}{c}\left\langle r^{2}\right\rangle_{\text {model }}^{1 / 2} \\
{[\mathrm{fm}]}\end{array}$ & $\begin{array}{c}\alpha \\
{[1 / f \mathrm{fm}]}\end{array}$ & k & $\begin{array}{c}C_{*} \\
{[\mathrm{am} / \mathrm{keV}]}\end{array}$ & $\begin{array}{l}R_{k \alpha}^{\mu} \\
{[\mathrm{fm}]}\end{array}$ & Ref. \\
\hline${ }^{200} \mathrm{Bi}^{\dagger}$ & $\begin{array}{r}5843.200 \\
2.500 \\
6034.000 \\
2.200\end{array}$ & & $\begin{array}{l}3.844 \\
3.844\end{array}$ & $\begin{array}{r}6.6869 \\
11\end{array}$ & 5.533 & $\begin{array}{l}0.1540 \\
0.1540\end{array}$ & $\begin{array}{l}2.329 \\
2.339\end{array}$ & $\begin{array}{l}-1.458 \\
-1.386\end{array}$ & $\begin{array}{c}7.0501 \\
(36 ; 18) \\
7.0504 \\
(30 ; 18)\end{array}$ & $\begin{array}{c}{[\mathrm{En74}]} \\
{[\mathrm{Po68}]} \\
{[\mathrm{Ru} 84 \mathrm{~b}]}\end{array}$ \\
\hline $\begin{array}{r}233,25 \\
230\end{array}$ & $\begin{array}{r}{ }^{232} \mathrm{Th} \\
{ }^{4,235,238} \mathrm{U} \\
{ }_{240,212} \mathrm{Pu}\end{array}$ & & & & & & & & & $\begin{array}{l}{[\mathrm{Zu} 86]} \\
{[\mathrm{Zu} 84]} \\
{[\mathrm{Zu} 86]}\end{array}$ \\
\hline
\end{tabular}

$\dagger$ Here, all data are taken from the quoted reference 
G. FRICKE et al, Nuclear Charge Radii

TABLE IIIB. Muonic $2 p \rightarrow 1 s$ Transition Energies and Relative Intensities of Deformed Nuclei with $60 \leqslant Z \leqslant 77$

See page 194 for Explanation of Tables

\begin{tabular}{|c|c|c|c|c|c|}
\hline \multirow[t]{2}{*}{ Isotope } & \multicolumn{2}{|c|}{ Transition } & \multicolumn{2}{|c|}{ Energy [keV] } & \multirow{2}{*}{$\begin{array}{l}\text { Relative } \\
\text { Intensity }\end{array}$} \\
\hline & Initial state & Final state & $E_{\text {exp }}$ & $E_{\text {theo }}$ & \\
\hline \multirow{9}{*}{${ }_{60}^{150} \mathrm{Nd}$} & $\left|2 p_{1 / 2} \otimes 0^{+} ; \frac{1}{2}^{-}\right\rangle$ & $\left|1 s_{1 / 2} \otimes 0^{+} ; \frac{1}{2}^{+}\right\rangle$ & $4198.118(55)$ & 4198.086 & 0.951 \\
\hline & $\left|2 p_{1 / 2} \otimes 2^{+} ; \frac{3}{2}^{-}\right\rangle$ & $\left|1 s_{1 / 2} \otimes 2^{+} ; \frac{5}{2}^{+}\right\rangle$ & $4212.492(55)$ & 4212.460 & 0.218 \\
\hline & $\left|2 p_{1 / 2} \otimes 2^{+} ; \frac{3}{2}^{-}\right\rangle$ & $\left|1 s_{1 / 2} \otimes 0^{+} ;^{+}{ }^{+}\right\rangle$ & $4343.963(55)$ & 4343.926 & 0.068 \\
\hline & $\left|2 p_{1 / 2} \otimes 0^{+} ; \frac{1}{2}^{-}\right\rangle$ & $\left|1 s_{1 / 2} \otimes 2^{+} ; \frac{3}{2}^{+}\right\rangle$ & $4067.077(55)$ & 4067.046 & 0.033 \\
\hline & $\left|2 p_{1 / 2} \otimes 2^{+} ; \frac{3}{2}^{-}\right\rangle$ & $\left|1 s_{1 / 2} \otimes 2^{+} ;_{\frac{3}{2}}^{+}\right\rangle$ & $4212.918(55)$ & 4212.886 & 0.018 \\
\hline & $\left|2 p_{3 / 2} \otimes 0^{+} ; \frac{3}{2}^{-}\right\rangle$ & $\left|1 s_{1 / 2} \otimes 0^{+} ; \frac{1}{2}^{+}\right\rangle$ & $4266.573(52)$ & 4266.593 & 1.000 \\
\hline & $\left|2 p_{3 / 2} \otimes 0^{+} ; \frac{3}{2}^{-}\right\rangle$ & $\left|1 s_{1 / 2} \otimes 2^{+} ; \frac{5}{2}^{+}\right\rangle$ & $4135.104(52)$ & 4135.127 & 0.259 \\
\hline & $\left|2 p_{3 / 2} \otimes 2^{+} ; \frac{1}{2}^{-}\right\rangle$ & $\left.\mid 1 s_{1 / 2} \otimes 2^{+} ; \frac{3}{2}^{+}\right\}$ & $4265.684(52)$ & 4265.704 & 0.048 \\
\hline & $\left|2 p_{3 / 2} \otimes 2^{+} ; \frac{3}{2}^{-}\right\rangle$ & $\left.\mid 1 s_{1 / 2} \otimes 2^{+} ; \frac{3}{2}^{+}\right\}$ & $4294.264(52)$ & 4294.284 & 0.038 \\
\hline
\end{tabular}

\begin{tabular}{|c|c|c|c|c|c|}
\hline${ }_{84}^{155} \mathrm{Gd}$ & $\begin{array}{l}\left|2 p_{1 / 2} \otimes \frac{3}{2}^{-} ; 2^{+}\right\rangle \\
\left|2 p_{1 / 2} \otimes \frac{3}{2}^{-} ; 1^{+}\right\rangle \\
\left|2 p_{1 / 2} \otimes \frac{3}{2}^{-} ; 1^{+}\right\rangle \\
\left|2 p_{1 / 2} \otimes \frac{3}{2} ; 2^{+}\right\rangle \\
\left|2 p_{1 / 2} \otimes \frac{5}{2} ; 2^{+}\right\rangle \\
\left|2 p_{1 / 2} \otimes \frac{5}{2} ; 2^{+}\right\rangle \\
\left|2 p_{1 / 2} \otimes \frac{5}{2}^{-} ; 3^{+}\right\rangle \\
\left|2 p_{3 / 2} \otimes \frac{3}{2}^{-} ; 1^{+}\right\rangle \\
\left|2 p_{3 / 2} \otimes \frac{3}{2}^{-} ; 3^{+}\right\rangle \\
\left|2 p_{3 / 2} \otimes \frac{3}{2} ; 3^{+}\right\rangle \\
\left|2 p_{3 / 2} \otimes \frac{3}{2}^{-} ; 1^{+}\right\rangle \\
\left|2 p_{3 / 2} \otimes \frac{3}{2}^{-} ; 2^{+}\right\rangle \\
\left|2 p_{3 / 2} \otimes \frac{3}{2}^{-} ; 0^{+}\right\rangle \\
\left|2 p_{3 / 2} \otimes \frac{3}{2}^{-} ; 3^{+}\right\rangle \\
\left|2 p_{3 / 2} \otimes \frac{3}{2}^{-} ; 3^{+}\right\rangle \\
\left|2 p_{3 / 2} \otimes \frac{3}{2}-3^{+}\right\rangle \\
\left|2 p_{3 / 2} \otimes \frac{3}{2}^{-} ; 2^{+}\right\rangle \\
\left|2 p_{3 / 2} \otimes \frac{3}{2}^{-} ; 2^{+}\right\rangle \\
\left|2 p_{3 / 2} \otimes \frac{3}{2}^{-} ; 3^{+}\right\rangle \\
\left|2 p_{3 / 2} \otimes \frac{3}{2}^{-} ; 2^{+}\right\rangle\end{array}$ & $\begin{array}{l}\left|1 s_{1 / 2} \otimes \frac{3}{2}^{-} ; 2^{-}\right\rangle \\
\left|1 s_{1 / 2} \otimes \frac{3}{2}^{-} ; 2^{-}\right\rangle \\
\left|1 s_{1 / 2} \otimes \frac{3}{2}^{-} ; 1^{-}\right\rangle \\
\left|1 s_{1 / 2} \otimes \frac{3}{2}^{-} ; 1^{-}\right\rangle \\
\left|1 s_{1 / 2} \otimes \frac{5}{2}^{-} ; 3^{-}\right\rangle \\
\left|1 s_{1 / 2} \otimes \frac{3}{2}^{-} ; 1^{-}\right\rangle \\
\left|1 s_{1 / 2} \otimes \frac{5}{2}^{-} ; 3^{-}\right\rangle \\
\left|1 s_{1 / 2} \otimes \frac{3}{2}^{-} ; 2^{-}\right\rangle \\
\left|1 s_{1 / 2} \otimes \frac{3}{2}^{-} ; 2^{-}\right\rangle \\
\left|1 s_{1 / 2} \otimes \frac{3}{2}^{-} ; 2^{-}\right\rangle \\
\left|1 s_{1 / 2} \otimes \frac{3}{2}^{-} ; 1^{-}\right\rangle \\
\left|1 s_{1 / 2} \otimes \frac{3}{2}^{-} ; 1^{-}\right\rangle \\
\left|1 s_{1 / 2} \otimes \frac{3}{2}-; 2^{-}\right\rangle \\
\left|1 s_{1 / 2} \otimes \frac{5}{2}^{-} ; 2^{-}\right\rangle \\
\left|1 s_{1 / 2} \otimes \frac{7}{2}^{-} ; 4^{-}\right\rangle \\
\left|1 s_{1 / 2} \otimes \frac{7}{2}^{-} ; 4^{-}\right\rangle \\
\left|1 s_{1 / 2} \otimes \frac{5}{2}^{-} ; 3^{-}\right\rangle \\
\left|1 s_{1 / 2} \otimes \frac{5}{2}^{-} ; 2^{-}\right\rangle \\
\left|1 s_{1 / 2} \otimes \frac{5}{2}^{-} ; 3^{-}\right\rangle \\
\left|1 s_{1 / 2} \otimes \frac{3}{2}^{-} ; 2^{-}\right\rangle\end{array}$ & $\begin{array}{l}4510.809(116) \\
4505.706(116) \\
4505.469(116) \\
4510.572(116) \\
4487.347(116) \\
4547.267(116) \\
4510.635(116) \\
4633.141(108) \\
4599.782(108) \\
4679.117(108) \\
4632.904(108) \\
46339.526(108) \\
4660.116(108) \\
4539.616(108) \\
4532.977(108) \\
4453.640(108) \\
4579.605(108) \\
4579.597(108) \\
4618.958(108) \\
4639.762(108)\end{array}$ & $\begin{array}{l}4633.150 \\
4599.791 \\
4679.124 \\
4632.914 \\
4639.534 \\
4660.125 \\
4539.627 \\
4532.990 \\
4453.656 \\
4579.608 \\
4579.615 \\
4618.961 \\
4639.762\end{array}$ & $\begin{array}{l}1.000 \\
0.449 \\
0.389 \\
0.359 \\
0.329 \\
0.246 \\
0.151 \\
\\
0.403 \\
0.399 \\
0.380 \\
0.333 \\
0.331 \\
0.302 \\
0.300 \\
0.225 \\
0.187 \\
0.178 \\
0.154 \\
0.148 \\
0.146\end{array}$ \\
\hline
\end{tabular}


TABLE IIIB. Muonic $2 p \rightarrow 1 s$ Transition Energies and Relative Intensities of Deformed Nuclei with $60 \leqslant Z \leqslant 77$

See page 194 for Explanation of Tables

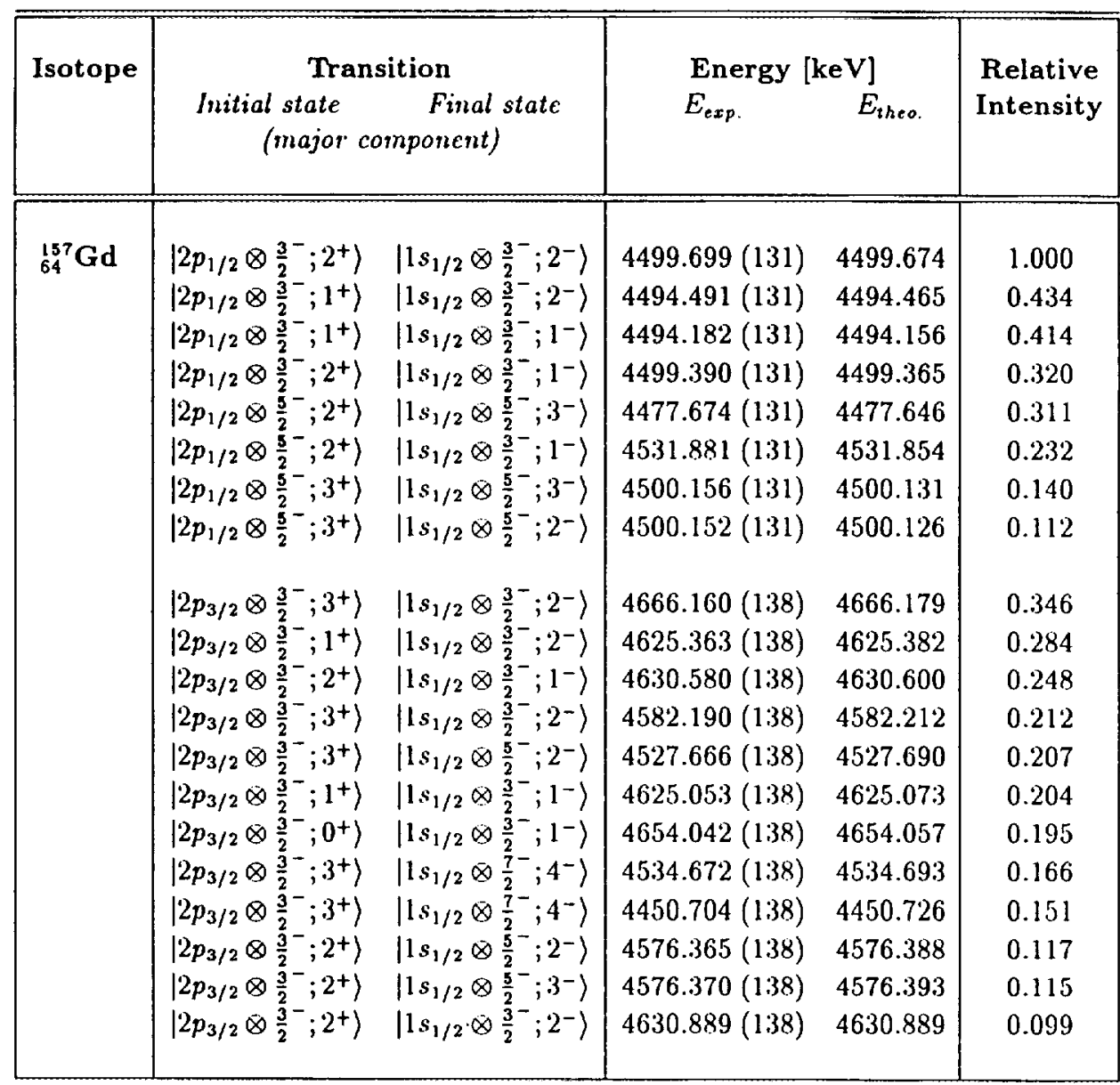


TABLE IIIB. Muonic $2 p \rightarrow 1 s$ Transition Energies and Relative Intensities of Deformed Nuclei with $60 \leqslant Z \leqslant 77$

See page 194 for Explanation of Tables

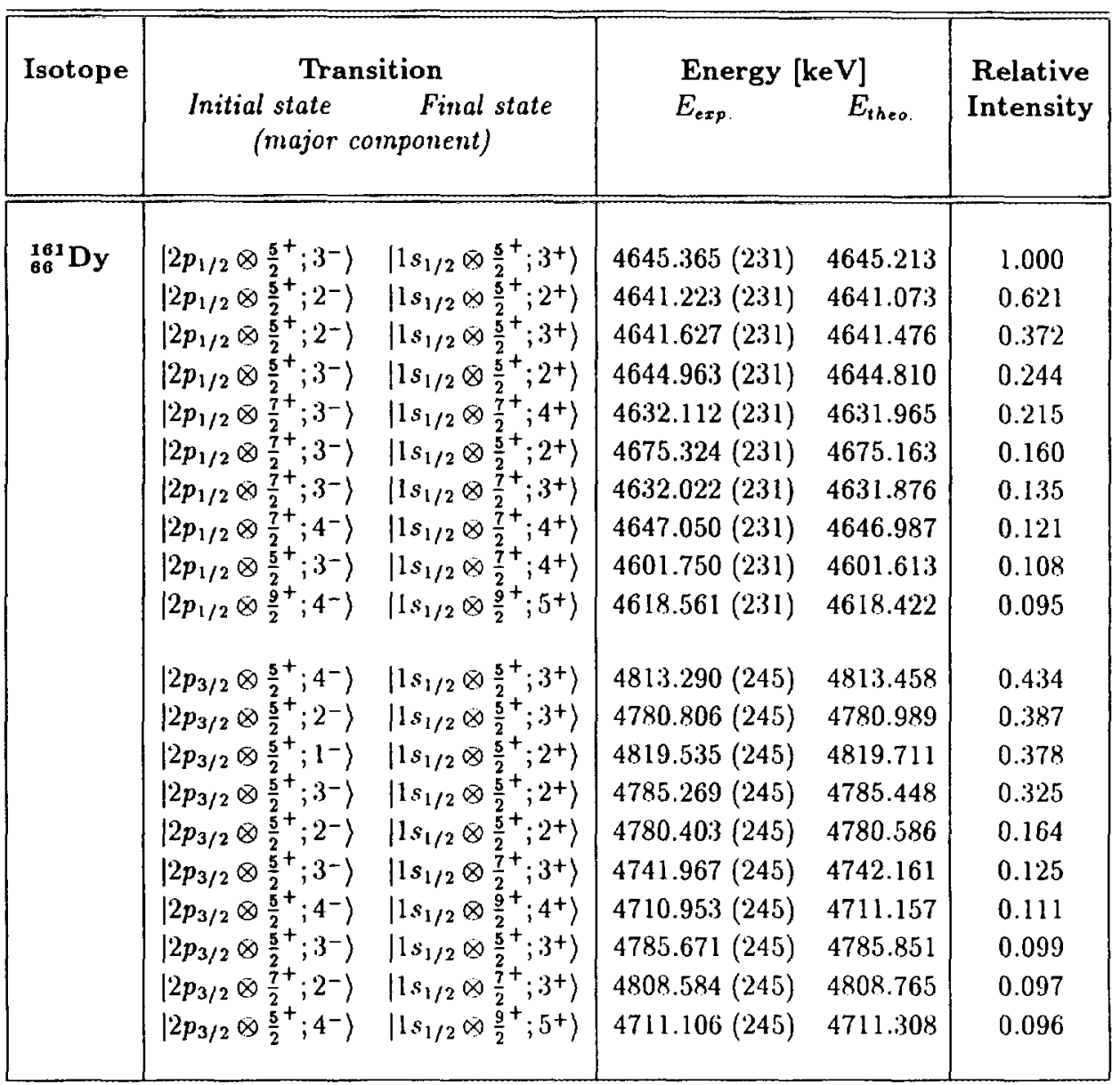

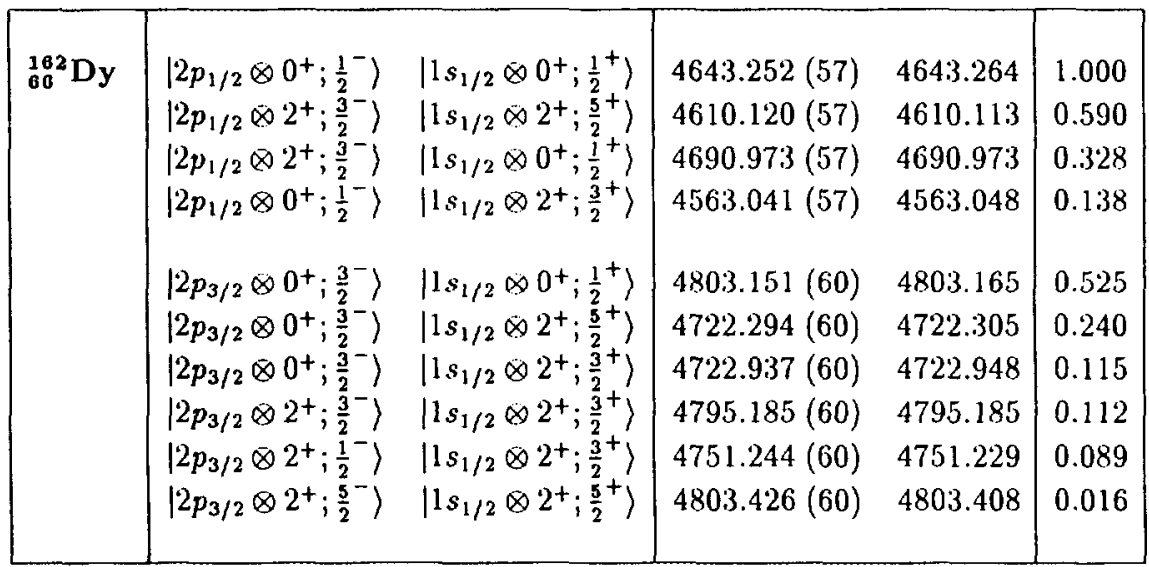


TABLE IIIB. Muonic $2 p \rightarrow 1 s$ Transition Energies and Relative Intensities of Deformed Nuclei with $60 \leqslant Z \leqslant 77$

See page 194 for Explanation of Tables

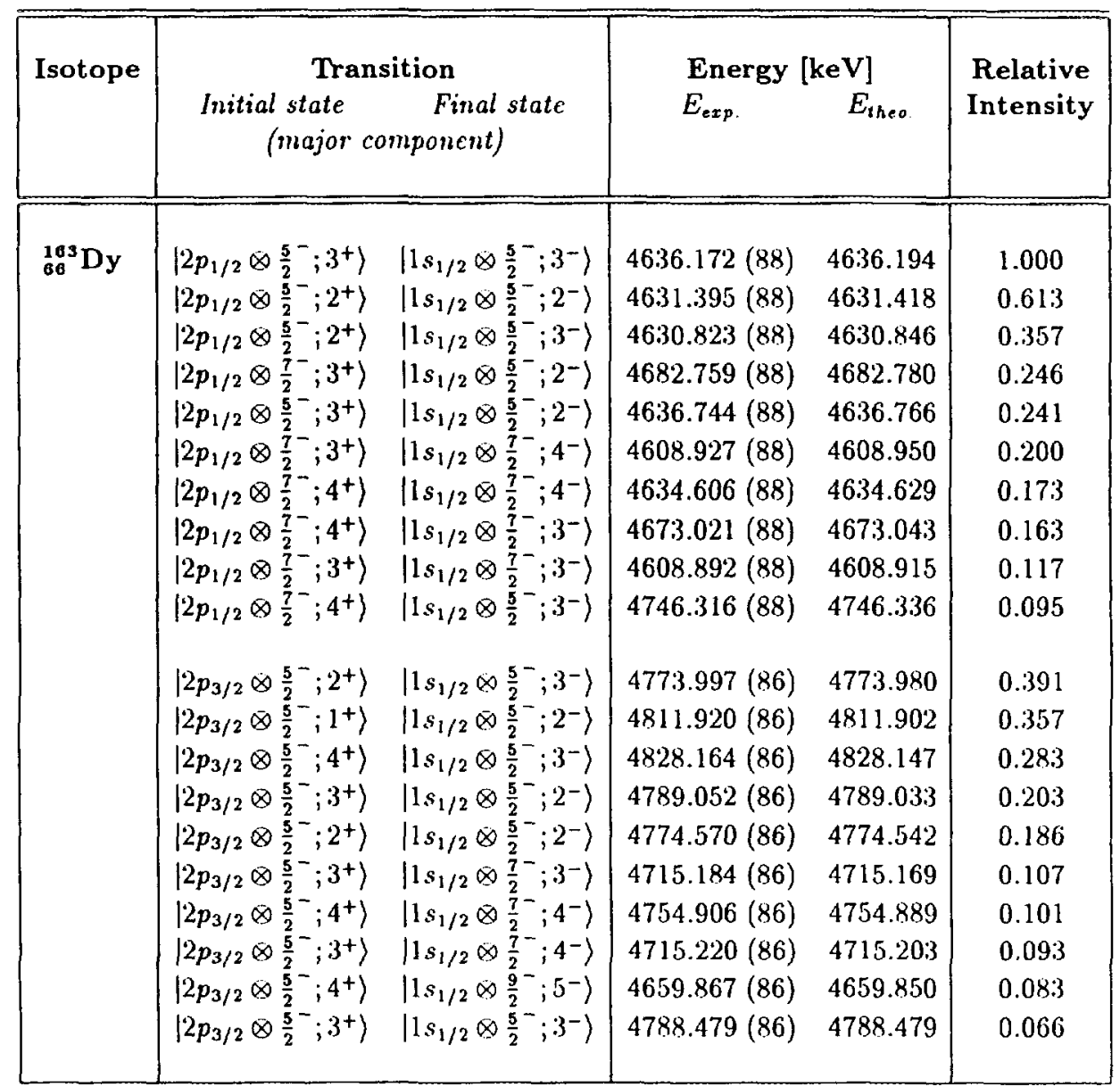

\begin{tabular}{|c|c|c|c|c|c|}
\hline${ }_{68}^{184} \mathrm{Dy}$ & $\begin{array}{l}\left|2 p_{1 / 2} \otimes 0^{+} ; \frac{1}{2}^{-}\right\rangle \\
\left|2 p_{1 / 2} \otimes 2^{+} ; \frac{3}{2}\right\rangle \\
\left|2 p_{1 / 2} \otimes 2^{+} ; \frac{3}{2}\right\rangle \\
\left|2 p_{1 / 2} \otimes 0^{+} ; \frac{1}{2}^{-}\right\rangle \\
\left|2 p_{3 / 2} \otimes 0^{+} ; \frac{3}{2}-\right\rangle \\
\left|2 p_{3 / 2} \otimes 0^{+} ; \frac{3}{2}-\right\rangle \\
\left|2 p_{3 / 2} \otimes 0^{+} ; \frac{3}{2}^{-}\right\rangle \\
\left|2 p_{3 / 2} \otimes 2^{+} ; \frac{3}{2}^{-}\right\rangle \\
\left|2 p_{3 / 2} \otimes 2^{+} ; \frac{1}{2}\right\rangle \\
\left|2 p_{3 / 2} \otimes 2^{+} ; \frac{5}{2}^{-}\right\rangle\end{array}$ & $\begin{array}{l}\left|1 s_{1 / 2} \otimes 0^{+} ; \frac{1}{2}^{+}\right\rangle \\
\left|1 s_{1 / 2} \otimes 2^{+} ; \frac{5}{2}^{+}\right\rangle \\
\left|1 s_{1 / 2} \otimes 0^{+} ; \frac{1}{2}^{+}\right\rangle \\
\left|1 s_{1 / 2} \otimes 2^{+} ; \frac{3}{2}^{+}\right\rangle \\
\left|1 s_{1 / 2} \otimes 0^{+} ; \frac{1}{2}^{+}\right\rangle \\
\left|1 s_{1 / 2} \otimes 2^{+} ; \frac{5}{2}^{+}\right\rangle \\
\left|1 s_{1 / 2} \otimes 2^{+} ; \frac{3}{2}^{+}\right\rangle \\
\left|1 s_{1 / 2} \otimes 2^{+} ; \frac{3}{2}^{+}\right\rangle \\
\left|1 s_{1 / 2} \otimes 2^{+} ; \frac{3}{2}^{+}\right\rangle \\
\left|1 s_{1 / 2} \otimes 2^{+} ; \frac{5}{2}^{+}\right\rangle\end{array}$ & $\begin{array}{l}4632.431(180) \\
4601.669(180) \\
4675.456(180) \\
4559.233(180) \\
4791.566(160) \\
4717.779(160) \\
4718.368(160) \\
4786.975(160) \\
4742.312(160) \\
4794.664(160)\end{array}$ & $\begin{array}{l}4632.372 \\
4601.616 \\
4675.378 \\
4559.199 \\
\\
4791.592 \\
4717.830 \\
4718.419 \\
4786.997 \\
4742.352 \\
4794.684\end{array}$ & $\begin{array}{l}1.000 \\
0.612 \\
0.302 \\
0.155 \\
\\
\\
0.560 \\
0.229 \\
0.133 \\
0.128 \\
0.097 \\
0.022\end{array}$ \\
\hline
\end{tabular}


TABLE IIIB. Muonic $2 p \rightarrow 1 s$ Transition Energies and Relative Intensities of Deformed Nuclei with $60 \leqslant Z \leqslant 77$

See page 194 for Explanation of Tables

\begin{tabular}{|c|c|c|c|c|}
\hline \multirow[t]{2}{*}{ Isotope } & Transition & \multicolumn{2}{|c|}{ Energy $[\mathrm{keV}]$} & \multirow{2}{*}{$\begin{array}{l}\text { Relative } \\
\text { Intensity }\end{array}$} \\
\hline & $\begin{array}{c}\text { Initial state } \quad \text { Final state } \\
\text { (major component) }\end{array}$ & $E_{\text {exp }}$ & $E_{\text {theo }}$ & \\
\hline \multirow[t]{10}{*}{${ }_{68}^{168} \mathrm{Er}$} & $\left|2 p_{1 / 2} \otimes 0^{+} ; \frac{1}{2}^{-}\right\rangle \quad\left|1 s_{1 / 2} \otimes 0^{+} ; \frac{1}{2}^{+}\right\rangle$ & $4790.858(123)$ & 4790.869 & 1.000 \\
\hline & $\left|2 p_{1 / 2} \otimes 2^{+} ; \frac{3}{2}^{-}\right\rangle \quad\left|1 s_{1 / 2} \otimes 2^{+} ; \frac{5}{2}^{+}\right\rangle$ & $4757.059(123)$ & 4757.072 & 0.607 \\
\hline & $\left|2 p_{1 / 2} \otimes 2^{+} ; \frac{3}{2}^{-}\right\rangle \quad\left|1 s_{1 / 2} \otimes 0^{+} ; \frac{1}{2}^{+}\right\rangle$ & $4838.748(123)$ & 4838.757 & 0.307 \\
\hline & $\left|2 p_{1 / 2} \otimes 0^{+} ; \frac{1}{2}^{-}\right\rangle \quad\left|1 s_{1 / 2} \otimes 2^{+} ; \frac{3}{2}^{+}\right\rangle$ & $4709.645(123)$ & 4709.658 & 0.151 \\
\hline & $\left|2 p_{3 / 2} \otimes 0^{+} ; \frac{3}{2}^{-}\right\rangle \quad\left|1 s_{1 / 2} \otimes 0^{+} ; \frac{1}{2}^{+}\right\rangle$ & $4963.709(157)$ & 4963.689 & $0.5: 37$ \\
\hline & $\left|2 p_{3 / 2} \otimes 0^{+} ; \frac{3}{2}^{-}\right\rangle \quad\left|1 s_{1 / 2} \otimes 2^{+} ; \frac{5}{2}^{+}\right\rangle$ & $4882.020(157)$ & 4882.003 & 0.221 \\
\hline & $\left|2 p_{3 / 2} \otimes 0^{+} ; \frac{3}{2}^{-}\right\rangle$ & $4882.496(157)$ & 4882.478 & 0.133 \\
\hline & $\left|2 p_{3 / 2} \otimes 2^{+} ; \frac{3}{2}^{-}\right\rangle \quad\left|1 s_{1 / 2} \otimes 2^{+} ; \frac{3}{2}^{+}\right\rangle$ & $4957.499(157)$ & 4957.479 & 0.125 \\
\hline & $\left|2 p_{3 / 2} \otimes 2^{+} ; \frac{1}{2}^{-}\right\rangle \quad\left|1 s_{1 / 2} \otimes 2^{+} ; \frac{3}{2}^{+}\right\rangle$ & $4909.981(157)$ & 4909.964 & 0.096 \\
\hline & $\left|2 p_{3 / 2} \otimes 2^{+} ; \frac{5}{2}^{-}\right\rangle \quad\left|1 s_{1 / 2} \otimes 2^{+} ; \frac{5}{2}^{+}\right\rangle$ & $4965.379(157)$ & 4965.365 & 0.022 \\
\hline
\end{tabular}

\begin{tabular}{|c|c|c|c|c|c|}
\hline${ }_{68}^{167} \mathrm{Er}$ & $\begin{array}{l}\left|2 p_{1 / 2} \otimes \frac{7}{2}^{+} ; 4^{-}\right\rangle \\
\left|2 p_{1 / 2} \otimes \frac{7}{2}^{+} ; 3^{-}\right\rangle \\
\left|2 p_{1 / 2} \otimes \frac{7}{2}^{+} ; 3^{-}\right\rangle \\
\left|2 p_{1 / 2} \otimes \frac{7}{2}^{+} ; 4^{-}\right\rangle \\
\left|2 p_{1 / 2} \otimes \frac{9}{2}^{+} ; 4^{-}\right\rangle \\
\left|2 p_{1 / 2} \otimes \frac{9}{2}^{+} ; 4^{-}\right\rangle \\
\left|2 p_{1 / 2} \otimes \frac{9}{2}^{+} ; 5^{-}\right\rangle \\
\left|2 p_{1 / 2} \otimes \frac{9}{2}^{+} ; 4^{-}\right\rangle \\
\left|2 p_{1 / 2} \otimes \frac{11}{2}^{+} ; 5^{-}\right\rangle \\
\left|2 p_{1 / 2} \otimes \frac{7}{2}^{+} ; 4^{-}\right\rangle \\
\left|2 p_{3 / 2} \otimes \frac{7}{2}^{+} ; 3^{-}\right\rangle \\
\left|2 p_{3 / 2} \otimes \frac{7}{2}^{+} ; 2^{-}\right\rangle \\
\left|2 p_{3 / 2} \otimes \frac{7}{2}^{+} ; 5^{-}\right\rangle \\
\left|2 p_{3 / 2} \otimes \frac{7}{2}^{+} ; 4^{-}\right\rangle \\
\left|2 p_{3 / 2} \otimes \frac{7}{2}^{+} ; 3^{-}\right\rangle \\
\left|2 p_{3 / 2} \otimes \frac{7}{2}^{+} ; 4^{-}\right\rangle \\
\left|2 p_{3 / 2} \otimes \frac{7}{2}^{+} ; 5^{-}\right\rangle \\
\left|2 p_{3 / 2} \otimes \frac{7}{2}^{+} ; 4^{-}\right\rangle \\
\left|2 p_{3 / 2} \otimes \frac{7}{2}^{+} ; 5^{+}\right\rangle \\
\left|2 p_{3 / 2} \otimes \frac{7}{2}^{+} ; 4^{-}\right\rangle\end{array}$ & $\begin{array}{l}\left|1 s_{1 / 2} \otimes \frac{7}{2}^{+} ; 4^{+}\right\rangle \\
\left|1 s_{1 / 2} \otimes \frac{7}{2} ; 3^{+}\right\rangle \\
\left|1 s_{1 / 2} \otimes \frac{7}{2} ; 4^{+}\right\rangle \\
\left|1 s_{1 / 2} \otimes \frac{7}{2} ; 3^{+}\right\rangle \\
\left|1 s_{1 / 2} \otimes \frac{7}{2} ; 3^{+}\right\rangle \\
\left|1 s_{1 / 2} \otimes \frac{9}{2} ; 5^{+}\right\rangle \\
\left|1 s_{1 / 2} \otimes \frac{7}{2} ; 4^{+}\right\rangle \\
\left|1 s_{1 / 2} \otimes \frac{9}{2} ; 4^{+}\right\rangle \\
\left|1 s_{1 / 2} \otimes \frac{9}{2} ; 4^{+}\right\rangle \\
\left|1 s_{1 / 2} \otimes \frac{9}{2}^{+} ; 4^{+}\right\rangle \\
\left|1 s_{1 / 2} \otimes \frac{7}{2} ; 4^{+}\right\rangle \\
\left|1 s_{1 / 2} \otimes \frac{7}{2}^{+} ; 3^{+}\right\rangle \\
\left|1 s_{1 / 2} \otimes \frac{7}{2}^{+} ; 4^{+}\right\rangle \\
\left|1 s_{1 / 2} \otimes \frac{7}{2}^{+} ; 3^{+}\right\rangle \\
\left|1 s_{1 / 2} \otimes \frac{7}{2} ; 3^{+}\right\rangle \\
\left|1 s_{1 / 2} \otimes \frac{9}{2} ; 5^{+}\right\rangle \\
\left|1 s_{1 / 2} \otimes \frac{9}{2}^{+} ; 5^{+}\right\rangle \\
\left|1 s_{1 / 2} \otimes \frac{7}{2}^{+} ; 5^{+}\right\rangle \\
\left|1 s_{1 / 2} \otimes \frac{11}{2}^{+} ; 6^{+}\right\rangle \\
\left|1 s_{1 / 2} \otimes \frac{7}{2}^{+} ; 4^{+}\right\rangle\end{array}$ & $\begin{array}{l}4781.145(120) \\
4775.911(120) \\
4776.377(120) \\
4780.680(120) \\
4837.005(120) \\
4757.831(120) \\
4781.279(120) \\
4757.814(120) \\
4829.394(120) \\
4701.508(120) \\
4931.087(99) \\
4974.696(99) \\
4987.093(99) \\
4944.811(99) \\
4930.624(99) \\
4865.619(99) \\
4907.456(99) \\
4865.638(99) \\
4807.786(99) \\
4945.274(99)\end{array}$ & $\begin{array}{l}4781.031 \\
4775.798 \\
4776.262 \\
4780.567 \\
4836.887 \\
4757.716 \\
4781.164 \\
4757.698 \\
4829.275 \\
4701.396 \\
4931.115 \\
4974.720 \\
4987.120 \\
4944.840 \\
4930.651 \\
4865.651 \\
4907.485 \\
4865.669 \\
4807.815 \\
4945.304\end{array}$ & $\begin{array}{l}1.000 \\
0.710 \\
0.305 \\
0.205 \\
0.195 \\
0.145 \\
0.135 \\
0.135 \\
0.120 \\
0.070 \\
\\
0.428 \\
0.414 \\
0.312 \\
0.215 \\
0.161 \\
0.101 \\
0.076 \\
0.075 \\
0.075 \\
0.062\end{array}$ \\
\hline
\end{tabular}


G. FRICKE et al. Nuclear Charge Radii

TABLE IIIB. Muonic $2 p \rightarrow 1 s$ Transition Energies and Relative Intensities of Deformed Nuclei with $60 \leqslant Z \leqslant 77$

See page 194 for Explanation of Tables

\begin{tabular}{|c|c|c|c|c|}
\hline Isotope & $\begin{array}{c}\text { Transition } \\
\text { Initial state Final state } \\
\text { (major component) }\end{array}$ & $\begin{array}{l}\text { Energy [k } \\
E_{\text {exp. }}\end{array}$ & $\frac{\mathrm{eV}]}{E_{\text {theo. }}}$ & $\begin{array}{l}\text { Relative } \\
\text { Intensity }\end{array}$ \\
\hline${ }_{68}^{188} \mathrm{Er}$ & $\begin{array}{ll}\left|2 p_{1 / 2} \otimes 0^{+} ; \frac{1}{2}^{-}\right\rangle & \left|1 s_{1 / 2} \otimes 0^{+} ; \frac{1}{2}^{+}\right\rangle \\
\left|2 p_{1 / 2} \otimes 2^{+} ; \frac{3}{2}^{-}\right\rangle & \left|1 s_{1 / 2} \otimes 2^{+} ; \frac{5}{2}^{+}\right\rangle \\
\left|2 p_{1 / 2} \otimes 2^{+} ; \frac{3}{2}^{-}\right\rangle & \left|1 s_{1 / 2} \otimes 0^{+} ; \frac{1}{2}^{+}\right\rangle \\
\left|2 p_{1 / 2} \otimes 0^{+} ; \frac{1}{2}^{-}\right\rangle & \left|1 s_{1 / 2} \otimes 2^{+} ; \frac{3}{2}^{+}\right\rangle \\
\left|2 p_{3 / 2} \otimes 0^{+} ; \frac{3}{2}^{-}\right\rangle & \left|1 s_{1 / 2} \otimes 0^{+} ; \frac{1}{2}^{+}\right\rangle \\
\left|2 p_{3 / 2} \otimes 0^{+} ; \frac{3}{2}^{-}\right\rangle & \left|1 s_{1 / 2} \otimes 2^{+} ; \frac{5}{2}^{+}\right\rangle \\
\left|2 p_{3 / 2} \otimes 0^{+} ; \frac{3}{2}-\right\rangle & \left|1 s_{1 / 2} \otimes 2^{+} ; \frac{3}{2}^{+}\right\rangle \\
\left|2 p_{3 / 2} \otimes 2^{+} ; \frac{3}{2}^{-}\right\rangle & \left|1 s_{1 / 2} \otimes 2^{+} ; \frac{3}{2}^{+}\right\rangle \\
\left|2 p_{3 / 2} \otimes 2^{+} ; \frac{1}{2}^{-}\right\rangle & \left|1 s_{1 / 2} \otimes 2^{+} ; \frac{3}{2}^{+}\right\rangle \\
\left|2 p_{3 / 2} \otimes 2^{+} ; \frac{5}{2}^{-}\right\rangle & \left|1 s_{1 / 2} \otimes 2^{+} ; \frac{5}{2}^{+}\right\rangle\end{array}$ & $\begin{array}{l}4780.221(89) \\
4746.844(89) \\
4827.057(89) \\
4700.586(89) \\
\\
4954.628(101) \\
4874.415(101) \\
4874.993(101) \\
4949.263(101) \\
4901.723(101) \\
4956.622(101)\end{array}$ & $\begin{array}{l}4780.270 \\
4746.843 \\
4827.052 \\
4700.637 \\
4954.656 \\
4874.447 \\
4875.023 \\
4949.231 \\
4901.790 \\
4956.565\end{array}$ & $\begin{array}{l}1.000 \\
0.621 \\
0.325 \\
0.153 \\
0.138 \\
0.057 \\
0.035 \\
0.033 \\
0.024 \\
0.006\end{array}$ \\
\hline
\end{tabular}

\begin{tabular}{|c|c|c|c|c|c|}
\hline${ }_{68}^{170} \mathrm{Er}$ & $\begin{array}{l}\left|2 p_{1 / 2} \otimes 0^{+} ; \frac{1}{2}^{-}\right\rangle \\
\left|2 p_{1 / 2} \otimes 2^{+} ; \frac{3}{2}^{-}\right\rangle \\
\left|2 p_{1 / 2} \otimes 2^{+} ; \frac{3}{2}^{-}\right\rangle \\
\left|2 p_{1 / 2} \otimes 0^{+} ; \frac{1}{2}^{-}\right\rangle \\
\left|2 p_{3 / 2} \otimes 0^{+} ; \frac{3}{2}-\right\rangle \\
\left|2 p_{3 / 2} \otimes 0^{+} ; \frac{3}{2}^{-}\right\rangle \\
\left|2 p_{3 / 2} \otimes 0^{+} ; \frac{3}{2}^{-}\right\rangle \\
\left|2 p_{3 / 2} \otimes 2^{+} ; \frac{3}{2}^{-}\right\rangle \\
\left|2 p_{3 / 2} \otimes 2^{+} ; \frac{1}{2}-\right\rangle \\
\left|2 p_{3 / 2} \otimes 2^{+} ; \frac{5}{2}^{-}\right\rangle\end{array}$ & $\begin{array}{l}\left|1 s_{1 / 2} \otimes 0^{+} ; \frac{1}{2}^{+}\right\rangle \\
\left|1 s_{1 / 2} \otimes 2^{+} ; \frac{5}{2}^{+}\right\rangle \\
\left.\mid 1 s_{1 / 2} \otimes 0^{+} ; \frac{1}{2}^{+}\right\} \\
\left|1 s_{1 / 2} \otimes 2^{+} ; \frac{3}{2}^{+}\right\rangle \\
\left.\mid 1 s_{1 / 2} \otimes 0^{+} ; \frac{1}{2}^{+}\right\} \\
\left|1 s_{1 / 2} \otimes 2^{+} ; \frac{5}{2}^{+}\right\rangle \\
\left|1 s_{1 / 2} \otimes 2^{+} ; \frac{3}{2}^{+}\right\rangle \\
\left|1 s_{1 / 2} \otimes 2^{+} ; \frac{3}{2}^{+}\right\rangle \\
\left.\mid 1 s_{1 / 2} \otimes 2^{+} ; \frac{3}{2}^{+}\right\} \\
\left|1 s_{1 / 2} \otimes 2^{+} ; \frac{5}{2}^{+}\right\rangle\end{array}$ & $\begin{array}{l}4770.262(98) \\
4737.271(98) \\
4816.399(98) \\
4691.756(98) \\
4943.467(108) \\
4864.337(108) \\
4864.960(108) \\
4938.771(108) \\
4890.855(108) \\
4945.814(108)\end{array}$ & $\begin{array}{l}4770.201 \\
4737.182 \\
4816.308 \\
4691.699 \\
4943.552 \\
4864.426 \\
4865.051 \\
4938.830 \\
4890.934 \\
4945.805\end{array}$ & $\begin{array}{l}0.493 \\
0.124 \\
0.116 \\
0.084 \\
0.021 \\
0.015\end{array}$ \\
\hline
\end{tabular}


TABLE IIIB. Muonic $2 p \rightarrow 1 s$ Transition Energies and Relative Intensities of Deformed Nuclei with $60 \leqslant Z \leqslant 77$

See page 194 for Explanation of Tables

\begin{tabular}{|c|c|c|c|c|}
\hline \multirow[t]{2}{*}{ Isotope } & Transition & \multicolumn{2}{|c|}{ Energy [keV] } & \multirow{2}{*}{$\begin{array}{l}\text { Relative } \\
\text { Intensity }\end{array}$} \\
\hline & $\begin{array}{c}\text { Initial state Final state } \\
\text { (major component) }\end{array}$ & $E_{\text {exp }}$ & $E_{\text {theo }}$ & \\
\hline \multirow[t]{10}{*}{${ }_{70}^{174} \mathrm{Yb}$} & $\left|2 p_{1 / 2} \otimes 0^{+} ; \frac{1}{2}^{-}\right\rangle \quad\left|1 s_{1 / 2} \otimes 0^{+} ; \frac{1}{2}^{+}\right\rangle$ & $4918.175(74)$ & 4918.169 & 1.000 \\
\hline & $\left|2 p_{1 / 2} \otimes 2^{+} ; \frac{3}{2}^{-}\right\rangle \quad\left|1 s_{1 / 2} \otimes 2^{+} ; \frac{5}{2}^{+}\right\rangle$ & $4887.344(74)$ & 4887.349 & 0.621 \\
\hline & $\left|2 p_{1 / 2} \otimes 2^{+} ; \frac{3}{2}^{-}\right\rangle \quad\left|1 s_{1 / 2} \otimes 0^{+} ; \frac{1}{2}^{+}\right\rangle$ & $4963.997(74)$ & 4963.971 & 0.271 \\
\hline & $\left|2 p_{1 / 2} \otimes 0^{+} ; \frac{1}{2}^{-}\right\rangle \quad\left|1 s_{1 / 2} \otimes 2^{+} ; \frac{3}{2}^{+}\right\rangle$ & $4841.498(74)$ & 4841.529 & 0.166 \\
\hline & $\left|2 p_{3 / 2} \otimes 0^{+} ; \frac{3}{2}^{-}\right\rangle \quad\left|1 s_{1 / 2} \otimes 0^{+} ; \frac{1}{2}^{+}\right\rangle$ & $5100.914(79)$ & 5100.898 & 0.575 \\
\hline & $\left|2 p_{3 / 2} \otimes 0^{+} ; \frac{3}{2}^{-}\right\rangle \quad\left|1 s_{1 / 2} \otimes 2^{+} ; \frac{5}{2}^{+}\right\rangle$ & $5024.261(79)$ & 5024.276 & 0.201 \\
\hline & $\left|2 p_{3 / 2} \otimes 0^{+} ; \frac{3}{2}^{-}\right\rangle \quad\left|1 s_{1 / 2} \otimes 2^{+} ; \frac{3}{2}^{+}\right\rangle$ & $5024.238(79)$ & 5024.258 & 0.159 \\
\hline & $\left|2 p_{3 / 2} \otimes 2^{+} ; \frac{3}{2}^{-}\right\rangle \quad\left|1 s_{1 / 2} \otimes 2^{+} ; \frac{3}{2}^{+}\right\rangle$ & $5100.184(79)$ & 5100.205 & 0.139 \\
\hline & $\left|2 p_{3 / 2} \otimes 2^{+} ; \frac{1}{2}^{-}\right\rangle \quad\left|1 s_{1 / 2} \otimes 2^{+} ; \frac{3}{2}^{+}\right|$ & $5049.073(79)$ & 5049.091 & 0.092 \\
\hline & $\left|2 p_{3 / 2} \otimes 2^{+} ; \frac{5}{2}^{-}\right\rangle \quad \mid 1 s_{1 / 2} \otimes 2^{+} ; \frac{5}{2}^{+}$ & $5106.883(79)$ & 5106.881 & 0.030 \\
\hline
\end{tabular}

\begin{tabular}{|c|c|c|c|c|c|}
\hline${ }_{72}^{180} \mathrm{Hf}$ & $\begin{array}{l}\left|2 p_{1 / 2} \otimes 0^{+} ; \frac{1}{2}^{-}\right\rangle \\
\left|2 p_{1 / 2} \otimes 2^{+} ; \frac{3}{}^{-}\right\rangle \\
\left|2 p_{1 / 2} \otimes 2^{+} ; \frac{3}{2}-\right\rangle \\
\left|2 p_{1 / 2} \otimes 0^{+} ; \frac{1}{2}^{-}\right\rangle \\
\left|2 p_{3 / 2} \otimes 0^{+} ; \frac{3}{2}^{-}\right\rangle \\
\left|2 p_{3 / 2} \otimes 0^{+} ; \frac{3}{2}^{-}\right\rangle \\
\left|2 p_{3 / 2} \otimes 0^{+} ; \frac{3}{2}^{-}\right\rangle \\
\left|2 p_{3 / 2} \otimes 2^{+} ; \frac{3}{2}^{-}\right\rangle \\
\left|2 p_{3 / 2} \otimes 2^{+} ; \frac{1^{-}}{}\right\rangle \\
\left|2 p_{3 / 2} \otimes 2^{+} ; \frac{5}{2}^{-}\right\rangle\end{array}$ & $\begin{array}{l}\left|1 s_{1 / 2} \otimes 0^{+} ; \frac{1}{2}^{+}\right\rangle \\
\left|1 s_{1 / 2} \otimes 2^{+} ; \frac{5}{2}^{+}\right\rangle \\
\mid 1 s_{1 / 2} \otimes 0^{+} ; \frac{1}{2}+ \\
\left|1 s_{1 / 2} \otimes 2^{+} ; \frac{3}{2}^{+}\right\rangle \\
\left|1 s_{1 / 2} \otimes 0^{+} ; \frac{1}{2}^{+}\right\rangle \\
\mid 1 s_{1 / 2} \otimes 2^{+} ; \frac{5}{2}+ \\
\left|1 s_{1 / 2} \otimes 2^{+} ; \frac{3}{2}^{+}\right\rangle \\
\left|1 s_{1 / 2} \otimes 2^{+} ; \frac{3}{2}^{+}\right\rangle \\
\left|1 s_{1 / 2} \otimes 2^{+} ; \frac{3}{2}^{+}\right\rangle \\
\left|1 s_{1 / 2} \otimes 2^{+} ; \frac{5}{2}^{+}\right\rangle\end{array}$ & $\begin{array}{l}5068.614(103) \\
5036.020(103) \\
5128.832(103) \\
4976.243(103) \\
5254.559(114) \\
5161.741(114) \\
5162.190(114) \\
5250.319(114) \\
5199.335(114) \\
5259.301(114)\end{array}$ & $\begin{array}{l}5068.619 \\
5036.020 \\
5128.836 \\
4976.254 \\
\\
5254.553 \\
5161.737 \\
5162.187 \\
5250.313 \\
5199.3330 \\
5259.294\end{array}$ & $\begin{array}{l}1.000 \\
0.536 \\
0.253 \\
0.115 \\
\\
0.582 \\
0.208 \\
0.124 \\
0.095 \\
0.064 \\
0.017\end{array}$ \\
\hline
\end{tabular}


TABLE IIIB. Muonic $2 p \rightarrow 1 s$ Transition Energies and Relative Intensities of Deformed Nuclei with $60 \leqslant Z \leqslant 77$

See page 194 for Explanation of Tables

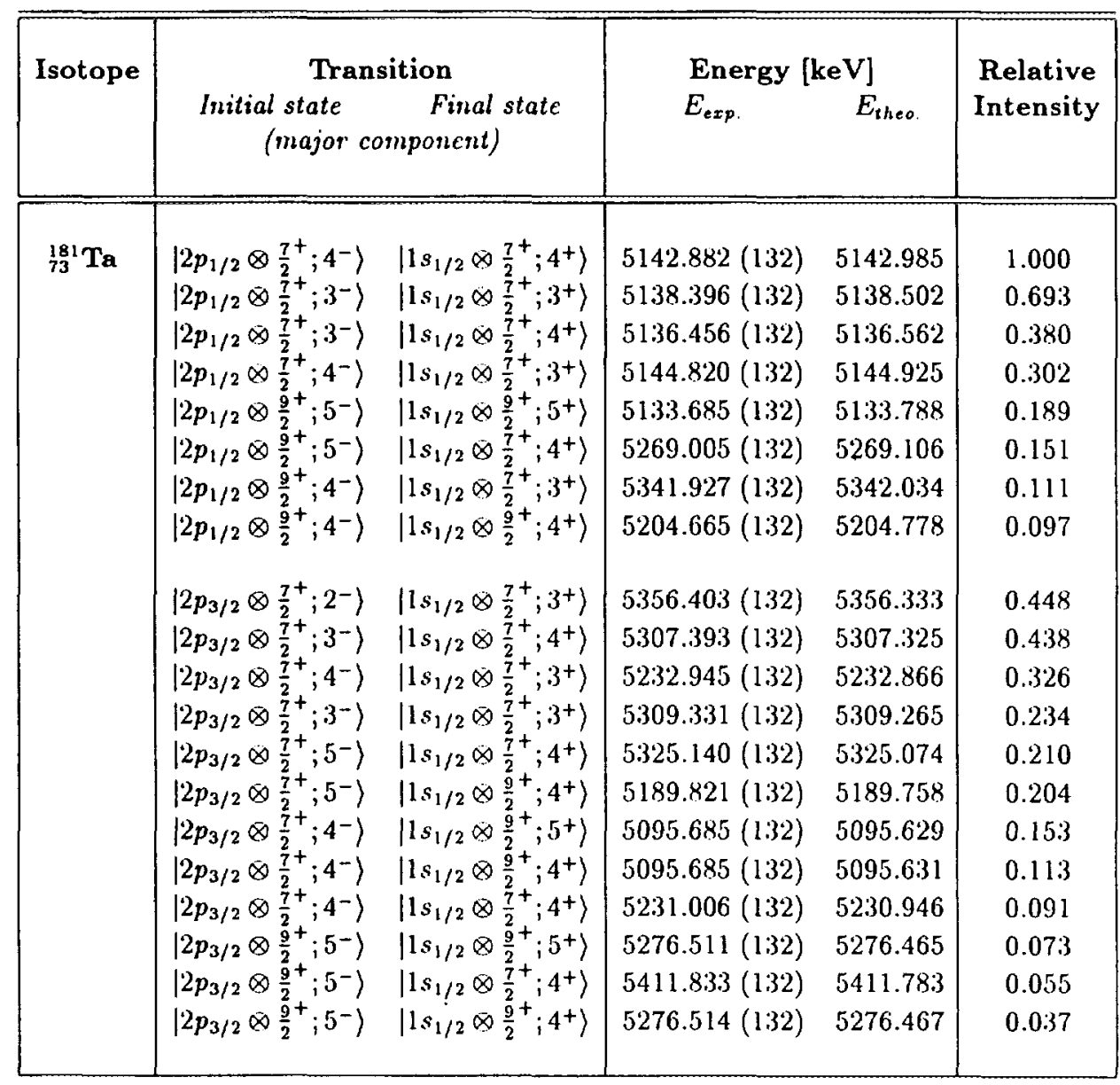


TABLE IIIB. Muonic $2 p \rightarrow 1 s$ Transition Energies and Relative Intensities of Deformed Nuclei with $60 \leqslant Z \leqslant 77$

See page 194 for Explanation of Tables

\begin{tabular}{|c|c|c|c|c|}
\hline \multirow[t]{2}{*}{ Isotope } & Transition & \multicolumn{2}{|c|}{ Energy [keV] } & \multirow{2}{*}{$\begin{array}{l}\text { Relative } \\
\text { Intensity }\end{array}$} \\
\hline & $\begin{array}{c}\text { Initial state Final state } \\
\text { (major component) }\end{array}$ & $E_{\text {exp }}$ & $E_{\text {theo }}$ & \\
\hline \multirow[t]{10}{*}{${ }_{74}^{182} W$} & $\left|2 p_{1 / 2} \otimes 0^{+} ; \frac{1}{2}^{-}\right\rangle \quad\left|1 s_{1 / 2} \otimes 0^{+} ; \frac{1}{2}^{+}\right\rangle$ & $5227.401(156)$ & 5227.421 & 1.000 \\
\hline & $\left|2 p_{1 / 2} \otimes 2^{+} ; \frac{3}{2}^{-}\right\rangle \quad\left|1 s_{1 / 2} \otimes 2^{+} ; \frac{5}{2}^{+}\right\rangle$ & $5196.613(156)$ & 5196.646 & 0.486 \\
\hline & $\left|2 p_{1 / 2} \otimes 2^{+} ; \frac{3}{2}^{-}\right\rangle \quad\left|1 s_{1 / 2} \otimes 0^{+} ; \frac{1}{2}^{+}\right\rangle$ & $5296.680(156)$ & 5296.709 & 0.227 \\
\hline & $\left|2 p_{1 / 2} \otimes 0^{+} ; \frac{1}{2}^{-}\right\rangle \quad\left|1 s_{1 / 2} \otimes 2^{+} ; \frac{3}{2}^{+}\right\rangle$ & $5127.350(156)$ & 5127.371 & 0.092 \\
\hline & $\left|2 p_{3 / 2} \otimes 0^{+} ; \frac{3}{2}^{-}\right\rangle \quad\left|1 s_{1 / 2} \otimes 0^{+} ; \frac{1}{2}^{+}\right\rangle$ & $5419.888(178)$ & 5419.864 & 0.580 \\
\hline & $\left.\left|2 p_{3 / 2} \otimes 0^{+} ; \frac{3}{2}^{-}\right\rangle \quad \mid 1 s_{1 / 2} \otimes 2^{+} ; \frac{5}{2}^{+}\right\}$ & $5319.823(178)$ & $5: 319.801$ & 0.192 \\
\hline & $\left|2 p_{3 / 2} \otimes 0^{+} ; \frac{3}{2}^{-}\right\rangle \quad\left|1 s_{1 / 2} \otimes 2^{+} ; \frac{3}{2}^{+}\right\rangle$ & $5319.834(178)$ & 5319.814 & 0.132 \\
\hline & $\left|2 p_{3 / 2} \otimes 2^{+} ; \frac{3}{2}^{-}\right\rangle \quad\left|1 s_{1 / 2} \otimes 2^{+} ; \frac{3}{2}^{+}\right\rangle$ & $5414.544(178)$ & 5414.520 & 0.090 \\
\hline & $\left|2 p_{3 / 2} \otimes 2^{+} ; \frac{1}{2}^{-}\right\rangle \quad\left|1 s_{1 / 2} \otimes 2^{+} ; \frac{3}{2}^{+}\right\rangle$ & $5367.270(178)$ & 5367.266 & 0.052 \\
\hline & $\left|2 p_{3 / 2} \otimes 2^{+} ; \frac{5}{2}^{-}\right\rangle \quad\left|1 s_{1 / 2} \otimes 2^{+} ; \frac{5}{2}^{+}\right\rangle$ & $5423.889(178)$ & 5423.864 & 0.013 \\
\hline
\end{tabular}

\begin{tabular}{|c|c|c|c|c|c|}
\hline${ }_{74}^{184} \mathrm{~W}$ & $\begin{array}{l}\left|2 p_{1 / 2} \otimes 0^{+} ; \frac{1}{2}^{-}\right\rangle \\
\left|2 p_{1 / 2} \otimes 2^{+} ; \frac{3}{2}\right\rangle \\
\left|2 p_{1 / 2} \otimes 2^{+} ; \frac{3}{2}^{-}\right\rangle \\
\left|2 p_{1 / 2} \otimes 0^{+} ; \frac{1}{2}^{-}\right\rangle \\
\left|2 p_{3 / 2} \otimes 0^{+} ; \frac{3}{2}-\right\rangle \\
\left|2 p_{3 / 2} \otimes 0^{+} ; \frac{3}{2}-\right\rangle \\
\left|2 p_{3 / 2} \otimes 0^{+} ; \frac{3}{2}^{-}\right\rangle \\
\left|2 p_{3 / 2} \otimes 2^{+} ; \frac{3}{2}^{-}\right\rangle \\
\left|2 p_{3 / 2} \otimes 2^{+} ; \frac{1}{2}\right\rangle \\
\left|2 p_{3 / 2} \otimes 2^{+} ; \frac{5}{2}^{-}\right\rangle\end{array}$ & $\begin{array}{l}\left|1 s_{1 / 2} \otimes 0^{+} ; \frac{1}{2}^{+}\right\rangle \\
\left|1 s_{1 / 2} \otimes 2^{+} ; \frac{5}{2}^{+}\right\rangle \\
\left|1 s_{1 / 2} \otimes 0^{+} ; \frac{1}{2}^{+}\right\rangle \\
\left|1 s_{1 / 2} \otimes 2^{+} ; \frac{3}{2}^{+}\right\rangle \\
\left|1 s_{1 / 2} \otimes 0^{+} ; \frac{1}{2}^{+}\right\rangle \\
\left|1 s_{1 / 2} \otimes 2^{+} ; \frac{5}{2}^{+}\right\rangle \\
\mid 1 s_{1 / 2} \otimes 2^{+} ; \frac{3}{2}+ \\
\left|1 s_{1 / 2} \otimes 2^{+} ; \frac{3}{2}^{+}\right\rangle \\
\left|1 s_{1 / 2} \otimes 2^{+} ; \frac{3}{2}^{+}\right\rangle \\
\left|1 s_{1 / 2} \otimes 2^{+} ; \frac{5}{2}^{+}\right\rangle\end{array}$ & $\begin{array}{l}5222.414(137) \\
5187.721(137) \\
5300.924(137) \\
5109.550(137) \\
5413.612(158) \\
5300.412(158) \\
5300.746(158) \\
5401.973(158) \\
5357.019(158) \\
5414.810(158)\end{array}$ & $\begin{array}{l}5222.386 \\
5187.704 \\
5300.901 \\
5109.525 \\
5413.636 \\
5300.439 \\
5300.775 \\
5401.996 \\
5357.059 \\
5414.829\end{array}$ & $\begin{array}{l}1.000 \\
0.496 \\
0.276 \\
0.078 \\
\\
0.590 \\
0.231 \\
0.120 \\
0.077 \\
0.047 \\
0.008\end{array}$ \\
\hline
\end{tabular}

\begin{tabular}{|c|c|c|c|c|c|}
\hline${ }_{74}^{186} \mathrm{~W}$ & $\begin{array}{l}\left|2 p_{1 / 2} \otimes 0^{+} ; \frac{1}{2}^{-}\right\rangle \\
\left|2 p_{1 / 2} \otimes 2^{+} ; \frac{3}{2}^{-}\right\rangle \\
\left|2 p_{1 / 2} \otimes 2^{+} ; \frac{3}{2}^{-}\right\rangle \\
\left|2 p_{1 / 2} \otimes 0^{+} ; \frac{1}{2}^{-}\right\rangle \\
\left|2 p_{3 / 2} \otimes 0^{+} ; \frac{3}{2}^{-}\right\rangle \\
\left|2 p_{3 / 2} \otimes 0^{+} ; \frac{3}{2}^{-}\right\rangle \\
\left|2 p_{3 / 2} \otimes 0^{+} ; \frac{3}{2}^{-}\right\rangle \\
\left|2 p_{3 / 2} \otimes 2^{+} ; \frac{3}{2}^{-}\right\rangle \\
\left|2 p_{3 / 2} \otimes 2^{+} ; \frac{1}{2}^{-}\right\rangle \\
\left|2 p_{3 / 2} \otimes 2^{+} ; \frac{5}{2}^{-}\right\rangle\end{array}$ & $\begin{array}{l}\left|1 s_{1 / 2} \otimes 0^{+} ; \frac{1}{2}{ }^{+}\right\rangle \\
\left|1 s_{1 / 2} \otimes 2^{+} ; \frac{5}{2}\right\rangle \\
\mid 1 s_{1 / 2} \otimes 0^{+} ; \frac{1}{2}+ \\
\left|1 s_{1 / 2} \otimes 2^{+} ; \frac{3}{2}{ }^{+}\right\rangle \\
\left|1 s_{1 / 2} \otimes 0^{+} ; \frac{1}{2}+\right\rangle \\
\mid 1 s_{1 / 2} \otimes 2^{+} ; \frac{5}{2}+ \\
\left|1 s_{1 / 2} \otimes 2^{+} ; \frac{3}{2}^{+}\right\rangle \\
\mid 1 s_{1 / 2} \otimes 2^{+} ; \frac{3}{2}+ \\
\mid 1 s_{1 / 2} \otimes 2^{+} ; \frac{3}{2}+ \\
\left|1 s_{1 / 2} \otimes 2^{+} ; \frac{5}{2}^{+}\right\rangle\end{array}$ & $\begin{array}{l}5217.662(163) \\
5181.047(163) \\
5304.214(163) \\
5095.109(163) \\
\\
5408.225(188) \\
5285.059(188) \\
5285.676(188) \\
5393.672(188) \\
5349.116(188) \\
5405.740(188)\end{array}$ & $\begin{array}{l}5217.690 \\
5181.076 \\
5304.239 \\
5095.146 \\
\\
5408.201 \\
5285.038 \\
5285.657 \\
5393.642 \\
5349.096 \\
5405.709\end{array}$ & $\begin{array}{l}1.000 \\
0.493 \\
0.322 \\
0.064 \\
\\
0.595 \\
0.277 \\
0.106 \\
0.065 \\
0.042 \\
0.007\end{array}$ \\
\hline
\end{tabular}


TABLE IIIC. Barrett Radii and Related Parameters of Deformed Nuclei with $60 \leqslant Z \leqslant 77$ See page 194 for Explanation of Tables

\begin{tabular}{|c|c|c|c|c|c|c|c|c|c|c|}
\hline Isotope & $\begin{array}{l}\text { NPol } \\
\text { [keV] }\end{array}$ & $\begin{array}{c}\boldsymbol{R}_{\mathbf{0}} \\
{[\mathbf{f m}]}\end{array}$ & $\stackrel{a}{[\mathrm{fm}]}$ & $\beta_{2}$ & $\begin{array}{c}\left\langle r^{2}\right\rangle_{\text {model }}^{1 / 2} \\
{[\mathrm{fm}]}\end{array}$ & $\begin{array}{c}a \\
{[1 / f m]}\end{array}$ & $\mathbf{k}$ & $\begin{array}{c}C_{k} \\
{[\mathrm{am} / \mathrm{keV}]}\end{array}$ & $\begin{array}{l}R_{h \alpha}^{\mu} \\
{[\mathbf{f m}]}\end{array}$ & Ref. \\
\hline${ }^{150} N d^{\dagger}$ & $\begin{array}{r}-0.531 \\
-13.961\end{array}$ & 5.8821 & 0.523 & 0.278 & 5.047 & $\begin{array}{l}0.1381 \\
0.1359\end{array}$ & $\begin{array}{l}2.3456 \\
2.3369\end{array}$ & $\begin{array}{l}-2.387 \\
-2.331\end{array}$ & $\begin{array}{c}6.4422 \\
(1 ; 13) \\
6.4431 \\
(1 ; 18)\end{array}$ & [Be92] \\
\hline${ }^{144} \mathrm{Sm}$ & $\begin{array}{l}1.941 \\
2.003\end{array}$ & 5.8624 & 0.523 & 0.090 & 4.949 & $\begin{array}{l}0.1383 \\
0.1359\end{array}$ & $\begin{array}{l}2.3186 \\
2.3093\end{array}$ & $\begin{array}{l}-2.198 \\
-2.141\end{array}$ & $\begin{array}{c}6.3254 \\
(2 ; 13) \\
6.3262 \\
(1 ; 13)\end{array}$ & $\begin{array}{c}{[\mathrm{Ja} 89]} \\
{[\mathrm{Po} 79]} \\
{[\mathrm{Ma92a}]}\end{array}$ \\
\hline${ }^{147} \mathrm{Sm}$ & $\begin{array}{l}1.565 \\
1.056\end{array}$ & .5 .9445 & 0.501 & 0.118 & 4.983 & $\begin{array}{l}0.1378 \\
0.1378\end{array}$ & $\begin{array}{l}2.3191 \\
2.2862\end{array}$ & $\begin{array}{l}-2.215 \\
-2.158\end{array}$ & $\begin{array}{l}6.3741 \\
(1 ; 14) \\
6.3748 \\
(1 ; 13)\end{array}$ & $\begin{array}{c}{[\mathrm{Ja} 89]} \\
{[\mathrm{Ba} 81]} \\
{[\mathrm{Ma} 92 \mathrm{a}]}\end{array}$ \\
\hline${ }^{148} \mathrm{Sm}$ & $\begin{array}{l}1.653 \\
1.565\end{array}$ & 6.0079 & 0.473 & 0.127 & 4.994 & $\begin{array}{l}0.1345 \\
0.1357\end{array}$ & $\begin{array}{l}2.2947 \\
2.3123\end{array}$ & $\begin{array}{l}-2.224 \\
-2.166\end{array}$ & $\begin{array}{c}6.3942 \\
(1 ; 13) \\
6.3947 \\
(1 ; 12)\end{array}$ & $\begin{array}{c}\text { [Ja89] } \\
{[\mathrm{Po} 79]} \\
{[\mathrm{Ma92a}]}\end{array}$ \\
\hline${ }^{149} \mathrm{Sm}$ & $\begin{array}{l}0.753 \\
1.530\end{array}$ & 5.9814 & 0.492 & 0.151 & 5.008 & $\begin{array}{l}0.1357 \\
0.1371\end{array}$ & $\begin{array}{l}2.3042 \\
2.3246\end{array}$ & $\begin{array}{l}-2.227 \\
-2.169\end{array}$ & $\begin{array}{c}6.4069 \\
(1 ; 13) \\
6.4076 \\
(1 ; 12)\end{array}$ & $\begin{array}{c}{[\mathrm{Ja} 89]} \\
{[\mathrm{Ba} 81]} \\
{[\mathrm{Ma} 92 \mathrm{a}]}\end{array}$ \\
\hline${ }^{150} \mathrm{Sm}$ & $\begin{array}{l}1.270 \\
0.207\end{array}$ & 5.8560 & 0.557 & 0.231 & 5.047 & $\begin{array}{l}0.1250 \\
0.1250\end{array}$ & $\begin{array}{l}2.2206 \\
2.2297\end{array}$ & $\begin{array}{l}-2.245 \\
-2.185\end{array}$ & $\begin{array}{c}6.4364 \\
(9 ; 12) \\
6.4374 \\
(9 ; 12)\end{array}$ & $\begin{array}{c}{[\mathrm{Ya} 78]} \\
{[\mathrm{Ma92a}]}\end{array}$ \\
\hline${ }^{152} \mathrm{Sm}$ & $\begin{array}{r}1.403 \\
-24.220\end{array}$ & 5.8688 & 0.554 & 0.297 & 5.092 & $\begin{array}{l}0.1332 \\
0.1322\end{array}$ & $\begin{array}{l}2.2884 \\
2.2893\end{array}$ & $\begin{array}{l}-2.248 \\
-2.188\end{array}$ & $\begin{array}{c}6.4891 \\
(1 ; 14) \\
6.4902 \\
(1 ; 23)\end{array}$ & $\begin{array}{c}{[\mathrm{Ja} 89]} \\
{[\mathrm{Po} 79]} \\
{[\mathrm{Ma} 92 \mathrm{a}]}\end{array}$ \\
\hline${ }^{154} \mathrm{Sm}$ & & 5.3601 & 0.4984 & 0.328 & 5.113 & & & & $\begin{array}{c}6.5215 \\
(29)^{\star}\end{array}$ & $\begin{array}{l}{[\text { Po79] }} \\
{[\text { Ja89] }}\end{array}$ \\
\hline
\end{tabular}

$\dagger$ The other Nd-isotopes can be found in TABLE III A.

* The given value is the total error including experimental and theoretical uncertainties 
TABLE IIIC. Barrett Radii and Related Parameters of Deformed Nuclei with $60 \leqslant Z \leqslant 77$ See page 194 for Explanation of Tables

\begin{tabular}{|c|c|c|c|c|c|c|c|c|c|c|}
\hline Isotope & $\begin{array}{l}\text { NPol } \\
{[\mathrm{keV}]}\end{array}$ & $\begin{array}{c}R_{\mathbf{0}} \\
{[\mathbf{f m}]}\end{array}$ & $\stackrel{\mathbf{a}}{[\mathbf{f m}]}$ & $\boldsymbol{\beta}_{\mathbf{2}}$ & $\begin{array}{c}\left\langle r^{2}\right\rangle_{\text {model }}^{1 / 2} \\
{[\mathbf{f m}]}\end{array}$ & $\begin{array}{c}\alpha \\
{[1 / f m]}\end{array}$ & $\mathbf{k}$ & $\begin{array}{c}C_{\mathrm{z}} \\
{[\mathrm{am} / \mathrm{keV}]}\end{array}$ & $\begin{array}{l}R_{\mathbf{m \alpha}}^{\mu} \\
{[\mathbf{f m}]}\end{array}$ & Ref. \\
\hline${ }^{151} \mathrm{Eu}$ & $\begin{array}{l}0.216 \\
1.185\end{array}$ & 6.0374 & 0.496 & 0.120 & 5.044 & $\begin{array}{l}0.1358 \\
0.1373\end{array}$ & $\begin{array}{l}2.3005 \\
2.3219\end{array}$ & $\begin{array}{l}-2.171 \\
-2.112\end{array}$ & $\begin{array}{c}6.4531 \\
(1 ; 13) \\
6.4539 \\
(1 ; 12)\end{array}$ & $\begin{array}{c}\text { [Ja89] } \\
{[\mathrm{Ta} 84 \mathrm{~b}]}\end{array}$ \\
\hline${ }^{153} \mathrm{Eu}$ & $\begin{array}{r}-11.754 \\
3.636\end{array}$ & 5.9350 & 0.527 & 0.320 & 5.118 & $\begin{array}{l}0.1362 \\
0.1333\end{array}$ & $\begin{array}{l}2.3071 \\
2.9235\end{array}$ & $\begin{array}{l}-2.190 \\
-2.130\end{array}$ & $\begin{array}{c}6.5258 \\
(1 ; 18) \\
6.5268 \\
(1 ; 13)\end{array}$ & $\begin{array}{c}{[\mathrm{Ja} 89]} \\
{[\mathrm{Ta} 84 \mathrm{~b}]}\end{array}$ \\
\hline${ }^{154} G d$ & $\begin{array}{l}-17.039 \\
-20.926\end{array}$ & 5.9625 & 0.531 & 0.290 & 5.122 & $\begin{array}{l}0.1357 \\
0.1337\end{array}$ & $\begin{array}{l}2.2946 \\
2.2873\end{array}$ & $\begin{array}{l}-2.125 \\
-2.066\end{array}$ & $\begin{array}{c}6.5322 \\
(7 ; 20) \\
6.5332 \\
(7 ; 21)\end{array}$ & $\begin{array}{c}{[\mathrm{Ja} 89]} \\
{[\mathrm{La83}]} \\
{[\mathrm{Ma92a}]}\end{array}$ \\
\hline${ }^{155} \mathrm{Gd}$ & $\begin{array}{r}-17.604 \\
18.839\end{array}$ & 6.0196 & 0.493 & 0.305 & 5.130 & $\begin{array}{l}0.1400 \\
0.1399\end{array}$ & $\begin{array}{l}2.3319 \\
2.3417\end{array}$ & $\begin{array}{l}-2.130 \\
-2.071\end{array}$ & $\begin{array}{c}6.5475 \\
(3 ; 20) \\
6.5485 \\
(2 ; 19)\end{array}$ & $\begin{array}{c}{[\mathrm{Be} 92]} \\
{[\mathrm{Ja} 89]} \\
{[\mathrm{La} 83]} \\
{[\mathrm{Ma} 92 \mathrm{a}]}\end{array}$ \\
\hline${ }^{186} G d$ & $\begin{array}{r}-26.810 \\
32.342\end{array}$ & 6.0094 & 0.510 & 0.310 & 5.142 & $\begin{array}{l}0.1374 \\
0.1340\end{array}$ & $\begin{array}{l}2.3111 \\
2.2928\end{array}$ & $\begin{array}{l}-2.134 \\
-2.074\end{array}$ & $\begin{array}{c}6.5607 \\
(1 ; 24) \\
6.5617 \\
(1 ; 24)\end{array}$ & $\begin{array}{c}{[\mathrm{Ja89}]} \\
{[\mathrm{La83}]} \\
{[\mathrm{Ma92a}]}\end{array}$ \\
\hline${ }^{157} \mathrm{Gd}$ & $\begin{array}{r}-20.113 \\
20.681\end{array}$ & 6.0100 & 0.501 & 0.323 & 5.146 & $\begin{array}{l}0.1350 \\
0.1362\end{array}$ & $\begin{array}{l}2.2917 \\
2.3118\end{array}$ & $\begin{array}{l}-2.136 \\
-2.077\end{array}$ & $\begin{array}{c}6.5653 \\
(3 ; 21) \\
6.5664 \\
(3 ; 20)\end{array}$ & $\begin{array}{c}{[\mathrm{Be} 92]} \\
{[\mathrm{Ja} 89]} \\
{[\mathrm{La} 83]} \\
{[\mathrm{Ma} 92 \mathrm{a}]}\end{array}$ \\
\hline${ }^{158} G d$ & $\begin{array}{r}-27.629 \\
30.249\end{array}$ & 6.0229 & 0.509 & 0.320 & 5.159 & $\begin{array}{l}0.1365 \\
0.1323\end{array}$ & $\begin{array}{l}2.3096 \\
2.2798\end{array}$ & $\begin{array}{l}-2.141 \\
-2.081\end{array}$ & $\begin{array}{c}6.5807 \\
(1 ; 25) \\
6.5817 \\
(1 ; 23)\end{array}$ & $\begin{array}{c}{[\mathrm{Ja} 89]} \\
{[\mathrm{La83}]} \\
{[\mathrm{Ma} 2 \mathrm{a}]}\end{array}$ \\
\hline${ }^{180} G d$ & $\begin{array}{r}-28.926 \\
29.932\end{array}$ & 6.0606 & 0.493 & 0.330 & 5.174 & $\begin{array}{l}0.1319 \\
0.1329\end{array}$ & $\begin{array}{l}2.2678 \\
2.2868\end{array}$ & $\begin{array}{l}-2.149 \\
-2.088\end{array}$ & $\begin{array}{c}6.6018 \\
(1 ; 25) \\
6.6029 \\
(1 ; 23)\end{array}$ & $\begin{array}{c}{[\mathrm{Ja} 89]} \\
{[\mathrm{La83}]} \\
{[\mathrm{Ma92a}]}\end{array}$ \\
\hline${ }^{150} \mathrm{~Tb}$ & & & & & & & & & & [Ta84a] \\
\hline
\end{tabular}


TABLE IIIC. Barrett Radii and Related Parameters of Deformed Nuclei with $60 \leqslant Z \leqslant 77$ See page 194 for Explanation of Tables

\begin{tabular}{|c|c|c|c|c|c|c|c|c|c|c|}
\hline Isotope & $\begin{array}{l}\text { NPol } \\
\text { [keV] }\end{array}$ & $\begin{array}{c}\boldsymbol{R}_{\mathbf{0}} \\
{[\mathbf{f m}]}\end{array}$ & $\stackrel{a}{\mathrm{f} m]}$ & $\boldsymbol{\beta}_{2}$ & $\begin{array}{c}\left\langle r^{2}\right\rangle_{\text {model }}^{1 / 2} \\
{[\mathrm{fm}]}\end{array}$ & $\begin{array}{c}\alpha \\
{[1 / \mathbf{f m}]}\end{array}$ & $\mathbf{k}$ & $\begin{array}{c}C_{\mathbf{z}} \\
{[\mathrm{am} / \mathrm{keV}]}\end{array}$ & $\begin{array}{l}R_{k \alpha}^{\mu} \\
{[\mathbf{f m}]}\end{array}$ & Ref. \\
\hline${ }^{101} D y$ & $\begin{array}{r}-26.834 \\
24.533\end{array}$ & 6.0375 & 0.523 & 0.321 & 5.196 & $\begin{array}{l}0.1385 \\
0.1390\end{array}$ & $\begin{array}{l}2.3059 \\
2.3218\end{array}$ & $\begin{array}{l}-2.027 \\
-1.966\end{array}$ & $\begin{array}{c}6.6224 \\
(5 ; 22) \\
6.6237 \\
(5 ; 21)\end{array}$ & [Be92] \\
\hline${ }^{102} D y$ & $\begin{array}{r}-29.695 \\
29.713\end{array}$ & 6.0415 & 0.523 & 0.334 & 5.209 & $\begin{array}{l}0.1385 \\
0.1378\end{array}$ & $\begin{array}{l}2.3069 \\
2.3123\end{array}$ & $\begin{array}{l}-2.032 \\
-1.971\end{array}$ & $\begin{array}{c}6.6368 \\
(1 ; 24) \\
6.6380 \\
(1 ; 23)\end{array}$ & [Be92] \\
\hline${ }^{103} D y$ & $\begin{array}{r}-24.686 \\
31.232\end{array}$ & 6.0438 & 0.523 & 0.334 & 5.211 & $\begin{array}{l}0.1387 \\
0.1382\end{array}$ & $\begin{array}{l}2.3095 \\
2.3171\end{array}$ & $\begin{array}{l}-2.033 \\
-1.972\end{array}$ & $\begin{array}{c}6.6426 \\
(2 ; 22) \\
6.6439 \\
(2 ; 23)\end{array}$ & [Be92] \\
\hline${ }^{164} D y$ & $\begin{array}{r}-30.578 \\
28.477\end{array}$ & 6.0584 & 0.523 & 0.338 & 5.224 & $\begin{array}{l}0.1383 \\
0.1385\end{array}$ & $\begin{array}{l}2.3075 \\
2.3206\end{array}$ & $\begin{array}{l}-2.037 \\
-1.976\end{array}$ & $\begin{array}{c}6.6562 \\
(4 ; 25) \\
6.6574 \\
(3 ; 22)\end{array}$ & [Be92] \\
\hline${ }^{165} \mathrm{Ho}$ & & & & & & & & & & [Po76] \\
\hline${ }^{166} \mathrm{Er}$ & $\begin{array}{r}-22.036 \\
22.183\end{array}$ & 6.0978 & 0.523 & 0.340 & 5.250 & $\begin{array}{l}0.1429 \\
0.1395\end{array}$ & $\begin{array}{l}2.3309 \\
2.3135\end{array}$ & $\begin{array}{l}-1.934 \\
-1.873\end{array}$ & $\begin{array}{c}6.6902 \\
(4 ; 20) \\
6.6914 \\
(4 ; 18)\end{array}$ & [Ma92a] \\
\hline${ }^{167} \mathrm{Er}$ & $\begin{array}{r}-23.505 \\
17.273\end{array}$ & 6.1075 & 0.523 & 0.341 & 5.258 & $\begin{array}{l}0.1415 \\
0.1390\end{array}$ & $\begin{array}{l}2.3191 \\
2.3094\end{array}$ & $\begin{array}{l}-1.937 \\
-1.875\end{array}$ & $\begin{array}{l}6.6977 \\
(7 ; 21) \\
6.6989 \\
(5 ; 16)\end{array}$ & [Ma92a] \\
\hline${ }^{168} E r$ & $\begin{array}{r}-22.404 \\
22.345\end{array}$ & 6.1208 & 0.523 & 0.346 & 5.272 & $\begin{array}{l}0.1410 \\
0.1400\end{array}$ & $\begin{array}{l}2.3158 \\
2.3190\end{array}$ & $\begin{array}{l}-1.941 \\
-1.878\end{array}$ & $\begin{array}{c}6.7086 \\
(5 ; 20) \\
6.7098 \\
(5 ; 18)\end{array}$ & [Ma92a] \\
\hline${ }^{170} \mathrm{Er}$ & $\begin{array}{r}-21.957 \\
21.801\end{array}$ & 6.1435 & 0.523 & 0.342 & 5.286 & $\begin{array}{l}0.1413 \\
0.1380\end{array}$ & $\begin{array}{l}2.3202 \\
2.3044\end{array}$ & $\begin{array}{l}-1.947 \\
-1.885\end{array}$ & $\begin{array}{c}6.7285 \\
(6 ; 20) \\
6.7297 \\
(4 ; 18)\end{array}$ & [Ma92a] \\
\hline
\end{tabular}


TABLE IIIC. Barrett Radii and Related Parameters of Deformed Nuclei with $60 \leqslant Z \leqslant 77$ See page 194 for Explanation of Tables

\begin{tabular}{|c|c|c|c|c|c|c|c|c|c|c|}
\hline Isotope & $\begin{array}{l}\text { NPol } \\
{[\mathrm{keV}]}\end{array}$ & $\begin{array}{c}R_{0} \\
{[\mathbf{f m}]}\end{array}$ & $\stackrel{a}{[\mathbf{f m}]}$ & $\beta_{2}$ & $\begin{array}{c}\left\langle r^{2}\right\rangle_{\text {model }}^{1 / 2} \\
{[\mathbf{f m}]}\end{array}$ & $\begin{array}{c}\alpha \\
{[1 / f m]}\end{array}$ & $\mathbf{k}$ & $\begin{array}{c}C_{z} \\
{[\mathrm{am} / \mathrm{keV}]}\end{array}$ & $\begin{array}{l}R_{k \alpha}^{\mu} \\
{[\mathbf{f m}]}\end{array}$ & Ref. \\
\hline${ }^{170} Y b$ & & 6.212 & 0.496 & 0.324 & 5.286 & & & & & [Ze75] \\
\hline${ }^{171} Y b$ & & 6.214 & 0.496 & 0.330 & 5.293 & & & & & [Ze75] \\
\hline${ }^{172} Y b$ & & 6.227 & 0.496 & 0.328 & 5.301 & & & & & [Ze75] \\
\hline${ }^{175} Y b$ & & 6.234 & 0.496 & 0.328 & 5.306 & & & & & [Ze75] \\
\hline${ }^{174} Y b$ & $\begin{array}{r}-35.397 \\
30.171\end{array}$ & 6.1999 & 0.523 & 0.321 & 5.317 & $\begin{array}{l}0.1444 \\
0.1405\end{array}$ & $\begin{array}{l}2.3327 \\
2.3115\end{array}$ & $\begin{array}{l}-1.858 \\
-1.794\end{array}$ & $\begin{array}{c}6.7733 \\
(1 ; 25) \\
6.7746 \\
(1 ; 21)\end{array}$ & $\begin{array}{l}{[\mathrm{Be} 92]} \\
{[\mathrm{Ze} 75]}\end{array}$ \\
\hline${ }^{176} Y b$ & & 6.271 & 0.496 & 0.313 & 5.321 & & & & & [Ze75] \\
\hline${ }^{176} \mathrm{Hf}$ & & 6.2880 & 0.519 & 0.270 & 5.331 & $\begin{array}{l}0.1388 \\
0.1388\end{array}$ & $\begin{array}{l}2.2740 \\
2.2847\end{array}$ & $\begin{array}{l}-1.775 \\
-1.710\end{array}$ & $\begin{array}{c}6.7990 \\
(9 ; 11) \\
6.8001 \\
(9 ; 10)\end{array}$ & [Ta84c] \\
\hline${ }^{177} H f$ & & 6.2970 & 0.517 & 0.263 & 5.334 & $\begin{array}{l}0.1388 \\
0.1388\end{array}$ & $\begin{array}{l}2.2745 \\
2.2852\end{array}$ & $\begin{array}{l}-1.777 \\
-1.711\end{array}$ & $\begin{array}{c}6.8030 \\
(9 ; 11) \\
6.8040 \\
(9 ; 10)\end{array}$ & [Ta84c] \\
\hline${ }^{178} H f$ & & 6.3170 & 0.514 & 0.259 & 5.338 & $\begin{array}{l}0.1388 \\
0.1388\end{array}$ & $\begin{array}{l}2.2753 \\
2.2859\end{array}$ & $\begin{array}{l}-1.779 \\
-1.714\end{array}$ & $\begin{array}{c}6.8107 \\
(9 ; 11) \\
6.8117 \\
(9 ; 10)\end{array}$ & [Ta84c] \\
\hline${ }^{178} \mathrm{Hf}$ & & 6.3480 & 0.498 & 0.257 & 5.339 & $\begin{array}{l}0.1388 \\
0.1388\end{array}$ & $\begin{array}{l}2.2762 \\
2.2868\end{array}$ & $\begin{array}{l}-1.782 \\
-1.716\end{array}$ & $\begin{array}{c}6.8162 \\
(9 ; 11) \\
6.8172 \\
(9 ; 10)\end{array}$ & [Ta84c] \\
\hline${ }^{180} \mathrm{Hf}$ & $\begin{array}{r}-29.536 \\
29.851\end{array}$ & 6.3177 & 0.510 & 0.276 & 5.349 & $\begin{array}{l}0.1457 \\
0.1440\end{array}$ & $\begin{array}{l}2.3305 \\
2.3286\end{array}$ & $\begin{array}{l}-1.781 \\
-1.715\end{array}$ & $\begin{array}{c}6.8218 \\
(2 ; 22) \\
6.8231 \\
(2 ; 20)\end{array}$ & $\begin{array}{l}{[\mathrm{Be} 92]} \\
{[\mathrm{Ta} 84 \mathrm{c}]}\end{array}$ \\
\hline
\end{tabular}


TABLE IIIC. Barrett Radii and Related Parameters of Deformed Nuclei with $60 \leqslant Z \leqslant 77$ See page 194 for Explanation of Tables

\begin{tabular}{|c|c|c|c|c|c|c|c|c|c|c|}
\hline Isotope & $\begin{array}{l}\text { NPol } \\
{[\mathrm{keV}]}\end{array}$ & $\begin{array}{c}R_{0} \\
{[\mathbf{f m}]}\end{array}$ & $\stackrel{\mathbf{a}}{[\mathrm{fm}]}$ & $\beta_{2}$ & $\begin{array}{c}\left\langle r^{2}\right\rangle_{\text {model }}^{1 / 2} \\
{[\mathbf{f m}]}\end{array}$ & $\underset{[1 / f m]}{\alpha}$ & $\mathbf{k}$ & $\begin{array}{c}C_{*} \\
{[\mathrm{am} / \mathrm{keV}]}\end{array}$ & $\begin{array}{l}R_{k \alpha}^{\mu} \\
{[\mathbf{f m}]}\end{array}$ & Ref. \\
\hline${ }^{181} \mathrm{Ta}$ & $\begin{array}{r}-20.175 \\
0.707\end{array}$ & 6.3669 & 0.494 & 0.273 & 5.354 & $\begin{array}{l}0.1462 \\
0.1468\end{array}$ & $\begin{array}{l}2.3274 \\
2.3447\end{array}$ & $\begin{array}{l}-1.742 \\
-1.676\end{array}$ & $\begin{array}{l}6.8343 \\
(2 ; 18) \\
6.8354 \\
(2 ; 18)\end{array}$ & $\begin{array}{l}{[\mathrm{Be} 92]} \\
{[\mathrm{Po} 77]}\end{array}$ \\
\hline${ }^{182} W$ & $\begin{array}{r}-25.356 \\
27.426\end{array}$ & 6.3338 & 0.523 & 0.260 & 5.364 & $\begin{array}{l}0.1464 \\
0.1441\end{array}$ & $\begin{array}{l}2.3198 \\
2.3143\end{array}$ & $\begin{array}{l}-1.700 \\
-1.635\end{array}$ & $\begin{array}{l}6.8427 \\
(4 ; 19) \\
6.8440 \\
(4 ; 18)\end{array}$ & [Ma92a] \\
\hline${ }^{184} W$ & $\begin{array}{r}-23.402 \\
28.962\end{array}$ & 6.3599 & 0.523 & 0.244 & 5.373 & $\begin{array}{l}0.1483 \\
0.1451\end{array}$ & $\begin{array}{l}2.3577 \\
2.3235\end{array}$ & $\begin{array}{l}-1.704 \\
-1.638\end{array}$ & $\begin{array}{c}6.8562 \\
(4 ; 18) \\
6.8575 \\
(4 ; 18)\end{array}$ & [Ma92a] \\
\hline${ }^{180} W$ & $\begin{array}{r}-22.398 \\
31.192\end{array}$ & 6.3839 & 0.523 & 0.229 & 5.381 & $\begin{array}{l}0.1465 \\
0.1456\end{array}$ & $\begin{array}{l}2.3236 \\
2.3296\end{array}$ & $\begin{array}{l}-1.708 \\
-1.642\end{array}$ & $\begin{array}{l}6.8683 \\
(3 ; 18) \\
6.8696 \\
(4 ; 19)\end{array}$ & [Ma92a] \\
\hline 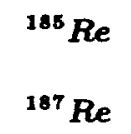 & & & & & & & & & & $\begin{array}{l}{[\mathrm{Ko81}]} \\
{[\mathrm{Ko81}]}\end{array}$ \\
\hline${ }^{186} \mathrm{Os}$ & $\begin{array}{l}3.924 \\
4.102\end{array}$ & 6.4142 & 0.522 & 0.200 & 5.387 & $\begin{array}{l}0.1445 \\
0.1445\end{array}$ & $\begin{array}{l}2.2894 \\
2.3030\end{array}$ & $\begin{array}{l}-1.633 \\
-1.566\end{array}$ & $\begin{array}{l}6.8766 \\
(11 ; 19) \\
6.8779 \\
(10 ; 19)\end{array}$ & [Ho81] \\
\hline${ }^{188} \mathrm{Os}$ & $\begin{array}{l}3.733 \\
4.072\end{array}$ & 6.4268 & 0.528 & 0.185 & 5.395 & $\begin{array}{l}0.1445 \\
0.1445\end{array}$ & $\begin{array}{l}2.2892 \\
2.3040\end{array}$ & $\begin{array}{l}-1.636 \\
-1.569\end{array}$ & $\begin{array}{l}6.8888 \\
(11 ; 18) \\
6.8902 \\
(9 ; 19)\end{array}$ & [Ho81] \\
\hline
\end{tabular}


G. FRICKE et al. Nuclear Charge Radii

TABLE IIIC. Barrett Radii and Related Parameters of Deformed Nuclei with $60 \leqslant Z \leqslant 77$ See page 194 for Explanation of Tables

\begin{tabular}{|c|c|c|c|c|c|c|c|c|c|c|}
\hline Isotope & $\begin{array}{l}\text { NPol } \\
\text { [keV] }\end{array}$ & $\begin{array}{c}\boldsymbol{R}_{\mathbf{0}} \\
{[\mathbf{f m}]}\end{array}$ & $\underset{[\mathbf{f m}]}{a}$ & $\boldsymbol{\beta}_{2}$ & $\begin{array}{c}\left\langle r^{2}\right\rangle_{\text {model }}^{1 / 2} \\
{[\mathrm{fm}]}\end{array}$ & $\stackrel{\alpha}{[1 / f m]}$ & $\mathbf{k}$ & $\begin{array}{c}C_{z} \\
{[\mathrm{am} / \mathrm{keV}]}\end{array}$ & $\begin{array}{l}\boldsymbol{R}_{\mathrm{mo}}^{\mu} \\
\text { [fm] }\end{array}$ & Ref. \\
\hline${ }^{180} \mathrm{Os}$ & $\begin{array}{l}3.519 \\
3.744\end{array}$ & 6.4555 & 0.522 & 0.170 & 5.401 & $\begin{array}{l}0.1445 \\
0.1445\end{array}$ & $\begin{array}{l}2.2917 \\
2.3052\end{array}$ & $\begin{array}{r}-1.640 \\
-1.572\end{array}$ & $\begin{array}{c}6.8995 \\
(9 ; 17) \\
6.9008 \\
(8 ; 18)\end{array}$ & [Ho81] \\
\hline${ }^{192} \mathrm{Os}$ & $\begin{array}{l}3.376 \\
3.564\end{array}$ & 6.4829 & 0.511 & 0.170 & 5.406 & $\begin{array}{l}0.1445 \\
0.1445\end{array}$ & $\begin{array}{l}2.2930 \\
2.3065\end{array}$ & $\begin{array}{l}-1.644 \\
-1.576\end{array}$ & $\begin{array}{c}6.9097 \\
(9 ; 17) \\
6.9108 \\
(8 ; 17)\end{array}$ & [Ho81] \\
\hline $\begin{array}{l}{ }^{101} I r \\
{ }^{103} I r\end{array}$ & & & & & & & & & & $\begin{array}{l}{[\mathrm{Ta} 84 \mathrm{a}} \\
{[\mathrm{Ta} 84 \mathrm{a}}\end{array}$ \\
\hline
\end{tabular}


TABLE IV. Confit Matrices for Muonic $2 p \rightarrow 1 s$ Energy Differences of Isotopes, $31 \leqslant Z \leqslant 58$ See page 194 for Explanation of Tables

\begin{tabular}{|c|rrrrr|}
\hline$[\mathrm{keV}]$ & ${ }^{70} \mathrm{Ge}$ & ${ }^{72} \mathrm{Ge}$ & ${ }^{73} \mathrm{Ge}$ & ${ }^{74} \mathrm{Ge}$ & ${ }^{76} \mathrm{Ge}$ \\
\hline${ }^{70} \mathrm{Ge}$ & & & & & \\
& & 2.024 & 2.861 & 4.130 & 5.080 \\
${ }^{72} \mathrm{Ge}$ & -2.031 & & 0.837 & 2.106 & 3.056 \\
& 15 & & 35 & 28 & 26 \\
${ }^{73} \mathrm{Ge}$ & -2.829 & -0.798 & & 1.269 & 2.219 \\
& 25 & 23 & & 41 & 40 \\
${ }^{74} \mathrm{Ge}$ & -4.192 & -2.161 & -1.368 & & 0.950 \\
& 21 & 16 & 28 & & 31 \\
${ }^{76} \mathrm{Ge}$ & -5.128 & -3.097 & -2.299 & -0.936 & \\
& 20 & 14 & 26 & 18 & \\
\hline
\end{tabular}

\begin{tabular}{|l|rrrrr|}
\hline$[\mathrm{keV}]$ & ${ }^{70} \mathrm{Se}$ & ${ }^{77} \mathrm{Se}$ & ${ }^{78} \mathrm{Se}$ & ${ }^{80} \mathrm{Se}$ & ${ }^{82} \mathrm{Se}$ \\
\hline \hline${ }^{70} \mathrm{Se}$ & & 0.268 & 0.269 & 0.262 & 0.267 \\
& & 26 & 25 & 23 & 28 \\
${ }^{77} \mathrm{Se}$ & -0.203 & & 0.001 & -0.006 & 0.001 \\
& 18 & & 25 & 22 & 29 \\
${ }^{78} \mathrm{Se}$ & -0.254 & -0.050 & & -0.007 & -0.002 \\
& 18 & 19 & & 21 & 25 \\
${ }^{80} \mathrm{Se}$ & -0.216 & -0.013 & 0.038 & & 0.005 \\
& 16 & 16 & 16 & & 26 \\
${ }^{\circ 2} \mathrm{Se}$ & -0.254 & -0.050 & -0.000 & -0.038 & \\
& 20 & 23 & 19 & 20 & \\
\hline \hline
\end{tabular}


TABLE IV. Confit Matrices for Muonic $2 p \rightarrow 1 s$ Energy Differences of Isotopes, $31 \leqslant Z \leqslant 58$ See page 194 for Explanation of Tables

\begin{tabular}{|l|rr|}
\hline$[\mathrm{keV}]$ & ${ }^{79} \mathrm{Br}$ & ${ }^{81} \mathrm{Br}$ \\
\hline${ }^{79} \mathrm{Br}$ & & -0.468 \\
& & 30 \\
${ }^{\mathrm{a} 1} \mathrm{Br}$ & 0.462 & \\
& 25 & \\
\hline
\end{tabular}

\begin{tabular}{|c|c|c|c|c|c|c|}
\hline [keV] & ${ }^{78} \mathrm{Kr}$ & ${ }^{80} K r$ & ${ }^{82} K r$ & ${ }^{83} K r$ & ${ }^{84} K r$ & ${ }^{86} \mathrm{Kr}$ \\
\hline \multirow[t]{2}{*}{${ }^{78} \mathrm{Kr}$} & & -0.922 & -1.779 & -2.684 & -2.489 & -3.274 \\
\hline & & 47 & 46 & 55 & 44 & 39 \\
\hline \multirow[t]{2}{*}{${ }^{80} K r$} & 0.911 & & -0.857 & -1.762 & -1.567 & -2.352 \\
\hline & 39 & & 47 & 55 & 45 & 39 \\
\hline \multirow[t]{2}{*}{${ }^{82} K r$} & 1.796 & 0.885 & & -0.905 & -0.710 & -1.495 \\
\hline & 39 & 39 & & 55 & 44 & 39 \\
\hline \multirow[t]{2}{*}{${ }^{83} K r$} & 2.928 & 2.017 & 1.132 & & 0.195 & -0.590 \\
\hline & 47 & 47 & 47 & & 53 & 48 \\
\hline \multirow[t]{2}{*}{${ }^{84} \mathrm{Kr}$} & 2.394 & 1.483 & 0.598 & -0.5 .34 & & -0.785 \\
\hline & 38 & 38 & 38 & 46 & & 36 \\
\hline \multirow[t]{2}{*}{${ }^{86} \mathrm{Kr}$} & 3.318 & 2.407 & 1.522 & 0.390 & 0.924 & \\
\hline & 33 & 33 & 33 & 42 & 32 & \\
\hline
\end{tabular}

\begin{tabular}{|l|rr|}
\hline$[\mathrm{keV}]$ & ${ }^{85} R b$ & ${ }^{87} R b$ \\
\hline${ }^{85} R b$ & & -1.015 \\
& & 23 \\
${ }^{87} R b$ & 0.940 & \\
& 15 & \\
\hline
\end{tabular}


TABLE IV. Confit Matrices for Muonic $2 p \rightarrow 1 s$ Energy Differences of Isotopes, $31 \leqslant Z \leqslant 58$ See page 194 for Explanation of Tables

\begin{tabular}{|l|rrrr|}
\hline$[\mathrm{keV}]$ & ${ }^{84} \mathrm{Sr}$ & ${ }^{86} \mathrm{Sr}$ & ${ }^{87} \mathrm{Sr}$ & ${ }^{88} \mathrm{Sr}$ \\
\hline${ }^{84} \mathrm{Sr}$ & & -1.990 & -33.251 & -3.513 \\
& & 35 & 70 & 34 \\
${ }^{86} \mathrm{Sr}$ & 1.983 & & -1.260 & -1.522 \\
& 24 & & 64 & 15 \\
${ }^{87} \mathrm{Sr}$ & 3.332 & 1.349 & & -0.262 \\
& 54 & 50 & & 63 \\
${ }^{88} \mathrm{Sr}$ & 3.515 & 1.532 & 0.183 & \\
& 24 & 10 & 49 & \\
\hline \hline
\end{tabular}

\begin{tabular}{|l|rrrrr|}
\hline$[\mathrm{keV}]$ & ${ }^{90} \mathrm{Zr}$ & ${ }^{91} \mathrm{Zr}$ & ${ }^{92} \mathrm{Zr}$ & ${ }^{94} \mathrm{Zr}$ & ${ }^{96} \mathrm{Zr}$ \\
\hline \hline${ }^{90} \mathrm{Zr}$ & & 3.507 & 8.141 & 13.994 & 18.201 \\
& & 44 & 20 & 23 & 50 \\
${ }^{91} \mathrm{Zr}$ & -3.453 & & 4.634 & 10.487 & 14.693 \\
& 33 & & 46 & 47 & 65 \\
${ }^{02} \mathrm{Zr}$ & -8.232 & -4.779 & & 5.853 & 10.060 \\
& 14 & 34 & & 26 & 51 \\
${ }^{84} \mathrm{Zr}$ & -14.114 & -10.661 & -5.882 & & 4.206 \\
& 16 & 35 & 17 & & 52 \\
${ }^{90} \mathrm{Zr}$ & -18.419 & -14.966 & -10.187 & -4.305 & \\
& 36 & 48 & 36 & 37 & \\
\hline \hline
\end{tabular}


G. FRICKE et al. Nuclear Charge Radii

TABLE IV. Confit Matrices for Muonic $2 p \rightarrow 1 s$ Energy Differences of Isotopes, $31 \leqslant Z \leqslant 58$ See page 194 for Explanation of Tables

\begin{tabular}{|c|c|c|c|c|c|c|c|}
\hline$[\mathrm{keV}]$ & ${ }^{92} \mathrm{Mo}$ & ${ }^{94} \mathrm{Mo}$ & ${ }^{95} M o$ & ${ }^{80} M O$ & ${ }^{97} \mathrm{Mo}$ & ${ }^{98} \mathrm{Mo}$ & ${ }^{100} \mathrm{Mo}$ \\
\hline \multirow[t]{2}{*}{${ }^{02} \mathrm{Mo}$} & & 9.140 & 11.873 & 17.258 & 18.3336 & 23.457 & 32.782 \\
\hline & & 38 & 35 & 33 & 37 & 40 & 40 \\
\hline \multirow[t]{2}{*}{${ }^{84} \mathrm{Mo}$} & -9.310 & & 2.733 & 8.119 & 9.197 & 14.318 & 23.642 \\
\hline & 26 & & 27 & 24 & 29 & 33 & 33 \\
\hline \multirow[t]{2}{*}{${ }^{95} \mathrm{Mo}$} & -11.978 & -2.668 & & 5.385 & 6.463 & 11.584 & 20.909 \\
\hline & 25 & 19 & & 18 & 23 & 29 & 29 \\
\hline \multirow[t]{2}{*}{${ }^{96} \mathrm{Mo}$} & -17.412 & -8.102 & $-5.4: 34$ & & 1.078 & 6.199 & 15.523 \\
\hline & 23 & 17 & 14 & & 20 & 27 & 26 \\
\hline \multirow[t]{2}{*}{${ }^{97} \mathrm{Mo}$} & -18.379 & -9.069 & -6.401 & -0.967 & & 5.121 & 14.446 \\
\hline & 26 & 21 & 17 & 16 & & 31 & 30 \\
\hline \multirow[t]{2}{*}{${ }^{98} \mathrm{Mo}$} & -23.676 & -14.366 & -11.698 & -6.264 & -5.298 & & 9.325 \\
\hline & 28 & 22 & 20 & 19 & 22 & & 35 \\
\hline \multirow[t]{2}{*}{${ }^{100} \mathrm{Mo}$} & -33.095 & -23.784 & -21.117 & -15.683 & -14.716 & -9.418 & \\
\hline & 27 & 22 & 20 & 18 & 22 & 23 & \\
\hline
\end{tabular}

\begin{tabular}{|c|c|c|c|c|c|c|c|}
\hline$[\mathrm{keV}]$ & ${ }^{96} R u$ & ${ }^{88} \mathrm{Ru}$ & ${ }^{90} R u$ & ${ }^{100} \mathrm{Ru}$ & ${ }^{101} R u$ & ${ }^{102} R u$ & ${ }^{104} R u$ \\
\hline \multirow[t]{2}{*}{${ }^{90} R u$} & & 9.051 & 12.324 & 17.604 & $20.29: 3$ & 25.489 & 33.626 \\
\hline & & 577 & 240 & 241 & 236 & 230 & 232 \\
\hline \multirow[t]{2}{*}{${ }^{98} R u$} & -8.868 & & 3.273 & 8.55 .3 & 11.242 & 16.438 & 24.575 \\
\hline & 232 & & 5.36 & 536 & 534 & 531 & 532 \\
\hline \multirow[t]{2}{*}{${ }^{99} \mathrm{Ru}$} & -12.436 & -3.568 & & 5.280 & 7.969 & 13.165 & 21.303 \\
\hline & 58 & 235 & & 110 & 100 & 84 & 89 \\
\hline \multirow[t]{2}{*}{${ }^{100} R u$} & $-17.69^{\circ} 2$ & -8.825 & -5.256 & & 2.689 & 7.885 & 16.023 \\
\hline & 68 & $\cdot 238$ & 78 & & 101 & 86 & 90 \\
\hline \multirow[t]{2}{*}{${ }^{101} R u$} & .20 .106 & -11.238 & -7.670 & -2.414 & & 5.196 & 13.333 \\
\hline & 106 & 252 & 113 & 119 & & 71 & 77 \\
\hline \multirow[t]{2}{*}{${ }^{102} R u$} & -25.76 .3 & -16.895 & -13.326 & -8.070 & -5.656 & & 8.137 \\
\hline & 40 & $2: 32$ & 56 & 67 & 106 & & 55 \\
\hline \multirow[t]{2}{*}{${ }^{104} R u$} & -34.012 & -25.144 & -21.576 & -16.319 & -13.906 & -8.249 & \\
\hline & 47 & 233 & 61 & 71 & 108 & 45 & \\
\hline
\end{tabular}


TABLE IV. Confit Matrices for Muonic $2 p \rightarrow 1 s$ Energy Differences of Isotopes, $31 \leqslant Z \leqslant 58$ See page 194 for Explanation of Tables

\begin{tabular}{|l|rrrrrr|}
\hline$[\mathrm{keV}]$ & ${ }^{102} \boldsymbol{P d}$ & ${ }^{104} \boldsymbol{P d}$ & ${ }^{105} \boldsymbol{P d}$ & ${ }^{106} \boldsymbol{P d}$ & ${ }^{108} \boldsymbol{P d}$ & ${ }^{110} \boldsymbol{P d}$ \\
\hline \hline \multirow{1}{102}{$\boldsymbol{P d}$} & & 7.975 & 11.040 & 15.492 & 23.027 & 29.933 \\
& & 176 & 150 & 132 & 130 & 176 \\
${ }^{104} \boldsymbol{P d}$ & -8.041 & & 3.066 & 7.517 & 15.052 & 21.958 \\
& 143 & & 173 & 158 & 156 & 196 \\
${ }^{105} \boldsymbol{P d}$ & -10.172 & -2.131 & & 4.451 & 11.987 & 18.893 \\
& 168 & 191 & & 127 & 125 & 172 \\
${ }^{106} \boldsymbol{P d}$ & -15.648 & -7.606 & -5.476 & & 7.535 & 14.441 \\
& 95 & 131 & 157 & & 103 & 157 \\
${ }^{108} \boldsymbol{P d}$ & -23.346 & -15.305 & -13.175 & -7.699 & & 6.906 \\
& 91 & 128 & 155 & 70 & & 155 \\
${ }^{110} \boldsymbol{P d}$ & -30.252 & -22.211 & -20.080 & -14.605 & -6.906 & \\
& 118 & 148 & 172 & 102 & 99 & \\
& & & & & & \\
\hline
\end{tabular}

\begin{tabular}{|l|rr|}
\hline$[\mathrm{keV}]$ & ${ }^{107} \mathrm{Ag}$ & ${ }^{100} \mathrm{Ag}$ \\
\hline${ }^{107} \mathrm{Ag}$ & & 7.074 \\
& & 39 \\
${ }^{100} \mathrm{Ag}$ & -7.178 & \\
& 27 & \\
\hline
\end{tabular}


TABLE IV. Confit Matrices for Muonic $2 p \rightarrow 1 s$ Energy Differences of Isotopes, $31 \leqslant Z \leqslant 58$ See page 194 for Explanation of Tables

\begin{tabular}{|c|c|c|c|c|c|c|c|c|}
\hline$[\mathrm{keV}]$ & ${ }^{100} C d$ & ${ }^{108} \mathrm{Cd}$ & ${ }^{110} C d$ & ${ }^{111} C d$ & ${ }^{112} C d$ & ${ }^{213} C d$ & ${ }^{114} C d$ & ${ }^{110} C d$ \\
\hline \multirow[t]{2}{*}{${ }^{100} \mathrm{Cd}$} & & 7.004 & 13.991 & 15.893 & 20.752 & 22.551 & 26.888 & 32.051 \\
\hline & & 129 & 118 & 124 & 114 & 120 & 113 & 114 \\
\hline \multirow[t]{2}{*}{${ }^{108} C d$} & -7.050 & & 6.987 & 8.889 & 13.748 & 15.547 & 19.884 & 25.047 \\
\hline & 86 & & 84 & 91 & 82 & 90 & 79 & 82 \\
\hline \multirow[t]{2}{*}{${ }^{110} C d$} & -14.077 & -7.027 & & 1.901 & 6.761 & 8.560 & 12.897 & 18.060 \\
\hline & 76 & 68 & & 69 & 58 & 68 & 55 & 58 \\
\hline \multirow[t]{2}{*}{${ }^{111} C d$} & -15.951 & -8.901 & -1.874 & & 4.860 & 6.658 & 10.995 & 16.159 \\
\hline & 77 & 69 & 49 & & 69 & 78 & 66 & 69 \\
\hline \multirow[t]{2}{*}{${ }^{112} C d$} & -20.860 & -13.811 & -6.784 & -4.910 & & 1.799 & 6.136 & 11.299 \\
\hline & 70 & 66 & 46 & 48 & & 59 & 47 & 49 \\
\hline \multirow[t]{2}{*}{${ }^{113} C d$} & -22.796 & -15.747 & -8.720 & -6.846 & -1.936 & & 4.3337 & 9.500 \\
\hline & 75 & 72 & 54 & 56 & 39 & & 59 & 59 \\
\hline \multirow[t]{2}{*}{${ }^{114} C d$} & -27.056 & -20.006 & -12.979 & -11.105 & -6.195 & -4.260 & & 5.163 \\
\hline & 70 & 66 & 46 & 48 & 36 & 44 & & 43 \\
\hline \multirow[t]{2}{*}{${ }^{116} \mathrm{Cd}$} & -32.301 & -25.251 & -18.224 & -16.350 & -11.441 & -9.505 & -5.245 & \\
\hline & 71 & 68 & 49 & 51 & 37 & 43 & 34 & \\
\hline
\end{tabular}

\begin{tabular}{|l|rr|}
\hline \hline$[\mathrm{keV}]$ & ${ }^{121} \mathrm{Sb}$ & ${ }^{123} \mathrm{Sb}$ \\
\hline${ }^{121} \mathrm{Sb}$ & & 3.429 \\
& & 96 \\
${ }^{123} \mathrm{Sb}$ & -3.187 & \\
& 77 & \\
\hline
\end{tabular}


G. FRICKE et al. Nuclear Charge Radii

TABLE IV. Confit Matrices for Muonic $2 p \rightarrow 1 s$ Energy Differences of Isotopes, $31 \leqslant Z \leqslant 58$ See page 194 for Explanation of Tables

\begin{tabular}{|c|c|c|c|c|c|c|c|}
\hline [keV] & ${ }^{122} \mathrm{Te}$ & ${ }^{123} \mathrm{Te}$ & ${ }^{124} \mathrm{Te}$ & ${ }^{125} \mathrm{Te}$ & ${ }^{126} \mathrm{Te}$ & ${ }^{128} \mathrm{Te}$ & ${ }^{130} \mathrm{Te}$ \\
\hline \multirow[t]{2}{*}{${ }^{123} \mathrm{Te}$} & & 1.242 & 3.992 & 4.898 & 7.484 & 10.778 & 13.839 \\
\hline & & 357 & 355 & 356 & 355 & 356 & 355 \\
\hline \multirow[t]{2}{*}{${ }^{123} \mathrm{Te}$} & -1.268 & & 2.749 & 3.655 & 6.241 & 9.5336 & 12.596 \\
\hline & 356 & & 38 & 43 & 38 & 41 & 38 \\
\hline \multirow[t]{2}{*}{${ }^{124} \mathrm{Te}$} & -4.015 & -2.747 & & 0.906 & 3.492 & 6.787 & 9.847 \\
\hline & 355 & 26 & & 35 & 27 & 31 & 27 \\
\hline \multirow[t]{2}{*}{${ }^{125} T e$} & -4.928 & -3.660 & -0.913 & & 2.586 & 5.881 & 8.941 \\
\hline & 355 & 29 & 24 & & 34 & 36 & 33 \\
\hline \multirow[t]{2}{*}{${ }^{126} \mathrm{Te}$} & -7.580 & -6.312 & -3.565 & -2.652 & & 3.294 & 6.355 \\
\hline & 355 & 27 & 19 & 24 & & 31 & 26 \\
\hline \multirow[t]{2}{*}{${ }^{128} \mathrm{Te}$} & -10.883 & -9.615 & -6.868 & -5.955 & -3.303 & & 3.061 \\
\hline & 355 & 29 & 22 & 25 & 22 & & 29 \\
\hline \multirow[t]{2}{*}{${ }^{130} \mathrm{Te}$} & -13.986 & -12.718 & -9.971 & -9.058 & -6.406 & -3.103 & \\
\hline & 355 & 29 & 21 & 25 & 20 & 22 & \\
\hline
\end{tabular}

\begin{tabular}{|c|c|c|c|c|c|c|c|c|c|}
\hline$[\mathrm{keV}]$ & ${ }^{124} X e$ & ${ }^{126} X e$ & ${ }^{128} \mathrm{Xe}$ & ${ }^{120} X e$ & ${ }^{130} X e$ & ${ }^{131} X e$ & ${ }^{132} X e$ & ${ }^{134} X e$ & ${ }^{136} X e$ \\
\hline \multirow[t]{2}{*}{${ }^{124} X e$} & & 3.765 & 6.079 & 6.530 & 9.421 & 8.605 & 10.928 & 13.448 & 16.709 \\
\hline & & 104 & 107 & 41 & 199 & 39 & 68 & 45 & 35 \\
\hline \multirow[t]{2}{*}{${ }^{120} X e$} & -3.510 & & 2.314 & 2.765 & 5.656 & 4.840 & 7.163 & 9.683 & 12.944 \\
\hline & 101 & & 142 & 102 & 220 & 101 & 116 & 104 & 100 \\
\hline \multirow[t]{2}{*}{${ }^{128} \mathrm{Xe}$} & -5.866 & -2.356 & & 0.451 & 3.341 & 2.526 & 4.849 & 7.369 & 10.630 \\
\hline & 104 & 142 & & 105 & 221 & 104 & 118 & 106 & 102 \\
\hline \multirow[t]{2}{*}{${ }^{128} X e$} & -6.224 & -2.714 & -0.358 & & 2.890 & 2.075 & 4.398 & 6.918 & 10.179 \\
\hline & 30 & 101 & 104 & & 198 & 33 & 65 & 41 & 28 \\
\hline \multirow[t]{2}{*}{${ }^{130} X e$} & -9.271 & -5.761 & -3.405 & -3.047 & & -0.815 & 1.508 & 4.028 & 7.288 \\
\hline & 198 & 220 & 221 & 198 & & 198 & 206 & 199 & 197 \\
\hline \multirow[t]{2}{*}{${ }^{131} X e$} & -8.432 & -4.922 & -2.566 & -2.208 & 0.839 & & $2.32: 3$ & 4.843 & 8.104 \\
\hline & 28 & 100 & 103 & 27 & 197 & & 64 & 38 & 24 \\
\hline \multirow[t]{2}{*}{${ }^{132} \mathrm{Xe}$} & -10.914 & -7.404 & -5.048 & -4.690 & -1.643 & -2.482 & & 2.520 & 5.781 \\
\hline & 45 & 106 & 109 & 44 & 201 & $4: 3$ & & 68 & 61 \\
\hline \multirow[t]{2}{*}{${ }^{134} X e$} & -13.351 & -9.840 & -7.484 & -7.126 & -4.079 & -4.918 & -2.436 & & 3.261 \\
\hline & 30 & 101 & 104 & 30 & 198 & 27 & 44 & & 34 \\
\hline \multirow[t]{2}{*}{${ }^{136} X e$} & -16.693 & -13.182 & -10.827 & -10.469 & -7.421 & -8.260 & -5.778 & -3.342 & \\
\hline & 23 & 99 & 102 & 23 & 197 & 19 & 40 & 23 & \\
\hline
\end{tabular}




\section{G. FRICKE et al. Nuclear Charge Radii}

TABLE IV. Confit Matrices for Muonic $2 p \rightarrow 1 s$ Energy Differences of Isotopes, $31 \leqslant Z \leqslant 58$ See page 194 for Explanation of Tables

\begin{tabular}{|l|rrrrr|}
\hline$[\mathrm{keV}]$ & ${ }^{134} \mathrm{Ba}$ & ${ }^{136} \mathrm{Ba}$ & ${ }^{136} \mathrm{Ba}$ & ${ }^{137} \mathrm{Ba}$ & ${ }^{138} \mathrm{Ba}$ \\
\hline \hline${ }^{134} \mathrm{Ba}$ & & -0.936 & 1.553 & 4.596 \\
& & 81 & 39 & 34 \\
${ }^{138} \mathrm{Ba}$ & 1.059 & & 2.489 & 5.532 \\
& 51 & & 78 & 76 \\
${ }^{136} \mathrm{Ba}$ & -1.590 & -2.649 & & 3.043 \\
& 28 & 45 & & 28 \\
${ }^{137} \mathrm{Ba}$ & $-1.29: 3$ & -2.352 & 0.297 & & \\
& 64 & 70 & 60 & & \\
${ }^{138} \mathrm{Ba}$ & -4.696 & -5.754 & -3.105 & -3.402 & \\
& 25 & 47 & 21 & 60 & \\
\hline
\end{tabular}

\begin{tabular}{|c|c|c|}
\hline [keV] & ${ }^{140} \mathrm{Ce}$ & ${ }^{142} \mathrm{Ce}$ \\
\hline${ }^{140} \mathrm{Ce}$ & $\begin{array}{r}-15.689 \\
86\end{array}$ & $\begin{array}{r}15.298 \\
127\end{array}$ \\
\hline
\end{tabular}


G. FRICKE et al. Nuclear Charge Radii

TABLE V. Differences of Barrett Radii for Isotopes, $6 \leqslant Z \leqslant 82$

See page 194 for Explanation of Tables

\begin{tabular}{|c|c|}
\hline Isotope Pair & $\Delta R_{k \alpha \alpha}^{\mu s}[a m]$ \\
\hline${ }^{13} C \leftrightarrow{ }^{12} C$ & $-2.9 \pm 16.6 \pm 1.1$ \\
\hline${ }^{14} C \leftrightarrow{ }^{12} C$ & $27.7 \pm 12.4 \pm 1.1$ \\
\hline${ }^{18} \mathrm{O} \leftrightarrow{ }^{16} \mathrm{O}$ & $98.6 \pm 7.8 \pm 0.7$ \\
\hline${ }^{21} \mathrm{Ne} \leftrightarrow{ }^{20} \mathrm{Ne}$ & $-49.3 \pm 3.1 \pm 1.0$ \\
\hline${ }^{22} \mathrm{Ne} \leftrightarrow{ }^{20} \mathrm{Ne}$ & $-67.0 \pm 3.1 \pm 1.0$ \\
\hline${ }^{25} \mathrm{Mg} \leftrightarrow{ }^{24} \mathrm{Mg}$ & $-36.7 \pm 0.8 \pm 1.0$ \\
\hline${ }^{20} \mathrm{Mg} \leftrightarrow{ }^{24} \mathrm{Mg}$ & $-29.9 \pm 0.8 \pm 1.0$ \\
\hline${ }^{20} \mathrm{Si} \leftrightarrow{ }^{28} \mathrm{Si}$ & $-5.2 \pm 6.0 \pm 0.8$ \\
\hline${ }^{30} \mathrm{Si} \leftrightarrow{ }^{28} \mathrm{Si}$ & $13.8 \pm 6.0 \pm 0.8$ \\
\hline${ }^{34} S \leftrightarrow{ }^{32} S$ & $28.9 \pm 1.5 \pm 0.8$ \\
\hline${ }^{36} S \leftrightarrow{ }^{34} S$ & $19.0 \pm 1.4 \pm 0.7$ \\
\hline${ }^{38} A r \leftrightarrow{ }^{36} A r$ & $14.9 \pm 1.0 \pm 0.7$ \\
\hline${ }^{10} \mathrm{Ar} \leftrightarrow{ }^{38} \mathrm{Ar}$ & $32.2 \pm 1.0 \pm 0.7$ \\
\hline${ }^{12} \mathrm{Ca} \leftrightarrow{ }^{10} \mathrm{Ca}$ & $39.0 \pm 0.7 \pm 0.7$ \\
\hline${ }^{13} \mathrm{Ca} \leftrightarrow{ }^{12} \mathrm{Ca}$ & $-17.8 \pm 0.7 \pm 0.7$ \\
\hline${ }^{44} \mathrm{Ca} \leftrightarrow{ }^{12} \mathrm{Ca}$ & $12.8 \pm 0.7 \pm 0.7$ \\
\hline${ }^{46} \mathrm{Ca} \leftrightarrow{ }^{44} \mathrm{Ca}$ & $-25.5 \pm 4.5 \pm 0.7$ \\
\hline${ }^{48} \mathrm{Ca} \leftrightarrow{ }^{40} \mathrm{Ca}$ & $-25.5 \pm 4.5 \pm 0.7$ \\
\hline
\end{tabular}

\begin{tabular}{|c|c|}
\hline Isotope Pair & $\Delta R_{h \alpha}^{\mu}[a m]$ \\
\hline${ }^{17} T_{i} \leftrightarrow{ }^{16} T_{i}$ & $-13.7 \pm 0.6 \pm 0.8$ \\
\hline${ }^{48} \mathrm{Ti}_{\mathrm{i}} \leftrightarrow{ }^{16} \mathrm{~T}_{\mathrm{i}}$ & $-18.4 \pm 0.7 \pm 0.8$ \\
\hline${ }^{40} \mathrm{Ti} \leftrightarrow{ }^{48} \mathrm{Ti}_{i}$ & $-23.4 \pm 0.8 \pm 0.8$ \\
\hline${ }^{{ }^{\circ}} T_{i} \leftrightarrow{ }^{48} T_{i}$ & $-27.3 \pm 0.7 \pm 0.8$ \\
\hline${ }^{52} \mathrm{Cr} \leftrightarrow{ }^{80} \mathrm{Cr}$ & $-24.9 \pm 0.5 \pm 0.8$ \\
\hline${ }^{53} \mathrm{Cr} \leftrightarrow{ }^{82} \mathrm{Cr}$ & $22.0 \pm 0.4 \pm 0.7$ \\
\hline${ }^{54} \mathrm{Cr} \leftrightarrow{ }^{52} \mathrm{Cr}$ & $58.0 \pm 0.5 \pm 0.7$ \\
\hline${ }^{56} \mathrm{Fe} \leftrightarrow{ }^{84} \mathrm{Fe}$ & $55.7 \pm 0.6 \pm 0.7$ \\
\hline${ }^{57} \mathrm{Fe} \leftrightarrow{ }^{56} \mathrm{Fe}$ & $21.0 \pm 0.7 \pm 0.7$ \\
\hline${ }^{58} \mathrm{Fe} \leftrightarrow{ }^{56} \mathrm{Fe}$ & $47.8 \pm 0.6 \pm 0.7$ \\
\hline${ }^{60} N i \leftrightarrow{ }^{58} N i$ & $47.9 \pm 0.4 \pm 0.7$ \\
\hline${ }^{11} N_{i} \leftrightarrow{ }^{60} N i$ & $14.0 \pm 0.5 \pm 0.7$ \\
\hline${ }^{62} N_{i} \leftrightarrow{ }^{60} N_{i}$ & $37.7 \pm 0.4 \pm 0.7$ \\
\hline${ }^{64} N_{i} \leftrightarrow{ }^{62} N_{i}$ & $23.9 \pm 0.4 \pm 0.7$ \\
\hline${ }^{65} \mathrm{Cu} \leftrightarrow{ }^{63} \mathrm{Cu}$ & $25.3 \pm 0.4 \pm 0.7$ \\
\hline${ }^{66} Z_{n} \leftrightarrow{ }^{84} Z_{n}$ & $26.5 \pm 0.3 \pm 0.7$ \\
\hline${ }^{88} Z n \leftrightarrow{ }^{68} Z n$ & $21.6 \pm 0.3 \pm 0.7$ \\
\hline${ }^{70} Z n \leftrightarrow{ }^{68} Z n$ & $23.2 \pm 1.3 \pm 0.7$ \\
\hline
\end{tabular}


G. FRICKE et al. Nuclear Charge Radii

TABLE V. Differences of Barrett Radii for Isotopes, $6 \leqslant Z \leqslant 82$

See page 194 for Explanation of Tables

\begin{tabular}{|c|c|}
\hline Isotope Pair & $\Delta R_{k \alpha}^{\mu}[a m]$ \\
\hline${ }^{71} G a+{ }^{69} G a$ & $18.7 \pm 0.2 \pm 0.6$ \\
\hline${ }^{72} \mathrm{Ge} \leftrightarrow{ }^{70} \mathrm{Ge}$ & $21.0 \pm 0.1 \pm 0.7$ \\
\hline${ }^{73} \mathrm{Ge} \leftrightarrow{ }^{72} \mathrm{Ge}$ & $7.9 \pm 0.2 \pm 0.7$ \\
\hline${ }^{74} \mathrm{Ge} \leftrightarrow{ }^{72} \mathrm{Ge}$ & $22.8 \pm 0.2 \pm 0.8$ \\
\hline${ }^{76} \mathrm{Ge} \leftrightarrow{ }^{71} \mathrm{Ge}$ & $9.6 \pm 0.2 \pm 0.8$ \\
\hline${ }^{77} \mathrm{Se} \leftrightarrow{ }^{70} \mathrm{Se}$ & $0.1 \pm 0.2 \pm 0.9$ \\
\hline${ }^{78} \mathrm{Se} \leftrightarrow{ }^{78} \mathrm{Se}$ & $1.9 \pm 0.2 \pm 0.9$ \\
\hline${ }^{80} \mathrm{Se} \leftrightarrow{ }^{78} \mathrm{Se}$ & $-0.5 \pm 0.1 \pm 0.8$ \\
\hline${ }^{82} \mathrm{Se} \leftrightarrow{ }^{80} \mathrm{Se}$ & $0.1 \pm 0.2 \pm 0.7$ \\
\hline${ }^{81} \mathrm{Br} \leftrightarrow{ }^{7 \theta} \mathrm{Br}$ & $-3.9 \pm 0.2 \pm 0.7$ \\
\hline${ }^{80} K r \leftrightarrow{ }^{78} K r$ & $-7.1 \pm 0.3 \pm 0.9$ \\
\hline${ }^{82} K r \leftrightarrow{ }^{80} K r$ & $-7.0 \pm 0.3 \pm 0.8$ \\
\hline${ }^{83} K r \leftrightarrow{ }^{82} K r$ & $-8.1 \pm 0.3 \pm 0.7$ \\
\hline${ }^{84} K r \leftrightarrow{ }^{82} K r$ & $-5.1 \pm 0.3 \pm 0.7$ \\
\hline${ }^{80} K r \leftrightarrow{ }^{84} K r$ & $-5.7 \pm 0.2 \pm 0.7$ \\
\hline${ }^{87} R b \leftrightarrow{ }^{85} R b$ & $-6.4 \pm 0.1 \pm 0.6$ \\
\hline${ }^{80} \mathrm{Sr} \leftrightarrow{ }^{84} \mathrm{Sr}$ & $-13.5 \pm 0.2 \pm 0.7$ \\
\hline${ }^{87} \mathrm{Sr} \leftrightarrow{ }^{86} \mathrm{Sr}$ & $-8.9 \pm 0.3 \pm 0.6$ \\
\hline${ }^{88} \mathrm{Sr} \leftrightarrow{ }^{86} \mathrm{Sr}$ & $-9.2 \pm 0.1 \pm 0.6$ \\
\hline
\end{tabular}

\begin{tabular}{|c|c|}
\hline Isotope Pair & $\Delta R_{k \alpha}^{\mu}[a m]$ \\
\hline${ }^{81} \mathrm{Zr} \leftrightarrow{ }^{90} \mathrm{Zr}$ & $19.1 \pm 0.2 \pm 0.5$ \\
\hline${ }^{92} \mathrm{Zr} \leftrightarrow{ }^{80} \mathrm{Zr}$ & $45.5 \pm 0.1 \pm 0.5$ \\
\hline${ }^{94} Z r \leftrightarrow{ }^{92} Z r$ & $32.4 \pm 0.1 \pm 0.5$ \\
\hline${ }^{96} Z r \leftrightarrow{ }^{94} Z r$ & $23.9 \pm 0.2 \pm 0.5$ \\
\hline${ }^{94} \mathrm{Mo} \leftrightarrow{ }^{92} \mathrm{Mo}$ & $45.7 \pm 0.1 \pm 0.6$ \\
\hline${ }^{95} \mathrm{Mo} \nleftarrow{ }^{94} \mathrm{Mo}$ & $12.8 \pm 0.1 \pm 0.6$ \\
\hline${ }^{96} \mathrm{Mo} \leftrightarrow{ }^{94} \mathrm{Mo}$ & $40.2 \pm 0.1 \pm 0.6$ \\
\hline${ }^{97} \mathrm{Mo} \leftrightarrow{ }^{90} \mathrm{Mo}$ & $4.1 \pm 0.1 \pm 0.6$ \\
\hline${ }^{98} \mathrm{Mo} \leftrightarrow{ }^{96} \mathrm{Mo}$ & $30.8 \pm 0.1 \pm 0.6$ \\
\hline${ }^{100} M o \leftrightarrow{ }^{98} M o$ & $47.1 \pm 0.1 \pm 0.7$ \\
\hline${ }^{98} R u \leftrightarrow{ }^{80} R u$ & $39.4 \pm 1.0 \pm 0.6$ \\
\hline${ }^{98} R u \leftrightarrow{ }^{98} R u$ & $15.0 \pm 1.0 \pm 0.6$ \\
\hline${ }^{100} R u \leftrightarrow{ }^{98} R u$ & $39.0 \pm 1.0 \pm 0.6$ \\
\hline${ }^{101} R u \leftrightarrow{ }^{100} R u$ & $10.4 \pm 0.5 \pm 0.6$ \\
\hline${ }^{102} R u \leftrightarrow{ }^{100} R u$ & $35.8 \pm 0.3 \pm 0.7$ \\
\hline${ }^{104} R u \leftrightarrow{ }^{102} R u$ & $36.7 \pm 0.2 \pm 0.7$ \\
\hline${ }^{104} P d \leftrightarrow{ }^{102} P d$ & $32.0 \pm 0.6 \pm 0.6$ \\
\hline${ }^{105} P d \leftrightarrow{ }^{104} P d$ & $9.4 \pm 0.8 \pm 0.7$ \\
\hline
\end{tabular}


TABLE V. Differences of Barrett Radii for Isotopes, $6 \leqslant Z \leqslant 82$ See page 194 for Explanation of Tables

\begin{tabular}{|c|c|c|}
\hline Isotope & Pair & $\Delta R_{k \alpha}^{\mu}[a m]$ \\
\hline${ }^{106} \mathrm{Pd} \leftrightarrow$ & ${ }^{104} P d$ & $30.5 \pm 0.5 \pm 0.6$ \\
\hline${ }^{108} \mathrm{Pd} \leftrightarrow$ & ${ }^{100} \mathrm{Pd}$ & $30.7 \pm 0.3 \pm 0.7$ \\
\hline${ }^{110} \mathrm{Pd} \leftrightarrow$ & ${ }^{108} P d$ & $27.7 \pm 0.4 \pm 0.7$ \\
\hline${ }^{109} \mathrm{Ag} \leftrightarrow$ & ${ }^{107} \mathrm{Ag}$ & $27.2 \pm 0.1 \pm 0.6$ \\
\hline${ }^{108} \mathrm{Cd} \leftrightarrow$ & ${ }^{106} \mathrm{Cd}$ & $25.3 \pm 0.3 \pm 0.6$ \\
\hline${ }^{110} \mathrm{Cd} \leftrightarrow$ & ${ }^{108} \mathrm{Cd}$ & $25.5 \pm 0.2 \pm 0.6$ \\
\hline${ }^{111} C d \leftrightarrow$ & ${ }^{110} \mathrm{Cd}$ & $6.4 \pm 0.2 \pm 0.6$ \\
\hline${ }^{112} C d \leftrightarrow$ & ${ }^{110} \mathrm{Cd}$ & $24.7 \pm 0.2 \pm 0.6$ \\
\hline${ }^{113} \mathrm{Cd} \leftrightarrow$ & ${ }^{112} C d$ & $6.2 \pm 0.1 \pm 0.6$ \\
\hline${ }^{114} C d \leftrightarrow$ & ${ }^{112} C d$ & $22.5 \pm 0.1 \pm 0.6$ \\
\hline${ }^{116} C d \leftrightarrow$ & ${ }^{114} \mathrm{Cd}$ & $19.1 \pm 0.1 \pm 0.6$ \\
\hline${ }^{118} I n \leftrightarrow$ & ${ }^{113} \operatorname{In}$ & $20.9 \pm 1.2 \pm 0.7$ \\
\hline${ }^{114} S n \leftrightarrow$ & ${ }^{112} S n$ & $20.9 \pm 0.3 \pm 0.5$ \\
\hline${ }^{116} S n \leftrightarrow$ & ${ }^{114} S n$ & $20.9 \pm 0.3 \pm 0.5$ \\
\hline${ }^{117} S n \leftrightarrow$ & ${ }^{116} S n$ & $6.2 \pm 0.3 \pm 0.5$ \\
\hline${ }^{118} \mathrm{Sn} \leftrightarrow$ & ${ }^{116} \mathrm{Sn}$ & $19.8 \pm 0.4 \pm 0.5$ \\
\hline${ }^{119} S n \leftrightarrow$ & ${ }^{118} S n$ & $3.7 \pm 0.4 \pm 0.5$ \\
\hline${ }^{120} \mathrm{Sn} \leftrightarrow$ & ${ }^{118} \mathrm{Sn}$ & $18.0 \pm 0.4 \pm 0.6$ \\
\hline${ }^{122} \mathrm{Sn} \leftrightarrow$ & ${ }^{120} S n$ & $15.7 \pm 0.5 \pm 0.6$ \\
\hline${ }^{124} S n \leftrightarrow$ & ${ }^{122} S n$ & $13.4 \pm 0.5 \pm 0.5$ \\
\hline
\end{tabular}

\begin{tabular}{|c|c|c|}
\hline Isotope I & Pair & $\Delta R_{k a}^{\mu}[a m]$ \\
\hline${ }^{123} \mathrm{Sb} \leftrightarrow$ & ${ }^{121} S b$ & $10.5 \pm 0.2 \pm 0.6$ \\
\hline${ }^{123} \mathrm{Te} \leftrightarrow$ & ${ }^{122} \mathrm{Te}$ & $3.3 \pm 1.1 \pm 0.6$ \\
\hline${ }^{124} \mathrm{Te} \leftrightarrow$ & ${ }^{122} \mathrm{Te}$ & $12.2 \pm 1.1 \pm 0.6$ \\
\hline${ }^{125} \mathrm{Te} \leftrightarrow$ & ${ }^{124} \mathrm{Te}$ & $2.2 \pm 0.1 \pm 0.5$ \\
\hline${ }^{126} \mathrm{Te} \leftrightarrow$ & ${ }^{124} \mathrm{Te}$ & $10.5 \pm 0.1 \pm 0.5$ \\
\hline${ }^{128} \mathrm{Te} \leftrightarrow$ & ${ }^{120} \mathrm{Te}$ & $10.1 \pm 0.1 \pm 0.5$ \\
\hline${ }^{130} \mathrm{Te} \leftrightarrow$ & ${ }^{128} \mathrm{Te}$ & $9.5 \pm 0.1 \pm 0.5$ \\
\hline${ }^{126} \mathrm{Xe} \leftrightarrow$ & ${ }^{124} X e$ & $10.5 \pm 0.3 \pm 0.4$ \\
\hline${ }^{128} \mathrm{Xe} \leftrightarrow$ & ${ }^{126} X e$ & $6.8 \pm 0.4 \pm 0.5$ \\
\hline${ }^{120} \mathrm{Xe} \leftrightarrow$ & ${ }^{128} X e$ & $1.0 \pm 0.3 \pm 0.5$ \\
\hline${ }^{130} \mathrm{Xe} \leftrightarrow$ & ${ }^{128} \mathrm{Xe}$ & $10.0 \pm 0.6 \pm 0.5$ \\
\hline${ }^{131} \mathrm{Xe} \leftrightarrow$ & ${ }^{130} X e$ & $-2.6 \pm 0.6 \pm 0.5$ \\
\hline${ }^{132} \mathrm{Xe} \leftrightarrow$ & ${ }^{150} X e$ & $4.4 \pm 0.6 \pm 0.5$ \\
\hline${ }^{134} \mathrm{Xe} \leftrightarrow$ & ${ }^{132} X e$ & $7.2 \pm 0.1 \pm 0.5$ \\
\hline${ }^{136} \mathrm{Xe} \leftrightarrow$ & ${ }^{134} X e$ & $9.4 \pm 0.1 \pm 0.5$ \\
\hline${ }^{135} B a \leftrightarrow$ & ${ }^{134} B a$ & $-2.9 \pm 0.1 \pm 0.5$ \\
\hline${ }^{198} \mathrm{Ba} \leftrightarrow$ & ${ }^{134} B a$ & $4.3 \pm 0.1 \pm 0.5$ \\
\hline${ }^{137} \mathrm{Ba} \leftrightarrow$ & ${ }^{136} B a$ & $-0.7 \pm 0.2 \pm 0.5$ \\
\hline${ }^{138} B a \leftrightarrow$ & ${ }^{136} B a$ & $8.1 \pm 0.1 \pm 0.5$ \\
\hline
\end{tabular}


G. FRICKE et al. Nuclear Charge Radii

TABLE V. Differences of Barrett Radii for Isotopes, $6 \leqslant Z \leqslant 82$

See page 194 for Explanation of Tables

\begin{tabular}{|c|c|c|}
\hline Isotope & Pair & $\Delta R_{h_{\alpha}}^{\mu_{\alpha}}[a m]$ \\
\hline${ }^{142} \mathrm{Ce} \leftrightarrow$ & ${ }^{140} \mathrm{Ce}$ & $38.2 \pm 0.2 \pm 0.5$ \\
\hline${ }^{143} N d \leftrightarrow$ & ${ }^{142} N d$ & $13.9 \pm 0.3 \pm 0.5$ \\
\hline${ }^{144} N d \leftrightarrow$ & ${ }^{142} N d$ & $35.5 \pm 0.1 \pm 0.5$ \\
\hline${ }^{148} N d \leftrightarrow$ & ${ }^{144} N d$ & $15.4 \pm 0.3 \pm 0.6$ \\
\hline${ }^{146} \mathrm{Nd} H$ & ${ }^{144} N d$ & $34.9 \pm 0.1 \pm 0.5$ \\
\hline${ }^{148} N d \leftrightarrow$ & ${ }^{140} N d$ & $38.4 \pm 0.2 \pm 0.4$ \\
\hline${ }^{148} \mathrm{Sm} \leftrightarrow$ & ${ }^{147} \mathrm{Sm}$ & $19.9 \pm 0.1 \pm 0.4$ \\
\hline${ }^{140} \mathrm{Sm} \leftrightarrow$ & ${ }^{148} \mathrm{Sm}$ & $12.9 \pm 0.1 \pm 0.4$ \\
\hline${ }^{162} \mathrm{Sm} \leftrightarrow$ & ${ }^{180} \mathrm{Sm}$ & $55.2 \pm 0.8^{\star}$ \\
\hline${ }^{154} \mathrm{Sm} \leftrightarrow$ & ${ }^{162} \mathrm{Sm}$ & $31.3 \pm 1.7^{\star}$ \\
\hline${ }^{169} E u \leftrightarrow$ & ${ }^{151} E_{u}$ & $72.9 \pm 0.1 \pm 0.4$ \\
\hline${ }^{185} \mathrm{Gd} \leftrightarrow$ & ${ }^{154} G d$ & $15.3 \pm 0.7 \pm 0.7$ \\
\hline${ }^{150} \mathrm{Gd} \leftrightarrow$ & ${ }^{164} G d$ & $28.5 \pm 0.7 \pm 0.8$ \\
\hline${ }^{187} \mathrm{Gd} \leftrightarrow$ & ${ }^{156} G d$ & $4.7 \pm 0.3 \pm 0.8$ \\
\hline${ }^{158} G d \leftrightarrow$ & ${ }^{150} \mathrm{Gd}$ & $20.0 \pm 0.1 \pm 0.8$ \\
\hline${ }^{160} G d \leftrightarrow$ & ${ }^{158} \mathrm{Gd}$ & $21.2 \pm 0.2 \pm 0.8$ \\
\hline${ }^{162} D y \leftrightarrow$ & ${ }^{161} D y$ & $14.3 \pm 0.4 \pm 0.8$ \\
\hline${ }^{163} D y \leftrightarrow$ & ${ }^{162} D y$ & $5.9 \pm 0.2 \pm 0.8$ \\
\hline${ }^{104} D y \leftrightarrow$ & ${ }^{162} D y$ & $19.4 \pm 0.2 \pm 0.8$ \\
\hline
\end{tabular}

* Total error including uncertainties in calculating nuclear polarization corrections and experimental errors is given.

\begin{tabular}{|c|c|c|}
\hline Isotope & Pair & $\Delta R_{k \alpha}^{\mu}[a m]$ \\
\hline${ }^{167} \mathrm{Er} \leftrightarrow$ & ${ }^{106} \mathrm{Er}$ & $7.5 \pm 0.3 \pm 0.6$ \\
\hline${ }^{108} \mathrm{Er} \leftrightarrow$ & ${ }^{100} \mathrm{Er}$ & $18.4 \pm 0.3 \pm 0.6$ \\
\hline${ }^{170} E r \leftrightarrow$ & ${ }^{108} \mathrm{Er}$ & $19.9 \pm 0.3 \pm 0.6$ \\
\hline${ }^{172} Y b \leftrightarrow$ & ${ }^{170} Y b$ & $18.0 \pm 0.6^{\dagger}$ \\
\hline${ }^{172} Y b \leftrightarrow$ & ${ }^{171} Y b$ & $9.8 \pm 0.6^{\dagger}$ \\
\hline${ }^{174} Y b \leftrightarrow$ & ${ }^{172} Y b$ & $14.8 \pm 0.4^{\dagger}$ \\
\hline${ }^{174} Y b \leftrightarrow$ & ${ }^{173} Y b$ & $8.6 \pm 0.7^{\dagger}$ \\
\hline${ }^{176} Y b \leftrightarrow$ & ${ }^{174} Y b$ & $13.5 \pm 0.5^{\dagger}$ \\
\hline${ }^{177} H f \leftrightarrow$ & ${ }^{17 \theta} H f$ & $3.9 \pm 0.4 \pm 0.6$ \\
\hline${ }^{178} \mathrm{Hf} \leftrightarrow$ & ${ }^{17 \theta} H f$ & $11.6 \pm 0.4 \pm 0.6$ \\
\hline${ }^{179} H f \leftrightarrow$ & ${ }^{178} H f$ & $5.5 \pm 0.5 \pm 0.6$ \\
\hline${ }^{180} H f \leftrightarrow$ & ${ }^{178} \mathrm{Hf}$ & $12.7 \pm 0.5 \pm 0.6$ \\
\hline${ }^{184} W \leftrightarrow$ & ${ }^{182} W$ & $13.5 \pm 0.3 \pm 0.6$ \\
\hline${ }^{186} W \leftrightarrow$ & ${ }^{184} W$ & $12.1 \pm 0.3 \pm 0.6$ \\
\hline${ }^{188} \mathrm{Os} \leftrightarrow$ & ${ }^{186} \mathrm{Os}$ & $12.3 \pm 0.5 \pm 0.6$ \\
\hline${ }^{190} \mathrm{Os} \leftrightarrow$ & ${ }^{188} \mathrm{Os}$ & $10.6 \pm 0.4 \pm 0.6$ \\
\hline${ }^{102} \mathrm{Os} \leftrightarrow$ & ${ }^{190} \mathrm{Os}$ & $10.0 \pm 0.1 \pm 0.6$ \\
\hline
\end{tabular}

† The shifts of the 1s state - taken from [Ze75] - are given. The quoted error includes only experimental uncertainties. 
G. FRICKE et al. Nuclear Charge Radii

TABLE V. Differences of Barrett Radii for Isotopes, $6 \leqslant Z \leqslant 82$ See page 194 for Explanation of Tables

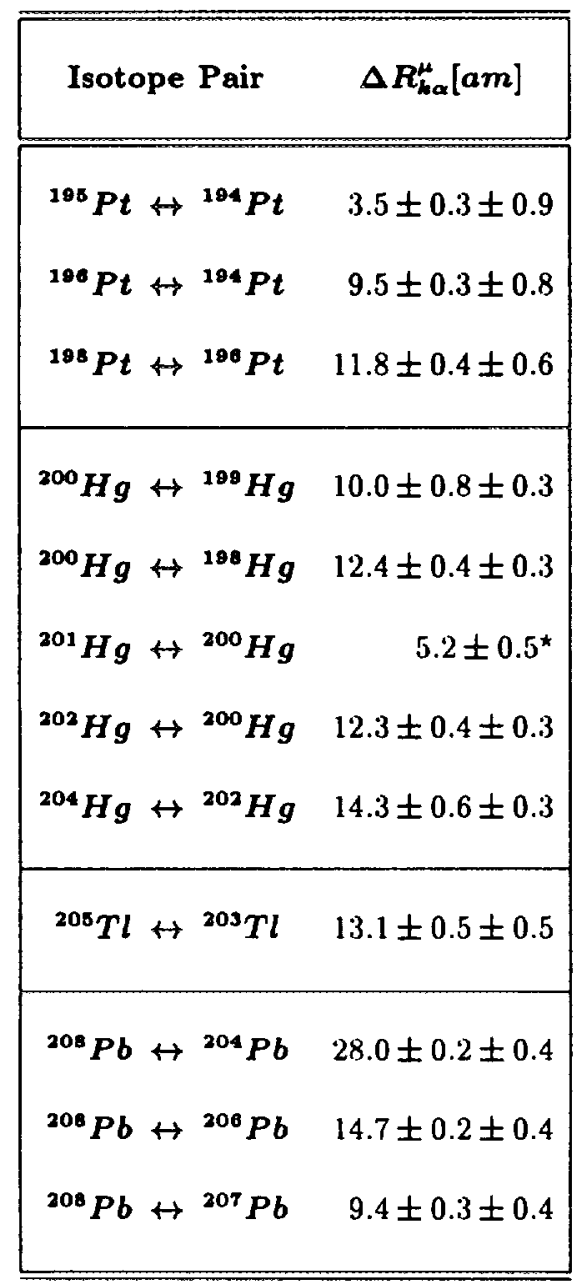

* Only the experimental error is given 
G. FRICKE et al. Nuclear Charge Radii

TABLE VI. Confit Matrices for Muonic $2 p \rightarrow 1 s$ Energy Differences of Isotones, $10 \leqslant Z \leqslant 82$ See page 194 for Explanation of Tables

\begin{tabular}{|c|rrrrr|}
\hline$[\mathrm{keV}]$ & ${ }^{19} \mathrm{~F}$ & ${ }^{20} \mathrm{Ne}$ & ${ }^{22} \mathrm{Ne}$ & ${ }^{23} \mathrm{Na}$ & ${ }^{24} \mathrm{Mg}$ \\
\hline${ }^{19} \mathrm{~F}$ & & -38.767 & -38.998 & -81.714 & -128.020 \\
& 5 & 5 & 2 & 3 \\
${ }^{20} \mathrm{Ne}$ & & -0.230 & -42.947 & -89.252 \\
& & 6 & 5 & 5 \\
${ }^{22} \mathrm{Ne}$ & & & -42.717 & -89.022 \\
& & & 4 & 4 \\
${ }^{23} \mathrm{Na}$ & & & & & \\
& & & & & \\
\hline \hline
\end{tabular}

\begin{tabular}{|c|c|c|c|c|c|c|}
\hline$[\mathrm{keV}]$ & ${ }^{20} \mathrm{Mg}$ & ${ }^{27} A l$ & ${ }^{28} \mathrm{Si}$ & ${ }^{31} P$ & ${ }^{32} S$ & ${ }^{34} S$ \\
\hline \multirow[t]{2}{*}{${ }^{26} \mathrm{Mg}$} & & -50.083 & -103.428 & -160.055 & -219.585 & -219.361 \\
\hline & & 3 & 6 & 12 & 12 & 15 \\
\hline \multirow[t]{2}{*}{${ }^{27} A l$} & & & -53.345 & -109.972 & -169.502 & -169.278 \\
\hline & & & 5 & 11 & 12 & 15 \\
\hline \multirow[t]{2}{*}{${ }^{20} \mathrm{Si}$} & & & & -56.627 & -116.158 & -115.933 \\
\hline & & & & 13 & 13 & 16 \\
\hline \multirow[t]{2}{*}{${ }^{31} P$} & & & & & -59.530 & -59.306 \\
\hline & & & & & 14 & 16 \\
\hline \multirow[t]{2}{*}{${ }^{32} S$} & & & & & & -0.224 \\
\hline & & & & & & 16 \\
\hline${ }^{34} S$ & & & & & & \\
\hline
\end{tabular}


TABLE VI. Confit Matrices for Muonic $2 p \rightarrow 1 s$ Energy Differences of Isotones, $10 \leqslant Z \leqslant 82$ See page 194 for Explanation of Tables

\begin{tabular}{|c|c|c|c|c|c|c|c|c|}
\hline [keV] & ${ }^{6 B} Z n$ & ${ }^{69} \mathrm{Ga}$ & ${ }^{70} \mathrm{Ge}$ & ${ }^{74} \mathrm{Ge}$ & ${ }^{75} \mathrm{As}$ & ${ }^{76} \mathrm{Se}$ & ${ }^{78} \mathrm{Se}$ & ${ }^{79} \mathrm{Br}$ \\
\hline \multirow[t]{2}{*}{${ }^{68} Z n$} & & -89.254 & -178.338 & -174.208 & -265.603 & -355.600 & -355.331 & -448.225 \\
\hline & & 40 & 38 & 45 & 40 & 42 & 41 & 42 \\
\hline \multirow[t]{2}{*}{${ }^{60} \mathrm{Ga}$} & 90.236 & & -89.084 & -84.954 & -176.349 & -266.346 & -266.077 & -358.971 \\
\hline & 33 & & 26 & 28 & 18 & 24 & 21 & 24 \\
\hline \multirow[t]{2}{*}{${ }^{70} \mathrm{Ge}$} & 180.273 & 90.037 & & 4.130 & -87.265 & -177.262 & -176.994 & -269.888 \\
\hline & 29 & 19 & & $3: 3$ & 26 & 30 & 28 & 30 \\
\hline \multirow[t]{2}{*}{${ }^{74} \mathrm{Ge}$} & 176.081 & 85.845 & -4.192 & & -91.395 & -181.392 & -181.123 & -274.017 \\
\hline & 35 & 20 & 21 & & 29 & 33 & 30 & 32 \\
\hline \multirow[t]{2}{*}{${ }^{75} A s$} & 268.864 & 178.628 & 88.591 & 92.783 & & -89.997 & -89.728 & -182.622 \\
\hline & 33 & 16 & 20 & 20 & & 24 & 21 & 24 \\
\hline \multirow[t]{2}{*}{${ }^{76} \mathrm{Se}$} & 359.820 & 269.584 & 179.547 & 183.739 & 90.956 & & 0.269 & -92.625 \\
\hline & 35 & 19 & 22 & 23 & 19 & & 25 & 28 \\
\hline \multirow[t]{2}{*}{${ }^{78} \mathrm{Se}$} & 359.566 & 269.3330 & 179.293 & 183.485 & 90.702 & -0.254 & & -92.894 \\
\hline & 34 & 17 & 20 & 21 & 17 & 18 & & 25 \\
\hline \multirow[t]{2}{*}{${ }^{79} \mathrm{Br}$} & 453.998 & 363.762 & 273.725 & 277.917 & 185.134 & 94.178 & 94.432 & \\
\hline & 36 & 20 & $2 ; 3$ & 24 & 20 & $2: 3$ & 20 & \\
\hline
\end{tabular}

\begin{tabular}{|c|c|c|c|c|c|c|c|c|}
\hline$[\mathbf{k e V}]$ & ${ }^{80} \mathrm{Se}$ & ${ }^{81} B r$ & ${ }^{22} K r$ & ${ }^{86} K r$ & ${ }^{87} R b$ & ${ }^{88} S r$ & ${ }^{89} Y$ & ${ }^{90} Z r$ \\
\hline \multirow[t]{2}{*}{${ }^{\circ 0} \mathrm{Se}$} & & -93.354 & -185.837 & -187.3332 & -283.170 & -377.814 & -4733.934 & -568.509 \\
\hline & & 28 & 46 & 38 & 24 & 17 & 18 & 19 \\
\hline \multirow{2}{*}{${ }^{81} \mathrm{Br}$} & 94.857 & & -92.482 & $-9: 3.977$ & -189.816 & -284.459 & -380.580 & -475.154 \\
\hline & 23 & & 42 & 31 & 26 & 26 & 21 & 27 \\
\hline \multirow[t]{2}{*}{${ }^{82} K r$} & 188.572 & 93.715 & & -1.495 & -97.3334 & -191.977 & -288.097 & -382.672 \\
\hline & 42 & 44 & & 39 & 48 & 44 & 45 & 45 \\
\hline \multirow[t]{2}{*}{${ }^{86} \mathrm{Kr}$} & 190.094 & 95.237 & 1.522 & & -95.839 & -190.482 & -286.602 & -381.177 \\
\hline & 36 & 40 & 33 & & 29 & 36 & 37 & 37 \\
\hline \multirow[t]{2}{*}{${ }^{87} R b$} & 287.500 & 192.643 & 98.928 & 97.406 & & -94.644 & -190.764 & -285.338 \\
\hline & 18 & 24 & 42 & 37 & & 22 & 22 & $2: 3$ \\
\hline \multirow[t]{2}{*}{${ }^{88} S r$} & $38: 3.788$ & 288.931 & 195.216 & $19: 3.694$ & 96.288 & & -96.120 & -190.695 \\
\hline & 14 & 21 & 41 & 35 & 15 & & 11 & 13 \\
\hline \multirow[t]{2}{*}{${ }^{8 \theta} Y$} & 481.658 & 386.801 & 293.086 & 291.564 & 194.158 & 97.870 & & -94.575 \\
\hline & 15 & 22 & 41 & 35 & 16 & 8 & & 13 \\
\hline \multirow[t]{2}{*}{${ }^{\circ 0} Z r$} & 578.096 & 483.239 & 389.524 & 388.002 & 290.596 & 194.308 & 96.438 & \\
\hline & 15 & 22 & 41 & 36 & 16 & 10 & 10 & \\
\hline
\end{tabular}


G. FRICKE et al. Nuclear Charge Radii

TABLE VI. Confit Matrices for Muonic $2 p \rightarrow 1 s$ Energy Differences of Isotones, $10 \leqslant Z \leqslant 82$ See page 194 for Explanation of Tables

\begin{tabular}{|c|c|c|c|c|c|c|c|}
\hline$[\mathrm{keV}]$ & ${ }^{82} Z r$ & ${ }^{83} \mathrm{Nb}$ & ${ }^{94} \mathrm{Mo}$ & ${ }^{100} M O$ & ${ }^{102} R u$ & ${ }^{103} R h$ & ${ }^{104} P d$ \\
\hline \multirow[t]{2}{*}{${ }^{92} Z r$} & & -96.191 & -190.438 & -166.795 & -357.177 & -454.067 & -550.377 \\
\hline & & 26 & 28 & 30 & 39 & 35 & 140 \\
\hline \multirow[t]{2}{*}{${ }^{93} \mathrm{Nb}$} & 98.412 & & -94.247 & -70.605 & -260.986 & -357.876 & -454.186 \\
\hline & 19 & & 29 & 31 & 40 & 36 & 141 \\
\hline \multirow[t]{2}{*}{${ }^{84} \mathrm{Mo}$} & 194.472 & 96.060 & & 23.642 & -166.740 & -263.629 & -359.939 \\
\hline & 19 & 21 & & 33 & 42 & 38 & 141 \\
\hline \multirow[t]{2}{*}{${ }^{100} \mathrm{Mo}$} & 170.687 & 72.275 & -23.784 & & -190.382 & -287.272 & -383.582 \\
\hline & 20 & 22 & 22 & & 43 & 40 & 142 \\
\hline \multirow[t]{2}{*}{${ }^{102} R u$} & 365.639 & 267.227 & 171.167 & 194.952 & & -90.890 & -193.200 \\
\hline & 31 & 33 & 33 & 34 & & 46 & 143 \\
\hline \multirow[t]{2}{*}{${ }^{103} R h$} & 464.878 & 366.466 & 270.407 & 294.191 & 99.240 & & -96.310 \\
\hline & 26 & 27 & 28 & 28 & 35 & & 142 \\
\hline \multirow[t]{2}{*}{${ }^{104} \mathrm{Pd}$} & 563.884 & 465.472 & 369.412 & 393.197 & 198.245 & 99.006 & \\
\hline & 121 & 121 & 121 & 122 & 123 & 122 & \\
\hline
\end{tabular}

\begin{tabular}{|c|c|c|c|c|c|c|c|}
\hline [keV] & ${ }^{108} \mathrm{Pd}$ & ${ }^{108} \mathrm{Ag}$ & ${ }^{110} C d$ & ${ }^{114} C d$ & ${ }^{115} I n$ & ${ }^{123} S b$ & ${ }^{124} \mathrm{Te}$ \\
\hline \multirow[t]{2}{*}{${ }^{108} P d$} & & -97.510 & -195.198 & -182.301 & -280.452 & -452.010 & -5339.937 \\
\hline & & 78 & 85 & 77 & 82 & 106 & 77 \\
\hline \multirow[t]{2}{*}{${ }^{100} \mathrm{Ag}$} & 100.278 & & -97.688 & -84.791 & -182.942 & -354.500 & -442.427 \\
\hline & 52 & & 56 & $4: 3$ & 47 & 84 & 40 \\
\hline \multirow[t]{2}{*}{${ }^{110} \mathrm{Cd}$} & 200.832 & 100.554 & & 12.897 & -85.255 & -256.813 & -344.740 \\
\hline & 61 & 46 & & 55 & 61 & 91 & 54 \\
\hline \multirow[t]{2}{*}{${ }^{114} \mathrm{Cd}$} & 187.853 & 87.575 & -12.979 & & -98.152 & -269.709 & -357.637 \\
\hline & 52 & 33 & 46 & & 49 & 84 & 39 \\
\hline \multirow[t]{2}{*}{${ }^{115} I n$} & 289.766 & 189.489 & 88.934 & 101.913 & & -171.558 & -259.485 \\
\hline & 58 & 37 & 52 & 41 & & 87 & 47 \\
\hline \multirow[t]{2}{*}{${ }^{123} S b$} & 467.400 & 367.122 & 266.568 & 279.547 & 177.634 & & -87.927 \\
\hline & 73 & 60 & 69 & 61 & 65 & & 82 \\
\hline \multirow[t]{2}{*}{${ }^{124} \mathrm{Te}$} & 557.597 & 457.319 & 356.765 & 369.744 & 267.831 & 90.197 & \\
\hline & 52 & 32 & 46 & 32 & 41 & 60 & \\
\hline
\end{tabular}


TABLE VI. Confit Matrices for Muonic $2 p \rightarrow 1 s$ Energy Differences of Isotones, $10 \leqslant Z \leqslant 82$ See page 194 for Explanation of Tables

\begin{tabular}{|c|c|c|c|c|c|c|}
\hline$[\mathrm{keV}]$ & ${ }^{126} \mathrm{Te}$ & ${ }^{127} I$ & ${ }^{128} X e$ & ${ }^{132} X e$ & ${ }^{139} \mathrm{Cs}$ & ${ }^{134} B a$ \\
\hline \multirow[t]{2}{*}{${ }^{120} \mathrm{Te}$} & & -88.364 & -176.589 & -171.740 & -261.706 & -347.789 \\
\hline & & 43 & 105 & 65 & 46 & 40 \\
\hline \multirow[t]{2}{*}{${ }^{127} I$} & 92.864 & & -88.225 & -83.376 & -173.342 & -259.424 \\
\hline & 39 & & 108 & 71 & 47 & 47 \\
\hline \multirow[t]{2}{*}{${ }^{128} X e$} & 183.456 & 90.592 & & 4.849 & -85.117 & -171.199 \\
\hline & 104 & 107 & & 118 & 109 & 107 \\
\hline \multirow[t]{2}{*}{${ }^{132} X e$} & 178.408 & 85.544 & -5.048 & & -89.966 & -176.048 \\
\hline & 45 & 52 & 109 & & 72 & 69 \\
\hline \multirow[t]{2}{*}{${ }^{133} \mathrm{Cs}$} & 271.757 & 178.894 & 88.302 & 93.350 & & -86.083 \\
\hline & 37 & 36 & 106 & 50 & & 50 \\
\hline \multirow[t]{2}{*}{${ }^{134} B a$} & 361.805 & 268.942 & 178.349 & 183.398 & 90.048 & \\
\hline & 31 & 40 & 105 & 48 & 38 & \\
\hline
\end{tabular}

\begin{tabular}{|c|c|c|c|c|}
\hline$[\mathrm{keV}]$ & ${ }^{138} \mathrm{Ba}$ & ${ }^{139} L a$ & ${ }^{140} \mathrm{Ce}$ & ${ }^{141} \mathrm{Pr}$ \\
\hline \multirow[t]{2}{*}{${ }^{138} B a$} & & -89.368 & -175.200 & -263.719 \\
\hline & & 39 & 35 & 27 \\
\hline \multirow[t]{2}{*}{${ }^{139} L a$} & 93.420 & & -85.832 & -174.352 \\
\hline & 33 & & 43 & 45 \\
\hline \multirow[t]{2}{*}{${ }^{140} \mathrm{Ce}$} & 182.946 & 89.526 & & -88.520 \\
\hline & 32 & 32 & & 41 \\
\hline \multirow[t]{2}{*}{${ }^{141} P r$} & 275.607 & 182.187 & 92.661 & \\
\hline & 25 & 40 & 39 & \\
\hline
\end{tabular}


TABLE VII. Differences of Barrett Radii for Isotones, $8 \leqslant Z \leqslant 126$ See page 194 for Explanation of Tables

\begin{tabular}{|c|c|c|c|}
\hline \multicolumn{3}{|c|}{ Isotone Pair } & $\Delta R_{k \alpha}^{\mu}[a m]$ \\
\hline${ }^{10} \mathrm{O}$ & $H$ & ${ }^{14} C^{\star}$ & $242.1 \pm 12.6 \pm 1.1$ \\
\hline${ }^{20} \mathrm{Ne}$ & $H$ & ${ }^{19} \mathrm{~F}$ & $136.5 \pm 3.2 \pm 1.0$ \\
\hline${ }^{20} \mathrm{Ne}$ & $\leftrightarrow$ & ${ }^{18} \mathrm{O}$ & $297.6 \pm 14.0 \pm 1.0$ \\
\hline${ }^{23} \mathrm{Na}$ & $\leftrightarrow$ & ${ }^{22} \mathrm{Ne}$ & $50.6 \pm 1.8 \pm 0.9$ \\
\hline${ }^{24} M g$ & $\leftrightarrow$ & ${ }^{22} \mathrm{Ne}$ & $130.5 \pm 1.6 \pm 1.0$ \\
\hline${ }^{27} \mathrm{Al}$ & $\leftrightarrow$ & ${ }^{30} \mathrm{Mg}$ & $36.2 \pm 0.7 \pm 1.1$ \\
\hline${ }^{20} \mathrm{Si}$ & $\leftrightarrow$ & ${ }^{20} \mathrm{Mg}$ & $112.0 \pm 1.2 \pm 1.4$ \\
\hline${ }^{32} S$ & $\leftrightarrow$ & ${ }^{31} P$ & $92.3 \pm 1.5 \pm 1.0$ \\
\hline${ }^{32} S$ & $\leftrightarrow$ & ${ }^{30} S i^{\star}$ & $164.2 \pm 6.7 \pm 0.8$ \\
\hline${ }^{36} \mathrm{Ar}$ & $\leftrightarrow$ & ${ }^{34} S$ & $133.4 \pm 1.0 \pm 1.1$ \\
\hline${ }^{40} \mathrm{Ca}$ & $\leftrightarrow$ & ${ }^{39} \mathrm{~K}$ & $55.1 \pm 2.0 \pm 0.7$ \\
\hline${ }^{42} \mathrm{Ca}$ & $\leftrightarrow$ & ${ }^{ \pm 0} A r^{\star}$ & $103.2 \pm 1.9 \pm 1.0$ \\
\hline${ }^{45} S c$ & $\leftrightarrow$ & ${ }^{44} \mathrm{Ca}$ & $35.3 \pm 1.3 \pm 0.9$ \\
\hline${ }^{46} \mathrm{Ti}$ & $\leftrightarrow$ & ${ }^{14} \mathrm{Ca}$ & $111.7 \pm 1.3 \pm 0.8$ \\
\hline${ }^{51} V$ & $\leftrightarrow$ & ${ }^{80} \mathrm{Ti}$ & $36.0 \pm 1.0 \pm 0.7$ \\
\hline${ }^{52} \mathrm{Cr}$ & $\leftrightarrow$ & ${ }^{80} \mathrm{Ti}$ & $89.1 \pm 0.8 \pm 0.7$ \\
\hline${ }^{\mathrm{BE}} M n$ & $\leftrightarrow$ & ${ }^{54} \mathrm{Cr}$ & $24.8 \pm 0.6 \pm 0.8$ \\
\hline${ }^{56} \mathrm{Fe}$ & $\leftrightarrow$ & ${ }^{54} \mathrm{Cr}$ & $63.8 \pm 0.7 \pm 0.9$ \\
\hline${ }^{59} \mathrm{Co}$ & $\leftrightarrow$ & ${ }^{58} \mathrm{Fe}$ & $16.4 \pm 0.8 \pm 0.8$ \\
\hline${ }^{60} \mathrm{Ni}$ & $\leftrightarrow$ & ${ }^{58} \mathrm{Fe}$ & $47.2 \pm 0.8 \pm 0.7$ \\
\hline${ }^{\mathrm{es}} \mathrm{Cu}$ & $\leftrightarrow$ & ${ }^{62} \mathrm{Ni}$ & $52.0 \pm 0.4 \pm 0.8$ \\
\hline
\end{tabular}

\begin{tabular}{|c|c|c|c|}
\hline \multicolumn{3}{|c|}{ Isotone Pair } & $\Delta R_{k \alpha}^{k}[a m]$ \\
\hline${ }^{04} Z n$ & $\leftrightarrow$ & ${ }^{62} \mathrm{Ni}$ & $109.2 \pm 0.4 \pm 0.7$ \\
\hline${ }^{69} \mathrm{Ga}$ & $\leftrightarrow$ & ${ }^{\circ B} Z_{n}$ & $40.0 \pm 0.4 \pm 0.7$ \\
\hline${ }^{70} \mathrm{Ge}$ & $\leftrightarrow$ & ${ }^{88} \mathrm{Zn}$ & $93.5 \pm 0.3 \pm 0.8$ \\
\hline${ }^{75} \mathrm{As}$ & $\leftrightarrow$ & ${ }^{74} \mathrm{Ge}$ & $29.2 \pm 0.2 \pm 0.8$ \\
\hline${ }^{78} \mathrm{Se}$ & $\leftrightarrow$ & ${ }^{74} \mathrm{Ge}$ & $84.4 \pm 0.2 \pm 1.0$ \\
\hline${ }^{70} \mathrm{Br}$ & $\leftrightarrow$ & ${ }^{78} \mathrm{Se}$ & $28.7 \pm 0.2 \pm 0.8$ \\
\hline${ }^{81} \mathrm{Br}$ & $\leftrightarrow$ & ${ }^{80} \mathrm{Se}$ & $25.3 \pm 0.2 \pm 0.7$ \\
\hline${ }^{82} \mathrm{Kr}$ & $\leftrightarrow$ & ${ }^{80} \mathrm{Se}$ & $65.7 \pm 0.3 \pm 0.8$ \\
\hline${ }^{87} R b$ & $\leftrightarrow$ & ${ }^{86} \mathrm{Kr}$ & $18.2 \pm 0.3 \pm 0.6$ \\
\hline${ }^{88} S r$ & $\leftrightarrow$ & ${ }^{86} \mathrm{Kr}$ & $49.7 \pm 0.2 \pm 0.7$ \\
\hline${ }^{89} Y$ & $\leftrightarrow$ & ${ }^{88} \mathrm{Sr}$ & $23.4 \pm 0.1 \pm 0.6$ \\
\hline${ }^{80} \mathrm{Zr}$ & $\leftrightarrow$ & ${ }^{88} \mathrm{Sr}$ & $58.4 \pm 0.1 \pm 0.6$ \\
\hline${ }^{93} \mathrm{Nb}$ & $\leftrightarrow$ & ${ }^{92} \mathrm{Zr}$ & $23.2 \pm 0.1 \pm 0.6$ \\
\hline${ }^{94} \mathrm{Mo}$ & $\leftrightarrow$ & ${ }^{92} \mathrm{Zr}$ & $59.2 \pm 0.1 \pm 0.7$ \\
\hline${ }^{102} R u$ & $\leftrightarrow$ & ${ }^{100} M o$ & $46.4 \pm 0.2 \pm 0.8$ \\
\hline${ }^{103} R h$ & $\leftrightarrow$ & ${ }^{102} R u$ & $15.7 \pm 0.2 \pm 0.7$ \\
\hline${ }^{104} \mathrm{Pd}$ & $\leftrightarrow$ & ${ }^{102} R u$ & $34.4 \pm 0.5 \pm 0.7$ \\
\hline${ }^{109} \mathrm{Ag}$ & $\leftrightarrow$ & ${ }^{108} P d$ & $10.1 \pm 0.2 \pm 0.7$ \\
\hline${ }^{110} \mathrm{Cd}$ & $\leftrightarrow$ & ${ }^{108} P d$ & $21.0 \pm 0.2 \pm 0.7$ \\
\hline${ }^{118} \mathrm{In}$ & $\leftrightarrow$ & ${ }^{114} C d^{\star}$ & $4.9 \pm 0.2 \pm 0.7$ \\
\hline
\end{tabular}


TABLE VII. Differences of Barrett Radii for Isotones, $8 \leqslant Z \leqslant 126$ See page 194 for Explanation of Tables

\begin{tabular}{|c|c|c|c|}
\hline \multicolumn{3}{|c|}{ Isotone Pair } & $\Delta F_{h a}^{\mu}[a m]$ \\
\hline${ }^{116} S n$ & $\leftrightarrow$ & ${ }^{114} C d^{\star}$ & $18.2 \pm 0.2 \pm 0.6$ \\
\hline${ }^{124} \mathrm{Te}$ & $\leftrightarrow$ & ${ }^{123} \mathrm{Sb}$ & $38.0 \pm 0.2 \pm 0.6$ \\
\hline${ }^{124} \mathrm{Te}$ & $\leftrightarrow$ & ${ }^{122} S n^{\star}$ & $66.1 \pm 0.4 \pm 0.6$ \\
\hline${ }^{127} I$ & $\leftrightarrow$ & ${ }^{120} \mathrm{Te}$ & $27.9 \pm 0.1 \pm 0.5$ \\
\hline${ }^{128} X e$ & $\leftrightarrow$ & ${ }^{136} \mathrm{Te}$ & $61.1 \pm 0.3 \pm 0.5$ \\
\hline${ }^{133} \mathrm{Cs}$ & $\leftrightarrow$ & ${ }^{132} X e$ & $22.0 \pm 0.1 \pm 0.5$ \\
\hline${ }^{134} \mathrm{Ba}$ & $\leftrightarrow$ & ${ }^{132} \mathrm{Xe}$ & $53.8 \pm 0.1 \pm 0.5$ \\
\hline${ }^{139} \mathrm{La}$ & $\leftrightarrow$ & ${ }^{138} \mathrm{Ba}$ & $19.7 \pm 0.1 \pm 0.5$ \\
\hline${ }^{140} \mathrm{Ce}$ & $\leftrightarrow$ & ${ }^{138} B a$ & $47.9 \pm 0.1 \pm 0.5$ \\
\hline${ }^{141} \mathrm{Pr}$ & $\leftrightarrow$ & ${ }^{140} \mathrm{Ce}$ & $18.7 \pm 0.1 \pm 0.5$ \\
\hline${ }^{142} N d$ & $\leftrightarrow$ & ${ }^{140} \mathrm{Ce}$ & $45.7 \pm 0.1 \pm 0.5$ \\
\hline${ }^{144} \mathrm{Sm}$ & $\leftrightarrow$ & ${ }^{142} N d$ & $42.9 \pm 0.2 \pm 0.5$ \\
\hline${ }^{183} \mathrm{Eu}$ & $\leftrightarrow$ & ${ }^{152} \mathrm{Sm}$ & $36.6 \pm 0.1 \pm 0.8$ \\
\hline${ }^{164} \mathrm{Gd}$ & $\leftrightarrow$ & ${ }^{162} \mathrm{Sm}$ & $43.0 \pm 0.7 \pm 0.8$ \\
\hline${ }^{162} D y$ & $\leftrightarrow$ & ${ }^{100} G d$ & $36.0 \pm 0.4 \pm 0.8$ \\
\hline${ }^{160} \mathrm{Er}$ & $\leftrightarrow$ & ${ }^{164} D y$ & $35.7 \pm 0.4 \pm 0.7$ \\
\hline${ }^{170} \mathrm{Hf}$ & $\leftrightarrow$ & ${ }^{174} Y b$ & $25.5 \pm 0.9 \pm 0.7$ \\
\hline${ }^{181} \mathrm{Ta}$ & $\leftrightarrow$ & ${ }^{180} H f$ & $12.4 \pm 0.3 \pm 0.7$ \\
\hline${ }^{182} W$ & $\leftrightarrow$ & ${ }^{180} H f$ & $22.5 \pm 0.3 \pm 0.7$ \\
\hline${ }^{188} \mathrm{Os}$ & $\leftrightarrow$ & ${ }^{180} W^{\star}$ & $20.6 \pm 1.0 \pm 0.8$ \\
\hline${ }^{184} P t$ & $\leftrightarrow$ & ${ }^{182} \mathrm{Os}^{*}$ & $20.9 \pm 0.8 \pm 0.8$ \\
\hline
\end{tabular}

\begin{tabular}{cccc|}
\hline Isotone Pair & $\Delta R_{\mathrm{ka}}^{\mathrm{s}}[\mathrm{am}]$ \\
\hline${ }^{19 \gamma} \mathrm{Au}$ & $\leftrightarrow$ & ${ }^{190} \mathrm{Pt}$ & $5.7 \pm 0.2 \pm 0.8$ \\
${ }^{198} \mathrm{Hg}$ & $\leftrightarrow$ & ${ }^{196} \mathrm{Pt}^{\star}$ & $19.1 \pm 0.5 \pm 0.5$ \\
${ }^{203} \mathrm{Pl}$ & $\leftrightarrow$ & ${ }^{202} \mathrm{Hg}^{\star}$ & $6.1 \pm 1.1 \pm 0.5$ \\
${ }^{206} \mathrm{~Pb}$ & $\leftrightarrow$ & ${ }^{204} \mathrm{Hg}^{\star}$ & $17.6 \pm 0.6 \pm 0.3$ \\
${ }^{200} \mathrm{Bi}$ & $\leftrightarrow$ & ${ }^{208} \mathrm{~Pb}^{\star}$ & $18.8 \pm 3.0 \pm 0.5$ \\
\hline
\end{tabular}


TABLE VIII. Charge Density Distribution Parameters from Elastic Electron Scattering See page 194 for Explanation of Tables

\begin{tabular}{|c|c|c|c|c|c|c|c|}
\hline Nucleus & model & $\begin{array}{c}\left\langle r^{2}\right\rangle^{1 / 2} \\
{[\mathrm{fm}]}\end{array}$ & $\begin{array}{c}c \\
{[\mathrm{fm}]}\end{array}$ & $\stackrel{a}{a}$ & $\mathbf{w}$ & $\begin{array}{l}\text { q-range } \\
{\left[\mathrm{fm}^{-1}\right]}\end{array}$ & Ref. \\
\hline${ }^{12} \mathrm{C}$ & $\mathrm{FB}$ & $2.4776(55)$ & & & & $1.0-2.3$ & [Of91] \\
\hline${ }^{15} N$ & FB & $2.612(9)$ & & & & $0.4-3.2$ & [Vr88] \\
\hline${ }^{20} \mathrm{Mg}$ & $\mathrm{FB}$ & $2.960(4)$ & & & & $0.6-3.0$ & [So88] \\
\hline${ }^{30} S_{i}$ & FB & $3.193(13)$ & & & & $0.27-2.64$ & [We93] \\
\hline${ }^{31} P$ & FB & $3.191(5)$ & & & & $0.27-2.83$ & [We93] \\
\hline${ }^{32} S$ & FB & $3.230(5)$ & & & & $0.32-3.67$ & [We93] \\
\hline${ }^{70} G e^{\star}$ & $2 \mathrm{pF}$ & $4.055(8)$ & $4.430(8)$ & $0.5807(30)$ & & $0.59-1.50$ & [Kh90] \\
\hline${ }^{72} G e^{\star}$ & $2 \mathrm{pF}$ & $4.088(8)$ & $4.446(8)$ & $0.5921(30)$ & & $0.59-1.50$ & [Kh90] \\
\hline${ }^{74} G e^{\star}$ & $2 p F$ & $4.126(8)$ & $4.454(8)$ & $0.6085(30)$ & & $0.59-1.44$ & [Kh90] \\
\hline${ }^{70} G e^{\star}$ & $2 p F$ & $4.127(8)$ & $4.547(8)$ & $0.5780(30)$ & & $0.59-1.44$ & [Kh90] \\
\hline \multirow[t]{4}{*}{${ }^{74} \mathrm{Se}$} & $2 \mathrm{pF}$ & $4.07(2)$ & $4.387(22)$ & $0.6078(7)$ & & $0.42-1.47$ & [Kh88] \\
\hline & $3 p F$ & $4.05(2)$ & $4.488(67)$ & $0.5933(9)$ & $-0.09(6)$ & $0.42-1.47$ & [Kh88] \\
\hline & $2 \mathrm{pG}$ & $4.00(2)$ & $4.278(29)$ & $2.493(16)$ & & $0.42-1.47$ & [Kh88] \\
\hline & $3 \mathrm{pG}$ & $4.04(1)$ & $3.838(185)$ & $2.536(21)$ & $0.534(101)$ & $0.42-1.47$ & [Kh88] \\
\hline \multirow[t]{4}{*}{${ }^{79} \mathrm{Se}$} & $2 \mathrm{pF}$ & $4.162(10)$ & $4.471(11)$ & $0.6208(39)$ & & $0.42-1.6$ & [Kh88] \\
\hline & $3 \mathrm{pF}$ & $4.152(9)$ & $4.512(34)$ & $0.6189(43)$ & $-0.039(31)$ & $0.42-1.6$ & [Kh88 \\
\hline & $2 p G$ & $4.088(5)$ & $4.350(16)$ & $2.554(10)$ & & $0.42-1.6$ & [Kh88] \\
\hline & $3 p G$ & $4.133(7)$ & $3.682(93)$ & $2.608(10)$ & $0.645(38)$ & $0.42-1.6$ & [Kh88] \\
\hline \multirow[t]{4}{*}{${ }^{7 \mathrm{Se}}$} & $2 \mathrm{pF}$ & $4.138(14)$ & $4.581(18)$ & $0.5729(41)$ & & $0.42-1.68$ & [Kh8 \\
\hline & $3 p F$ & $4.123(9)$ & $4.634(34)$ & $0.5673(53)$ & $-0.058(33)$ & $0.42-1.68$ & [Kh8 \\
\hline & $2 \mathrm{pG}$ & $4.077(5)$ & $4.503(14)$ & $2.451(9)$ & & $0.42-1.68$ & [Kh8 \\
\hline & $3 p G$ & $4.111(7)$ & $4.099(73)$ & $2.512(13)$ & $0.559(48)$ & $0.42-1.68$ & [Kh88] \\
\hline \multirow[t]{4}{*}{${ }^{80} \mathrm{Se}$} & $2 \mathrm{pF}$ & $4.124(10)$ & $4.667(10)$ & $0.5339(42)$ & & $0.42-1.68$ & [Kh88] \\
\hline & $3 \mathrm{pF}$ & $4.137(9)$ & $4.614(33)$ & $0.5410(58)$ & $0.061(37)$ & $0.42-1.68$ & {$[\mathrm{Kh} 8$} \\
\hline & $2 \mathrm{pG}$ & $4.076(5)$ & $4.622(13)$ & $2.365(9)$ & & $0.42-1.68$ & [Kh88] \\
\hline & $3 p G$ & $4.122(7)$ & $4.139(68)$ & $2.405(14)$ & $0.657(44)$ & $0.42-1.68$ & [Kh88] \\
\hline
\end{tabular}

^ For the Ge isotopes measurements with an extended $q$ range are given by Mallot [Ma85] 
TABLE VIII. Charge Density Distribution Parameters from Elastic Electron Scattering See page 194 for Explanation of Tables

\begin{tabular}{|c|c|c|c|c|c|c|c|}
\hline Nucleus & model & $\begin{array}{c}\left\langle r^{2}\right\rangle^{1 / 2} \\
{[\mathrm{fm}]}\end{array}$ & $\underset{[\mathrm{fm}}{\mathrm{c}}]$ & $\stackrel{a}{[\mathrm{fm}]}$ & w & $\begin{array}{l}\text { q-range } \\
{\left[\mathrm{fm}^{-1}\right]}\end{array}$ & Ref. \\
\hline \multirow[t]{4}{*}{${ }^{12} \mathrm{Se}$} & $2 \mathrm{pF}$ & $4.118(11)$ & $4.718(11)$ & $0.5102(49)$ & & $0.42-1.6$ & [Kh88] \\
\hline & $3 p F$ & $4.134(9)$ & $4.642(37)$ & $0.5225(72)$ & $0.089(43)$ & $0.42-1.6$ & [Kh88] \\
\hline & $2 \mathrm{pG}$ & $4.076(10)$ & $4.694(13)$ & 2.307 (11) & & $0.42-1.6$ & [Kh88] \\
\hline & $3 p G$ & $4.122(8)$ & $4.212(73)$ & $2.429(17)$ & $0.675(49)$ & $0.42-1.6$ & [Kh88] \\
\hline${ }^{80} \mathrm{Zr}$ & FB & $4.280(10)$ & & & & $0.5-2.5$ & [Ma89] \\
\hline${ }^{22} \mathrm{Zr}$ & FB & $4.312(11)$ & & & & $0.5-2.5$ & [Ma89] \\
\hline${ }^{94} \mathrm{Zr}$ & FB & $4.332(11)$ & & & & $0.5-2.5$ & [Ma89] \\
\hline${ }^{22} \mathrm{Mo}$ & FB & $4.306(10)$ & & & & $0.56-1.96$ & [Ma89] \\
\hline${ }^{84} \mathrm{Mo}$ & FB & $4.346(10)$ & & & & $0.56-1.96$ & [Ma89] \\
\hline${ }^{90} \mathrm{Mo}$ & FB & $4.377(10)$ & & & & $0.56-1.96$ & [Ma89] \\
\hline${ }^{98} \mathrm{Mo}$ & FB & $4.408(10)$ & & & & $0.56-1.96$ & [Ma89] \\
\hline${ }^{100} \mathrm{Mo}$ & FB & $4.447(10)$ & & & & $0.56-1.96$ & [Ma89] \\
\hline${ }^{188} \mathrm{Os}$ & SOG & $5.4001(13)$ & & & & $0.6-3.2$ & [Bo88] \\
\hline${ }^{100} \mathrm{Os}$ & SOG & $5.4062(14)$ & & & & $0.6-3.2$ & [Bo88] \\
\hline${ }^{192} \mathrm{Os}$ & SOG & $5.410(1)$ & & & & $0.6-3.2$ & [Bo88] \\
\hline${ }^{10.9} \mathrm{Pt}$ & SOG & $5.369(9)$ & & & & $0.6-3.2$ & [Bo88] \\
\hline${ }^{186} \mathrm{Pt}$ & SOG & $5.370(3)$ & & & & $0.6-3.2$ & [Bo88] \\
\hline${ }^{108} \mathrm{Hg}$ & FB & $5.439(4)$ & & & & $0.43-2.93$ & [La86] \\
\hline${ }^{204} \mathrm{Hg}$ & FB & $5.4717(16)$ & & & & $0.42-2.88$ & [Bu89] \\
\hline${ }^{204} \mathrm{~Pb}$ & FB & $5.4603(40)$ & & & & $0.51-2.24$ & [Ma92a] \\
\hline${ }^{200} \mathrm{~Pb}$ & FB & $5.4742(41)$ & & & & $0.51-2.24$ & [Ma92a] \\
\hline${ }^{207} \mathrm{~Pb}$ & FB & $5.4838(41)$ & & & & $0.51-2.24$ & [Ma92a] \\
\hline${ }^{208} \mathrm{~Pb}$ & FB & $5.4785(41)$ & & & & $0.51-2.24$ & [Ma92a] \\
\hline
\end{tabular}


TABLE IX. Fourier-Bessel Coefficients from Elastic Electron Scattering See page 194 for Explanation of Tables

\begin{tabular}{|c|c|c|c|c|c|}
\hline Nucleus & ${ }^{12} \mathrm{C}$ & ${ }^{16} N$ & ${ }^{26} \mathrm{Mg}$ & ${ }^{30} \mathrm{Si}$ & ${ }^{31} P$ \\
\hline$\left(r^{2}\right)^{1 / 2}(\mathrm{fm})$ & 2.4777 & 2.6130 & 2.9610 & 3.1932 & 3.1913 \\
\hline$\left(r^{4}\right)^{1 / 4}(\mathrm{fm})$ & 2.7722 & 2.9689 & 3.2339 & 3.5507 & 3.5241 \\
\hline$\left\langle r^{0}\right\rangle^{1 / 0}(\mathrm{fm})$ & 3.0397 & 3.3164 & 3.4594 & 3.8977 & 3.8251 \\
\hline$R_{k \alpha}^{e}(\mathrm{fm})$ & 3.1915 & & 3.8103 & 4.0994 & 4.0983 \\
\hline a1 & $1.5709 \mathrm{e}-2$ & $2.5490 \mathrm{e}-2$ & $2.9280 \mathrm{e}-2$ & $2.8322 \mathrm{e}-2$ & $3.5280 \mathrm{e}-2$ \\
\hline a2 & $3.8610 \mathrm{e}-2$ & $5.0630 \mathrm{e}-2$ & $5.5520 \mathrm{e}-2$ & $5.3722 \mathrm{e}-2$ & $5.9545 \mathrm{e}-2$ \\
\hline $\mathbf{a 3}$ & $3.6418 \mathrm{e}-2$ & $2.9840 \mathrm{e}-2$ & $2.3910 \mathrm{e}-2$ & $2.4661 \mathrm{e}-2$ & $1.7241 \mathrm{e}-2$ \\
\hline a4 & $1.4293 \mathrm{e}-2$ & $-5.5300 \mathrm{e}-3$ & $-1.3940 \mathrm{e}-2$ & $-1.2888 \mathrm{e}-2$ & $-1.9338 \mathrm{e}-2$ \\
\hline a5 & $-4.4628 \mathrm{e}-3$ & $-1.5900 \mathrm{e}-2$ & $-1.5840 \mathrm{e}-2$ & $-1.7112 \mathrm{e}-2$ & $-1.3171 \mathrm{e}-2$ \\
\hline a6 & $-9.8420 \mathrm{e}-3$ & $-7.7000 \mathrm{e}-3$ & $-3.5680 \mathrm{e}-3$ & $-4.4233 \mathrm{e}-3$ & $1.4065 \mathrm{e}-3$ \\
\hline a7 & $-6.6518 \mathrm{e}-3$ & $-2.3000 \mathrm{e}-3$ & $1.9670 \mathrm{e}-3$ & $3.2357 \mathrm{e}-3$ & $3.6747 \mathrm{e}-3$ \\
\hline a8 & $-2.7066 \mathrm{e}-3$ & $-4.0000 \mathrm{e}-4$ & $1.4470 \mathrm{e}-3$ & $2.2195 \mathrm{e}-3$ & $6.3926 \mathrm{e}-4$ \\
\hline a9 & $-5.6697 \mathrm{e}-4$ & & $-8.6820 \mathrm{e}-5$ & $4.0898 \mathrm{e}-4$ & $-3.2297 e-4$ \\
\hline a10 & $-2.7453 e-4$ & & $-1.4000 \mathrm{e}-4$ & $2.0363 \mathrm{e}-4$ & $1.8286 \mathrm{e}-4$ \\
\hline a11 & $-1.7093 \mathrm{e}-4$ & & $7.0000 \mathrm{e}-5$ & $-2.6112 \mathrm{e}-4$ & $-1.0781 e-4$ \\
\hline a12 & $1.2433 \mathrm{e}-4$ & & $-2.0000 \mathrm{e}-5$ & $2.3104 \mathrm{e}-4$ & $6.6628 \mathrm{e}-5$ \\
\hline a13 & $-4.8496 e-5$ & & $3.0000 \mathrm{e}-6$ & $-1.8855 \mathrm{e}-4$ & $-4.2991 \mathrm{e}-5$ \\
\hline a14 & $1.5675 \mathrm{e}-5$ & & $1.0000 \mathrm{e}-7$ & & $2.8800 e-5$ \\
\hline a15 & $-4.5194 \mathrm{e}-6$ & & $-2.0000 \mathrm{e}-7$ & & $-1.9932 e-5$ \\
\hline a16 & $1.1920 \mathrm{e}-6$ & & & & \\
\hline a17 & $-2.9065 e-7$ & & & & \\
\hline a18 & $6.5845 \mathrm{e}-8$ & & & & \\
\hline$R_{\text {cut }}(\mathrm{fm})$ & 8.0 & 7.0 & 8.0 & 8.5 & 8.0 \\
\hline Ref. & [Of91] & [Vr88] & [So88] & [We9:3] & [We9:3] \\
\hline
\end{tabular}


G. FRICKE et al. Nuclear Charge Radii

TABLE IX. Fourier-Bessel Coefficients from Elastic Electron Scattering See page 194 for Explanation of Tables

\begin{tabular}{|c|c|c|c|c|c|}
\hline Nucleus & ${ }^{32} S$ & ${ }^{\circ 0} \mathrm{Zr}$ & ${ }^{92} Z r$ & ${ }^{94} Z r$ & ${ }^{92} \mathrm{Mo}$ \\
\hline$\left(r^{2}\right)^{1 / 2}(\mathrm{fm})$ & 3.2295 & 4.2796 & 4.3118 & 4.3319 & 4.3058 \\
\hline$\left(r^{4}\right)^{1 / 4}(\mathrm{fm})$ & 3.5439 & 4.6035 & 4.6399 & 4.6619 & 4.6253 \\
\hline$\left\langle r^{6}\right\rangle^{3 / 6}(\mathrm{fm})$ & 3.8086 & 4.8695 & 4.9091 & 4.9313 & 4.8884 \\
\hline$R_{\text {ka }}^{e}(\mathrm{fm})$ & 4.1483 & 5.4808 & 5.5212 & 5.5465 & 5.5149 \\
\hline a1 & $3.7359 \mathrm{e}-2$ & $1.4655 \mathrm{e}-0$ & $1.4585 \mathrm{e}-0$ & $1.4541 \mathrm{e}-0$ & $1.4087 \mathrm{e}-0$ \\
\hline a2 & $6.0918 \mathrm{e}-2$ & $-4.8451 \mathrm{e}-1$ & $-4.7274 \mathrm{e}-1$ & $-4.6543 \mathrm{e}-1$ & $-3.8490 \mathrm{e}-1$ \\
\hline a3 & $1.5029 \mathrm{e}-2$ & $-4.5590 \mathrm{e}-2$ & $-4.8662 \mathrm{e}-2$ & $-4.9800 \mathrm{e}-2$ & $-8.1355 e-2$ \\
\hline a4 & $-1.8758 \mathrm{e}-2$ & $7.3069 \mathrm{e}-2$ & $6.9795 \mathrm{e}-2$ & $6.6551 \mathrm{e}-2$ & $5.3179 \mathrm{e}-2$ \\
\hline $\mathbf{a 5}$ & $-1.0174 \mathrm{e}-2$ & $2.7921 \mathrm{e}-3$ & $3.6133 \mathrm{e}-3$ & $4.6089 \mathrm{e}-3$ & $1.3497 \mathrm{e}-2$ \\
\hline $\mathbf{a 6}$ & $3.1107 \mathrm{e}-3$ & $-1.3480 e-2$ & $-1.2283 \mathrm{e}-2$ & $-1.1399 \mathrm{e}-2$ & $-7.3112 \mathrm{e}-3$ \\
\hline a7 & $3.9209 \mathrm{e}-3$ & $-3.6792 \mathrm{e}-4$ & $-4.4525 e-4$ & $-9.2051 e-4$ & $-2.7433 \mathrm{e}-3$ \\
\hline $\mathbf{a} 8$ & $1.2556 \mathrm{e}-3$ & $3.0278 \mathrm{e}-3$ & $2.6520 \mathrm{e}-3$ & $2.8621 \mathrm{e}-3$ & $7.0109 \mathrm{e}-4$ \\
\hline $\mathbf{a} 9$ & $-1.1683 \mathrm{e}-4$ & $-1.4165 e-5$ & $-2.3637 \mathrm{e}-4$ & $-3.5979 \mathrm{e}-4$ & $1.0278 \mathrm{e}-4$ \\
\hline a10 & $-6.6587 e-4$ & $-3.1561 \mathrm{e}-4$ & $-1.2070 \mathrm{e}-4$ & $-1.3554 \mathrm{e}-4$ & $9.3746 \mathrm{e}-5$ \\
\hline a11 & $-1.7395 e-4$ & $-1.3736 e-4$ & $-7.1995 e-5$ & $-7.2113 \mathrm{e}-5$ & $4.5421 \mathrm{e}-5$ \\
\hline a12 & $5.6504 \mathrm{e}-5$ & $-2.2203 \mathbf{e}-5$ & $-2.1952 \mathrm{e}-5$ & $-1.8146 e-5$ & $1.8367 e-5$ \\
\hline a13 & $-1.1374 \mathrm{e}-4$ & $1.1254 \mathrm{e}-5$ & $-1.8051 \mathrm{e}-6$ & $1.9590 \mathrm{e}-6$ & $6.7518 \mathrm{e}-6$ \\
\hline a14 & $9.9554 \mathrm{e}-5$ & $1.2484 \mathrm{e}-5$ & $2.6161 \mathrm{e}-6$ & $5.0940 \mathrm{e}-6$ & $2.3267 \mathrm{e}-6$ \\
\hline a15 & $-6.2021 e-5$ & $7.3383 \mathrm{e}-5$ & $2.2102 \mathrm{e}-6$ & $3.5240 \mathrm{e}-6$ & $7.6142 \mathrm{e}-6$ \\
\hline$R_{\text {cut }}(\mathbf{f m})$ & 8.0 & 10.0 & 10.0 & 10.0 & 9.5 \\
\hline Ref. & [We9:3] & [Mas9] & [Ma89] & [Ma89] & [Ma89] \\
\hline
\end{tabular}


TABLE IX. Fourier-Bessel Coefficients from Elastic Electron Scattering See page 194 for Explanation of Tables

\begin{tabular}{|c|c|c|c|c|c|}
\hline Nucleus & ${ }^{94} M o$ & ${ }^{\circ 6} \mathrm{Mo}$ & ${ }^{80} \mathrm{Mo}$ & ${ }^{100} \mathrm{Mo}$ & ${ }^{190} \mathrm{Hg}$ \\
\hline$\left\langle r^{2}\right\rangle^{1 / 2}(\mathrm{fm})$ & 4.3462 & 4.3766 & 4.4077 & 4.4466 & 5.4369 \\
\hline$\left\langle r^{4}\right)^{1 / 4}(\mathrm{fm})$ & 4.6719 & 4.7061 & 4.7417 & 4.7852 & 5.7615 \\
\hline$\left\langle r^{6}\right\rangle^{1 / 6}(\mathrm{fm})$ & 4.9407 & 4.9765 & 5.0167 & 5.0657 & 6.0079 \\
\hline$R_{h \alpha}^{e}(\mathrm{fm})$ & 5.5651 & 5.6032 & 5.6420 & 5.6911 & 6.9616 \\
\hline a1 & $1.3993 \mathrm{e}-0$ & $1.3921 \mathrm{e}-0$ & $1.3847 \mathrm{e}-0$ & $1.3755 \mathrm{e}-0$ & $0.7763 \mathrm{e}-0$ \\
\hline a2 & $-3.7117 e-1$ & $-3.6096 \mathrm{e}-1$ & $-3.5036 e-1$ & $-3.3662 e-1$ & $5.0020 \mathrm{e}-1$ \\
\hline a3 & $-8.2942 \mathrm{e}-2$ & $-8.2924 \mathrm{e}-2$ & $-8.4221 \mathrm{e}-2$ & $-8.7092 \mathrm{e}-2$ & $-7.0634 \mathrm{e}-1$ \\
\hline a4 & $4.9823 \mathrm{e}-2$ & $4.6040 \mathrm{e}-2$ & $4.3079 \mathrm{e}-2$ & $4.0659 \mathrm{e}-2$ & $-0.7791 e-1$ \\
\hline a5 & $1.3324 \mathrm{e}-2$ & $1.3252 \mathrm{e}-2$ & $1.3505 \mathrm{e}-2$ & $1.3770 \mathrm{e}-2$ & $4.0512 \mathrm{e}-1$ \\
\hline a6 & $-6.7303 e-3$ & $-5.9623 \mathrm{e}-3$ & $-4.7350 \mathrm{e}-3$ & $-4.3166 e-3$ & $-1.0145 \mathrm{e}-1$ \\
\hline a7 & $-2.5153 \mathrm{e}-3$ & $-2.4484 e-3$ & $-2.5760 \mathrm{e}-3$ & $-2.1707 e-3$ & $-1.3954 \mathrm{e}-1$ \\
\hline a8 & $7.6567 \mathrm{e}-4$ & $7.9107 \mathrm{e}-4$ & $3.7381 \mathrm{e}-4$ & $2.9343 \mathrm{e}-4$ & $0.7129 \mathrm{e}-1$ \\
\hline a9 & $1.0129 \mathrm{e}-4$ & $6.7532 \mathrm{e}-5$ & $9.9915 \mathrm{e}-5$ & $3.0507 \mathrm{e}-6$ & $0.1619 \mathrm{e}-1$ \\
\hline a10 & $5.1645 \mathrm{e}-5$ & $4.2222 \mathrm{e}-5$ & $6.4032 \mathrm{e}-5$ & $1.0859 \mathrm{e}-5$ & $-0.2056 \mathrm{e}-1$ \\
\hline a11 & $2.2401 \mathrm{e}-5$ & $2.0117 \mathrm{e}-5$ & $2.8547 e-5$ & $7.1838 \mathrm{e}-6$ & \\
\hline a12 & $8.7445 e-6$ & $8.2364 \mathrm{e}-6$ & $1.1104 \mathrm{e}-5$ & $3.3711 \mathrm{e}-6$ & \\
\hline a13 & $3.1709 \mathrm{e}-6$ & $3.0688 \mathrm{e}-6$ & $3.9917 \mathrm{e}-6$ & $1.3520 \mathrm{e}-6$ & \\
\hline a14 & $1.0863 \mathrm{e}-6$ & $1.0694 \mathrm{e}-6$ & $1.3559 \mathrm{e}-6$ & $4.9322 \mathrm{e}-7$ & \\
\hline a15 & $3.5460 \mathrm{e}-6$ & $3.5305 \mathrm{e}-6$ & $4.3927 \mathrm{e}-6$ & $1.6799 \mathrm{e}-7$ & \\
\hline$R_{\text {cue }}(\mathrm{fm})$ & 9.5 & 9.5 & 9.5 & 9.5 & 11.0 \\
\hline Ref. & [Ma89] & [Ma89] & [Ma89] & [Ma89] & [La86] \\
\hline
\end{tabular}


TABLE IX. Fourier-Bessel Coefficients from Elastic Electron Scattering See page 194 for Explanation of Tables

\begin{tabular}{|c|c|c|c|c|c|}
\hline Nucleus & ${ }^{204} \mathrm{Hg}$ & ${ }^{204} \mathrm{~Pb}$ & ${ }^{200} \mathrm{~Pb}$ & ${ }^{207} \mathrm{~Pb}$ & ${ }^{208} \mathrm{~Pb}$ \\
\hline$\left\langle r^{2}\right\rangle^{1 / 2}(\mathrm{fm})$ & 5.4717 & 5.4603 & 5.4742 & 5.4838 & 5.4785 \\
\hline$\left\langle r^{4}\right\rangle^{1 / 4}(\mathrm{fm})$ & 5.8157 & 5.8034 & 5.8173 & 5.8280 & 5.8224 \\
\hline$\left\langle r^{\circ}\right\rangle^{1 / 6}(\mathrm{fm})$ & 6.0975 & 6.0721 & 6.0852 & 6.0978 & 6.0909 \\
\hline $\boldsymbol{R}_{k \alpha}(\mathrm{fm})$ & 6.9974 & 6.9787 & 6.9966 & 7.0088 & 7.0018 \\
\hline a1 & $5.0880 \mathrm{e}-2$ & $1.4429 \mathrm{e}-0$ & $1.4403 \mathrm{e}-0$ & $1.4386 \mathrm{e}-0$ & $1.4396 \mathrm{e}-0$ \\
\hline $\mathbf{a 2}$ & $5.0679 \mathrm{e}-2$ & $-4.2429 e-1$ & $-4.1971 e-1$ & $-4.1663 e-1$ & $-4.1850 \mathrm{e}-1$ \\
\hline a3 & $-3.9771 \mathrm{e}-2$ & $-9.5671 e-2$ & $-9.7085 e-2$ & $-9.8583 e-2$ & $-9.7163 e-2$ \\
\hline a4 & $-3.1403 e-2$ & $7.0018 \mathrm{e}-2$ & $6.8671 e-2$ & $6.8752 \mathrm{e}-2$ & $6.8006 e-2$ \\
\hline a5 & $2.8120 \mathrm{e}-2$ & $2.6037 \mathrm{e}-2$ & $2.6342 \mathrm{e}-2$ & $2.6388 e-2$ & $2.6476 \mathrm{e}-2$ \\
\hline $\mathbf{a 6}$ & $1.0580 \mathrm{e}-2$ & $-1.6179 e-2$ & $-1.5755 e-2$ & $-1.5632 \mathrm{e}-2$ & $-1.5307 e-2$ \\
\hline a7 & $-1.6402 \mathrm{e}-2$ & $-6.9630 \mathrm{e}-3$ & $-6.9078 e-3$ & $-6.8570 e-3$ & $-7.1246 \mathrm{e}-3$ \\
\hline $\mathbf{a 8}$ & $-3.1958 e-3$ & $2.8872 e-3$ & $2.9708 e-3$ & $2.5973 e-3$ & $2.7987 \mathrm{e}-3$ \\
\hline $\mathbf{a} \mathbf{9}$ & $6.9355 e-3$ & $2.4712 \mathrm{e}-3$ & $2.4830 \mathrm{e}-3$ & $2.4401 \mathrm{e}-3$ & $2.3767 e-3$ \\
\hline $\mathbf{a 1 0}$ & $7.0777 \mathrm{e}-4$ & $-1.0106 e-3$ & $-1.3911 \mathrm{e}-3$ & $-9.4205 e-4$ & $-1.0125 e-3$ \\
\hline a11 & $-1.4961 \mathrm{e}-4$ & $-2.5467 e-4$ & $1.5425 \mathrm{e}-4$ & $-2.2020 \mathrm{e}-4$ & $-2.5836 e-4$ \\
\hline a12 & $2.4032 e-4$ & $2.2412 \mathrm{e}-5$ & $1.3471 \mathrm{e}-4$ & $8.2610 \mathrm{e}-6$ & $6.4297 e-5$ \\
\hline a13 & $-2.9939 e-4$ & $4.8363 e-5$ & $7.5187 \mathrm{e}-5$ & $3.9685 \mathrm{e}-5$ & $6.5528 \mathrm{e}-5$ \\
\hline a14 & $2.0003 e-4$ & $1.9367 \mathrm{e}-5$ & $3.0809 e-6$ & $1.7911 \mathrm{e}-5$ & $1.4523 \mathrm{e}-5$ \\
\hline a15 & $-1.5870 \mathrm{e}-4$ & $-2.3405 e-6$ & $-2.4780 e-5$ & $-1.0828 e-6$ & $-1.4430 e-5$ \\
\hline$R_{\text {cut }}(\mathrm{fm})$ & 12.0 & 12.5 & 12.5 & 12.5 & 12.5 \\
\hline Ref. & [Bu89] & [Ma92a] & [Ma.92a] & [Ma92a] & [Ma92a] \\
\hline
\end{tabular}


TABLE X. King Plots: Optical versus Combined Muonic and Elastic Electron Scattering Data See page 194 for Explanation of Tables

20 CALCIUM
$\mathrm{R}_{\mathrm{k} \alpha}^{\mu}(40)=4.4628 \mathrm{fm}$
$\mathrm{C}_{2} / \mathrm{C}_{1}=-2.14 \cdot 10^{-4} \mathrm{fm}^{-2}$
$\mathrm{C}_{3} / \mathrm{C}_{1}=1.37 \cdot 10^{-6} \mathrm{fm}^{-4}$

$\begin{array}{rlrl}\lambda_{\text {proj }} & =2 \cdot 599 \mathrm{~nm} & F_{2 \times 599 \mathrm{~nm}}=-93.7(1.6) \frac{\mathrm{MHz}}{\mathrm{fm}^{2}} \\ \mathrm{FS}^{42-40}= & -18.8(.5) \mathrm{MHz} & \mathrm{NMS}^{42-40}=652.6 \mathrm{MHz} & \\ \mathrm{MS}^{42-40}= & 625.9(.1) \mathrm{MHz} & \mathrm{SMS}^{42-40} & =-26.7(.1) \mathrm{MHz} \\ \chi^{2} / \mathrm{D} . \mathrm{F} . & =0.84 & \mathrm{SMS} / \mathrm{NMS} & =-0.0409(.0002)\end{array}$

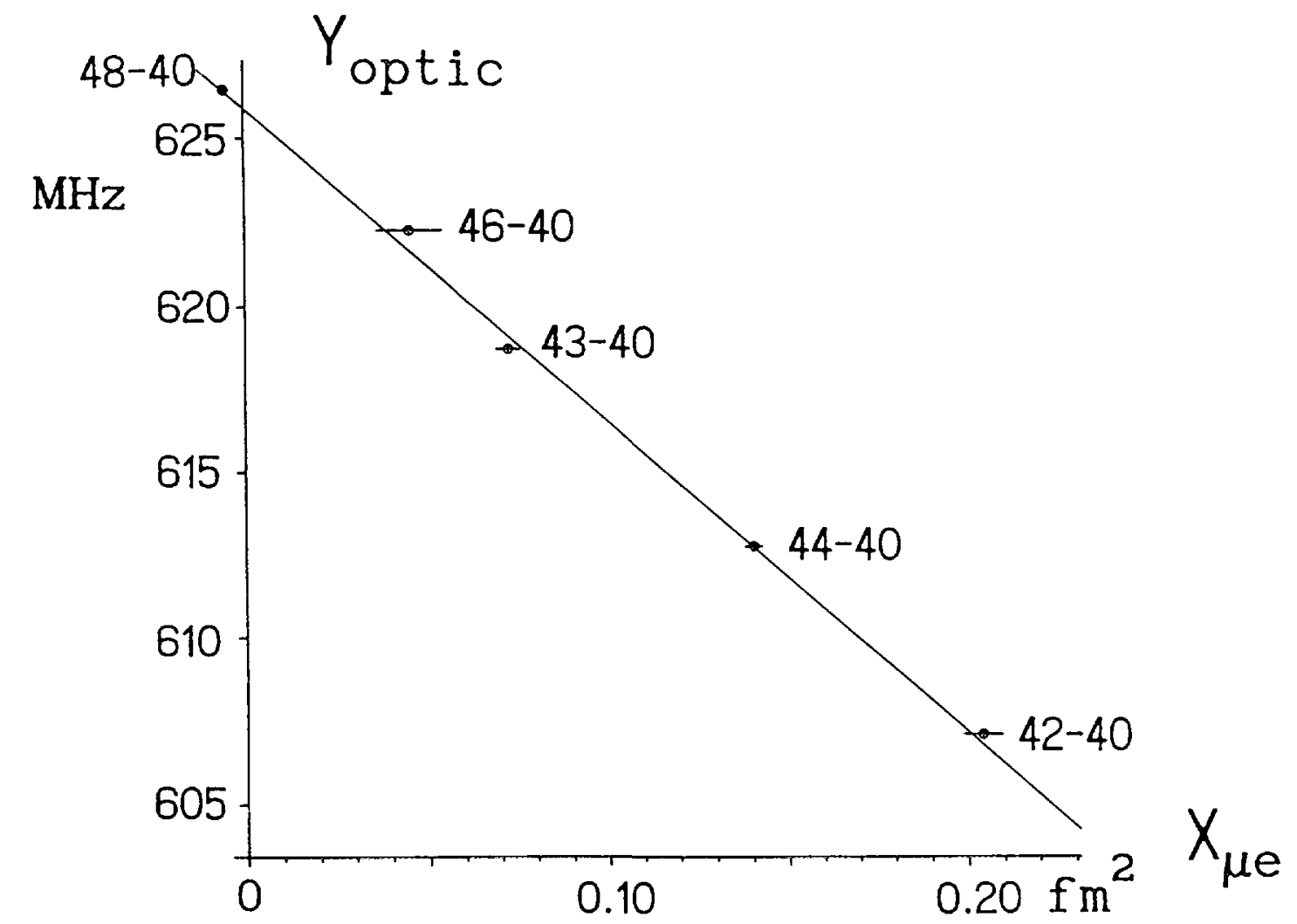

\begin{tabular}{|c|c|c|c|c|c|c|}
\hline Isotope & $V_{2}$ & $V_{4}$ & $v_{6}$ & $\begin{array}{c}\text { Isotope } \\
\text { pair }\end{array}$ & $\begin{array}{l}\delta<r^{2}>o \mu e \\
{\left[f m^{2}\right]}\end{array}$ & $\begin{array}{l}\mathrm{HM} \\
{[\%]}\end{array}$ \\
\hline $\begin{array}{l}40^{\circ} \\
42 \\
43 \\
44 \\
46 \\
48^{e}\end{array}$ & $\begin{array}{l}1.28364 \\
1.28400 \\
1.28410 \\
1.28430 \\
1.28460 \\
1.28493\end{array}$ & $\begin{array}{l}1.1776 \\
1.1799 \\
1.1811 \\
1.1823 \\
1.1846 \\
1.1870\end{array}$ & $\begin{array}{l}1.101 \\
1.105 \\
1.107 \\
1.109 \\
1.113 \\
1.117\end{array}$ & $\begin{array}{l}42-40 \\
43-40 \\
44-40 \\
46-40 \\
48-40\end{array}$ & $\begin{array}{r}0.202(4) \\
0.113(3) \\
0.269(5) \\
0.107(4) \\
-0.021(3)\end{array}$ & $\begin{array}{l}-0.50 \\
-0.32 \\
-0.45 \\
-0.14 \\
-4.47\end{array}$ \\
\hline
\end{tabular}


TABLE X. King Plots: Optical versus Combined Muonic and Elastic Electron Scattering Data See page 194 for Explanation of Tables

\begin{tabular}{|l|}
36 KRYPTON \\
$\mathrm{R}_{\mathrm{k} \alpha}^{\mu}(80)=5.3773 \mathrm{fm}$ \\
$\mathrm{C}_{2} / \mathrm{C}_{1}=-4.11 \cdot 10^{-4} \mathrm{fm}^{-2}$ \\
$\mathrm{C}_{3} / \mathrm{C}_{1}=1.47 \cdot 10^{-6} \mathrm{fm}^{-4}$
\end{tabular}

$\begin{array}{rlrl}\lambda_{\text {pros }} & =810.7 \mathrm{~nm} & F_{810.7 \mathrm{~nm}} & =-438(86) \frac{\mathrm{MHz}}{\mathrm{fm}^{2}} \\ \mathrm{FS}^{80-78} & =20.1(7.7) \mathrm{MHz} & \mathrm{NMS}^{80-78} & =65.2 \mathrm{MHz} \\ \mathrm{MS}^{80-78} & =59.9(3.9) \mathrm{MHz} & \mathrm{SMS}^{80-78} & =-5.2(3.9) \mathrm{MHz} \\ \chi^{2} / \mathrm{D} . \mathrm{F} . & =0.20 & \mathrm{SMS} / \mathrm{NMS} & =-0.08(.06)\end{array}$

\section{Yoptic}

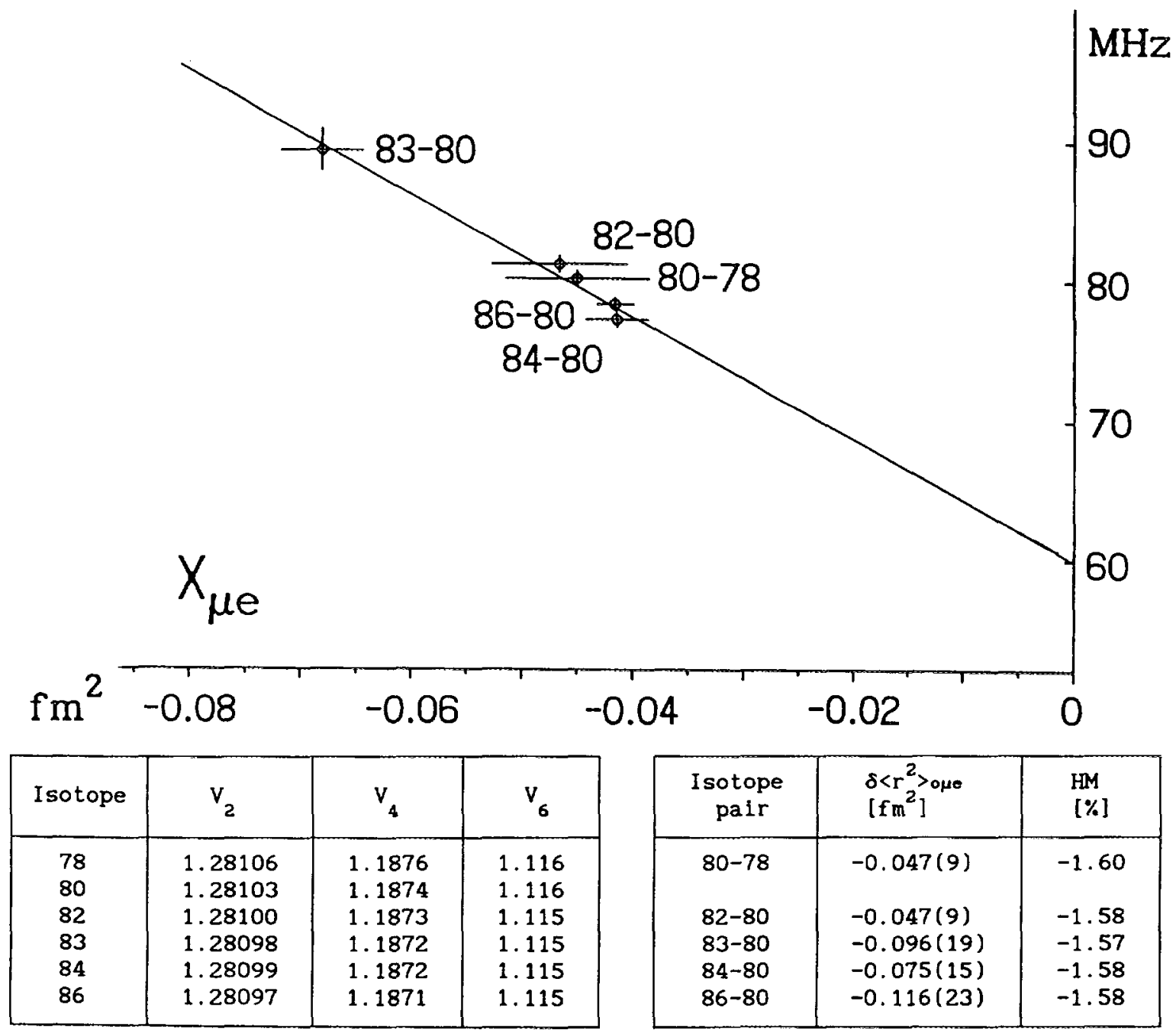

Note: The $V$ values are calculated with a two parameter Fermi distribution with the parameters taken from Table III-A. 
TABLE X. King Plots: Optical versus Combined Muonic and Elastic Electron Scattering Data See page 194 for Explanation of Tables

\begin{tabular}{|c|c|c|c|c|}
\hline 38 STRONTIUM & $\lambda_{\text {prof }}$ & $=293.2 \mathrm{~nm}$ & $\mathrm{~F}_{293.2 \mathrm{~nm}}$ & $=-611(81) \frac{\mathrm{MHz}}{\mathrm{fm}^{2}}$ \\
\hline $\begin{array}{l}\mathrm{R}_{\mathrm{k} \alpha}^{\mu}(88)=5.4092 \mathrm{fm} \\
\mathrm{C}_{2} / \mathrm{C}_{1}=-4.45 \cdot 10^{-4} \mathrm{fm}^{-2} \\
\mathrm{C}_{3} / \mathrm{C}_{1}=1.56 \cdot 10^{-6} \mathrm{fm}^{-4}\end{array}$ & $\begin{array}{l}\mathrm{FS}^{88-86} \\
\mathrm{MS}^{88-86} \\
\chi^{2} / \mathrm{D} \cdot \mathrm{F}\end{array}$ & $\begin{array}{l}=37.2(9.7) \mathrm{MHz} \\
=187.8(5.5) \mathrm{MHz} \\
=0.07\end{array}$ & $\begin{array}{l}\text { NMS }^{88-86} \\
\text { SMS }^{88-86} \\
\text { SMS/NMS }\end{array}$ & $\begin{array}{l}=148.4 \mathrm{MHz} \\
=39.5(5.5) \mathrm{MHz} \\
=0.27(0.04)\end{array}$ \\
\hline
\end{tabular}

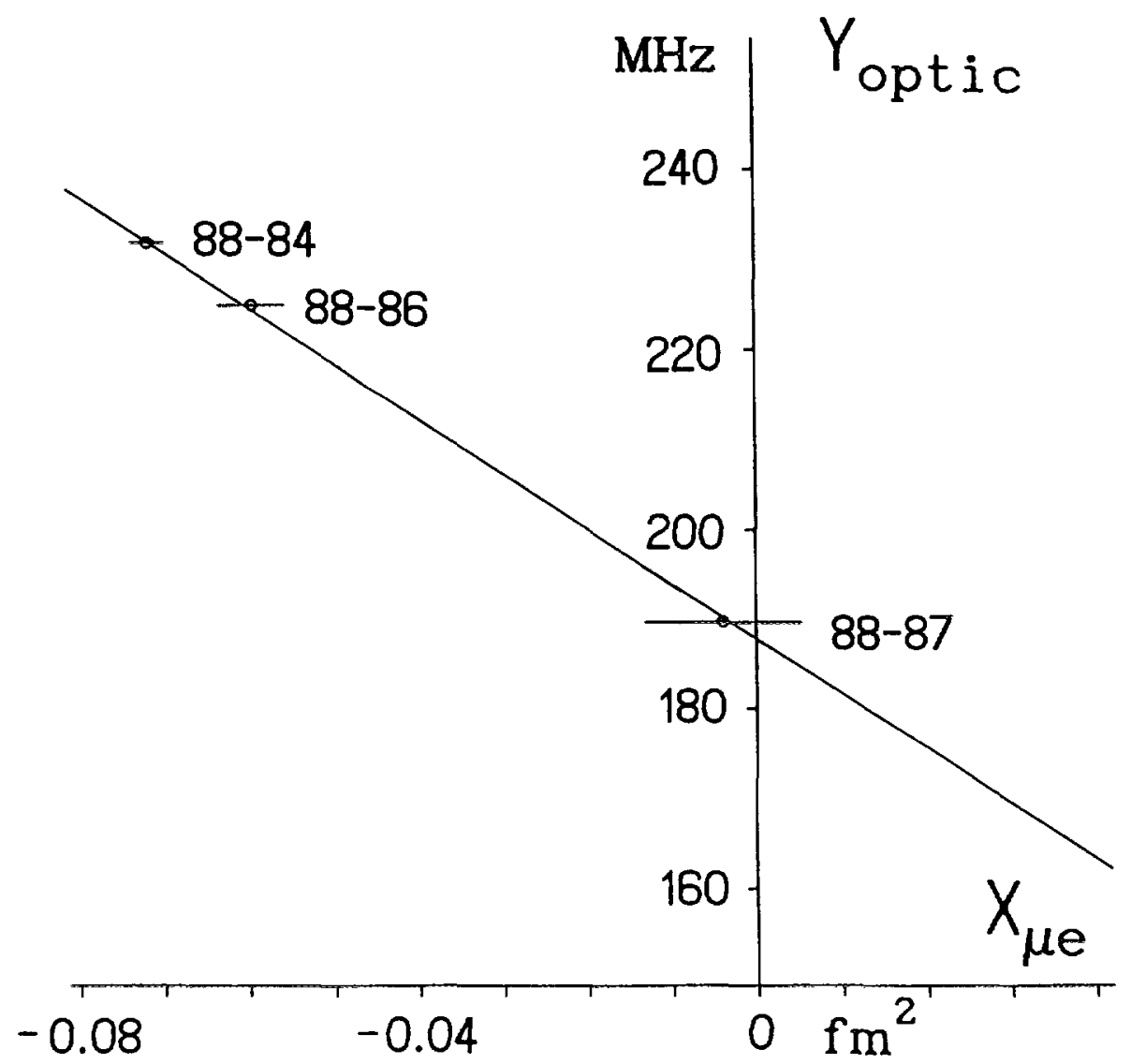

\begin{tabular}{|l|c|c|c|}
\hline Isotope & $V_{2}$ & $V_{4}$ & $V_{6}$ \\
\hline 84 & 1.28215 & 1.1952 & 1.134 \\
86 & 1.28215 & 1.1952 & 1.134 \\
87 & 1.28215 & 1.1952 & 1.134 \\
$88^{\circ}$ & 1.28215 & 1.1952 & 1.134 \\
\hline
\end{tabular}

\begin{tabular}{|c|l|c|}
\hline $\begin{array}{c}\text { Isotope } \\
\text { pair }\end{array}$ & $\begin{array}{l}\delta<\mathrm{r}^{2}>\text { ore } \\
{\left[\mathrm{fm}^{2}\right]}\end{array}$ & $\begin{array}{c}\mathrm{HM} \\
{[\%]}\end{array}$ \\
\hline $88-84$ & $-0.149(20)$ & -1.83 \\
$88-86$ & $-0.062(8)$ & -1.82 \\
$88-87$ & $-0.0016(2)$ & -1.87 \\
\hline
\end{tabular}

Note: The $V$ values for ${ }^{88} \mathrm{Sr}$ are taken from elastic electron scattering [Vr87]. The same $V$ values have been used for the other isotopes. 
TABLE X. King Plots: Optical versus Combined Muonic and Elastic Electron Scattering Data See page 194 for Explanation of Tables

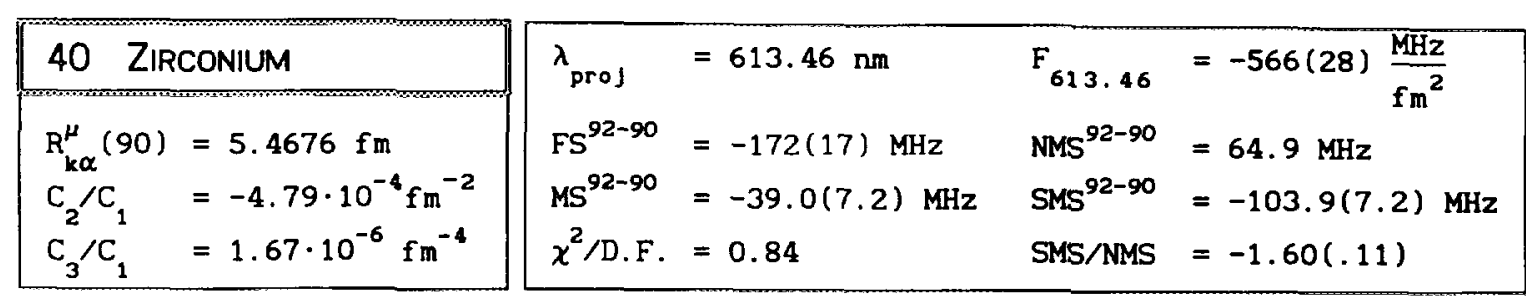

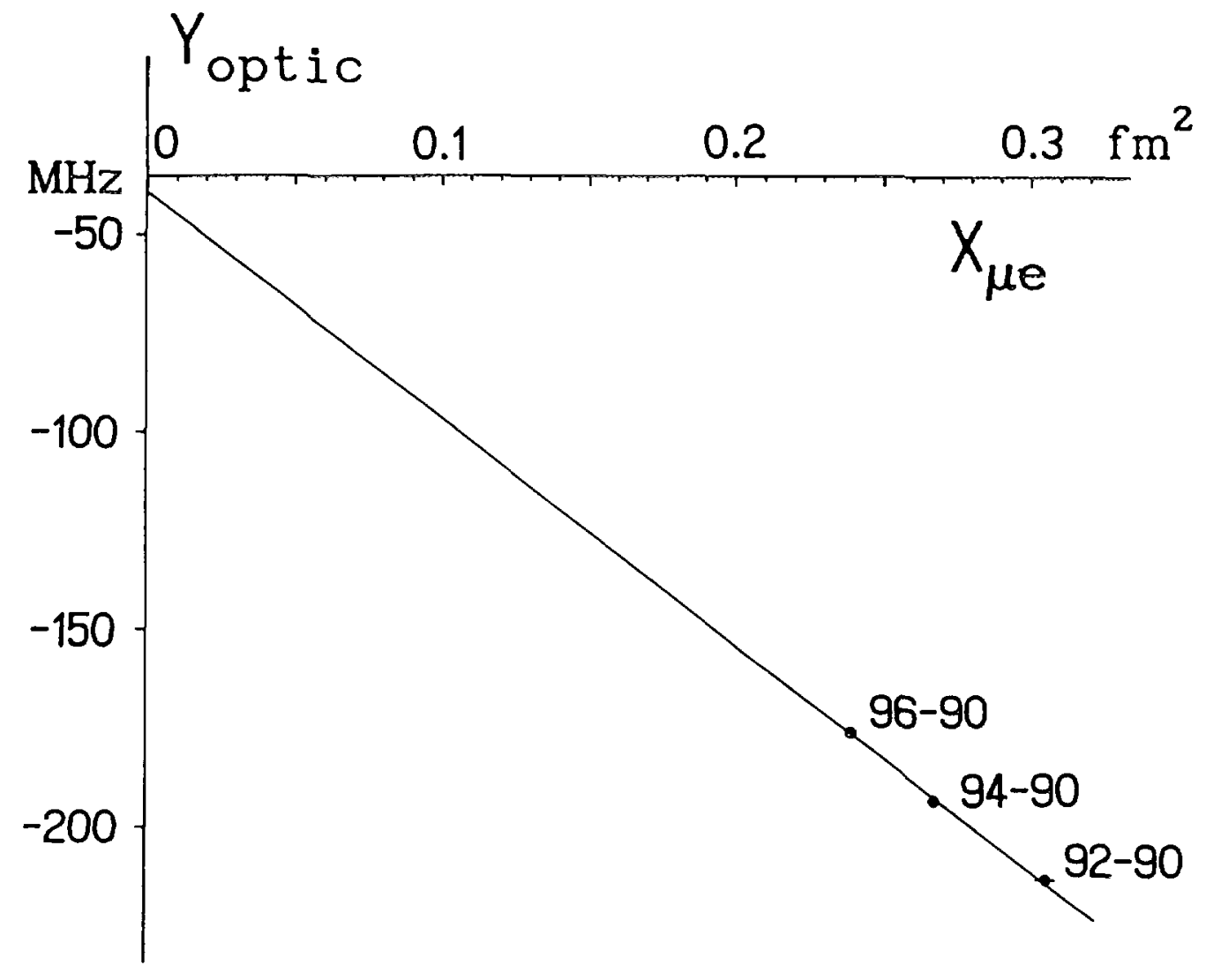

\begin{tabular}{|c|c|c|c|c|c|c|}
\hline Isotope & $v_{2}$ & $v_{4}$ & $v_{6}$ \\
\hline $90^{\circ}$ & 1.28071 & 1.1906 & 1.126 \\
$92^{e}$ & 1.28046 & 1.1899 & 1.125 \\
$94^{e}$ & 1.28031 & 1.1897 & 1.125 \\
96 & 1.28010 & 1.1891 & 1.124 \\
\hline
\end{tabular}

Note: The $V$ values for ${ }^{90,92,94} \mathrm{Zr}$ are taken from elastic electron scattering [Ma89]. The $V$ values for ${ }^{96} \mathrm{Zr}$ are extrapolated. 
TABLE X. King Plots: Optical versus Combined Muonic and Elastic Electron Scattering Data See page 194 for Explanation of Tables

\begin{tabular}{|c|c|c|c|c|}
\hline 42 MOLYBDENUM & $\lambda_{\text {proj }}$ & $=550.6 \mathrm{~nm}$ & $F_{550.6 \mathrm{~nm}}$ & $=-932(42) \frac{\mathrm{MHz}}{\mathrm{fm}^{2}}$ \\
\hline $\begin{array}{l}\mathrm{R}_{k \alpha}^{\mu}(96)=5.6125 \mathrm{fm} \\
C_{2} / C_{1}=-5.07 \cdot 10^{-4} \mathrm{fm}^{-2} \\
C_{3} / C_{1}=1.72 \cdot 10^{-6} \mathrm{fm}^{-4}\end{array}$ & $\begin{array}{l}\mathrm{FS}^{96-94} \\
\mathrm{MS}^{96-94} \\
\chi^{2} / \mathrm{D} \cdot \mathrm{F} .\end{array}$ & $\begin{array}{l}=-254(23) \mathrm{MHz} \\
=66(12) \mathrm{MHz} \\
=0.55\end{array}$ & $\begin{array}{l}\text { NMS }^{96-94} \\
\text { SMS }^{96-94} \\
\text { SMS/NMS }\end{array}$ & $\begin{array}{l}=66.3 \mathrm{MHz} \\
=-0.4(11.5) \mathrm{MHz} \\
=0.01(.17)\end{array}$ \\
\hline
\end{tabular}

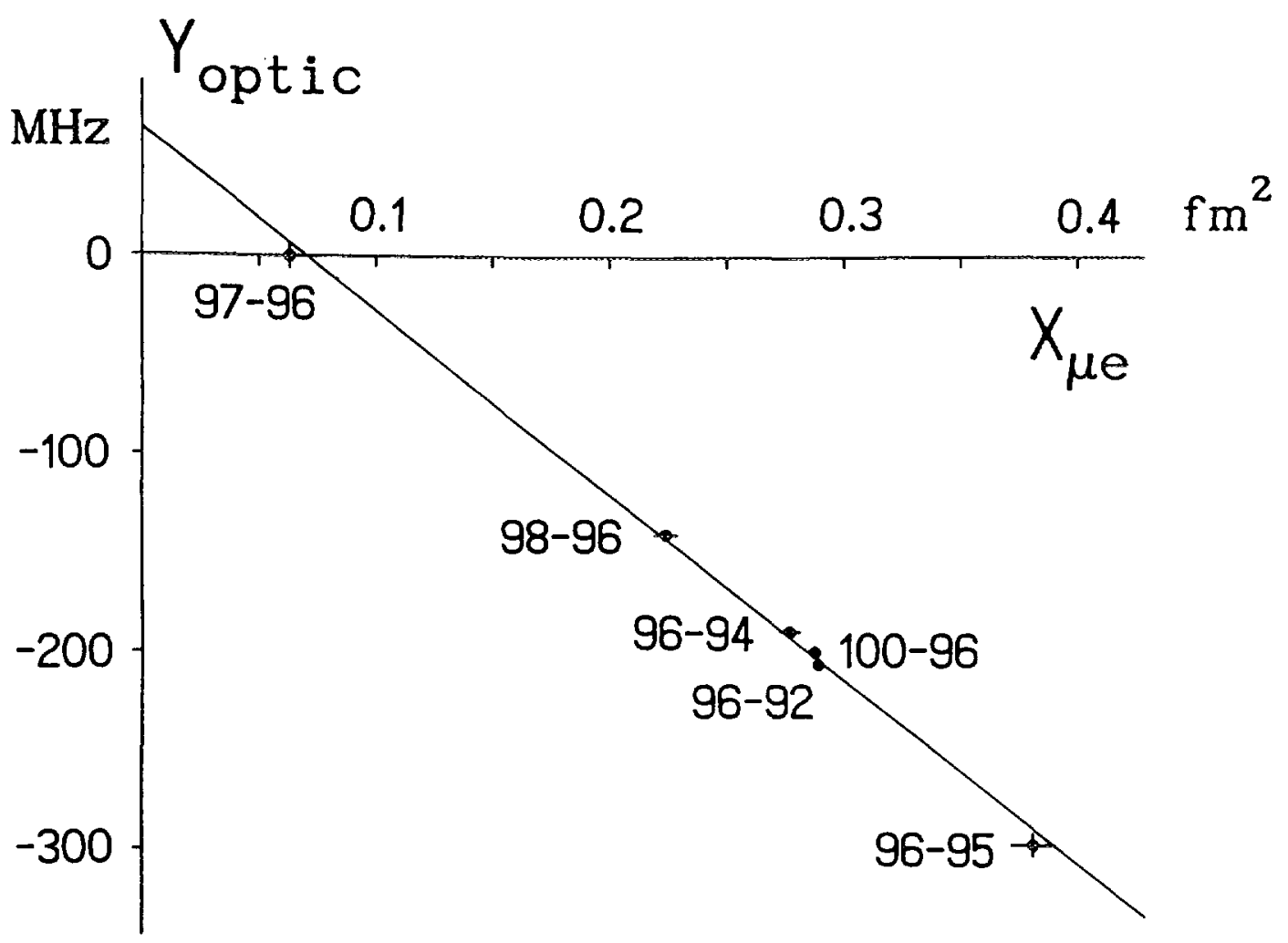

\begin{tabular}{|c|c|c|c|}
\hline Isotope & $v_{2}$ & $v_{4}$ & $v_{6}$ \\
\hline $92^{\circ}$ & 1.28088 & 1.1924 & 1.128 \\
94 & 1.28049 & 1.1912 & 1.126 \\
95 & 1.28039 & 1.1910 & 1.126 \\
$96^{\circ}$ & 1.28021 & 1.1906 & 1.126 \\
97 & 1.28010 & 1.1902 & 1.125 \\
$98^{\circ}$ & 1.27994 & 1.1898 & 1.125 \\
100 & 1.27971 & 1.1892 & 1.123 \\
\hline
\end{tabular}

\begin{tabular}{|c|l|c|}
\hline $\begin{array}{c}\text { Isotope } \\
\text { pair }\end{array}$ & $\begin{array}{l}\delta<\mathrm{r}^{2}>\text { ope } \\
{\left[\mathrm{fm}^{2}\right]}\end{array}$ & $\begin{array}{c}\mathrm{HM} \\
{[\%]}\end{array}$ \\
\hline $96-92$ & $0.609(28)$ & -2.34 \\
$96-94$ & $0.280(13)$ & -2.33 \\
$96-95$ & $0.197(9)$ & -2.34 \\
& & -2.57 \\
$97-96$ & $0.035(4)$ & -2.39 \\
$98-96$ & $0.218(10)$ & -2.37 \\
$100-96$ & $0.548(25)$ & \\
\hline
\end{tabular}

Note: The $V$ values ${ }^{92,96,98}$ Mo isotopes are taken from elastic electron scattering [Ma89]. The $V$ values for the other isotopes are inter-and extrapolated. 
TABLE X. King Plots: Optical versus Combined Muonic and Elastic Electron Scattering Data See page 194 for Explanation of Tables

62 SAMARIUM
$\mathrm{R}_{\mathrm{k \alpha}}^{\mu}(152)=6.4891 \mathrm{fm}$
$\mathrm{C}_{2} / \mathrm{C}_{1}=-8.04 \cdot 10^{-4} \mathrm{fm}^{-2}$
$\mathrm{C}_{3} / \mathrm{C}_{1}=2.29 \cdot 10^{-6} \mathrm{fm}^{-4}$

\begin{tabular}{|llll}
$\lambda_{\text {proj }}$ & $=570.68 \mathrm{~nm}$ & $F_{570.68 \mathrm{~nm}}$ & $=-3860(86) \frac{\mathrm{MHz}}{\mathrm{fm}^{2}}$ \\
$\mathrm{FS}^{152-150}$ & $=-1829(80) \mathrm{MHz}$ & $\mathrm{NMS}^{152-150}$ & $=25.3 \mathrm{MHz}$ \\
$\mathrm{MS}^{152-150}$ & $=35(32) \mathrm{MHz}$ & $\mathrm{SMS}^{152-150}$ & $=10(32) \mathrm{MHz}$ \\
$\chi^{2} / \mathrm{D} . \mathrm{F}$ & $=0.88$ & SMS $/ \mathrm{NMS}$ & $=0.4(1.3)$
\end{tabular}

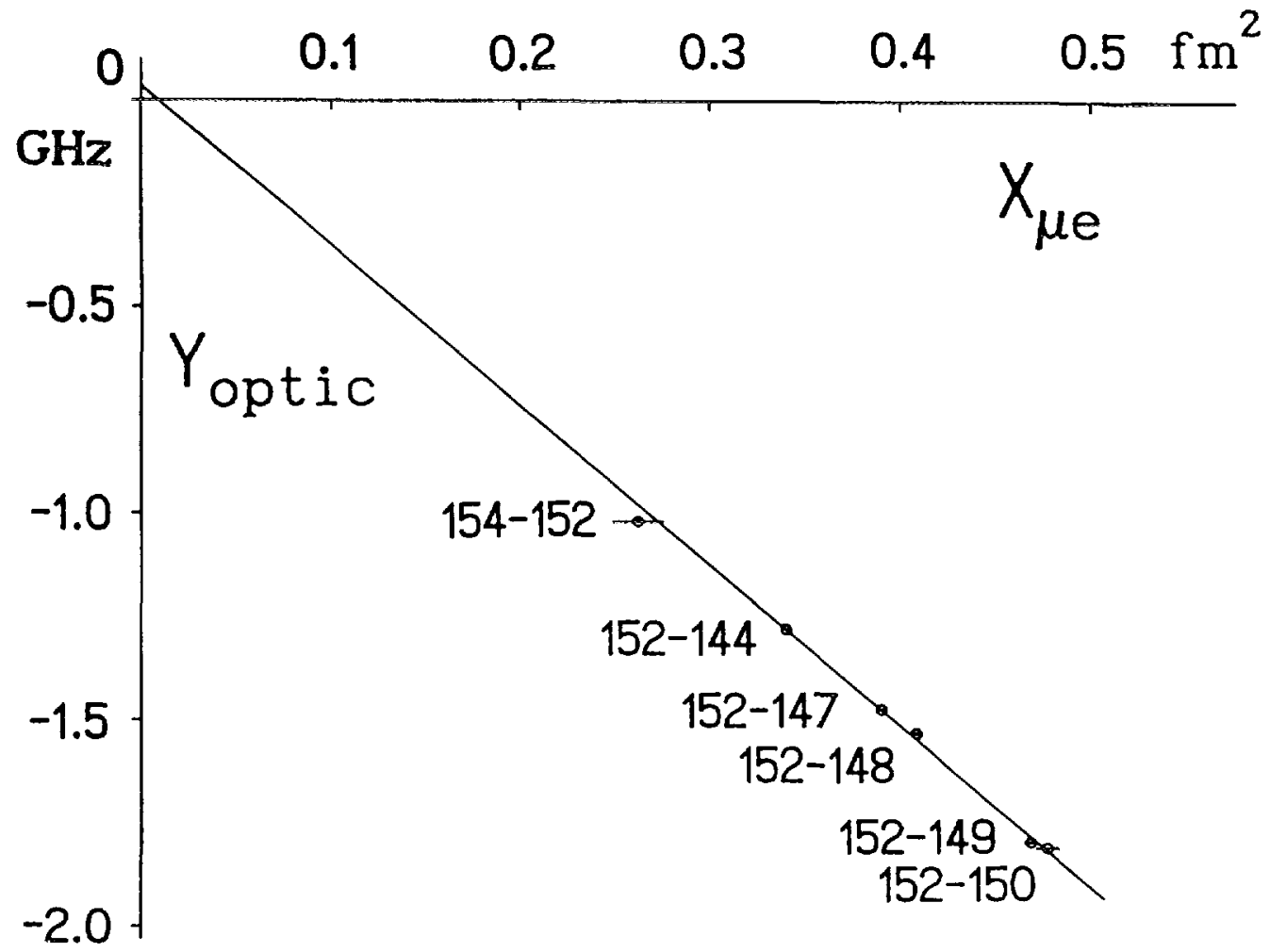

\begin{tabular}{|l|c|c|c|}
\hline Isotope & $v_{2}$ & $v_{4}$ & $v_{6}$ \\
\hline 144 & 1.28130 & 1.2049 & 1.152 \\
147 & 1.27950 & 1.1997 & 1.143 \\
$148^{\circ}$ & 1.27893 & 1.1982 & 1.141 \\
149 & 1.27820 & 1.1963 & 1.138 \\
150 & 1.27760 & 1.1946 & 1.135 \\
$152^{\circ}$ & 1.27594 & 1.1899 & 1.127 \\
$154^{\circ}$ & 1.27549 & 1.1888 & 1.125 \\
\hline
\end{tabular}

\begin{tabular}{|c|l|l|}
\hline $\begin{array}{c}\text { Isotope } \\
\text { pair }\end{array}$ & $\begin{array}{l}\delta<\mathrm{r}^{2}>\text { ope } \\
{\left[\mathrm{fm}^{2}\right]}\end{array}$ & $\begin{array}{c}\text { HM } \\
{[\%]}\end{array}$ \\
\hline $152-144$ & $1.497(34)$ & -5.65 \\
$152-147$ & $1.051(24)$ & -5.63 \\
$152-148$ & $0.868(20)$ & -5.68 \\
$152-149$ & $0.753(17)$ & -5.61 \\
$152-150$ & $0.503(11)$ & -5.73 \\
$154-152$ & $0.279(6)$ & -5.23 \\
\hline
\end{tabular}

Note: The $V$ values for ${ }^{148,152,154} \mathrm{Sm}$ are taken from elastic electron scattering [Vr87]. The $V$ values for the other isotopes are inter- and extrapolated. 
TABLE X. King Plots: Optical versus Combined Muonic and Elastic Electron Scattering Data See page 194 for Explanation of Tables

$\left.\begin{array}{|l}64 \text { GADOLINIUM } \\ \mathrm{R}_{\mathrm{k \alpha}}^{\mu}(156)=6.5617 \mathrm{fm} \\ \mathrm{C}_{2} / \mathrm{C}_{1}=-8.32 \cdot 10^{-4} \mathrm{fm}^{-2} \\ \mathrm{C}_{3} / \mathrm{C}_{1}=2.33 \cdot 10^{-6} \mathrm{fm}^{-4}\end{array}\right]\left[\begin{array}{llll}\lambda_{\text {proJ }} & =575.188 \mathrm{~nm} & F_{575.188} & =-7.22(.75) \frac{\mathrm{GHz}}{\mathrm{fm}^{2}} \\ \mathrm{FS}^{156-154} & =-1.50(.30) \mathrm{GHz} \mathrm{NMS}^{156-154} & =23.8 \mathrm{MHz} \\ \mathrm{MS}^{156-154} & =53(127) \mathrm{MHz} & \mathrm{SMS}^{156-154} & =29(127) \mathrm{MHz} \\ \chi^{2} / \mathrm{D} . \mathrm{F} . & =0.98 & \text { SMS/NMS } & =1.2(5.4) \\ \hline\end{array}\right.$

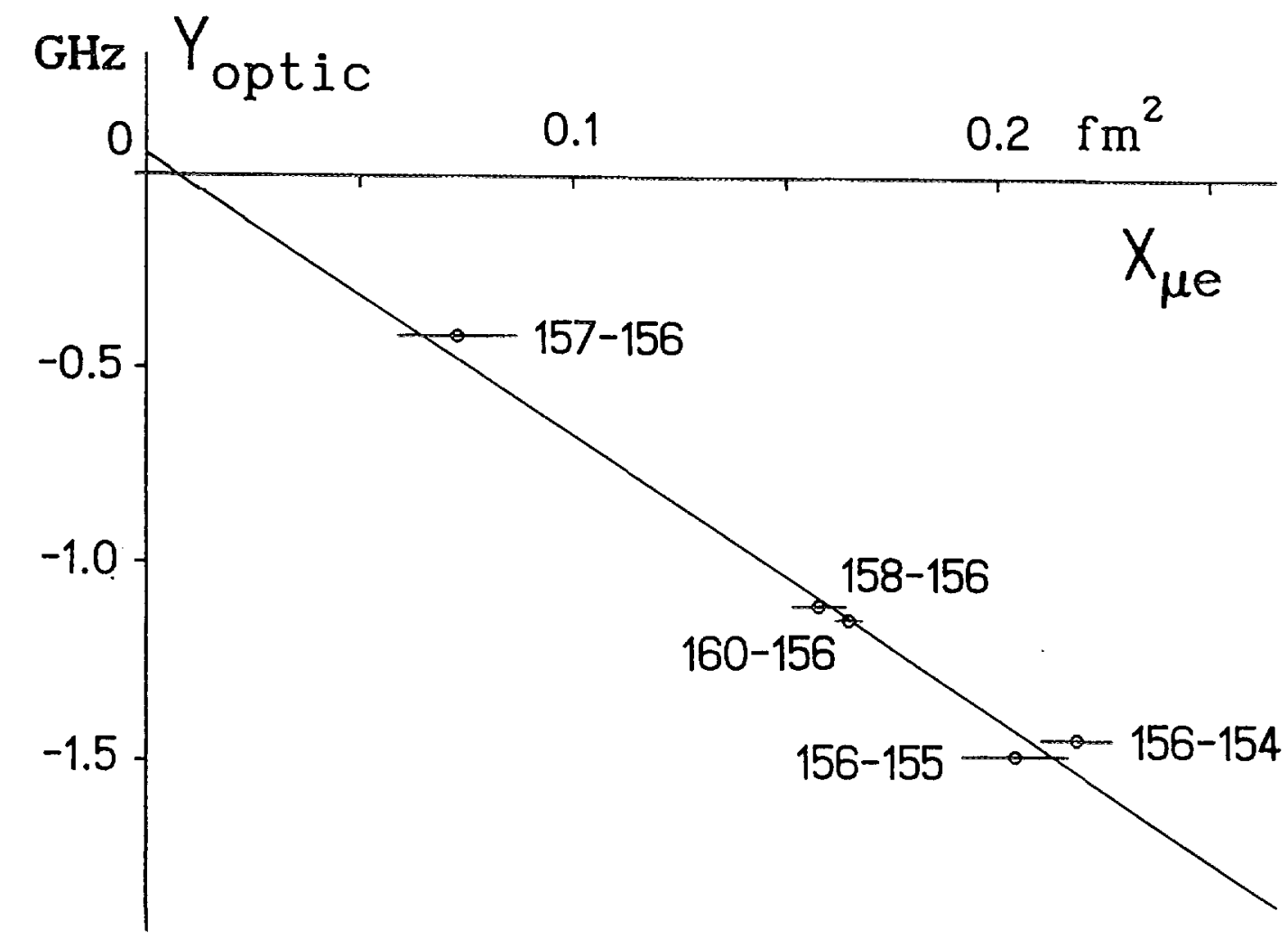

\begin{tabular}{|c|c|c|c|c|c|c|}
\hline Isotope & $V_{2}$ & $v_{4}$ & $V_{6}$ & $\begin{array}{c}\text { Isotope } \\
\text { pair }\end{array}$ & $\begin{array}{l}\delta<r^{2}>\text { ore } \\
{\left[\mathrm{fm}^{2}\right]}\end{array}$ & $\begin{array}{l}\mathrm{HM} \\
{[\%]}\end{array}$ \\
\hline $\begin{array}{l}154 \\
155 \\
156 \\
157 \\
158^{\circ} \\
160\end{array}$ & $\begin{array}{l}1.27513 \\
1.27513 \\
1.27513 \\
1.27513 \\
1.27513 \\
1.27513\end{array}$ & $\begin{array}{l}1.1884 \\
1.1884 \\
1.1884 \\
1.1884 \\
1.1884 \\
1.1884\end{array}$ & $\begin{array}{l}1.125 \\
1.125 \\
1.125 \\
1.125 \\
1.125 \\
1.125\end{array}$ & $\begin{array}{l}156-154 \\
156-155 \\
157-156 \\
158-156 \\
160-156\end{array}$ & $\begin{array}{l}0.218(23) \\
0.111(12) \\
0.034(4) \\
0.165(17) \\
0.335(35)\end{array}$ & $\begin{array}{l}-5.03 \\
-5.04 \\
-5.05 \\
-5.06 \\
-5.07\end{array}$ \\
\hline
\end{tabular}

Note: The $V$ values for ${ }^{158} \mathrm{Gd}$ are taken from elastic electron scattering [Vr87]. For the other isotopes the same $V$ values have been used. 
TABLE X. King Plots: Optical versus Combined Muonic and Elastic Electron Scattering Data See page 194 for Explanation of Tables

\begin{tabular}{|c|c|c|c|c|}
\hline 82 LEAD & $\lambda_{\text {proj }}$ & & $\mathrm{F}_{283.3 \mathrm{~nm}}$ & $=16.5(2.5) \frac{\mathrm{GHz}}{\mathrm{fm}^{2}}$ \\
\hline $\begin{array}{l}\mathrm{R}_{\mathrm{k} \alpha}^{\mu}(208)=7.031 \mathrm{fm} \\
\mathrm{C}_{2} / \mathrm{C}_{1}=-1.12 \cdot 10^{-3} \mathrm{fm}^{-2} \\
\mathrm{C}_{3} / \mathrm{C}_{1}=2.97 \cdot 10^{-6} \mathrm{fm}^{-4}\end{array}$ & $\begin{array}{l}\mathrm{FS}^{208-206} \\
\mathrm{MS}^{208-206} \\
\chi^{2} / \mathrm{D} . \mathrm{F}\end{array}$ & $\begin{array}{l}=1.90(.51) \mathrm{GHz} \\
=244(284) \mathrm{MHz} \\
=0.00\end{array}$ & $\begin{array}{l}\text { NMS }^{208-206} \\
\text { SMS } \\
\text { SMS/NMS }\end{array}$ & $\begin{array}{l}=27.1 \mathrm{MHz} \\
=217(284) \mathrm{MHz} \\
=8(10)\end{array}$ \\
\hline
\end{tabular}

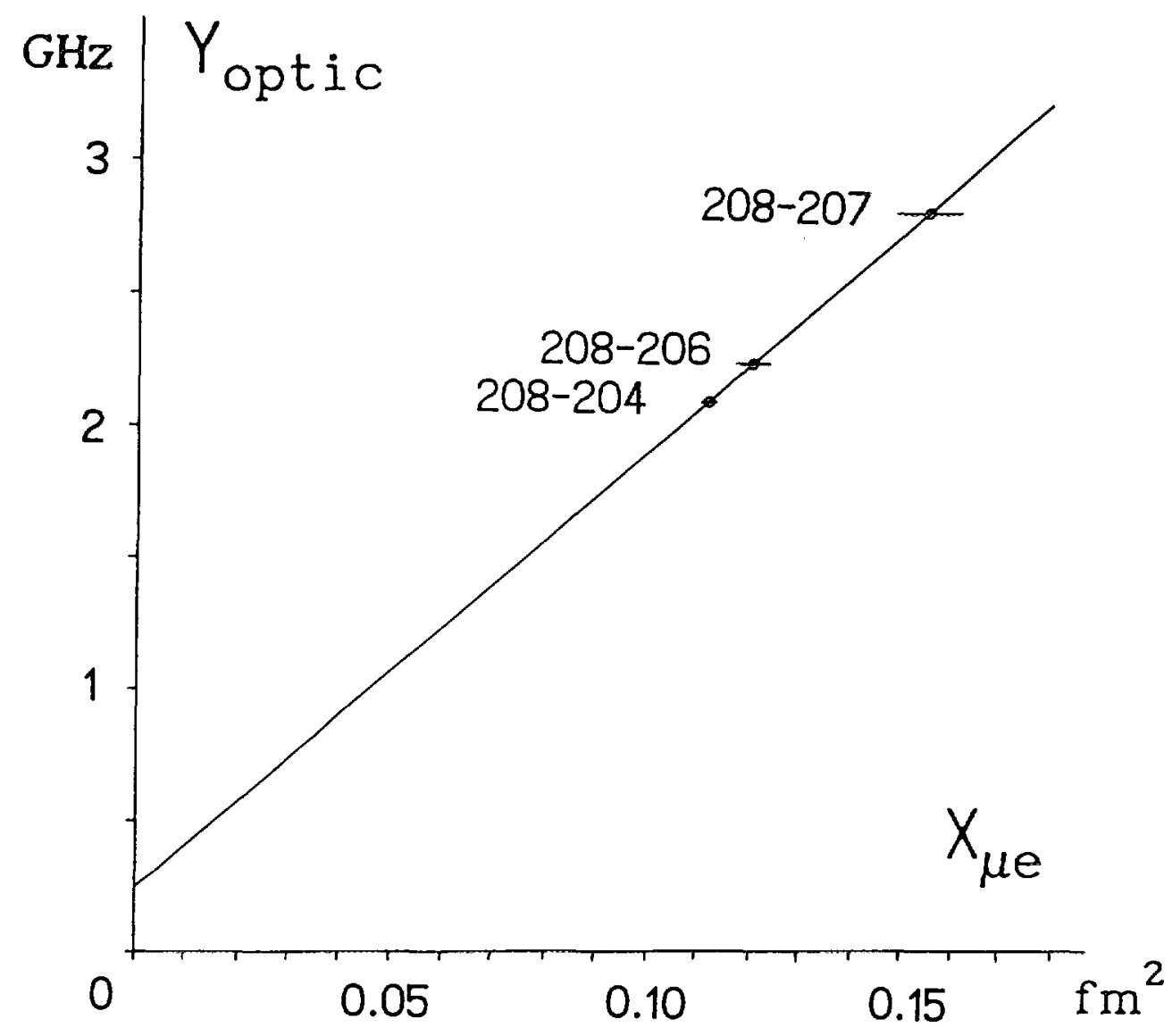

\begin{tabular}{|c|c|c|c|}
\hline Isotope & $v_{2}$ & $v_{4}$ & $v_{6}$ \\
\hline $204^{e}$ & 1.27808 & 1.2025 & 1.149 \\
$206^{\circ}$ & 1.27811 & 1.2027 & 1.150 \\
$207^{\circ}$ & 1.27809 & 1.2026 & 1.149 \\
$208^{e}$ & 1.27805 & 1.2026 & 1.149 \\
\hline
\end{tabular}

\begin{tabular}{|c|l|l|}
\hline $\begin{array}{c}\text { Isotope } \\
\text { palr }\end{array}$ & $\begin{array}{l}\left.\delta<\mathrm{r}^{2}\right\rangle_{\text {ope }} \\
{\left[\mathrm{fm}^{2}\right]}\end{array}$ & $\begin{array}{l}\mathrm{HM} \\
{[\%]}\end{array}$ \\
\hline $208-204$ & $0.240(39)$ & -7.55 \\
$208-206$ & $0.128(21)$ & -7.91 \\
$208-207$ & $0.080(13)$ & -7.89 \\
\hline
\end{tabular}

continued 
TABLE X. King Plots: Optical versus Combined Muonic and Elastic Electron Scattering Data See page 194 for Explanation of Tables

\begin{tabular}{|c|c|c|c|c|c|c|c|}
\hline Is.pair & $\delta\left\langle r^{2}\right\rangle_{\text {oнe }}$ & Is.pair & $\delta\left\langle r^{2}\right\rangle_{\text {ope }}$ & Is.pair & $\delta\left\langle r^{2}\right\rangle_{\text {ope }}$ & Is.pair & $\delta\left\langle r^{2}>\right.$ ope \\
\hline $208-196$ & $0.65(11)$ & $208-200$ & $0.461(75)$ & $208-205$ & $0.213(35)$ & $212-208$ & $0.455(74)$ \\
\hline $208-197$ & $0.65(11)$ & $208-201$ & $0.442(72)$ & $209-208$ & $0.103(17)$ & $214-208$ & $0.67(11)$ \\
\hline $208-198$ & $0.560(92)$ & $208-202$ & $0.353(58)$ & $210-208$ & $0.232(38)$ & & \\
\hline $208-199$ & $0.557(91)$ & $208-203$ & $0.330(54)$ & $211-208$ & $0.329(54)$ & & \\
\hline
\end{tabular}

Note: In lead the $V$ factors for all stable have been evaluated [Ma92a] from elastic electron scattering. Taking into account their errors $\left(\approx 10^{-4}\right)-$ which is in contrast to the policy of this Table $X$ - increases the limits of error of $F$. (see Table XII) by a factor of two, whereas the rms radil for lead stay un- ${ }^{1}$ changed. The situation for the other elements is quite different, for example in $\mathrm{Sm}$ (see Figs. 9 and 10).

The $V$ values for the unstable isotopes are the average of the stable ones, namely $1.2781,1.2026$, and 1.149 , respectively. 
TABLE XI. Electronic Factor $F$ and Specific to Normal Mass Shift Ratio SMS/NMS for Projected Optical Lines

See page 194 for Explanation of Tables

\begin{tabular}{|c|cl|c|ccc|}
\hline Element & $\begin{array}{c}\lambda \\
{[\mathrm{nm}]}\end{array}$ & $\begin{array}{l}\text { type of } \\
\text { transition }\end{array}$ & $\begin{array}{c}\text { SMS/NMS } \\
*\end{array}$ & $\begin{array}{c}\mathrm{F}_{1}\left[\mathrm{GHz} / \mathrm{fm}^{2}\right] \\
*)\end{array}$ & calc. (MCDF) & Ref. \\
\hline $\mathrm{Ca}$ & $2 \cdot 599.9$ & $4 \mathrm{~s}^{2} \rightarrow 4 \mathrm{~s} 5 \mathrm{~s}$ & $-0.0409(2)$ & $-0.0937(16)$ & $-0.077(1)$ & [To85] \\
$\mathrm{Kr}$ & 810.7 & $5 \mathrm{~s} \rightarrow 5 \mathrm{p}$ & $-0.08(6)$ & $-0.438(86)$ & & \\
$\mathrm{Sr}$ & 293.2 & $5 \mathrm{~s}^{2} \rightarrow 5 \mathrm{~s} 6 \mathrm{p}$ & $0.27(4)$ & $-0.611(81)$ & $-0.686(26)$ & [To85] \\
$\mathrm{Zr}$ & 613.46 & $5 \mathrm{~s}^{2} \rightarrow 5 \mathrm{~s} 5 \mathrm{p}$ & $-1.60(11)$ & $-0.566(28)$ & $-0.435(63)$ & [Fr93b] \\
$\mathrm{Mo}$ & 550.6 & $5 \mathrm{~s}^{2} \rightarrow 5 \mathrm{p}$ & $0.01(17)$ & $-0.932(42)$ & -0.93 & [Fr83] \\
$\mathrm{Sm}$ & 570.68 & $6 \mathrm{~s}^{2} \rightarrow 6 \mathrm{~s} 6 \mathrm{p}$ & $0.4(13)$ & $-3.860(86)$ & & \\
$\mathrm{Gd}$ & 575.19 & $6 \mathrm{~s}^{2} \rightarrow 6 \mathrm{~s} 6 \mathrm{p}$ & $1.2(54)$ & $-7.22(75)$ & & [Fr88b] \\
$\mathrm{Pb}$ & 283.3 & $6 \mathrm{p}^{2} \rightarrow 6 \mathrm{p} 7 \mathrm{~s}$ & $\mathbf{8 ( 1 0 )}$ & $16.5(25)$ & $20.6(3)$ & \\
\hline
\end{tabular}

*) experímental values, taken from Table X. 
TABLE XII. Root-Mean-Square Charge Radii from the Combined Analysis of Optical, Muonic, and Elastic Electron Scattering Data

See page 194 for Explanation of Tables

\begin{tabular}{|c|c|c|}
\hline Isotope & $\begin{array}{c}\left\langle r^{2}\right\rangle_{\text {ope }}^{1 / 2} \\
{[\mathrm{fm}]}\end{array}$ & $\begin{array}{c}\Delta\left\langle r^{2}\right\rangle^{1 / 2} \\
{[\mathrm{am}]}\end{array}$ \\
\hline${ }^{40} \mathrm{Ca}$ & $3.4767(8)$ & 0.0 \\
\hline${ }^{42} \mathrm{Ca}$ & $3.5057(9)$ & 0.4 \\
\hline${ }^{43} \mathrm{Ca}$ & $3.4928(8)$ & -0.9 \\
\hline${ }^{44} \mathrm{Ca}$ & $3.5152(9)$ & 0.0 \\
\hline${ }^{40} \mathrm{Ca}$ & $3.4921(9)$ & 2.5 \\
\hline${ }^{48} \mathrm{Ca}$ & $3.4736(8)$ & 0.2 \\
\hline${ }^{78} \mathrm{Kr}$ & $4.2032(12)$ & -0.1 \\
\hline${ }^{80} \mathrm{Kr}$ & $4.1976(9)$ & 0.0 \\
\hline${ }^{82} K r$ & $4.1921(11)$ & 0.2 \\
\hline${ }^{83} \mathrm{Kr}$ & $4.1860(14)$ & 0.0 \\
\hline${ }^{84} \mathrm{Kr}$ & $4.1884(12)$ & -0.1 \\
\hline${ }^{80} K r$ & $4.1839(13)$ & 0.0 \\
\hline${ }^{84} S r$ & $4.2365(13)$ & 0.0 \\
\hline${ }^{80} \mathrm{Sr}$ & $4.2261(9)$ & -0.1 \\
\hline${ }^{87} \mathrm{Sr}$ & $4.2190(8)$ & 0.1 \\
\hline${ }^{88} S r$ & $4.2188(8)$ & 0.0 \\
\hline${ }^{00} \mathrm{Zr}$ & $4.2692(10)$ & 0.0 \\
\hline${ }^{02} \mathrm{Zr}$ & $4.3055(10)$ & 0.1 \\
\hline${ }^{94} Z r$ & $4.3314(11)$ & 0.1 \\
\hline${ }^{06} Z r$ & $4.3508(12)$ & 0.0 \\
\hline${ }^{02} \mathrm{Mo}$ & $4.3146(11)$ & 0.1 \\
\hline${ }^{04} \mathrm{Mo}$ & $4.3518(10)$ & -0.1 \\
\hline${ }^{\mathrm{DS}} \mathrm{Mo}$ & $4.3617(9)$ & 0.3 \\
\hline${ }^{90} \mathrm{Mo}$ & $4.3840(7)$ & 0.0 \\
\hline${ }^{97} \mathrm{Mo}$ & $4.3880(7)$ & -0.4 \\
\hline${ }^{08} M o$ & $4.4089(10)$ & 0.1 \\
\hline${ }^{100} M O$ & $4.4465(14)$ & 0.1 \\
\hline
\end{tabular}

\begin{tabular}{|c|c|c|}
\hline Isotope & $\begin{array}{c}\left\langle r^{2}\right\rangle_{\text {oue }}^{1 / 2} \\
{[\mathrm{fm}]}\end{array}$ & $\begin{array}{c}\Delta\left\langle r^{2}\right\rangle^{1 / 2} \\
{[\mathrm{am}]}\end{array}$ \\
\hline${ }^{144} \mathrm{Sm}$ & $4.9373(10)$ & 0.0 \\
\hline${ }^{147} \mathrm{Sm}$ & $4.9824(9)$ & -0.1 \\
\hline${ }^{148} \mathrm{Sm}$ & $5.0002(8)$ & -0.2 \\
\hline${ }^{149} \mathrm{Sm}$ & $5.0129(8)$ & 0.1 \\
\hline${ }^{150} \mathrm{Sm}$ & $5.0379(9)$ & 0.7 \\
\hline${ }^{152} \mathrm{Sm}$ & $5.0870(8)$ & -0.4 \\
\hline${ }^{154} \mathrm{Sm}$ & $5.1143(9)$ & -1.4 \\
\hline${ }^{184} G d$ & $5.1240(14)$ & -0.4 \\
\hline${ }^{155} \mathrm{Gd}$ & $5.1353(11)$ & 0.2 \\
\hline${ }^{156} G d$ & $5.1460(9)$ & -0.1 \\
\hline${ }^{157} \mathrm{Gd}$ & $5.1492(9)$ & 0.4 \\
\hline${ }^{158} G d$ & 5.1618 & -0.2 \\
\hline${ }^{100} G d$ & $5.1782(16)$ & 0.0 \\
\hline${ }^{106} \mathrm{~Pb}$ & $5.442(10)$ & \\
\hline${ }^{197} \mathrm{~Pb}$ & $5.442(10)$ & \\
\hline${ }^{198} \mathrm{~Pb}$ & $5.450(8)$ & \\
\hline${ }^{100} \mathrm{~Pb}$ & $5.450(8)$ & \\
\hline${ }^{200} P b$ & $5.459(7)$ & \\
\hline${ }^{201} \mathrm{~Pb}$ & $5.461(7)$ & \\
\hline${ }^{202} \mathrm{~Pb}$ & $5.469(5)$ & \\
\hline${ }^{203} \mathrm{~Pb}$ & $5.471(5)$ & \\
\hline${ }^{204} \mathrm{~Pb}$ & $5.4793(8)$ & 0.0 \\
\hline${ }^{205} \mathrm{~Pb}$ & $5.482(3)$ & \\
\hline${ }^{206} \mathrm{~Pb}$ & $5.4896(7)$ & 0.0 \\
\hline${ }^{207} \mathrm{~Pb}$ & $5.4938(8)$ & 0.0 \\
\hline${ }^{208} \mathrm{~Pb}$ & $5.5013(7)$ & 0.0 \\
\hline${ }^{200} \mathrm{~Pb}$ & $5.511(2)$ & \\
\hline${ }^{210} \mathrm{~Pb}$ & $5.523(3)$ & \\
\hline${ }^{211} \mathrm{~Pb}$ & $5.533(5)$ & \\
\hline${ }^{212} \mathrm{~Pb}$ & $5.545(7)$ & \\
\hline${ }^{214} \mathrm{~Pb}$ & $5.565(10)$ & \\
\hline
\end{tabular}




\section{REFERENCES FOR TABLES}

[An82] A. Andl, K. Bekk, S. Göring, A. Hanser, G. Nowicki, H. Rebel, G. Schatz and R.C. Thompson Phys. Rev. C26 (1982) 2194

[An85] M. Anselment, S. Chongkum, S. Göring, A. Hanser, G. Meisel, H. Rebel and G. Schatz Ann. Rep. Nucl. Phys. of KfK 3969 (1985) 63

[An86] M. Anselment, W. Faubel, S. Göring, A. Hanser, G. Meisel and G. Schatz Nucl. Phys. A451 (1986) 471

[An87] M. Anselment, K. Bekk, S. Chongkum, S. Göring, A. Hanser, H. Hoeffgen, W. Kälber, G. Meisel and $\mathrm{H}$. Rebel

Z. Phys. A326 (1987) 493

[As84] A. Aspect, J. Bauche, A.L.A. Fonseca, P. Grangier and G. Roger J. Phys. B17 (1984) 1761

[As91] A. Aspect, J. Bauche, M. Godefroid, P. Grangier, J.E. Hansen and N. Vaeck J. Phys. B24 (1991) 4077

[Au78] P. Aufmuth, H.P. Clieves, K. Heilig, A. Steudel, D. Wendlandt and J. Bauche Z. Phys. A285 (1978) 357

[Au87] P. Aufmuth, K. Heilig and A. Steudel

At. Data and Nucl. Data Tables 37 (1987) 455

[Ba72] H. Backe, R. Engfer, U. Jahnke, E. Kankeleit, R.M. Pearce, C. Petitjean, L. Schellenberg, H. Schneuwly, W.U. Schröder, H.K. Walter and A. Zehnder Nucl. Phys. A189 (1972) 472

[Ba81] P. Barreau, L. Roussel and R.J. Powers Nucl. Phys. A384 (1981) 446

[Be80] E. Bergmann, P. Bopp, Ch. Dorsch, K. Kowalski and F. Träger Z. Physik A294 (1980) 319

[Be88] P. Bergem, G. Piller, A. Rüetschi, L.A. Schaller, L. Schellenberg and H. Schneuwly Phys. Rev. C37 (1988) 2821

[Be90] C. Bernhardt, Diploma thesis Institut für Kernphysik, KPH 7/90 Universität Mainz 1990

[Be92] C. Bernhardt, Ph.D. thesis Institut für Kernphysik, KPH 6/92 Universität Mainz 1992

[Be93] C. Bernhardt, G. Fricke, C. Piller, L.A. Schaller, L. Schellenberg and E.B. Shera private communication (Mainz/Fribourg/Los Alamos 1993)

[Bh69] S.K. Bhattacherjee, F. Boehm and P.L. Lee Phys. Rev. 188 (1969) 1919

[B158] J.E. Blaise

Ann. Phys. 3 (1958) 1019

[Bo83] G.L. Borchert, O.W.B. Schult, J. Speth, P.G. Hansen, B. Jonson, H.L. Ravn and J.B. McGrory Il Nuovo Cimento A73 (1983) 273

[Bo88] W. Böglin, P. Egelhof, I. Sick, J.M. Cavedon, B. Frois, D. Goutte, V. Mwot, P. Leconte, X.H. Phan, S.K. Platchkov, S. Williamson and M. Girod Nucl. Phys. A477 (1988) 399 


\section{REFERENCES FOR TABLES continued}

[Br65] R.T. Brockmeier, F. Boehm and E.N. Hatch Phys. Rev. Lett. 15 (1965) 132

[Br79] H.W. Brandt, E. Meissner, and A. Steudel Z. Phys. A291 (1979) 97

[Br80] H. Brand, B. Seibert, and A. Steudel

Z. Phys. A296 (1980) 281

[Br84] T. Brenner, S. Büttgenbach, N. Glaeser, M. Koster, H. Roeder, W. Rupprecht and F. Träber Z. Phys. A316 (1984) 247

[Bu85] F. Buchinger, R. Corriveau, E.B. Ramsay, D. Berdichevsky and D.W.L. Sprung Phys. Rev. C32 (1985) 2058

[Bu88] P. Buch, J. Nellessen, and W. Ertmer Phys. Scr. 38 (1988) 664

[Bu89] A.J.C. Burghardt, Ph.D. thesis University of Amsterdam 1989

[Ca90] B.D. Cannon and G.R. Janik Phys. Rev. A42 (1990) 397

[Ch68] R.B. Chesler and F. Boehm Phys. Rev. 166 (1968) 1202

[Du90] S.B. Dutta, A.G. Martin, W.F. Rogers, and D.L. Clark Phys. Rev. C42 (1990) 1911

[Ei70a] C.W.E. Van Eijk and M.J.C. Visscher Phys. Lett. 34B (1970) 349

[Ei70b] C.W.E. Van Eijk and F. Schutte Nucl. Phys. A151 (1970) 459

[El83] E.R. Eliel, W. Hogervorst, T. Olsson and L.R. Pendrill Z. Physik A311 (1983) 1

[Em83] H.J. Emrich, Ph.D. thesis Institut für Kernphysik, Universität Mainz 1983

[En74] R. Engfer, H. Schneuwly, J.L. Vuilleumier, H.K. Walter and A. Zehnder At. Data and Nucl. Data Tables 14 (1974) 509

[En90] J.G. England, I.S. Grant, J.A.R. Griffith, D.E. Evans, D.A. Eastman, G.W.A. Newton and P.M. Walker J. Phys. G16 (1990) 105

[Fr82] G. Fricke, G. Mallot, H.G. Sieberling, T.Q. Phan, G. Piller, A. Rüetschi, L.A. Schaller, L. Schellenberg and H. Schneuwly SIN Newsletter 14 (1982) 61

[Fr83] B. Fricke and G. Torbohm private communication, Universität Kassel 1983

[Fr87] G. Fricke, T. Hack, T. Hennemann and E.B. Shera private communication (Mainz/Los Alamos 1987)

[Fr88a] G. Fricke, T. Hack, T. Hennemann, J. Herberz, G. Mallot, L.A. Schaller and L. Schellenberg private communication (Mainz/Fribourg 1988) 


\section{REFERENCES FOR TABLES continued}

[Fr88b] B. Fricke

private communication, Universität Kassel 1988

[Fr92] G. Fricke, J. Herberz, T. Hennemann, G. Mallot, L.A. Schaller, L. Schellenberg, C. Piller and R. Jacot-Guillarmod

Phys. Rev. C45 (1992) 80

[Fr93a] G. Fricke, C. Bernhardt, T. Hennemann, J. Herberz, G. Mallot, L.A. Schaller, L. Schellenberg and E.B. Shera

Confit, Institutsreport Kernphysik, KPH 16/93 Universität Mainz 1993

[Fr93b] B. Fricke

private communication, Universität Kassel 1993

[Gu83] C. Günther, E.B. Shera, M.V. Hoehn, H.D. Wohlfahrt, R.J. Powers, Y. Tanaka and

A.R. Kunselman

Phys. Rev. C27 (1983) 816

[Ha67] J.E. Hansen, A. Steudel and H. Walther

Z. Phys. 203 (1967) 296

[Ha79] A.A. Hahn, J.P. Miller, R.J. Powers, A. Zehnder, A.M. Rushton, R.E. Welsh, A.R. Kunselman, P. Roberson and H.K. Walter

Nucl. Phys. A314 (1979) 361

[Ha88] P.A. Hackett, H.D Morrison, O.L. Bourne, B. Simard, and D.M. Rayner

S. Opt. Soc. Am. B5 (1988) 2409

[Ha89] Th. Hack, Diploma thesis

Institut für Kernphysik, KPH 10/89 Universität Mainz 1989

[Ha92] P. Hannaford

Opt. Lett. 17 (1992) 432

[He84] T. Hennemann, Diploma thesis Institut für Kernphysik, KPH 2/84 Universität Mainz 1984

[He85] Th. Hennemann, G. Fricke, G. Mallot, P. Bergem, F. Bienz, N. Boschung, G. Piller, L.A. Schaller, L. Schellenberg and H. Schneuwly

SIN Newsletter 17 (1985) 31

[He86] Th. Hennemann and J. Herberz

Data [Ma83] reanalysed (Universität Mainz 1986)

[Ho81] M.V. Hoehn, E.B. Shera, H.D. Wohlfahrt, Y. Yamazaki, R.M. Steffen and R.K. Sheline Phys. Rev. C24 (1981) 1667

[Ho84] B. Hoffmann, G. Baur and J. Speth

Z. Phys. A315 (1984) 57

[Ja89] J. Jansen, Diploma thesis

Institut für Kernphysik, KPH 9/89 Universität Mainz 1989

[Ji90] W.G. Jin, H. Sakata, M. Wakasugi, T. Horiguchi and Y.Yoshizawa

Phys. Rev. A42 (1990) 1416

[Ke75] D. Kessler, H. Mes, A.C. Thompson, H.L. Anderson, M.S. Dixit, C.K. Hargrove and R.J. McKee Phys. Rev. C11 (1975) 1719 


\section{REFERENCES FOR TABLES continued}

[Kh88] A.A. Khomich, N.G. Shevchenko, E.O. Babichev, A.Yu. Buki, V.I. Polishchuk, B.V. Mazanko and V.P. Sergienko

Sov. J. Nucl. Phys. 47 (1988) 191

[Kh90] A.A. Khomich, N.G. Shevchenko, A.Yu. Buki, B.V. Mazan'ko, V.I. Polishchuk and Yu.N. Ranyuk Sov. J. Nucl. Phys. 51 (1990) 17

[K188] R.M. Klein, Diploma thesis Institut für Kernphysik, KPH 8/88 Universität Mainz 1988

[Ko81] J. Konijn, W. van Doesburg, G.T. Ewan, T. Johansson and G. Tibell Nucl. Phys. A360 (1981) 187

[Kr85] J.R. Kropp, H.D. Kronfeldt, and R. Winkler

Z. Phys. A321 (1985) 57

[Kr90] H.D. Kronfeldt, G. Klemz, and D.J. Weber

J. Phys. B23 (1990) 1107

[Ku83] W. Kunold, M. Schneider, L.M. Simons, J. Wüest and R. Abela

Z. Phys. A313 (1983) 11

[La83] D.B. Laubacher, Y. Tanaka, R.M. Steffen, E.B. Shera and M.V. Hoehn Phys. Rev. C27 (1983) 1772

[La86] J. Laksanaboonsong, Ph.D. thesis University of Virginia (1986)

[La93] E. Langlois and J.-M. Gagnee

J. Opt. Soc Am. B10 (1993) 774

[Le73] P.L. Lee and F. Boehm

Phys. Rev. 8 (1973) 819

[Lo83] C.-J. Lorenzen, K. Niemax and L.R. Pendrill

Phys. Rev. 28 (1983) 2051

[Ma83] G. Mallot, G. Fricke, L.A. Schaller and L. Schellenberg private communication (Mainz/Fribourg 1983)

[Ma85] G. Mallot, Ph.D. thesis Institut für Kernphysik, KPH 1/85 Universität Mainz 1985

[Ma89] P. Mazanek, Diploma thesis Institut für Kernphysik, KPH 11/89 Universität Mainz 1989

[Ma92a] P. Mazanek, Ph.D. thesis Institut für Kernphysik, KPH 5/92 Universität Mainz 1992

[Ma92b] A.M. Mårtensson-Pendrill, A. Yunarmann, H. Warston, L. Vermeeren, R.E Silverans, A. Klein, R. Neugart, Ch. Schulz, P. Lievens and the ISOLDE - Collaboration

Phys. Rev. A45 (1992) 4675

[Of91] E.A.J.M. Offermann, L.S. Cardman, C.W. de Jager, H. Miska, C. de Vries and H. de Vries Phys. Rev. C44 (1991) 1096

[Ol86] T. Olsson, L. Fraenkel, L. Lindgren, A. Nyberg, L. Robertsson and A. Rosén Physica Scr. 34 (1986) 24 


\section{REFERENCES FOR TABLES continued}

[Pa84] C.W.P. Palmer, P.E.G. Baird, S.A. Blundell, J.R. Brandenberger, C.J. Foot, D.N. Stacey and G.K. Woodgate J. Phys. B17 (1984) 2197

[Ph85] T.Q. Phan, P. Bergem, A. Rüetschi, L.A. Schaller and L. Schellenberg Phys. Rev. C32 (1985) 609

[Pi90] C. Piller, C. Gugler, R. Jacot-Guillarmod, L.A. Schaller, L. Schellenberg, H. Schneuwly, G. Fricke, Th. Hennemann and J. Herberz

Phys. Rev. C42 (1990) 182

[Po68] R.J. Powers

Phys. Rev. 169 (1968) 1

[Po76] R.J. Powers, F. Boehm, P. Vogel, A. Zehnder, T. King, A.R. Kunselmann, P. Roberson, P. Martin, G.H. Miller, R.E Welsh and D.A. Jenkins

Nucl. Phys. A262 (1976) 493

[Po77] R.J. Powers, F. Boehm, A. Zehnder, A.R. Kunselman and P. Roberson

Nucl. Phys. A278 (1977) 477

[Po79] R.J. Powers, P. Barreau, B. Bihoreau, J. Miller, J. Morgenstern, J. Picard and L. Roussel Nucl. Phys. A316 (1979) 295

[Re87] M. Reutter, Diploma thesis Institut für Kernphysik, KPH 6/87 Universität Mainz 1987

[Ri76] G.A. Rinker

L.A.N.L., Los Alamos, NM, private communication 1976

[Ri78] G.A. Rinker and J. Speth

Nucl. Phys. A306 (1978) 397

[Ru84a] W. Ruckstubl, B. Aas, W. Beer, I. Beltrami, K. Bos, P.F.A. Goudsmit, H.J. Lejsi, G. Strassner, A. Vacchi, F.W.N. de Boer, U. Kiebele and R. Weber

Nucl. Phys. A430 (1984) 685

[Ru84b] A. Rüetschi, L. Schellenberg, T.Q. Phan, G. Piller, L.A. Schaller, and H. Schneuwly Nucl. Phys. A422 (1984) 461

[Ry72] A.S. Rylnikov, A.I. Egorov, G.A. Ivanov, V.I. Marushenko, A.F. Mezentsev A.I. Smirnov, O.I. Sumbaev and V.V. Fyodorov

Zh. Eksp. Teor. Fiz. 63 (1972) 53

[Sc80a] L.A. Schaller, L. Schellenberg, A. Ruetschi and H. Schneuwly

Nucl. Phys. A343 (1980) 333

[Sc80b] L. Schellenberg, B. Robert-Tissot, K. Käser, L.A. Schaller, H. Schneuwly, G. Fricke, S. Glückert, G. Mallot and E.B. Shera

Nucl. Phys. A333 (1980) 333

[Sc82] L.A. Schaller, L. Schellenberg, T.Q. Phan, G. Piller, A. Rüetschi and H. Schneuwly Nucl. Phys. A379 (1982) 523

[Sc85] L.A. Schaller, D.A. Barandao, P. Bergem, M. Boschung, T.Q. Phan, G. Piller, A. Rüetschi, L. Schellenberg, H. Schneuwly, G. Fricke, G. Mallot and H.G. Sieberling

Phys. Rev. C31 (1985) 1007 


\section{REFERENCES FOR TABLES continued}

[Se69] E.C. Seltzer

Phys. Rev. 188 (1969) 1916

[Sh76] E.B. Shera, E.T. Ritter, R.B. Perkins, G.A. Rinker, L.K. Wagner, H.D. Wohlfahrt, G. Fricke and R.M. Steffen

Phys. Rev. C14 (1976) 731

[Sh82] E.B. Shera, H.D. Wohlfahrt, M.V. Hoehn and Y. Tanaka

Phys. Lett. B112 (1982) 124

[Sh89] E.B. Shera, M.V. Hoehn, G. Fricke and G. Mallot

Phys. Rev. C39 (1989) 195

[Si82a] H.G. Sieberling, Diploma thesis

Institut für Kernphysik, KPH 16/82 Universität Mainz 1982

[Si82b] H.G. Sieberling, G. Fricke, L.A. Schaller and L. Schellenberg private communication (Mainz/Fribourg 1982)

[So88] R. Soundranayagam, A. Saha, K.K. Seth, C.W. de Jager, H. de Vries, H. Blok and G. van der Steenhoven

Phys. Lett. B212 (1988) 13

[St52] A. Steudel

Z. Phys. 133 (1952) 438

[Su67] O.I. Sumbaev, Symposium on Nuclear Structure, Dubna 1968

IAEA, Vienna, 1968, p.527

O.I. Sumbaev, E.V. Petrovich, V.S. Sykor, A.S. Rylnikov and A.I. Grushko

Sov. J. Nucl. Phys. 5 (1967) 387

[Su69] O.I. Sumbaev, A.F. Mezentsev, V.I. Marushenko, A.S. Rylnikov, G.A. Ivanov and A.I. Egorov Int. Conf. Prop. Nucl. States, Montreal, Canada, 1969 (Contrib. 2.67)

[Ta84a] Y. Tanaka, R.M. Steffen, E.B. Shera, W. Reuter, M.V. Hoehn and J.D. Zumbro Phys. Rev. C29 (1984) 1830

[Ta84b] Y. Tanaka, R.M. Steffen, E.B. Shera, W. Reuter, M.V. Hoehn and J.D. Zumbro Phys. Rev. C29 (1984) 1897

[Ta84c] Y. Tanaka, R.M. Steffen, E.B. Shera, W. Reuter, M.V. Hoehn and J.D. Zumbro Phys. Rev. C30 (1984) 350

[Th83] R.C. Thompson, M. Anselment, K. Bekk, S. Göring, A. Hanser, G. Meisel, H. Rebel, G. Schatz and B.A. Brown

J. Phys. G9 (1983) 443

[To85] G. Torbohm, B. Fricke and A. Rosén

Phys. Rev. A31 (1985) 2038

[Vr87] H. de Vries, C.W. de Jager and C. de Vries ATOMIC DATA AND NUCLEAR DATA TABLES 36 (1987) 495

[Vr88] J.W. de Vries, D. Doornhof, C.W. de Jager, R.P. Singhal, S. Salem, G.A. Peterson and R.S. Hicks

Phys. Lett. B205 (1988) 22 


\section{REFERENCES FOR TABLES continued}

[Wa90] M. Wakasugi, T. Horiguchi, W.G. Jin, H. Sakata and Y. Yoshizawa J. Phys. Soc. Japan 59 (1990) 2700

[We93] J. Wesseling, C.W. de Jager, L. Lapikas, H. de Vries, M.N. Harakeh, N. Kalantar-Nayestanaki, L.W. Fagg, R.A. Lindgren, E. Moya De Guerra and P. Sarriguren to be published

[Wo81] H.D. Wohlfahrt, E.B. Shera, M.V. Hoehn, Y. Yamazaki and R.M. Steffen Phys. Rev. C23 (1981) 533

[Ya78] Y. Yamazaki, E.B. Shera, M.V. Hoehn and R.M. Steffen Phys. Rev. C18 (1978) 1474

[Ze75] A. Zehnder, F. Boehm, W. Dey, R. Engfer, H.K. Walter and J.L. Vuilleumier Nucl. Phys. A254 (1975) 315

[Zu84] J.D. Zumbro, R.A. Naumann, M.V. Hoehn, W. Reuter, E.B. Shera, C.E. Bemis Jr. and Y. Tanaka

Phys. Rev. Lett. 20 (1984) 1888

[Zu86] J.D. Zumbro, R.A. Naumann, M.V. Hoehn, W. Reuter, E.B. Shera, C.E. Bemis Jr. and Y. Tanaka

Phys. Lett. 167B (1986) 383 